\title{
Estimación del tamaño \\ de los artefactos producidos en la elicitación de requerimientos
}

María Elena Centeno

Director: Lic. Alejandro Oliveros

Tesis presentada a la Facultad de Informática de la Universidad Nacional de La Plata como parte de los requisitos para la obtención del título de Magister en Ingeniería de Software.

Facultad de Informática Unive rsidad Nacional de La Plata, Argentina

2004 
La elaboración de esta tesis me ha permitido investigar áreas delconocimiento que no me eran familiares y no hubiera podido alcanzar plenamente mis objetivos sin la colaboración de otros profesionales que cooperaron desinteresadamente conmigo.

Para los temas relacionados con el Lenguaje Naturaly las Clases de Palabras que en definitiva me permitieron llegar al conce pto de Palabra Normalizada, concepto central de esta tesis, conté con la inestimable colaboración del Lic. Eduardo G. Bibiloni, Profesor de las cátedras de Gramática Española I, Gramática Española II y Lingǘstica II de la Facultad de Humanidades de la Universidad Nacional de la Patagonia.

O tro punto especialmente importante de este trabajo está relacionado con las mediciones y especificamente con la Estadística. En este tema conté con los aportes de la Lic. en Estadística Mirta Mas, especializada en docencia e investigación en Ciencias Económicas a cargo de la cátedra de Estadística en la Fac. de Cs. Económicas en la Sede Comodoro Rivadavia de la Universidad Nacional de la Patagonia.

$\mathcal{A}$ ellos vaya mi sincero reconocimiento y agradecimiento. 
1 Objetivo

2 Motivación

3 Metodología de trabajo

4 Propuestas relacionadas

5 Organización

II Elicitación de requerimientos 6

1 Ingeniería de Requerimientos

2 Elicitación de Requerimientos

3 Medición de Requerimientos

4 Escenarios

5 Léxico Extendido del Lenguaje

6 Aplicación al caso de estudio

II Teoríade mediciones

1 Mediciones en la Ingeniería del $S$ oftware

2 Definición de $\mathcal{M}$ edición

3 Teoría de las mediciones

4 Teoría Representacional de la Medición

5 Mediciones Inmediatas y Mediciones Calculadas

6 Proceso de medición

1 Definición de palabra normalizada

2 Técnica propues ta para aplicar las reglas de normalización

3 Reglas para definir una palabra normalizada

1 Mediciones inmediatas

1.1 Mediciones aplicadas a palabras normalizadas

2 Mediciones calculadas

3 Aplicación de las reglas de normalización y mediciones propuestas

4 Comentarios sobre Fan In y Fan Out

5 Descripción de la Tabla 1 del Apéndice $\mathcal{F}$ 
2 Mediciones calculadas

3 Descripción de la Tabla 2 del Apéndice $\mathcal{F}$

VIII Caso de estudio 64

1 Mediciones aplicadas al simbolo

1.1 Mediciones inmediatas

1.1.1 Cantidad de palabras

1.1.2 Cantidad de oraciones

1.1.3 Mediciones aplicadas a las palabras normalizadas

1.1.3.1 Cantidad de palabras normalizadas

1.1.3.2 Cantidad de verbos normalizados

1.1.3.3 Cantidad de simbolos o Fan In

1.1.3.4 Cantidad de otras palabras normalizadas

1.2 Mediciones calculadas

1.2.1 Coeficiente de redundancia

1.2.2 Coeficiente de simplicidad

1.2 .3 Fan Out

$1.2 .4 \mathcal{F O} I$

1.2.5 Tasa de uso

2 Mediciones aplicadas a las palabras del LEL

2.1 Mediciones inme diatas

2.1.1 Valores totales

2.1.2 Distribución de los simbolos según las clasificación

2.2 Mediciones calculadas

2.2.1 Mediciones de tendencia central

2.2.2 Mediciones de dispersión

2.2.3 Análisis de correlación

3 Resumen de las mediciones

$I X$

Anális is comparativo de casos

1 Anális is de las mediciones obtenidas

1.1 LEL "Hotel"

1.2 LEL "Pasaporte"

1.3 LEL "LEL \& E Ecenarios"

2 Comparación de los tres casos de estudio

3 Valores esperados para las mediciones

$x$

Conclusiones 100

1 Conclusiones

2 Pautas para construir un LEL me dible

3 Trabajos futuros

Bibliografía 104 
Apéndices

A Clases de palabras

$\mathcal{B} \quad$ Reglas de Normalización

C Definiciones de Mediciones Propuestas

$\mathcal{D} \quad$ Estadística

E LEL "Hotel"

$\mathcal{F}$ Tablas LEL "Hotel"

1 Mediciones aplicadas al simbolo

$2 \quad$ Mediciones aplicadas al $\mathcal{L E L}$

3 Matriz de adyacencia ponderada

G Tablas LEL "Pasaporte"

1 Mediciones aplicadas al simbolo

$2 \quad$ Mediciones aplicadas al $\mathcal{L E L}$

3 Matriz de adyacencia ponderada

$\mathcal{H} \quad \mathcal{T} a b l a s$ LEL "LEL y Escenarios"

1 Mediciones aplicadas al simbolo

$2 \quad$ Mediciones aplicadas al $\mathcal{L E L}$

3 Matriz de adyacencia ponderada 
Figura Descripción

Página

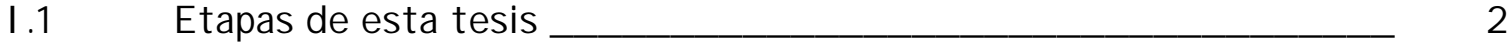

II.1 Ulbicación de los escenarios dentro de la $\mathcal{S E} \mathcal{E}_{---}$

II.2 Pasos para la construcción del LEL [Hadad 97$]$

II.3 Distribución según la clasificación particular_-__-_-_-_-_-_-_-_ 16

II.4 Distribución según la clasificacióngeneral_-__-_-_-_-_-_-__-_- 17

III.1 Condición de representación [Fenton 96]__-_______-__-__-_- 29

III.2 Relación entre los modelos y sus resultados [Offen 97]_-_-_-_-_- 30

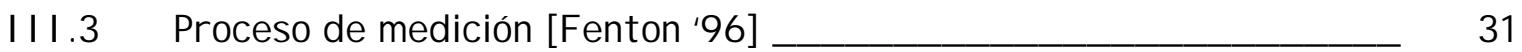

VI.1 Tabla 1 Apéndice F: Mediciones aplicadas al simbolo__-__-__-_-_ 57

VI.2 Fórmulas y funciones de la planilla de cálculo utilizadas__-_-_-_-_ 57

VII.1 Tabla 2 del Apéndice F: Mediciones estadísticas__-_-_-_-_-_-_- 62

VII.2 Tabla 2 del Apéndice F: Mediciones para la Población_-_-_-_-_-_- 63

VII.3 Tabla 2 del Apéndice F: Funciones de la planilla de cálculo_-_-_-_- 63

VIII.1 Distribución de la CPy CPN de las entradas del LEL__-_-_-_-_-_- 66

VIII.2 Distribución de la CPN de las entradas del LEL__-_-_-_-_-_-_-- 67

VIII.3 Composición porcentual de la CPN

VIII.4 CVN de las entradas del $\mathcal{L E} \mathcal{L}_{-}$

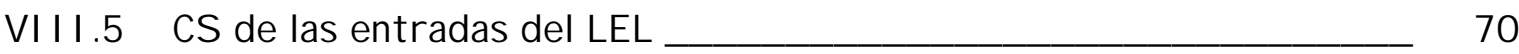

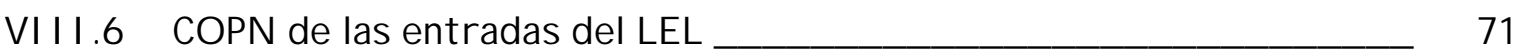

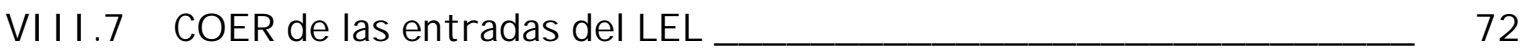

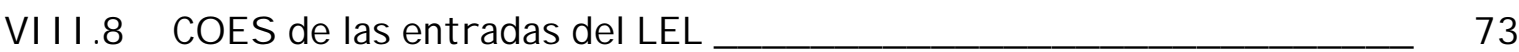

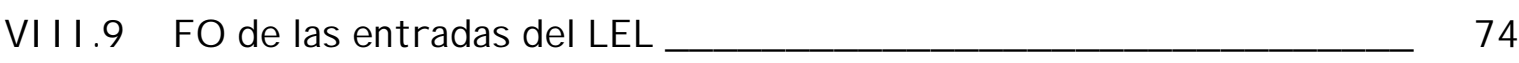

VII I.10 FO vs. FI de las entradas del LEL__-_-_-_-_-_-_-_-_-_-_-- 74

VIII.11 FOI de las entradas del $\mathcal{L E L} \mathcal{L}_{-}$-_-_-_-_-_-_-_-_-_-_-_-_-_-_- 76

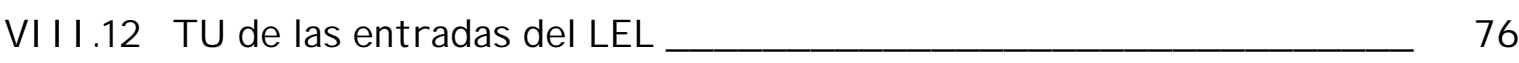

VIII.13 Contribución de cada tipo de palabra normalizada del LEL "Hotel"

VIII.14 Diagrama de dispersión y líne a de regresión para CPN vS. CP _-_-_-_ 86

VIII.15 Diagrama de dispersión y líne a de regresión para CPN vs. CVN $\mathcal{N}_{---} \quad 87$

VIII.16 Diagrama de dispersión y líne a de regresión para CPN vs. CS _-_-_ 87

VIII.17 Diagrama de dispersión y líne a de regresión para CPN VS. COPN__-- 88 
I I .1 Clasificación de las entradas

16

VIII.1 Mediciones extremas registradas para CPN

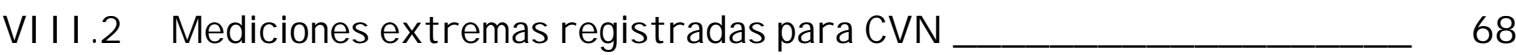

VIII.3 Mediciones extremas registradas para CS _-______-__-_-_-_-_ 69

VIII.4 Mediciones extremas registradas para COPN

VIII.5 Valores totales 77

VIII.6 Clasificación de las entradas del $\mathcal{L E L}$ "Hotel"

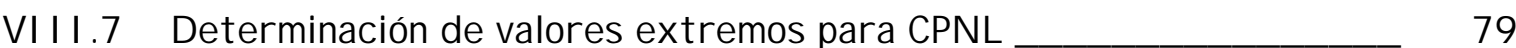

VIII.8 Determinación de valores extremos para las clases de palabras normalizadas

VIII.9 Comparación de mediciones de la tendencia central para el LEL "Hotel"

VIII.10 Mediciones de la tendencia central paralas categorias Verbo, objeto y $S$ uje to

VIII.11 Mediciones de la dispersión para las categorías y el LEL

VIII.12 Determinación del rango de valores del $75 \%$ de las mediciones según el Teorema de Che byshev.

VIII.13 Determinación del rango de valores del $68 \%$ de las me diciones según una distribución normal

VIII.14 Valores calculados para elcoeficiente de de terminación: $r^{2}$ 85

VII I.15 Mediciones efectuadas al $\mathcal{L E L}$ "Hotel"

IX.1 Valores obtenidos de las mediciones aplicadas al $L \mathcal{L} \mathcal{L}$ "Hotel"

IX.2 Valores obtenidos de las mediciones aplicadas al LEL "Pasaporte"

IX.3 Valores obtenidos de las mediciones aplicadas al $\mathcal{L E L}$ "LEL $\mathcal{E}$ Escenarios"

IX.4 Totales para la cantidad de palabras y cantidad de palabras normalizadas

IX .5 Distribución porcentual de las clases de palabras

IX.6 Valores de la dispersión para la cantidad de palabras normalizadas

IX.7 Valores paralos coeficientes de determinación para todas las clases de palabras con respecto a CPN

IX.8 Valores extremos, medios y encontrados para el $80 \%$ de las observaciones de la cantidad de palabras normalizadas

IX.9 Valores extremos, medios y encontrados para el $80 \%$ de las observaciones de los coeficientes Fan Out, FOI y Tasa de Ulso

IX.10 Valores extremos, medios y encontrados para el $80 \%$ de las observaciones de los coeficientes de redundancia y simplicidad 


\section{OBJETIVO}

Según [Fenton 96] hay dos razones fundamentales para realizar una investigación formal, ya sea un experimento, un caso de estudio o una recolección y posterior análisis de datos:

1. confirmar una teoría propuesta

2. determinar relaciones entre los datos

Esta tesis tratará de demostrar que un producto del proceso de elicitación de requerimientos como el Léxico Extendido del Lenguaje (LEL) es factible de ser medido. Para ello se proponen varias mediciones de tamaño que aplicadas al LEL, permitan mapear sus atributos y propiedades a un mundo de símbolos numéricos. Combinando estas mediciones se obtendrá un nuevo conjunto de valores que caractericen el artefacto en estudio.

La idea fundamental de la determinación del tamaño no se basa simplemente en la cantidad de palabras usadas que puede, ex profeso, ser muy pequeña en el afán de sintetizar el concepto al que se hace referencia o muy grande, para salvar ambigüedades, sino en la cantidad de palabras necesarias para explicar la idea subyacente. Es por esto que se busca establecer reglas de cuantificación que permitan abstraerse tanto de las singularidades del lenguaje como del estilo propio de cada analista y poder efectuar así mediciones objetivas.

\section{MOtivación}

Establecer algún tipo de medición que permita caracterizar un artefacto ayuda a controlar un proceso, si este control se realiza en etapas tempranas del desarrollo de un sistema se podrían hacer inferencias respecto a la factibilidad de continuar 0 no con el trabajo sobre bases más precisas.

Específicamente el LEL y los Escenarios que se pueden derivar en forma directa de él, son productos de una fase temprana de la Ingeniería de Requerimientos, la elicitación. Siendo esta etapa anterior a la especificación de los requerimientos si se lograra cuantificar este producto y si esa cuantificación permitiera inferir sobre la conveniencia de seguir con el proceso tal cual como está planteado o introducir cambios que mejoren la performance o directamente abandonarlo, el ahorro en tiempo y recursos, es evidente. 


\section{Metodología de trabajo}

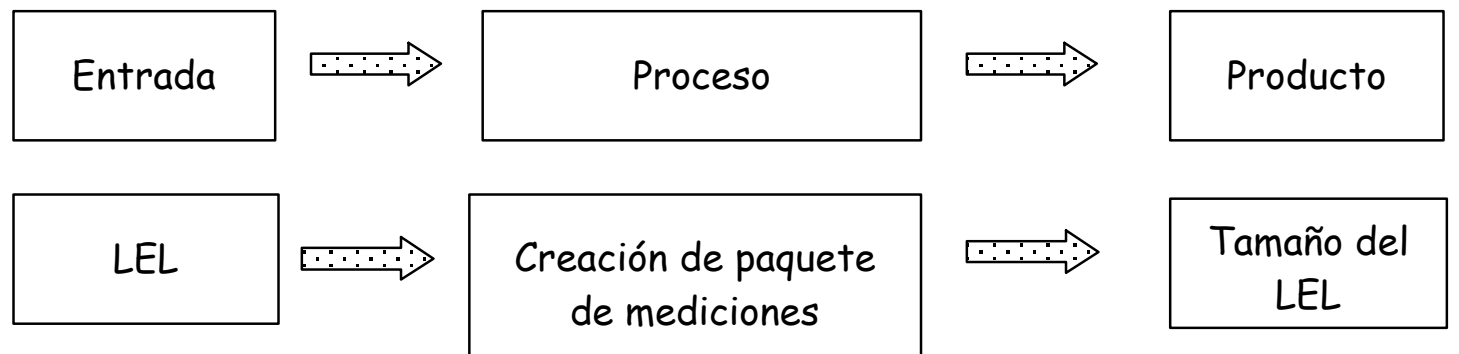

Figura I.1: Etapas de esta tesis

EI LEL es un conjunto de definiciones, al estilo de un diccionario de una actividad específica, está escrito en Lenguaje Natural y su estructura general está establecida en la bibliografía sobre el tema, por ejemplo [Hadad 97], [Leite 00].

El primer problema que se presenta radica en que las definiciones están expresadas en Lenguaje Natural y si bien su gramática y sintaxis dependen del idioma en el que se haya escrito, esta estructura no es lo suficientemente rígida como para establecer mediciones rigurosas ya que cada persona puede expresar la misma idea utilizando construcciones muy diferentes. Cuando el objetivo es medir tamaño de un texto escrito en Lenguaje Natural se deben establecer reglas para determinar parámetros de medición.

Por otro lado, se debe decidir qué elementos medir en un texto que resulten representativos de su tamaño. Las opciones son variadas: palabras o algún tipo de ellas, oraciones, signos de puntuación, espacios en blanco, líneas de texto, paréntesis - cualquier combinación de ellos. Esto lleva a la necesidad de jerarquizar los elementos medibles, que a su vez plantea la cuestión de establecer un criterio para realizar dicha jerarquización. Es fácil ver que muchos de estos elementos aportan poca información sobre el tema del texto, pero en cambio, otros parecen ser fundamentales.

Un estudio sobre la lingüística del idioma castellano permitió determinar las clases de palabras que pueden ser importantes en una definición. Con esta base se elaboran reglas de normalización que preservan sólo un conjunto de vocablos del texto que se pretende medir.

A partir de una investigación sobre la teoría de las mediciones tomando como base [Fenton 96], se construyó un paquete de definiciones de medidas internas y también algunas externas, específicamente diseñadas para el símbolo del LEL y para el LEL en su conjunto.

Se comenzó por las mediciones consideradas ingenuas como contar palabras y se llegó a otras más sofisticadas como la cantidad de veces que una entrada del LEL se usa para definir otras entradas.

Se proponen algunas soluciones, cuyo refinamiento se producirá en la medida que se avance en el desarrollo del trabajo.

El proceso seguido fue el siguiente: 
a) se decide medir tamaño contando la cantidad de palabras usadas en la construcción del LEL

b) luego de analizar las clases de palabras del lenguaje castellano, se estableció un concepto que permite estandarizar las mediciones: Palabra Normalizada. Una palabra se considera normalizada siempre que por su peso semántico sea imprescindible en la definición, es la que realmente transmite información.

Se formalizaron diez reglas que aplicadas a una palabra sucesivamente y sin seguir un orden fijo, permiten establecer su condición. Si fuera normalizada, se incluirá en la cantidad de palabras normalizadas, caso contrario, no se contará.

Esto surge del hecho de observar que aún tratándose de definiciones, en las que generalmente no se utiliza lenguaje coloquial, cada redactor tiene un estilo propio y por lo tanto utiliza una diferente cantidad y calidad de términos para expresar la misma idea. Entonces se tratará de reducir la cantidad de palabras a las que sean estrictamente necesarias para definir un concepto, prescindiendo así de palabras como preposiciones, conjunciones, artículos, pronombres, etc. que no aportan información importante.

c) se cuentan solo las palabras normalizadas de cada entrada obteniendo una medición llamada Cantidad de Palabras Normalizadas.

Para sistematizar esta actividad, se representan las definiciones utilizando un vector binario por cada oración de la entrada y que tendrá como dimensión la cantidad de palabras de la oración en donde cada posición contendrá un 1. Esta es una técnica similar a la utilizada en el área de la Recuperación de la Información [Faloutsos 95]. A medida que se aplican las reglas de normalización propuestas, algunas posiciones se cambiarán a 0 si la palabra que representa no está normalizada y por lo tanto se elimina. La suma de los elementos de todos los vectores dará el total de palabras normalizadas de la definición.

d) se establecen categorías de Palabras Normalizadas: verbos, símbolos del LEL y otras palabras normalizadas.

e) para cada símbolo se definen mediciones inmediatas o directas que incluyen la Cantidad de Palabras Normalizadas, la Cantidad de Oraciones, la Cantidad de Verbos Normalizados, la Cantidad de Otras Palabras Normalizadas, la Cantidad de Símbolos y cálculos utilizando esas mediciones, es decir, mediciones indirectas que incluyen Coeficientes de Redundancia y Simplicidad, el Fan Out, la relación entre Fan In y Fan Out y la Tasa de Uso.

f) para el LEL, se repite un proceso similar al del parágrafo anterior.

Se define la Cantidad de Símbolos que lo componen y la cantidad total de veces que se usaron verbos, símbolos del LEL y otras palabras normalizadas. Finalmente se realizan cálculos estadísticos que incluyen análisis de la tendencia central, de dispersión y de correlación. 
g) los procesos indicados en los apartados e) y f) se repiten para tres casos de estudio generándose así planillas de cálculo con los valores obtenidos y gráficos que muestran las tendencias observadas.

Estos casos de estudio fueron elaborados por grupos de personas diferentes, lo que asegura que no poseen modalidades propias de un individuo que pudiera imprimirles un sello personal y que además tienen una cantidad de entradas diferente. Se trata así de validar (o no) las mediciones propuestas y obtener conclusiones para todos los artefactos del mismo tipo.

h) se comparan los resultados obtenidos y se infieren características de tamaño y calidad para el LEL

Todo este conjunto de medidas expresadas en cantidades numéricas serán indicadores que permitirán caracterizar el LEL.

\section{PROpuestas RELACIONADAS}

Una investigación sobre el estado del arte de las mediciones aplicadas a productos del software permitió descubrir la falta de líneas de investigación en el campo de la aplicación de mediciones al LEL y Escenarios.

\section{ORGANIZACIÓN}

Esta tesis está dividida en diez capítulos, la bibliografía y una sección de apéndices. El primero es la introducción donde se explica someramente el objetivo.

El capítulo dos presenta conceptos sobre Elicitación de Requerimientos, Léxico Extendido del Lenguaje y Escenarios.

En el capítulo tres se explican algunos fundamentos de la Teoría de las Mediciones que son de gran importancia para la fundamentación de las técnicas utilizadas en esta tesis.

Para la realización del capítulo cuatro se contó con la colaboración de un lingüista que hizo valiosos aportes desde su especialidad para describir las características del Lenguaje Natural que permitirán justificar la reducción en la cantidad de palabras efectuadas a las definiciones del LEL.

En el capítulo cinco se explica el concepto de Palabra Normalizada en el contexto de esta tesis, la metodología utilizada para representar las definiciones del LEL y las diez reglas propuestas que se aplican a una palabra para determinar si es normalizada.

Los capítulos seis y siete definen las mediciones que se aplicarán a cada símbolo del LEL y al LEL en su conjunto.

En el capítulo ocho se presentan y analizan detalladamente las mediciones realizadas al LEL Hotel .

El capítulo nueve contiene el análisis de los resultados obtenidos para cada uno de los casos de estudio junto con un análisis comparativo de los tres 
En el capítulo diez se formulan las conclusiones finales y se establecen algunas pautas, derivadas de la tarea de haber medido estos LEL, para construir un LEL más fácilmente medible. Para terminar el capítulo se comentan futuros trabajos.

Por otro lado, la sección de apéndices incluye: en el apéndice $A$ las definiciones sobre las Clases de Palabras que se consideran al elaborar las reglas de normalización; en el apéndice $B$ se dan las Reglas de Normalización y en el apéndice $C$ las Definiciones de Mediciones Propuestas. El apéndice $D$ se dedica a explicar las fórmulas estadísticas elegidas para caracterizar el LEL. En el apéndice $E$ se incluye el LEL Hotel. Los apéndices $F, G$ y $H$ contienen las tablas donde se volcaron las mediciones inmediatas y los resultados de haber aplicado las mediciones calculadas utilizando la planilla de cálculo Excel 2000 de Microsoft a tres LEL que modelizan distintas actividades: la recepción de un hotel Hotel, el sistema nacional para la obtención de pasaportes Pasaporte [Leite 96] y la construcción del LEL y los escenarios LEL y Escenarios [García 00]. 


\section{CAPITULO $\|$}

\section{ELICITACIÓN DE REQUERIMIENTOS}

La Ingeniería del Software se puede definir según [Humphrey 90] como la aplicación disciplinada de principios, herramientas y métodos de ingenieria, ciencias y matemática para la producción económica de software de calidad. Para completar la definición anterior, el mismo autor llama proceso de la ingeniería de software al conjunto de todas las actividades de la ingeniería de software utilizadas para transformar los requerimientos del usuario en software.
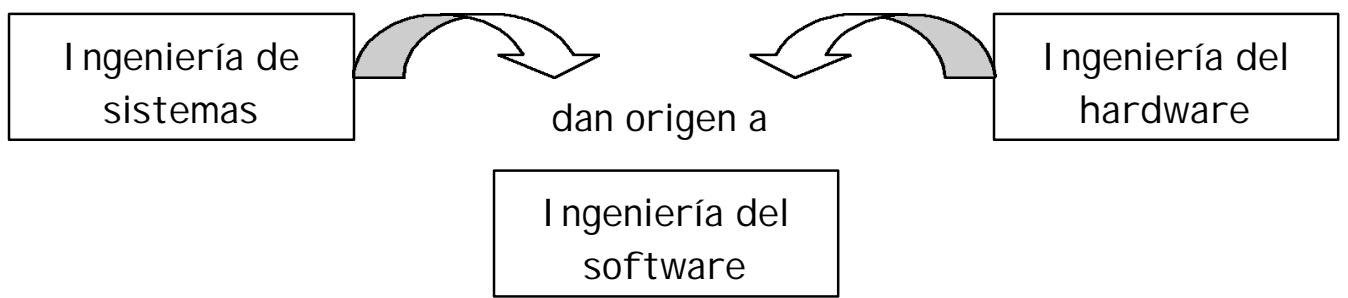

consta de tres fases

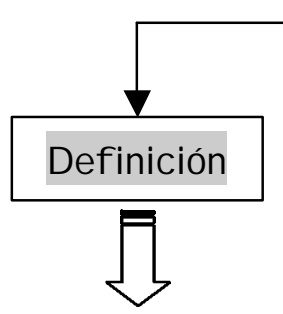

Anális is del siste ma

Planificación del proyecto

Anális is de reque rimientos

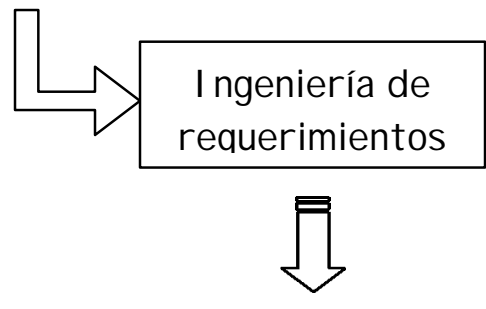
incluye los procesos

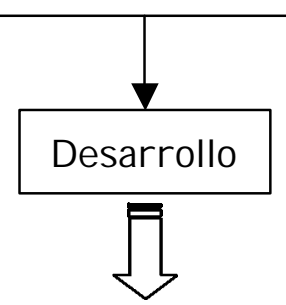

Diseño del software

Codificación

Prue 6 a del software

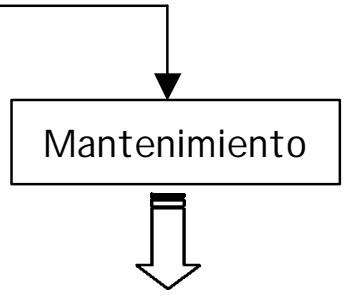

Corrección

Adaptación

Mejora
Elic itación Especificación Validación

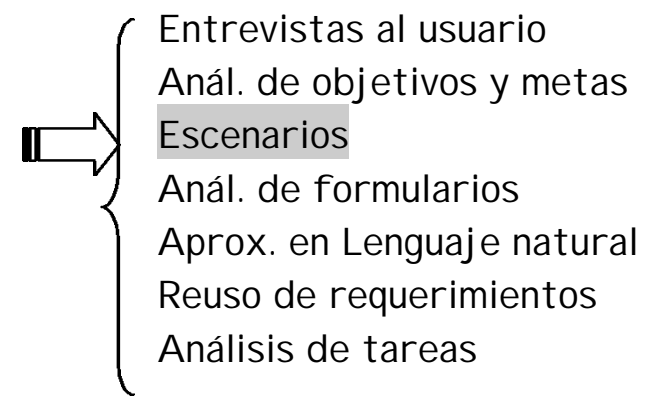

Figura II.1 Ubicación de los escenarios dentro de la IS (a partir de [Loucopoulos '95] y [Pressman'93])

Cada una de estas actividades será comprendida y controlada de forma tal de evitar cualquier imprevis to. 
Los métodos de la ingenie ría del software indican cómo construir técnicamente el software. Abarcan una amplia gama de tareas que incluyen análisis de requisitos, diseño, construcción de programas, prue bas y mantenimiento [Martinez'99].

Mientras que la ciencia de la computación provee los fundamentos teóricos para la construcción del software, la ingeniería del software se centra en su implementación en forma controlada y cientifica [Fenton'96].

\section{INGEDI ERI A DE REQ UIERIMI ENTOS}

Es bien sabido que una evaluación deficiente, errónea o incompleta en las etapas tempranas del desarrollo de un sistema produce inconvenientes que se encuentran entre los más costosos de reparar cuando el sistema ha sido implementado y en el peor de los casos lleva a desecharlo después de que ha sido comple tamente implementado [Doorn' 98 ].

Es por lo antedicho que una actividad central en el desarrollo de los sistemas de software es la correcta interpretación de las necesidades de los clientes-usuarios. Las expectativas que ellos tienen sobre el sistema de software se formalizan al establecer la especificación de los requerimientos. La rama de la Ingeniería del Software que se ocupa de esta actividad, se conoce como Ingeniería de Requerimientos [Loucopoulos 95 ].

Es común que se produzcan fallas al especificar los requerimientos del sistema en análisis. Estas fallas según [Christel'93] se deben a varios factores, entre ellos:

- resulta dificil crear modelos estructurados a partir de las descripciones en lenguaje natural de los clientes-usuarios

- en la transición entre los requerimientos en bruto a las especificaciones formales, se pierde información potencialmente importante y el traceability ${ }^{1}$ se torna dificil de mantener

- La información sobre políticas, procesos y metodologías debería estar disponible, pero muchas veces el acceso a ella es dificil

Es probable que los requerimientos se modifiquen muchas veces durante el desarrollo del sistema, antes de llegar al diseño definitivo, esto involucra una sucesión de proposiciones que se van acercando a las percepciones de los usuarios finales acerca delobjetivo del sistema [Loucopoulos '95].

Todo sistema típico contiene cientos de requerimientos que derivan de diferentes fuentes y en formatos diversos, lo que produce una gran cantidad de información compleja y sin procesar a partir de la cual se deben especificar los requerimientos [Christe['93].

La Ingeniería de Requerimientos es una disciplina relativamente nueva y por lo tanto existen distintas corrientes, cada una de ellas adopta sus propias normativas en las tres actividades fundamentales que involucra, según [Loucopoulos 95]:

1. elicitación: obtener todo el conocimiento posible acerca del problema y documentar los hechos observados

2. especificación: formalizar el conocimiento adquirido del Udd (Universo de discurso) en forma de características que el sistema debe tener o cumplir

\footnotetext{
${ }^{1}$ Rastreabilidad: habilidad de rastrear. En general se prefiere usar el vocablo en inglés
} 
3. validación: verificar la precisión de la comprensión obtenida

\section{ELICITACIÓN DE REQ UERI MIENTOS}

En este mundo complejo y competitivo, donde la información se considera poder, es una ardua tarea llegar a conocer hasta en sus más minimos detalles la forma de trabajar de una organización o empresa. En el marco de la Ingeniería de Requerimientos, se denomina elicitación a: "El proceso de adquisición (elicitación) de todo el conocimiento relevante necesario para producir un modelo de requerimientos del dominio de un problema" [Loucopoulos 95 ].

$\mathcal{E l}$ ingeniero de requerimientos debe ser capaz de obtener los requerimientos del sistema a partir de una amplia variedad de clientes-usuarios. Muchas veces esos requerimientos son confusos, vagos y están expresados como soluciones [Noseibeh 97 ].

La elicitación de requerimientos es una actividad netamente social, donde la interacción entre los usuarios, el medio y los analistas de be ser fluida y cordial para llegar a la transmisión de conocimientos que permitan la especificación de los requerimientos que solo tienen sentido dentro del contexto (tiempo, lugar y situaciones) en elque fueron establecidos [Loucopoulos '95].

Más aún, si el trabajo lo realizan varias personas y alguna de ellas no acue rda o teme a posibles cambios, la tarea se torna muy dificil o casi imposible. Siempre que exista interacción entre diferentes grupos de interés, se pueden generar situaciones conflictivas que derivan en falta de entendimiento, falta de colaboración, malas interpretaciones o simplemente información erróne a o poco exacta.

"La calidad de la especificación de requerimientos y en última instancia del sistema de información de pende mucho de la habilidad del desarrollador para extraer y comprender el conocimiento acerca del dominio modelado, el universo del discurso" [Loucopoulos 95 ].

$\mathcal{E l}$ universo del discurso, ZldD, ambiente de trabajo, ambiente a modelizar, dominio de análisis o macrosiste ma se usarán como sinónimos para referirse al espacio físico, temporal y condiciones en general en las que se desarrollará el proceso de elicitación y por ende se implantará el sistema de software terminado.

Es importante que los propios desarrolladores analicen el problema con los usuarios finales y no que reciban las interpretaciones de un grupo especializado que, muchas veces, los clientes designan para interpretar los requerimientos de la empresa y brindar la información de forma de no alterar la productividad y el normal desarrollo del trabajo. Esto puede ser fuente de grandes confusiones y malas interpretaciones.

Es por esto que la etapa de elicitación de requerimientos es muy sensible a las personas que entran en contacto. Por un lado los desarrolladores del sistema que pueden tener preconceptos o quizás un total desconocimiento sobre el ambiente que tienen que investigar y por otro lado los clientes o potenciales usuarios que, como se mencionó antes, pueden estar ansiosos por introducir innovaciones en su ambiente de trabajo o bien temerosos de perder privilegios y por lo tanto te men a los cambios. En el medio de estas posiciones extremas hay un sinnumero de posibilidades que pueden conducir a una especificación de requerimientos poco precisa. 
"...la elicitación de requerimientos es una actividad crucial en el desarrollo de sistemas que está comúnmente plagada de problemas de traceability, inadecuada detección y resolución de conflictos, pobre comunicación entre los desarrolladores y los clientes-usuarios y la pérdida de información importante sobre el proceso y el sistema" [Christel'93].

Un buen trabajo en la e tapa de elicitación de requerimientos es determinante para el éxito del proyecto, pues un sistema que se desarrolla en base a requerimientos inexactos o erróneos, producirá software poco útil.

Dentro de la literatura sobre elicitación se proponen diversas técnicas para extraer conocimiento del ambiente a modelizar o Universo del Discurso (UdD). Según [Loucopoulos 95], se pueden considerar las siguientes:

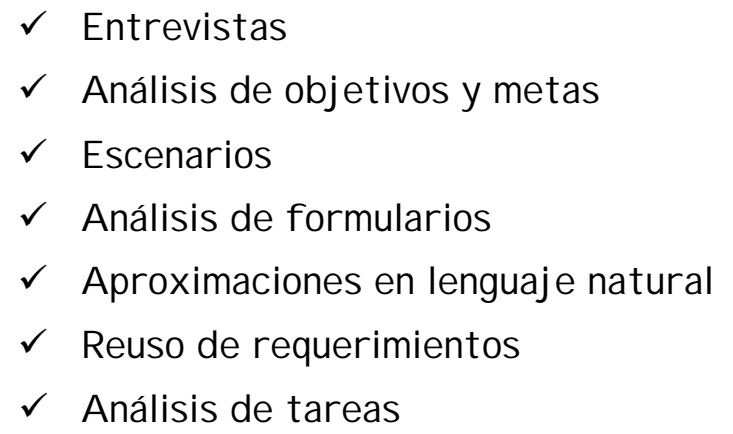

En esta tesis se ha usado la técnica de Escenarios generados a partir del Léxico Extendido del Lenguaje (LEL), tal como se propone en [Hadad 97]. En la práctica esta elección conlleva al uso de otras técnicas que ayudan a la elicitación, a saber: Entrevistas, Aproximaciones en lenguaje natural y Análisis de tareas, pero todas van en auxilio de la creación de escenarios.

"Las técnicas de escenarios para la elicitación de requerimientos se basan en el hecho que los usuarios encuentran más fácil transferir sus pericias al analista a través de una sesión activa contando una historia, que a través de cuestionarios y entrevistas... las técnicas de escenarios presentan una solución promisoria para el difícil problema de comunicación y transferencia de las pericias entre el analista y el usuario" [Loucopoulos '95].

\section{MEDICION DE REQ UERI MI ENTOS}

En cualquier actividad que se esté desarrollando es importante plantear la necesidad de medir el proceso para intentar mejorarlo y el producto para intentar mejorar su calidad pues si no se mejora significa que las estrategias de trabajo usadas no son convenientes.

Las mediciones aplicadas a los requerimientos son importantes por los beneficios asociados a la detección y corrección temprana de problemas [Hammer $97]$.

Para que los requerimientos sean útiles deben ser medibles en alguna forma [Robertson 97]. Un prerrequisito obvio para aceptar y usar una medición es que realmente mida la característica del software que se supone que mide [Briand 96] 
Los pasos que se sugieren en [Pressman 93] para implementar un programa de mediciones son:

1. Recolección de datos

2. Cálculo de métricas

3. Evaluación de los resultados

Las mediciones del software se utilizan según [Zuse '95] y [Grady '94] para medir atributos especificos del producto o del proceso de desarrollo y permiten:

1. Establecer bases para una estimación

2. Monitorear el progreso de un proyecto

3. Determinar la complejidad

4. Saber cuándo se ha llegado al grado de calidad dese ado

5. Evaluar los productos

6. Analizar defectos para mejorar el proceso

7. Validar experimentalmente las mejores prácticas

En pocas palabras, ayudan a tomar mejores decisiones.

$\mathcal{E}$ léxito de un programa de mediciones está fuertemente ligado a la elicitación, definición y manipulación tanto de las entidades y actividades del modelo empirico del mundo real que se quiere medir como de su modelo numérico formal [Offen '97].

Si es posible cuantificar los requerimientos y auditar los procesos de medición usados junto con los resultados obtenidos, entonces se puede asegurar la calidad de los requerimientos producidos. En base a estas mediciones se puede entonces evaluar el software, negociar contratos y especialmente controlar las fallas [Rule '01].

Entender el tamaño del producto es crucial para entender el proceso de software y para controlar restricciones del proceso tales como duración y productividad, junto con otros factores que afectan la satisfacción delcliente [Rule '0 1].

\section{4. $\mathcal{E S C E N A R I O S ~}$}

"Los escenarios son descripciones breves de situaciones típicas en el uso del sis te ma" [B̈riand'98].

Según la definición dada en el diccionario [Marín '82] se denomina escenario al "Conjunto de circunstancias que se consideran en torno de una persona o suceso"

Un escenario describirá detalladamente situaciones relevantes que se dan en el ambiente para el cual se debe elaborar la especificación de requerimientos. "Los escenarios son descripciones parciales del funcionamiento del sistema, focalizan un momento específico de la aplicación ... presentan versiones acotadas del comportamiento delsoftware "[Leite 96].

"Lo que se representa en un escenario varia desde descripciones concretas de la realidad hasta sistemas diseñados que se ejecutan como simulaciones para presentar una visión de cómo se va a comportar el sistema en el futuro "ISutcliffe 97 ]. 
"La creación de escenarios hace, generalmente, necesaria la adquisición de conocimiento del dominio" [S utcliffe '97]. Los escenarios proveen una forma natural de obtener y presentar el conocimiento que tienen del macrosistema cada uno de los participantes del proyecto, una vez que este conocimiento queda modelizado, la especificación de los requerimientos es un paso posterior de fácil implementación. "los escenarios son una herramienta importante para el ingeniero de requerimientos, no solo por la forma natural en la que describe el comportamiento, sino también porque obliga el ingeniero de sistemas a mapear el macrosistema en el que trabajará elfuturo software" [Leite '97].

"existe una gran variedad de medios para presentar los escenarios: textos en lenguaje natural e imágenes o formas simbólicas como diagramas, notaciones formales, etc. Aún más, el contenido que se representa pueden ser hechos registrados en algún medio o un artefacto diseñado que entrega su propia presentación como una simulación" [S utcliffe 97]. En este trabajo se elige una representación en texto en lenguaje natural de las situaciones que el desarrollador describe a partir del conocimiento que adquirió del UddD y que validó con los clientes. usuarios del sistema.

Los escenarios tienen las siguientes características generales, según [Leite 97]:

- describen situaciones en el macrosistema

- evolucionan junto con el proceso de construcción del software

- están naturalmente enlazados al LEL

- describen situaciones haciendo énfasis en el comportamiento usando lenguaje natural

En [Wilson 99] se recomienda a los analistas, entre otras cosas, que al usar lenguaje natural para especificar los requerimientos del sistema utilicen oraciones simples y directas cuya estructura sea correcta y seleccionen palabras y frases basadas en una definición formal y no en lo que el uso común supone que significan.

La siguiente etapa se ocupará de describir el conjunto de palabras de uso corriente entre los clientes-usuarios del sistema que se denomina Léxico Extendido del Lenguaje (LEL) y posteriormente se obtendránlos escenarios a partir de él como se sugie re en [Doorn'98], [Hadad'97].

Una ve z consensuada con las partes intervinientes (desarrolladores y clientes. usuarios), la lista de simbolos del LEL sirve de base para la generación de los escenarios derivados de ellos.

\section{LÉXICO EXTENDIDO DEL LENGUAgE}

El Léxico Extendido del Lenguaje (LEL) permite analizar el vocabulario propio del dominio en análisis y asi lograr unificar los términos para el intercambio de información ("hablar el mismo idioma") entre los clientes-usuarios y los desarrolladores, ".. permite conocer elvocabulario de la aplicación, tal como lo utiliza el usuario. "[Leite 96], restringiendo la cantidad de vocablos posibles para evitar ambigüedades. 
Resulta útil conocer la definición de la palabra léxico para los lingüistas: "especie de diccionario o glosario en cuyas entradas se especifican las propiedades idiosincrásicas ${ }^{2}$ de las pie zas o elementos léxicos de una lengua" [Lagunilla 95]. Ve mos que concuerda exactamente con las características que se espera que posean las entradas del LEL generado durante el proceso de elicitación.

En esta e tapa se utilizará el lenguaje natural, aquélque es de uso cotidiano en elambiente de trabajo en el que los desarrolladores están adquiriendo conocimiento. Ellos están aprendiendo sobre un macrosistema que no conocen profundamente y esto puede crear "gaps" semánticos entre las partes involucradas (desarrolladores y clientes-usuarios) que irán desapareciendo en sucesivas entrevistas. "Una forma de facilitar la comprensión es a través del uso del lenguaje natural, evitando la incorporación de un lenguaje artificial ya desde el comienzo del proceso" [Leite 96]. Es decir, las entrevistas no se realizan utilizando términos técnicos propios del análisis de sistemas, sino solamente los utilizados en el ambiente que se está estudiando.

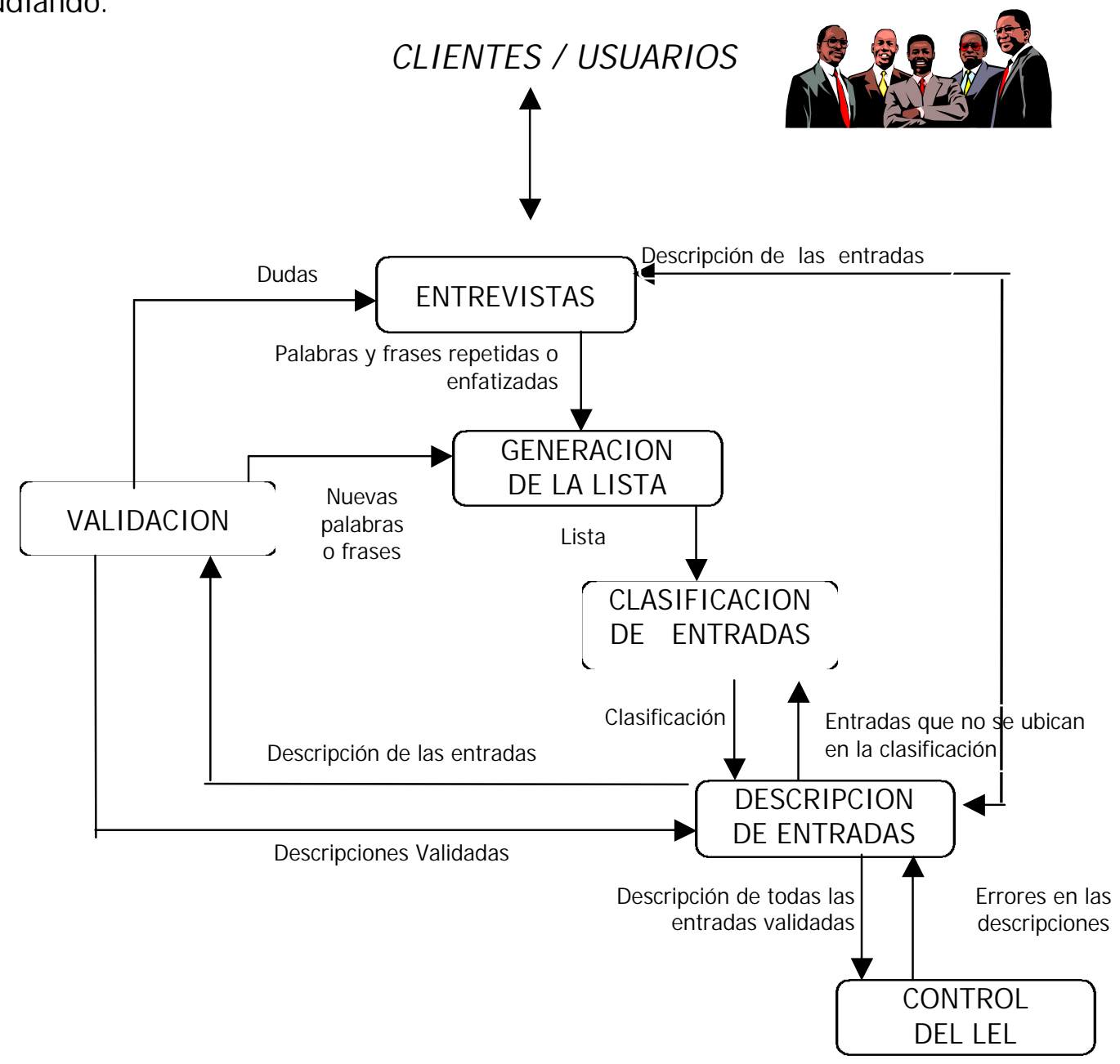

Figura I I.2: Pasos para la construcción del LEL [Hadad 97]

\footnotetext{
2 Idiosincrásico: perteneciente o relativo a la idiosincrasia.

Idiosincrasia: indole del temperamento y carácter de cada individuo por lo cual se distingue de los de más.
} 
$\mathcal{E l}$ desarrollador, en una primera etapa, mantiene charlas con los clientes. usuarios del sistema para obtener conocimiento acerca del UdD. Estas entrevistas informales permiten identificar las palabras más usadas y suexacto significado en el entorno en estudio, "También se incluyen aquéllas palabras o frases que son relevantes para el dominio del problema más allá de su frecuencia de repetición" [Leite 96]. Estas palabras conformarán el LEL, se consigue asíque desarrolladores y clientes-usuarios utilicen las mismas palabras con idéntico significado. Los desarrolladores no sólo obtendrán conocimiento a través de los clientes-usuarios, sino también observando el ambiente de trabajo, consultando bibliografia sobre el tema, leyendo informes generados por los clientes-usuarios y de toda aquélla fuente que pueda transmitirles conocimiento. Cada vocablo o palabra más usada en el macrosistema se incluye en la lista y se denominará entrada o simbolo del $\mathrm{LEL}^{3}$. Tendrá un nombre único que lo identifica, pero puede tener sinónimos que irán separados por barras.

Se pretende que esta lista incluya la menor cantidad posible de palabras externas al dominio, es lo que se denomina "principio de vocabulario minimo" [Leite $96]$.

Es común encontrarse en la situación de tratar de establecer la noción y/o impacto de un simbolo, utilizando otros simbolos pertenecientes al sistema en estudio, esto se denomina "principio de circularidad", se genera asi una red de interacciones y dependencias entre los simbolos que es altamente beneficiosa [Leite 96]

Los simbolos se clasifican en $S$ ujeto, Verbo, Objeto y Estado [Hadad 97], pero esta clasificación general se adaptará a cada Udd particular. Pue de ocurrir que se generen nuevas categorias que sean especializaciones de las anteriores o bien una clasificación que ejemplifique mejor las características de los simbolos que engloba.

\section{APLICACIÓN AL CASO DE ESTUDIO}

El trabajo de campo consistió en el anális is delfuncionamiento de la Recepción del "Hotel Lucania" ubicado en la ciudad de Comodoro Rivadavia. Este hotel de cuatro estrellas, el de mayor categoría de la ciudad, resultó un desafío de gran interés para los desarrolladores.

En principio, un frotel se presentab a como una tare a faraónica, casi imposible de analizar, compuesta por varios departamentos, cada uno con su actividad especifica y diferente. Sin embargo, después de las primeras entrevistas con un recepcionista del hotel, se logró comprender que el control de casi todas las actividades se centraba en la Recepción, lo cual ayudó a circunscribir el proble ma sin perder por ello la visión de conjunto.

Se produjeron cuatro versiones de LEL que fueron evolucionando junto con el conocimiento de los desarrolladores sobre el UddD.

Una condición para poder trabajar en el Hotel fue entrevistar al recepcionis ta del turno noche (de afora en adelante usuario), pues es en ese momento cuando se registra menos movimiento en la Recepción.

\footnotetext{
${ }^{3}$ En este trabajo se utilizará indistintamente las palabras simbolo del LEL y entrada del LEL

"El Apéndice E contie ne una descripción comple ta del LEL "Hotel"
} 
Para la primera reunión se prepararon algunas preguntas elaboradas después de leer la bibliografía sobre LEL y Escenarios, pero sin pretender establecer una entrevista estructurada. Se generó así una lista de palabras propias del UddD o, lo que llamaremos, simbolos candidatos del LEL.

Estos simbolos se agruparon según una clasificación adoptada que difiere ligeramente de la clasificación general propuesta en [Hadad'97].

La lista de simbolos candidatos, que en un principio resultó muy extensa, fue sometida a validación con el usuario en sucesivas entrevistas. Como resultado del proceso de validación, muchos simbolos candidatos se rechazaron definitivamente, otros cambiaron su contenido y algunos se agregaron. En resumen, los simbolos evolucionaron junto con el proceso de crecimiento delconocimiento del macrosistema hasta llegar a la lista definitiva.

Para cumplir con el "principio de vocabulario minimo", se tuvo especial cuidado de no caer en la tentación de ampliar los simbolos haciendo aportes personales de los desarrolladores y ajustándose estrictamente a las palabras con las que describia las ac tividades el usuario.

El identificador de cada una de las entradas del LEL surgió naturalmente a instancias del usuario, sólo hubo que prestar atención a los posibles sinónimos para asegurarse que no fueran un simbolo diferente. El orden en el que se establecieron los sinónimos no refleja necesariamente la frecuencia de uso.

Para describir cada simbolo se utilizan la Nocióx donde se explica brevemente qué significa esa palabra en el contexto en estudio y el IMPACTO, que puede faltar, define las acciones que ese simbolo ejecuta y que incidirán en los otros componentes del sistema pudiendo alterarlos en función de su comportamiento.

$\mathcal{E} j$.

Pasajero / huésped / pax

- Notion

- Es una persona que tiene una solicitud de reserva o hace un pedido de alojamiento

- Behavioral responses

- Provee los datos requeridos por el recepcionista.

- Puede presentar un voucher.

- Completa la planilla del pasajero la primera vez que se presenta en el Hotel.

- Recibe la tarjeta codificada y el control remoto TV.

- Puede solicitar la llave de la caja de seguridad y/o minibar.

- Puede hacer consumos en Cafetería y/o Restaurant, consumos del minibar, llamados telefónicos y gastos de lavandería.

- Puede hacer un pedido del servicio de despertador.

- Puede hacer un pedido de limpieza extra y/o un pedido de mantenimiento extra.

- Puede recibir un aviso de mensaje.

- Recibe la factura y los comprobantes de gastos.

- Hace el pago.

- Devuelve la tarjeta codificada, el control remoto TV y la llave del minibar y/o de la caja de seguridad de la habitación si las solicitó.

- Puede hacer una cancelación de reserva. 
Todas las palabras que aparecen subrayadas en el ejemplo son simbolos del LEL usados para la definir el simbolos del LEL pasajero/huésped/pax, se cumple así el "principio de circularidad". Esta red de interacciones y dependencias entre los simbolos obliga a prestar mucha atención cuando se altera alguno de ellos porque influirá en otros.

Para paliar esta dificultad y no perder el control de los cambios, es que se recomienda el uso de alguna herramienta que ayude en la actualización de los simbolos del $L \mathcal{E L}$.

En este trabajo, en un primer momento, se usó el procesador de texto Word del paquete Office 2000 de Microsoft que permite establecer hipervinculos entre las palabras. Si bien resultó de gran ayuda para navegar entre simbolos, los cambios resultaban difíciles de implementar, pues se deben rescribir todas las apariciones del simbolo.

Finalmente se utilizó la herramienta Baseline Mentor Workbench (BMW) "Esta es una aplicación construida para asistir al experto del dominio durante la fase de ingeniería de requerimientos utilizando el esquema del "client-oriented baseline" [Antone lli 01$]$.

Los simbolos se clasificaron según el siguiente detalle tomando como base [Kaplan 96] y atendiendo a las particularidades del macrosistema en estudio:

Actividad (A): en la noción se indica en qué consisten, quién las ejecuta y el lugar donde se realizan y en el impacto se describe el efecto que producen sobre Datos, Formularios, Personas, Objetos, Ficheros.

Dato (D): en la $\mathcal{N}$ (oción se indica su contenido, qué actividad o función los provee y dónde se almacenan y en el Impacto se describen las actividades en las que se usan.

De partamento $(\mathcal{T})$ : en la $\mathcal{N}$ (oción se indican las actividades que realiza y en el Impacto se describen los efectos sobre Personas, Formularios, Datos, Ficheros, Objetos.

Fichero (F): en la Noción se indica su contenido y ubicación y en el Impacto se establece en que actividades se utilizan.

Formulario (M): en la Noción se indic a su contenido, qué actividad o función los provee y dónde se almacenan y en el Impacto se describen las actividades que se realizan sobre ellos.

Objeto $(O)$ : en la Noción se define el concepto, sus características, su utilidad y su ubicación y en el Impacto se describen las actividades que se realizan sobre ellos.

Persona (P): en la Noción se definen sus características y en el Impacto se explica cuál es su acción sobre Datos, Actividades, Formularios, Ficheros, Objetos.

En la Tabla I I.1 figura la cantidad de entradas que corresponden a cada grupo según ambas clasificaciones para mostrar qué categorias de la clasificación particular se incluyeron en la clasificación ge neral y cómo se distribuyó la cantidad de entradas en cada categoría. 


\begin{tabular}{|c|c|c|c|}
\hline Clasificacion particular & Cantidad & Clasificacion general & Cantidad \\
\hline$\checkmark$ Actividad & 12 & $\checkmark$ Verbo & 12 \\
\hline$\checkmark$ Dato & 4 & \multirow{5}{*}{$\checkmark$ objeto } & \multirow{5}{*}{25} \\
\hline$\checkmark$ Departamento & 7 & & \\
\hline$\checkmark$ Fichero & 4 & & \\
\hline$\checkmark$ Formulario & 7 & & \\
\hline$\checkmark$ objeto & 5 & & \\
\hline$\checkmark$ Persona & 2 & $\checkmark$ Sujeto & 2 \\
\hline \multicolumn{3}{|l|}{$\mathcal{T} O \mathcal{T A} \mathcal{L}$} & 39 \\
\hline
\end{tabular}

Tabla I I.1: Clasificación de las entradas

Como las categorías \{Persona-S uje to\} y \{Actividad -Verbo\} son equivalentes en cuanto a su definición, poseen la misma cantidad de ele mentos.

En la Figura II.3 se puede apreciar la distribución porcentual de las entradas del LEL en función de la clasificación adoptada que se muestra en la Tabla II.1. Es interesante observar que los resultados se corresponden a los esperados según el ambiente que se está modelizando.

El mayor peso está en el rubro Actividad y es justamente las acciones que se desarrollan en la Recepción del Hotel lo que se quiere describir. Le sigue Departamento que en un primer momento no parecería tan relevante, pero haciendo un mejor análisis vemos que son dependencias controladas por la RECEPCIÓN y finalmente el otro item de peso es Formulario, nuevamente si se piensa que la información se manipula con material escrito, resulta muy obvia su importancia. Algo para destacar es que Persona con un porcentaje 6ajo, es muy importante, las actividades se resumen en el intercambio entre el pASAgERO, que es la razón de la existencia del Hotel y el RECEPCIONISTA que es la única persona con la que interactúa. El resto de los empleados resultan seres anónimos para esta aplicación.

\section{PERSONA}

$3 \%$

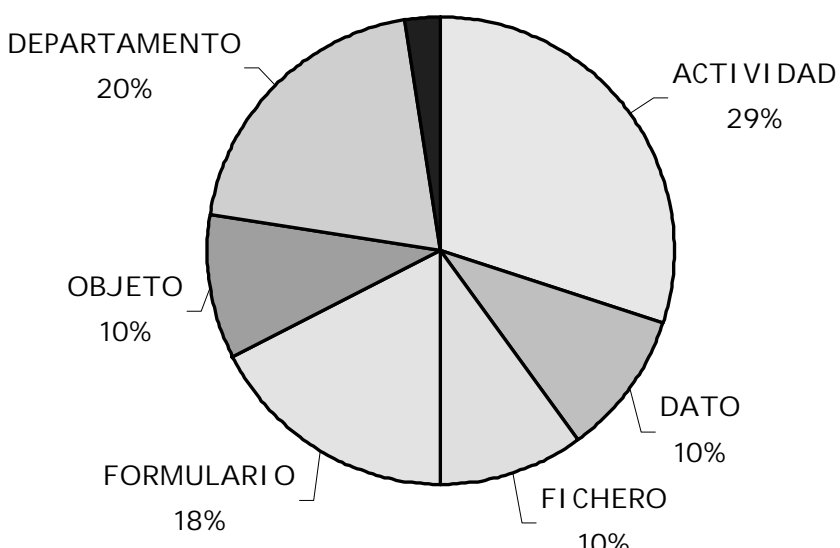

$10 \%$

Figura I I.3: Distribución según la clasific ación particular 
Con la clasific ación general, como puede apreciarse en la Figura II.4, el mayor peso recae en la categoría Objeto, lo cuales previsible, sobre todo tratándose de un siste ma administrativo. La categoría Estado está ausente de este análisis pues no se trata de un sistema en tiempo real.

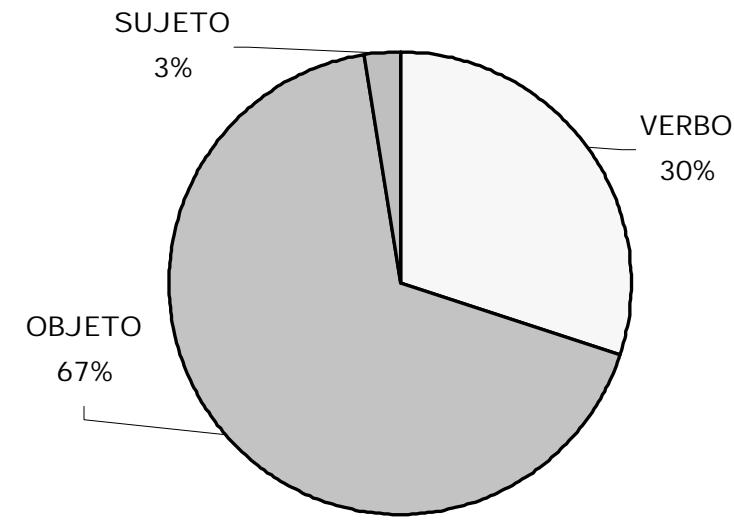

Figura I I.4: Distribución según la clasific ación ge ne ral

En cuanto a esta clasificación, se puede decir que se adapta a la clásica de objeto, Sujeto, Verbo y Estado, pero se especializa dentro de la categoría Objeto ampliándola a Fichero, Formulario, Dato y Objeto, pues en la actividad hotelera se encuentra una profunda diferencia entre ellos.

Ej. Obje to

minibar

- Notion

- Es una pequeña heladera que tiene una llave.

- Se utiliza para conservar bebidas y alimentos refrigerados.

- Está en cada habitación.

- Behavioral responses

- El pasajero / huésped / pax puede consumir los elementos que contiene.

- Diariamente el Departamento mucamas controla su contenido y repone los elementos faltantes.

Ej. Fichero

planilla de ocupación de habitaciones

- Notion

- Contiene la solicitud de reserva y ocupación según el tipo de habitación.

- Se guarda en la recepción.

- Behavioral responses

- Se actualiza cuando se hace una solicitud de reserva, una cancelación de reserva, un no show, un check in o un check out.

Ej. Formulario

comprobante de llamados telefónicos

- Notion

- Contiene el importe de la llamada telefónica y el número de habitación del pasajero / huésped / pax. 
- Lo provee el sistema telefónico.

- Se almacena en la carpeta de la habitación.

- Behavioral responses

- El sistema telefónico lo hace en forma automática.

Ej. Dato

tarifa

- Notion

- Es el costo de la habitación según sus características.

- La provee la lista de precios.

- Se almacena en la lista de precios.

- Behavioral responses

- La utiliza la administración para hacer la facturación.

Persona equivale a Sujeto (ver ejemplo de Pasajero / huésped / pax) y Actividad a Verbo.

Ej. Actividad

pago

- Notion

- Es el proceso para cancelar el importe de la facturación al pasajero / huésped / pax.

- Lo hace el pasajero / huésped / pax.

- Se hace en la recepción.

- Behavioral responses

- Si es en efectivo o cheque se guarda en la caja de la recepción.

- Si es con tarjeta de crédito se solicita la autorización, se hace el cupón, el pasajero / huésped / pax lo firma y se guarda en la recepción.

- Si se debita de una cuenta corporativa el pasajero / huésped / pax firma la factura y se envía a la administración.

Por otro lado se agrega la clasificación Departamento y no se utiliza Estado porque no se trata de un sistema en tiempo real.

Ej. De partamento

Recepción

- Notion

- Es un departamento interrelacionado con todos los departamentos del Hotel.

- Atiende todas las actividades relativas al ingreso, permanencia y egreso del pasajero/huésped/pax.

- Behavioral responses

- Es el lugar de trabajo del recepcionista.

- Almacena toda la información relativa al movimiento de pasajero / huésped / pax.

Los simbolos definitivos que describen las actividades propias de la Recepción y por ende, según la visión del conjunto, de casi todo el Hotel son los siguientes: 


\section{Actividades}

CANCELACION DE RESERVA

CHECK IN

CHECK OUT

FACTURACIÓN

NO SHOW

PAGO

PEDIDO DE ALOJAMIENTO

PEDIDO DE LIMPIEZA EXTRA

PEDIDO DE MANTENIMIENTO EXTRA

PEDIDO DEL SERVICIO DE DESPERTADOR

REPOSICION DE INSUMOS

SOLICITUD DE RESERVA

Datos

DIA DE EGRESO

DIA DE INGRESO

DISPONIBILIDAD DE HABITACIONES

TARIFA

Departamentos

ADMINISTRACIÓN

AGENCIA DE VIAJES / AGENCIA

GASTRONOMIA

MANTENIMIENTO

MUCAMAS

RECEPCIÓN

Ficheros

CARPETA DE LA HABITACIÓN

FICHERO DE PASAJEROS

PLANILLA DE OCUPACION DE HABITACIONES

PLANILLA DE RESERVAS

Formularios

AVISO DE MENSAJE

COMPROBANTE DE CONSUMOS DEL MINIBAR

COMPROBANTE DE CONSUMOS DE CAFETERIA Y/O RESTAURANT

COMPROBANTE DE LAVANDERÍA

COMPROBANTE DE LLAMADOS TELEFONICOS

PLANILLA DEL PASAJERO

VOUCHER

Objetos

HABITACION

LISTA DE PRECIOS

MINIBAR / FRIGOBAR

TARJETA CODIFICADA

Personas

PASAJERO / HUÉSPED / PAX

RECEPCIONISTA 
Se siguió el esquema sugerido en [Hadad 97] que propone heurísticas para la construcción de escenarios a partir del $L \mathcal{L L}$.

Como primer paso, se identificaron los actores principales y secundarios de la aplicación, "Actores: personas o estructuras organizacionales con un papel en el escenario" [Hadad'97].

En este ejemplo son:

Principales:

Secundarios:

$\underline{\text { Recepcionista }}$

pasajero / huésped / pax

Departamento administración

Departamento gastronomía

Departamento mantenimiento

Departamento mucamas

A continuación se hace una lista con algunos de los impactos de los simbolos correspondientes a los actores principales identificados que se amplía con los impactos de los actores secundarios y se eliminan aquellos que estén repetidos:

Atiende una solicitud de reserva o un pedido de alojamiento.

Hace el checkin.

Atiende el pedido del servicio de despertador.

Recibe el pedido de limpieza extra y elpedido de mantenimiento extra delpasajero / huésped / pax.

Hace el pedido de reposición de insumos.

Hace el check out.

Ante la cancelación de reservas o el no showactualiza la planilla de reservas.

Hace el pago.

Estos impactos darán origen a la lista de escenarios candidatos. Cada uno de ellos se describe utilizando el siguiente esquema [Leite 97]:

Nombre: titulo delescenario, puede ser el mismo que se usó para una entrada del LEL Objetivo: finalidad a ser alcanzada en el contexto del problema.

Contexto: ubicación geográfica/temporal y/o estado inicial delescenario.

Recursos: medios de soporte, dispositivos u otros elementos pasivos que deben estar disponibles en el escenario.

Actores: personas o estructuras organizacionales con un papelen el escenario.

Episodios: acciones que de tallan al escenario y proveen su comportamiento.

Se vio la necesidad, en algunos casos, de agregar dos elementos descriptivos más [Leite 96]:

Restricciones: situaciones que impiden la concreción delescenario.

Casos alternativos: hace mención a otras situaciones que impiden que se ejecute el escenario.

En las restricciones se expresan los requerimientos no funcionales y en los episodios y los objetivos los requerimientos funcionales [Hadad'97]. 
"una excepción provoca una seria interrupción en el escenario ... generalmente refleja el malfuncionamiento of falta de algún recurso" [Leite '97].

\section{solicitud de reserva}

- Goal

- Atender una solicitud de reserva realizada por una persona, agencia u otro hotel.

- Context

- Se realiza en la recepción del Hotel

- Resources

- tarifa

- planilla de reservas

- planilla de ocupación de habitaciones

- Teléfono

- Fax

- e-mail

- Actors

- recepcionista

- agencia

- otro hotel

- pasajero/huésped/pax

- Episodes

- El recepcionista verifica en la planilla de ocupación de habitaciones la disponibilidad de habitaciones y si hubiera, informa la tarifa y solicita la aprobación de la persona, agencia o de otro hotel.

- El recepcionista registra el nombre del pasajero / huésped / pax, cantidad de pasajeros, tipo de habitación, día de ingreso, día de egreso y tarifa en la planilla de reservas

- El recepcionista actualiza la disponibilidad de habitaciones en la planilla de ocupación de habitaciones.

- restriction: No hay disponibilidad de habitaciones

- exception: El teléfono, el fax o el e-mail no funcionan.

Nuevamente aparece el uso de simbolos del LEL y/o escenarios para describir los escenarios, cumpliéndose aquí también el principio de circularidad. (Las palabras que aparecen subrayadas son los enlaces).

Durante el proceso de validación de los escenarios, se generaron varias versiones del conjunto de escenarios que testimonian la evolución del proceso de elicitación a medida que crece la comprensión del macrosistema por parte de los desarrolladores y la idea de lo que se espera de ellos por parte de los clientes. usuarios.

En este punto se pudo observar el uso de distintos verbos con casi idéntico significado, entonces se decidió confeccionar una lista con los verbos más usados y eliminar todos aquéllos que fueran sinónimos para evitar dispersiones en el vocabulario minimo.

Muchas veces ocurre que dos o más escenarios resultan idénticos en todos sus atributos, es decir realizan prácticamente las mismas acciones pero cambia algún detalle y, por supuesto, el nombre, en ese caso se procede a su unificación. En el 
trabajo sobre el Hotel, esto ocurrió con los escenarios Cancelación de la reserva y No show

\title{
Nombre: $\underline{\text { Cancelación de la reserva }}$
}

Objetivo: dar de baja una solicitud de reserva de un pasajero / huésped / pax

Contexto: Se realiza en la Recepción del Hotel. Existe una solicitud de reserva para el pasajero / huésped / pax y el_pasajero / huésped / pax, agencia u otro hotel desea cancelarla. La fecha de solicitud de la cancelación de reserva es anterior al día de ingreso registrado en la solicitud de reserva.

Actores:

\author{
$\underline{\text { Recepcionista }}$ \\ agencia \\ Otro hotel \\ pasajero/huésped/pax
}

Recursos:

Planilla de reservas

Planilla de ocupación de habitaciones.

Teléfono

Fax

e-mail

Set de episodios:

- El Recepcionista recibe el pedido del pasajero / huésped / pax, agencia u otro hotel para la anulación de una solicitud de reserva.

- El Recepcionista elimina la solicitud de reserva de la planilla de reservas.

- El Recepcionista actualiza la disponibilidad de habitaciones en la planilla de ocupación de habitaciones.

Restricciones:

Casos alternativos:

El teléfono, el fax o el e-mail no funcionan.

\section{Nombre: $\underline{\text { No show }}$}

Objetivo: Cancelación de una reserva por no presentación del pasajero / huésped / pax

Contexto: Se realiza en la Recepción del Hotel. Existe una solicitud de reserva y el pasajero / huésped / pax no se presenta.

Actores:

$\underline{\text { Recepcionista }}$

Recursos:

Planilla de reservas

Planilla de ocupación de habitaciones. 
Set de episodios:

- Si el pasajero/huésped/pax no se presenta en el período comprendido entre las 12 hs del día de ingreso establecido en la solicitud de reserva y las 06 hs. del día siguiente entonces el Recepcionista produce la baja de la solicitud de reserva en la planilla de reservas.

- El Recepcionista actualiza la disponibilidad de habitaciones en la planilla de ocupación de habitaciones.

Restricciones:

Casos alternativos:

Estos dos escenarios se unificaron en la versión definitiva hecha con la herramienta $\mathcal{B M W}$ [Antonelli '02]. Al hacerlo, se puede optar por el nombre de uno de los escenarios fusionados o bien proponer uno nuevo.

\section{cancelación de la reserva}

- Goal

- Dar de baja una solicitud de reserva de un pasajero / huésped / pax.

- Context

- Se realiza en la recepción del Hotel. Existe una solicitud de reserva para un pasajero / huésped / pax.

- Resources

- planilla de reservas

- planilla de ocupación de habitaciones

- Teléfono

- Fax

- e-mail

- Actors

- recepcionista

- agencia

- otro hotel

- pasajero/huésped/pax

- Episodes

- if el recepcionista recibe el pedido de anulación de una solicitud de reserva o el pasajero / huésped / pax no se presenta en el período comprendido entre las $12 \mathrm{hs}$ del día de ingreso establecido en la solicitud de reserva y las $06 \mathrm{hs}$. del día siguiente then el recepcionista elimina la solicitud de reserva de la planilla de reservas.

- El recepcionista actualiza la disponibilidad de habitaciones en la planilla de ocupación de habitaciones.

- exception: El teléfono, el fax o el e-mail no funcionan.

Es mucho más fácil para los grupos intervinientes analizar y así poder convalidar o rechazar situaciones que se dan en el ambiente de trabajo en base a una representación de los hechos que simplemente respondiendo a un cuestionario. El hacer aportes e intervenir en el análisis hace que los clientes-usuarios, pero especialmente los usuarios, pierdan un poco la desconfianza o temor ante ese nuevo 
sistema de trabajo y se sientan involucrados en el proyecto, se genera así un ambiente de colaboración más cordial "una consideración importante para eléxito en la interpretación de las necesidades de los usuarios es elcompromiso óptimo de todos los participantes" [Loucopoulos 95]. En este punto influye el aspecto sociológico del proceso de elicitación. 


\section{CAPITULO III}

\section{TEORÍA DE MEDICIONES}

"Aún cuando no es claro cómo medir un atributo, el simple ac to de proponer esas mediciones abrirá un de bate que lleva a una mayor comprensión"

Norman E. Fenton

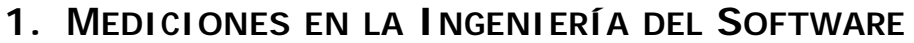

"Para mejorar la rigurosidad de las mediciones en la Ingeniería de Software, no se debe restringir ni el tipo ni el rango de las mediciones que se pueden fracer. Más aún, al medir lo inmedible se mejora la comprensión de ciertos atributos y entidades, convirtiendo a la Ingeniería de Software en una disciplina tan poderosa como las otras ramas de la ingenierí" [Fenton 96].

Lo enunciado en el párrafo anterior constituye un desafío para el ingeniero de Software que se proponga investigar y ahondar en la propuesta de nuevas formas de medir su trabajo con la me ta final puesta en la mejora del producto.

Para optimizar elgerenciamiento de un desarrollo de software, es importante mejorar la habilidad para la identificación, medición y control de los parámetros esenciales que lo afectan y ése es el objetivo de la medición del software [Mills '88].

$S i$ es posible realizar mediciones en las fases tempranas del desarrollo del sistema que permitan conocer características del proceso y asi saber si se está desarrollando un proceso viable, esto significaría un gran aforro de recursos [Hammer '97].

Las métricas del software se aplican tanto al proceso como a los productos de las distintas etapas del proceso, desde los requerimientos hasta el sistema instalado [Mills '8 8 ].

En cualquier actividad de desarrollo de software, según [Fenton'96] existen tres tipos de entidades:

$\checkmark$ Procesos: actividades relacionadas al software

$\checkmark$ Productos: artefactos resultantes de procesos

$\checkmark$ Recursos: entidades necesarias para la realización de procesos

A suvez, estas entidades tienen atributos que pueden ser:

$\checkmark \quad$ Internos: se miden por observación de la entidad, independientemente de su comportamiento

$\checkmark$ Externos: sólo pueden medirse en función de cómo se relaciona la entidad con su ambiente, su comportamiento es más importante que la entidad mis ma. 


\section{DEFI $\mathcal{N} I$ CIÓ $\mathcal{N} \mathcal{D E} \mathcal{M E D I C I O ́} \mathfrak{N}$}

En principio las palabras medida, medición y métrica son sustantivos que pueden considerarse como sinónimos. Sin embargo en este trabajo se las considerará con una semántica levemente diferente.

Se entiende como medida (eninglés: measure) a "un número o simbolo asignado a una entidad como resultado de la caracterización de alguno de sus atributos". Ampliando esta definición, también se puede decir que una medida es una "estimación o evaluación de una cantidad hecha según su relación a otra cantidad de la misma especie tomada como una unidad y como término de comparación" [Sopena '63].

Por otro lado, la palabra métrica (en inglés: metrics) es, según el diccionario [Sopena '63], todo aquello "perteneciente o relativo al metro o medida". Vale aquí tener en cuenta la consideración hecha por [Zuse '95] en relación a la palabra métrica cuando dice que "no se considerará como una medida de espacio, sino como un mapeo o relación entre objetos empiricos y objetos numéricos que preserve todas las estructuras y las relaciones, es decir un flomomorfismo".

En cambio medición (en inglés: measurement) es según [Fenton 96] el "proceso por el cual números o simbolos se asignan a atributos de las entidades en el mundo real de forma tal de poder describirlos acorde a reglas claramente definidas" y según [Sopena '63] "Acción y efecto de medir"

Tomando las consideraciones expuestas por [Zuse 95] que observa elfiecho de que en la literatura los términos métrica y medida se usan como sinónimos y de lOffen 97] que recomienda preferir la palabra medición a métrica ya que la connotación de métrica sugiere una medida de distancia en ciencias como física y matemática, es que en este trabajo se optó por el uso de la palabra medición para significar el resultado de la aplicación de un conjunto de mediciones para cuantificar atributos de ciertos artefactos.

Un conjunto de mediciones serán "aceptables" si facilitan el desarrollo de modelos que sean capaces de predecir parámetros delproceso o producto en estudio, no simplemente que lo describan. Por lo tanto las mediciones ideales, según [Mills '88] de berian ser:

- Simples: el proceso de evaluación debe ser claro y sencillo

- Objetivas: deberán ser inde pendientes de la voluntad del evaluador/medidor

- Fácilmente obtenibles: es decir a un costo razonable

- Válidas: las me didas de be rían me dir lo que dicen medir

- Robustas: relativamente insensibles a pequeños cambios en la entidad que se mida

Un conjunto de mediciones aplicadas a algún artefacto concreto y conocido, permiten cuantificar sus características y medir el tamaño. Intuitivamente el tamaño se reconoce como un concepto importante en elárea de las mediciones [Briand 96].

$\mathcal{E l}$ tamaño de los artefactos es relevante desde la definición de los requerimientos de software hasta el momento de la entrega del artefacto final [Rule '01]. En una etapa posterior, ayudan a hacer inferencias y llegar a generalizaciones que sirvan para todos los productos del mismo tipo. Cualquier artefacto de software está caracterizado por atributos que deben ser estudiados y evaluados, uno de ellos es el tamaño [Morasca '99]. Otros atributos internos son más dificiles de medir 
porque existen diferentes opiniones respecto a lo que significan o a como se miden [Fenton 96], sin embargo, una vez que se logra identificar los atributos que resulten útiles para caracterizar el producto en cuestión y se establezcala forma en que se medirán, el proble ma de la evaluación estará solucionado.

\section{TEORÍA DE LAS MEDICIONES}

Puede suceder que frente a algún atributo se pueda realizar una valoración subjetiva, pero en este caso no se tendrá una medida en el sentido de la teoría de la medición que establece la necesidad de formalizar la noción intuitiva que se tie ne del mundo [Fenton'96].

La teoría de las mediciones establece que algunas propiedades pueden medirse y que debe existir alguna relación entre lo que se puede medir y lo que se desea saber. Algunas veces resulta dificil relacionar lo que es posible medir con algún atributo, por ejemplo se puede contar la cantidad de apóstrofes por página que tiene un texto, pero esto no dice nada acerca de su calidad [Le Vie '00].

Tendemos a comprender la naturaleza de las cosas comparándolas entre si, no asignándole números, eso es una relación e mpírica [Fenton 96]. Cualquier me dición se basa en la ide a de la comparación. Se pue de enunciar la siguiente relación empírica: "el producto $X$ es mejor que el $y$ porque tiene un color más intenso o se degrada más lentamente", pero esta observación está viciada de la subjetividad delobservador. Se buscan métodos de medición que permitan comparar algún atributo de la entidad con un patrón previamente establecido y consensuado que no dependa de apreciaciones personales, es decir que sea objetivo para poder aplicarlo para evaluar el mis mo tipo de artefactos en diferentes circunstancias.

Existen dos caminos para efectuar comparaciones entre entidades del mundo real o dominio:

1. La percepción individual de características fisicas de la entidad o atributos: con este mecanismo puede haber una gama de calificativos tan amplia como la cantidad de personas opinando. Es útil para orientar la búsqueda de atributos medibles y realizar apreciaciones más precisas. Esto da origen a las Mediciones $S$ ubjetivas [Mills '88] que no son mediciones en el estricto significado de lo que se definió como medición, pues dependen de la apreciación personal del observador y son dificilmente reproducibles para diferentes casos. Por ejemplo: la clasificación del sof tware como muy bueno, bueno, regular y malo.

2. la medición previa de algún atributo de la entidad y la posterior asignación de un valor dentro de un rango de valores preestablecidos: números, letras o cualquier conjunto de simbolos que guarden cierta relación entre ellos. Se podrían objetar la exactitud de los instrumentos de medición, la idoneidad de quien los usa o los fundamentos de los cálculos propuestos. Entonces lo que se somete a comparación son las magnitudes que cuantifican la entidad, ahora sí, inobjetables e idénticas para cualquier número de individuos. Cuando se utilizan números, se establece un mapeo matemático que preserva las relaciones observadas en el punto 1. Estas mediciones son llamadas por [Mills '88] Mediciones Objetivas pues con ellas se 
obtendrán valores idénticos para una medida dada independientemente del observador. Por ejemplo: la cantidad de líne as de código.

\section{TEORÍA REPRES EXNACIONAL DE LA MEDICIÓN}

La Teoría Representacional de la Me dición establece que la información que se obtiene como una medida debería representar atributos de la entidad que se observa y la manipulación de esa información de bería, también, preservar las relaciones que se observan entre entidades [Fenton'96].

En general se prefiere cuantificar los atributos antes que cualificarlos pues siempre es más confiable una metodología estricta y repetible al criterio de los investigadores individuales que podrán estar influenciados por cuestiones subje tivas. Aún en este caso se puede emitir un juicio, pero no sería una medida desde el punto de vista de la Teoría de la Medición. Se busca que las bases de medición sean dificilmente objetables y sincomponentes abstractos de forma talque, conociendo el valor del atributo, podamos imaginar alguna característica de la entidad que lo posee y elaborar conclusiones.

Se debe poner énfasis en establecer mediciones de calidad y no mediciones mecánicas. Una medición mecánica de un documento, se basa en una particularidad que puede medirse mecánicamente, por ejemplo: cantidad de palabras por página, cantidad de páginas de un libro, cantidad de palabras en una definición, etc. que aisladas no caracterizan al artefacto que describen, pero que, sin embargo, pueden ser útiles si se utilizan en un esquema comparativo. Una medición de calidad intenta medir la "bondad" de un documento, por ejemplo: cantidad de errores por página, cantidad de palabras normalizadas respecto a la cantidad de palabras en una definición, etc. son magnitudes que dan idea de cualidades que son medibles. Se espera que una medición de calidad sea un predictor de la calidad de un documento. Si no lo es, entonces es una medición mecánica y no tiene mucha utilidad [Le Vie bo].

\section{MEDICIONES INNMEI AT AS Y MEDICIONES CALCULADAS}

Se deben distinguir dos formas de cuantificación:

$\checkmark$ Mediciones: son las que se observan directamente, se efectúan sobre la entidad y asíse obtiene el valor del atributo en forma directa [Fenton 96] y no involucran ningún otro atributo o entidad. También se las llama Mediciones Primitivas [Mills '88] o Mediciones Inmediatas. Por ejemplo: la altura de un edificio o la cantidad de palabras que contie ne un simbolo del LEL.

$\checkmark$ Cálculos: se combinan mediante fórmulas mediciones inmediatas para obtener valores que demuestren las relaciones que existen entre ellas, son las Mediciones Calculadas, también se las conoce como Mediciones Computadas [Mills '88] o Mediciones Indirectas [Fenton 96]. Por ejemplo el rendimiento de un automóvil que relaciona la distancia recorrida con el combustible consumido o la media que relaciona el total de palabras del LEL con la cantidad de entradas que contiene. 
En este punto es importante establecer el papel del análisis estadístico en el ámbito de las mediciones que, según [Fenton 96], permite expresar en forma matemática relaciones entre los valores medidos de algunos atributos, es decir evidencia patrones de comportamiento delconjunto.

Como conclusión se puede afirmar que una medida debe especificar no sólo el dominio y el rango, sino también las reglas que gobiernan el mapeo entre ellos [Fenton $96]$.

6. PROCESO DE MEDICIÓN

La condición de representación establece que si una relación $\mathcal{R}$ mapea entidades con números y relaciones empíricas con relaciones numéricas de forma tal que las relaciones empiricas preserven y sean preservadas por las relaciones numéricas, entonces se podrá definir a esta relación $R$ como una medición para el atributo [Fenton 96]. El mapeo al que se llama medición se lo suele denominar también representación u homomorfismo porque la medición representa el atributo en el mundo numérico.

Los pasos a seguir para determinar si una relación empirica puede ser conside rada una me dición se grafican en la Figura I I I.1
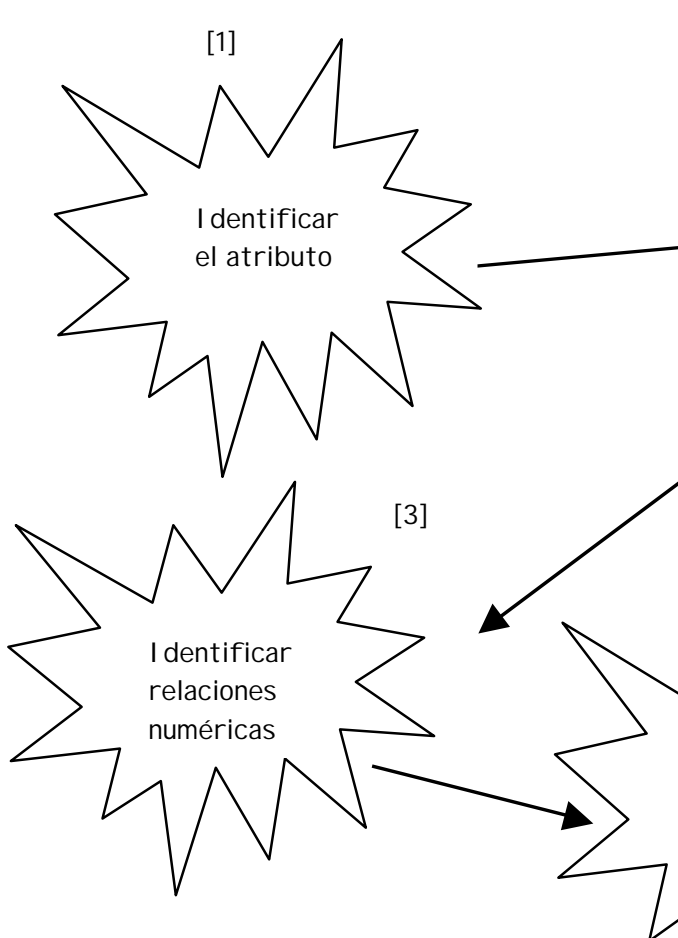

[2]
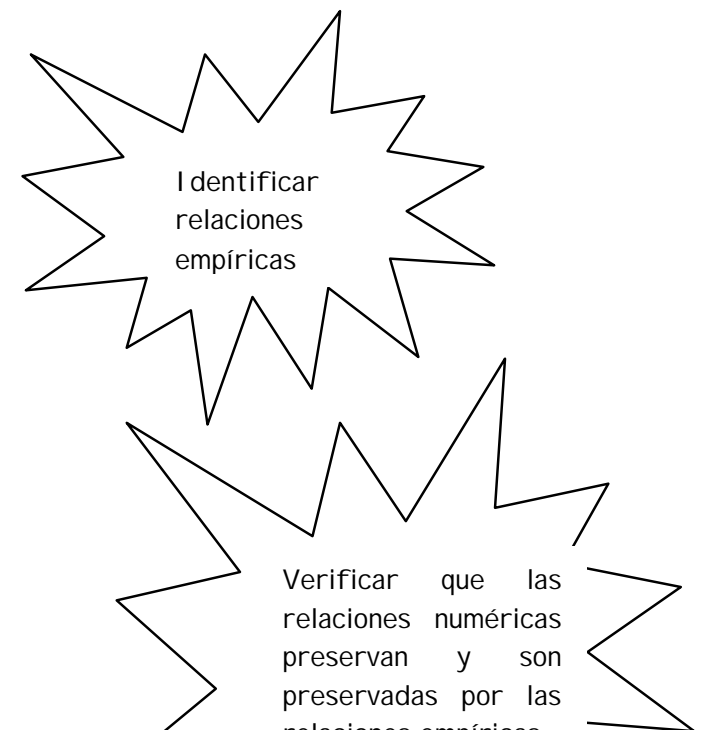
relaciones empíricas

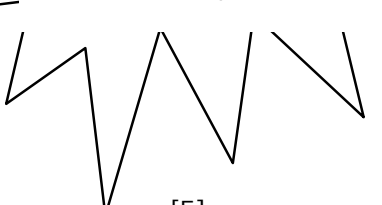

Figura I I I.1: Condición de representación [Fenton 96]

En primer lugar se deben identificar atributos de las entidades que en este caso son: simbolo del $L \mathcal{L E} \mathcal{A}$ y simbolo del $L \mathcal{L E} \mathcal{B}[1]$, por ejemplo establecer el tamaño en función de las palabras que intervienen en su definición. Luego se buscan relaciones 
empiricas para el atributo en cuestión, por ejemplo el simbolo $\mathcal{A}$ tiene un tamaño mayor que el simbolo $\mathcal{B}$ porque la cantidad de palabras de $\mathcal{A}$ es mayor que la cantidad de palabras de $\mathcal{B}$, simbólicamente: $C P(\mathcal{A})>C P(\mathcal{B})[2]$. S i, la cantidad de palabras de $\mathcal{A}$ es $C 1$ y la cantidad de palabras de $\mathcal{B}$ es $C 2$, entonces se tendría que cumplir que $C 1>$ C2 [3]. Al contar las palabras usadas para definir el simbolo, se estará estableciendo un mapeo entre los números y la cantidad de palabras, por ejemplo $C 1=50$ y $C 2=34$ [4]. Se verifica que 50>34, todos los pasos se pueden cumplimentar, por lo tanto se han podido satisfacer las etapas que requiere la condición de representación y se llega así a establecer que la cantidad de palabras de un simbolo del LEL $C P(S n)$ es una medición que representa su tamaño [5].

Para concluir, se tiene:

1. Relación empírica no mapeable: "el simbolo $\mathcal{A}$ está mejor redactado que el simbolo $\mathcal{B}^{\prime \prime}$

2. Relación empirica que se puede mapear: "la cantidad de palabras del simbolo $\mathcal{A}$ es mayor que la cantidad de palabras del simbolo $\mathcal{B}^{\prime \prime}$

3. Medición del atributo usando una escala predefinida: "la cantidad de palabras del simbolo $\mathcal{A}$ es ..." Medición inmediata

4. Medición de un atributo como combinación de otros: "La media de palabras usadas para definir un simbolo es de ..." Medición calculada

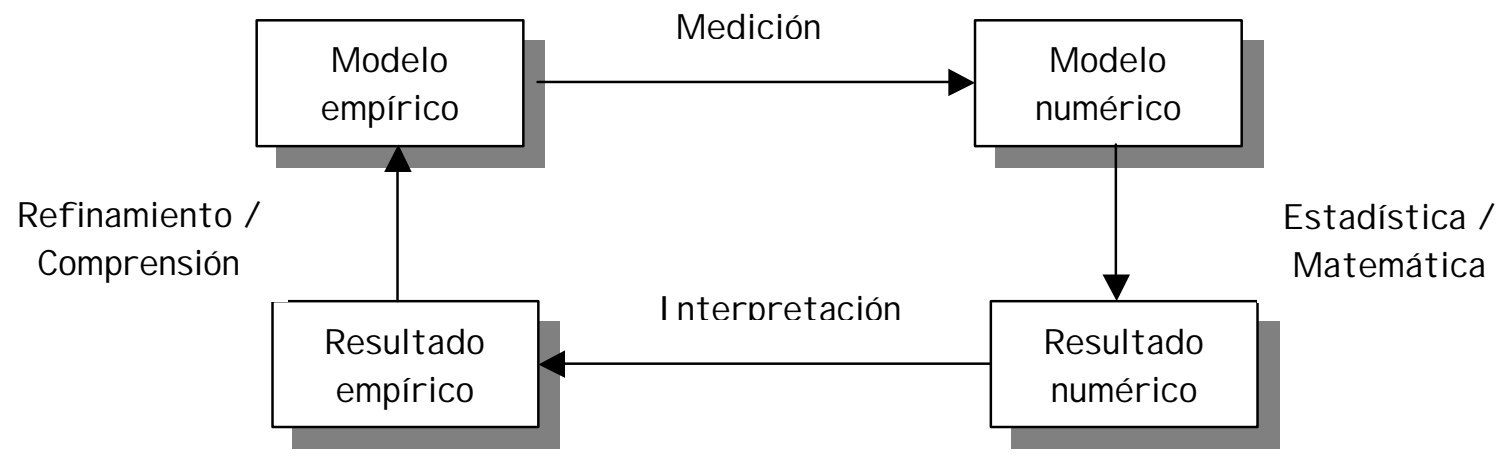

Figura III.2: Relación entre los modelos y sus resultados [Offen'97]

$\mathcal{A l}$ abordar un estudio sobre alguna entidad, es importante poder describir un modelo empirico como una abstracción de la realidad que permita obviar los de talles y ver la entidad o concepto desde una perspectiva particular, en definitiva, el contexto en el que se efectuará la medición a partir del cual se pueda elaborar un modelo numérico que incorpore las mediciones basadas en aspectos bien definidos del modelo empirico, es decir, definir una asociación entre atributos que será el sustento del proceso de medición. Para ello se establecen reglas y excepciones que encuadren el modelo sobre el cual se harán las mediciones contando con el auxilio de la estadística y la matemática se obtienen resultados numéricos. La interpretación de estos datos permite comprender mejor la abstracción de la que se partió produciendo resultados empiricos que sometidos a los refinamientos necesarios permiten llegar a una mejor definición del modelo empírico. Este proceso realiza su propio feedback en ciclos 
sucesivos y puede detenerse cuando el analista considere que hallegado al punto de exactitud que satisfaga las necesidades de la tarea, tal como se indica la Figura II I .2 .

Una ve $z$ que se ha conseguido establecer la naturaleza de las mediciones se debe tener en cuenta que existen dos tipos de datos a los que se pueden aplicar: datos en bruto que son los recolectados en la medición inicial de procesos, productos o recursos y datos refinados que se obtienen después de extraer los elementos esenciales de la información en bruto para que los analistas puedan derivar valores para los atributos. La Figura II I.3 esquematiza este proceso.

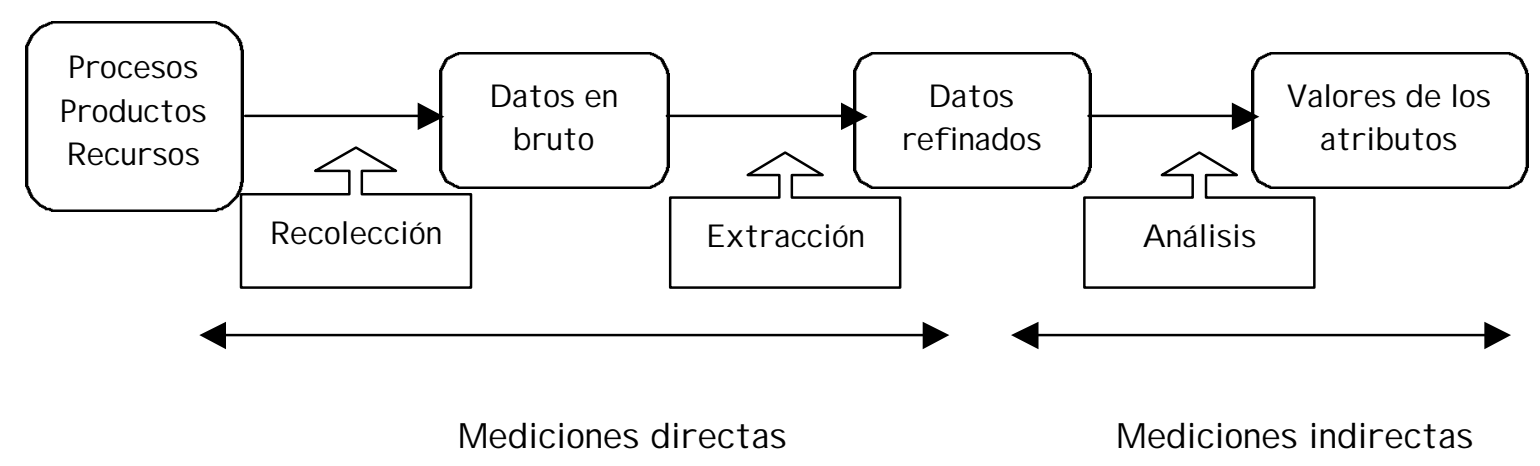

Figura I I I.3: Proceso de me dición [Fenton'96]

En esta tesis se recolectan datos en bruto del producto obtenido del proceso de elicitación de requerimientos realizado utilizando la técnica de LELÉEscenarios. Estos datos son, por ejemplo, cantidad de palabras que contiene una definición del LEL, cantidad de simbolos del LEL que intervienen en la definición de un simbolo del LEL o cantidad de simbolos que contiene un LEL entre otros. $\mathcal{A}$ partir de estos datos se establecen relaciones entre ellos obteniéndose datos refinados como por ejemplo, el cociente entre la cantidad de verbos normalizadas y la cantidad de oraciones utilizadas en la definición de un simbolo del LEL, la tasa de uso de una entrada del LEL, el promedio de algún tipo de palabra utilizada para definir un simbolo del LEL, etc.

Se espera que analizando los datos se puedan establecer valores para, por ejemplo, el atributo tamaño del $L \mathcal{L E}$. 


\section{CAPITULO IV}

\section{LENGUAJE NATURAL}

Cuando se trata de describir situaciones u objetos en lenguaje natural, el analista se enfrenta al hecho de que no todas las personas manejan el mismo vocabulario, dependiendo esto de su nivel socioeconómico, su medio cultural, su especialidad profesional y en gran parte del idioma que se utilice. Sin embargo, si se toman los vocabularios de todos los hablantes de una lengua, siempre existe una intersección entre ellos. De otro modo no podría darse la mutua comprensión, puede decirse que ésta no es total o plena pero no puede decirse que no existe en absoluto.

Existen otros dos conjuntos que tampoco son coextensivos:elde los items que un determinado hablante puede interpretar y elde los que efectivamente usa. Se los pue de llamar, respectivamente, su competencia léxica pasiva y su competencia léxica activa.

$\mathcal{A}$ veces, además, las diferencias entre hablantes son de connotación, (cuando una palabra significa dos ideas, una accesoria y otra principal [Marín 's2]), cosa esperable porque la connotación consiste en los valores asociativos, que pueden ser individuales o de denotación, (es decir que indican, anuncian o significan (Marin 's2]) lo que es más grave, pues conduce a incomprensiones más severas.

Es sorprendente ver que muchas veces se considera que se entendió perfectamente el contenido de un texto, cuando en realidad no es asi. El uso de un lenguaje formal no garantiza ni claridad ni comprensión. Es común que en una charla informal sobre, por ejemplo, cuestiones legales donde alguno de los involucrados es un abogado, el resto de las personas queden con dudas respecto al significado de muchos de los términos por él usados o sencillamente no llegue a comprender completamente el concepto que encierra una palabra de uso común cuando se utiliza en un contexto legal [gackson 95 ].

"... para que la conversación entre cuasi desconocidos sea fructífera necesitamos un orden deldia con asuntos que discutir y un lenguaje comprensible para todos.... El problema reside en encontrar un código de expresión común válido para todos" [Lagunilla 95 ].

El uso del lenguaje natural para describir sistemas que poseen un vocabulario especifico (biología, medicina, informática, religión, etc.) tiene tres problemas graves cuando no todos los interlocutores lo manejan.

"...todos los lenguajes tienen tres problemas: ambigüedad, inexactitud e inconsistencia... a pesar de la severidad de los problemas que heredan los lenguajes naturales, tienen grandes ventajas. Su amplitud de vocabulario y la comprensión de sus reglas sintácticas facilita la comunicación entre los participantes del proyecto" [Wils on '99].

$\checkmark$ Ambigüedad: se produce cuando es posible dar varias y distintas interpretaciones a una palabra que deben ser aclaradas por el contexto a fin de comprender la significación que se le quiere dar. 
Por ejemplo palabras como banco, llama, vela, que tienen más de una posible interpretación, deberán ser resueltas en su ambigüedad, ya que el sentido dado por la expresión en cuestión será o bien uno o bien elotro, no ambos a la vez. Este es un ejemplo de ambigüedad léxica. También hay ejemplos de ambigüedades estructurales: "Los alumnos y las alumnas inteligentes" puede interpretarse de dos maneras diferentes según que el adjetivo "inteligentes" dependa sólo del segundo sustantivo "alumnas" o de la conjunción de los dos sustantivos "alumnos y alumnas". № es en este caso el significado de las palabras el que produce la ambigüedad, sino la configuración sintáctica.

$\checkmark$ Inexactitud o vaguedad: es la imprecisión que proviene de términos cuya denotación es gradual $y$ de límites borrosos. Por ejemplo cuando se dice: "El agua está tibia" no es posible saber cuál es la temperatura a la que el agua posee la condición de estar tibia y para cada individuo esta propiedad hará referencia a una temperatura diferente.

$\checkmark$ Inconsistencia: parte de usar la misma palabra con distinto valor en diferentes pasajes. Por ejemplo: "Camila sueña en el banco que queda frente al banco donde está su madre". Aquí cualquiera de las dos referencias que se hacen a la palabra "banco" puede significar un mueble para sentarse o un lugar donde se realizan transacciones financie ras. La inconsistencia es un proble ma que afecta al procesamiento de los textos (es decir, no a la estructura semántica de las lenguas, pero sía su uso) y en realidad no sería una característica distinta de la ambigüe dad y la inexactitud, que son atributos de las lenguas naturales, sino más bien, una consecuencia de ellas

Muchas palabras o frases tienen significados que dependen exclusivamente del contexto en el que se usan [Wilson '97]. Esto es una característica del lenguaje natural: nos remite a un contexto para encontrar su intencionalidad.

$\mathcal{A}$ estos problemas no escapa el proceso de elicitación de requerimientos dado que en su génesis intervienen tanto desarrolladores como usuarios y clientes, cada uno con sus necesidades y términos propios. En las entrevistas se deben evitar términos vagos para referirse, por ejemplo, a cantidades o tamaños: mucho, grande, rápido, etc; como así también comparaciones: similar, dentro del mismo rango, casi como, etc. Hay palabras que tienen un significado impreciso y que por lo tanto convie ne evitar: fácil, normal, adecuado, efectivo, etc.

Tampoco es recomendable el uso de palabras o frases que den libertad para interpretar su implementación, por ejemplo: si es apropiado, si se requiere, si resulta practicable, casisiempre, en la mayoría de los casos, habitualmente, etc.

En cualquier definición expresada en lenguaje natural, es importante controlar la cantidad de palabras que se usarán y el esque ma que se seguirá para estructurarla. Los conceptos que tienen una estructura previamente detallada son más sencillos de construir, de purar y comprender. $\mathcal{E l} L \mathcal{L E}$ al ser un conjunto de definiciones no escapa a estas conside raciones.

El hecho de usar lenguaje natural para describir situaciones mediante el LEL $y$ los Escenarios, ayuda a la validación con el cliente - usuario y cumple con la misión de mejorar la comunicación entre los desarrolladores [Leite '00]. Sin embargo, luego de analizar varios LEL para aplicarle posteriormente las mediciones propuestas, se ha 
visto que, en pos de obtener mejores resultados, fue conveniente realizar algunas correcciones a las definiciones del $\mathcal{L E L}$, como por ejemplo si la definición incluye el nombre de la entrada que está definiendo, és ta se reemplazó por un sinónimo.

$\mathcal{E L} L \mathcal{L} L$ es una representación en lenguaje natural del lenguaje usado en la aplicación y ayuda a capturar su vocabulario [Leite '00] y a establecer un código de expresión común válido para todos [Lagunilla 95] los intervinientes en el proceso de elicitación, evitando que el ingeniero de requerimientos introduzca su propio vocabulario. 


\section{CAPITULO V}

\section{PALABRA NORMALIZADA}

Frente al desafío de realizar mediciones sobre un texto, el principal obstáculo con el que se encuentra quien decida realizar esta tarea será la riqueza del lenguaje natural que lo hace tan diverso y complejo como cada autor.

En esta tesis se parte de la base de establecer:

a) Definición de palabra normalizada: consideraciones a tener en cuenta para aceptar una palabra como parte constitutiva de la definición de un símbolo del LEL, es decir los vocablos se considerarán palabras normalizadas en función de su peso semántico para transmitir un concepto.

Por ejemplo hay grupos de vocablos que podrían eliminarse sin que por ello pierda significado la definición que los utiliza y otros que son imprescindibles.

Ejemplo: $a=$ "Es la solicitud de un servicio de reparaciones"

$a^{\prime}=$ "Solicitud de reparaciones"

b) Determinación del tamaño de un símbolo del LEL: cantidad de palabras normalizadas que intervienen en su definición.

Este capítulo se dedicará a enunciar pautas para determinar las palabras normalizadas, es decir se ocupará del punto a). El capítulo VI será el que explique las definiciones necesarias para realizar mediciones que permitan establecer el tamaño del símbolo del LEL, es decir tratará el punto b).

\section{DEFINICIÓN DE PALABRA NORMALIZADA}

Se propone redefinir el concepto de palabra en esta tesis con el objeto de contar sólo las palabras que sean "imprescindibles" para transmitir la idea a la que hace referencia el símbolo pudiendo así apartarse de las singularidades del analista que realiza el trabajo.

Se llamará Palabra normalizada a aquella palabra que si se eliminara de la definición de la entrada ésta perdería sentido. Se obtendrán aplicando las reglas de normalización, que se especificarán más adelante. Este conjunto de palabras jerarquizadas, que denominamos normalizadas, formarán parte del subconjunto de palabras que al contarlas darán una medida del tamaño del símbolo.

\section{TÉCNICA PROPUESTA PARA APLICAR LAS REGLAS DE NORMALIZACION}

Los documentos expresados en lenguaje natural responden a la estructura (llamada también sintaxis o gramática) del idioma en el que fueron escritos, esta estructura resulta demasiado compleja y la cantidad de términos involucrados es muy 
grande cuando el objetivo es aplicar algoritmos matemáticos, es por ello que se debe buscar una forma alternativa de representar el texto que se desea procesar.

En la literatura sobre recuperación de información (Information Retrieval) para aplicar los algoritmos de "clustering" o agrupamiento a los documentos, éstos se representan con un vector t-dimensional, donde $t$ es la cantidad de conceptos permitidos que quedan después de aplicar al documento original técnicas para disminuir la cantidad de palabras que se usarán para caracterizarlo y así solucionar el problema de la maldición de la dimensionalidad "the curse of dimensionality" [Zervas '00]. La ausencia de un término se indica con 0 o -1 la presencia de un término se indica con 10 un número positivo que indica su peso [Faloutsos '95]. En [Jones '95] se utiliza el mismo concepto pero se establece un vector binario para cada documento, donde si el elemento i-ésimo está presente se indica con 1 y con 0 si no lo está.

Teniendo en cuenta esta forma de representación de documentos, se aplicará una técnica similar para representar cada una de las entradas del LEL, entonces se tendrá que:

Dada una entrada $x$ del LEL se construye para cada oración $v$, un vector binario $v^{\star}$ en el que cada posición equivale a una palabra de la definición y cuya dimensión será la cantidad de palabras de $v, C P(v)$.

Por ejemplo, si se considera la oración siguiente:

$v=$ "La Administración la utitiza para el calculo de la facturación"

al contar la cantidad de palabras se obtendrá $C P(v)=10$, por lo tanto el vector $v^{\star}$ contará con diez posiciones, ocupadas, en principio por unos.

$$
v^{*}=\left(\begin{array}{llllllllll}
1 & 1 & 1 & 1 & 1 & 1 & 1 & 1 & 1 & 1
\end{array}\right)
$$

A medida que se le aplique al vector $v^{\star}$ las reglas de normalización propuestas habrá algunas posiciones que cambiarán el 1 por 0 si la palabra representada no se considera normalizada y por lo tanto no se cuenta y otras que quedarán en 1.

Se define el operador $\Psi$ que aplica sucesivamente y sin un orden específico todas las reglas de normalización al vector $v^{\star}$ transformando 1 en 0 en los casos en que detecte palabras que no sean normalizadas, obteniéndose el vector $v^{\star}$ transformado:

$$
\Psi\left(v^{\star}\right)=\operatorname{Rk}\left(\ldots\left(\operatorname{R2}\left(\operatorname{R1}\left(v^{\star}\right)\right)\right) \ldots\right)=\left(\begin{array}{llllllllll}
0 & 1 & 0 & 1 & 0 & 0 & 1 & 0 & 0 & 1
\end{array}\right)
$$

\section{REGLAS PARA DEFINIR UNA PALABRA NORMALIZADA}

En el área de la recuperación de la información (Information Retrieval), se utilizan diversas técnicas para reducir la dimensión de los documentos y representarlos sólo con las palabras más significativas.

Una de estas técnicas es la remoción de palabras de alta frecuencia y bajo contenido semántico [Van Rijsbergen '79]. Para ello se utilizan "stoplists" o listas de palabras consideradas poco relevantes para el contexto que se está analizando. Se ha 
visto en la práctica que estas palabras coinciden con artículos, preposiciones, conjunciones, etc [Faloutsos '95].

La estrategia que se usará en este trabajo consiste en definir reglas para cada clase ${ }^{1}$ de palabra. La aplicación de estas reglas a cada una de las palabras que conforman una entrada del LEL permiten determinar si son normalizadas o no. Si lo fueran, formarán parte del subconjunto de palabras que se tendrán en cuenta al realizar mediciones, caso contrario no se considerarán.

Las reglas están numeradas del 1 al 10 y cada una tiene el siguiente formato:

\section{Regla $\mathrm{k}$}

Alcance: especifica la clase de palabra a la que se aplica la regla

Acción: indica si corresponde contar o no la palabra como normalizada

Razón: motivo por el que se aplica la regla

Excepción: si la hubiere, indica la situación en la que la regla no se aplica

Ejemplo: ilustra la aplicación de la regla

A continuación, se proponen las reglas que permitan eliminar aquéllas palabras que no sean consideradas normalizadas.

\section{Regla 1}

Alcance [artículos y palabras coordinativas (preposiciones y conjunciones)]

Acción [no se cuentan]

Razón [no tienen peso a la hora de definir un concepto]

Excepción [cuando los artículos en una oración cumplen la función de un sustantivo se cuentan]

Ejemplo:

$a=$ "La Administración la utitiza para el calculo de la facturación"

A partir de la oración a, se construye el vector $a^{\star}$ cuya dimensión es $C P(a)=10$

$$
a^{*}=\left(\begin{array}{llllllllll}
1 & 1 & 1 & 1 & 1 & 1 & 1 & 1 & 1 & 1
\end{array}\right)
$$

La aplicación de la Regla 1 al vector $a^{*}$, lleva a eliminar las palabras la y el que son artículos y para y de que son preposiciones, esto queda indicado colocando 0 en las posiciones pertenecientes a las palabras eliminadas. Aquí se aplica la excepción que indica la Regla 1 a la posición 3 que contiene el artículo la cumpliendo la función de un sustantivo. Entonces se obtiene el vector:

$$
R 1\left(a^{\star}\right)=\left(\begin{array}{llllllllll}
0 & 1 & 1 & 1 & 0 & 0 & 1 & 0 & 0 & 1
\end{array}\right)
$$

\footnotetext{
${ }^{1}$ El Apéndice A explica las características de cada una de las clases de palabras usadas.
} 


\section{Regla 2}
Alcance [pronombres]
Acción [no se cuentan]
Razón [son palabras no descriptivas]

Excepción [ los pronombres demostrativos esto, éste, aquél y sus formas masculina, femenina y los correspondientes plurales cuando llevan acento están usados como sustantivos o adjetivos,

Ejemplo: entonces se cuentan]

$$
b=\text { "En aquellos casos donde se necesite su conocimiento" }
$$

El vector correspondiente a la oración b es:

$$
b^{\star}=\left(\begin{array}{llllllll}
1 & 1 & 1 & 1 & 1 & 1 & 1 & 1
\end{array}\right)
$$

Al aplicar la Regla 2 al vector $b^{\star}$ se cambian por 0 las posiciones 2,4 y 7 correspondientes a las palabras aquellos, dondey su que son pronombres.

$$
R 2\left(b^{*}\right)=\left(\begin{array}{llllllll}
1 & 0 & 1 & 0 & 1 & 1 & 0 & 1
\end{array}\right)
$$

Los verbos se consideran palabras normalizadas por su carácter central en cualquier oración, sin embargo, cuando se trata de medir el tamaño de una definición se deben establecer ciertas consideraciones con alguno verbos.

\section{Regla 3}

Alcance[formas personales y reflexivas]

Acción [se cuentan como una sola palabra]

Razón[tienen peso semántico sólo si se los considera en conjunto]

Ejemplo [ se convierte, ha guardado, fue comprado]

$$
f=\text { "Se recibe el pago del pasajero/huésped / pax" }
$$

$$
f^{*}=\left(\begin{array}{llllllll}
1 & 1 & 1 & 1 & 1 & 1 & 1 & 1
\end{array}\right)
$$

Al aplicársele la Regla 3 el vector f* se modifica cambiando 1 por 0 en la posición 1 correspondiente a la palabra se, indicando esta acción que en el caso de formas reflexivas se considera una sola palabra normalizada.

El vector obtenido luego de aplicarle la Regla 3 al vector $f^{\star}$ es:

$$
R 3\left(f^{\star}\right)=\left(\begin{array}{llllllll}
0 & 1 & 1 & 1 & 1 & 1 & 1 & 1
\end{array}\right)
$$




\section{Regla 4}

Alcance [verbos vacíos o livianos (que necesitan de otras palabras para definir su semántica)]

Acción [se cuentan como una sola palabra]

Razón [tienen peso semántico sólo si se los considera en conjunto]

Ejemplo [tuvo sueño, dio consuelo, se puede realizar, debe llenar, trata de comprender, pasa a ser, lleva a cabo]

$g=$ "Puede hacer un voucher para el pasajero / huésped/pax"

La oración g está representada por el vector binario $g^{*}$ de dimensión $C P(g)=9$

$$
g^{\star}=\left(\begin{array}{lllllllll}
1 & 1 & 1 & 1 & 1 & 1 & 1 & 1 & 1
\end{array}\right)
$$

La Regla 4 indica en su enunciado que los verbos vacíos se contarán como una sola palabra en conjunto con las otras palabras que necesita para definir su semántica, en este ejemplo puede hacer se cuenta como una palabra y no como dos, entonces se cambiará por 0 sólo el elemento que se encuentra en la posición 2 del vector $g^{\star}$ y que corresponde a la palabra hacer.

$$
R 4\left(g^{\star}\right)=\left(\begin{array}{lllllllll}
1 & 0 & 1 & 1 & 1 & 1 & 1 & 1 & 1
\end{array}\right)
$$

\section{Regla 5}

Alcance [verbo sery estar (cuando se puede reemplazar por ser)]

Acción [no se cuentan]

Razón [son prescindibles en una oración que expresa una definición, son verbos funcionales]

Ejemplo:

$h=$ "Es la solicitud de un servicio de reparaciones"

$$
h^{*}=\left(\begin{array}{llllllll}
1 & 1 & 1 & 1 & 1 & 1 & 1 & 1
\end{array}\right)
$$

$R 5\left(h^{\star}\right)=\left(\begin{array}{llllllll}0 & 1 & 1 & 1 & 1 & 1 & 1 & 1\end{array}\right)$

Al aplicar la transformación que indica la Regla 5 al vector $h^{*}$, se obtiene el vector $\mathrm{R} 5\left(h^{\star}\right)$ donde se ha eliminado el verbo es que ocupa la primera posición.

\section{Regla 6}

Alcance [conjunto de palabras que forman un símbolo del LEL]

Acción [se cuentan como una sola palabra]

Razón [expresan un concepto]

Ejemplo [Planilla de ocupación de habitaciones, fichero de pasajeros, pasajero / huésped / pax] 
$\mathrm{j}=$ "Puede hacer una solicitud de reserva o una cancelación de reserva para el pasajero/huésped /pax"

A continuación se escribe el vector que representa la oración j de dimensión $C P(j)=$ 16

$$
j^{*}=\left(\begin{array}{llllllllllllllll}
1 & 1 & 1 & 1 & 1 & 1 & 1 & 1 & 1 & 1 & 1 & 1 & 1 & 1 & 1 & 1
\end{array}\right)
$$

La aplicación de la Regla 6 tiene como consecuencia cambiar por ceros todas las posiciones pertenecientes a las palabras que forman una entrada del LEL menos una. Por una cuestión práctica, se dejará en 1 sólo la correspondiente a la primera posición de la entrada y el resto de las posiciones se cambiarán por 0 , como consecuencia cada entrada del LEL se contará como una única palabra normalizada, independientemente de la cantidad de palabras que la compongan.

$$
R 6\left(j^{\star}\right)=\left(\begin{array}{llllllllllllllll}
1 & 1 & 1 & 1 & 0 & 0 & 1 & 1 & 1 & 0 & 0 & 1 & 1 & 1 & 0 & 0
\end{array}\right)
$$

En el vector $j^{*}$ de este ejemplo se cambian por 0 las posiciones 5 y 6 correspondientes a las palabras de reserva, las posiciones 10 y 11 correspondientes a las palabras de reservay las posiciones 15 y 16 que representan las palabras huésped y pax. Con lo cual se asegura que cada entrada del LEL se contará como una sola palabra normalizada.

\section{Regla 7}

Alcance [conjunto de palabras que designan un elemento del UdD o una frase de uso común]

Acción [se cuentan como una sola palabra]

Razón [expresan un concepto]

Ejemplo [control remoto TV, servicios turísticos, punto de vista, tarjeta magnética, teniendo en cuenta]

$\mathrm{k}=$ "Se recibe la tarjeta codificada, el control remoto TV, La llave del minibar y de la caja de seguridad si estuviera en poder del pasajero/huésped/pax"

$$
k^{*}=\left(\begin{array}{lllllllllllllllllllllllllll}
1 & 1 & 1 & 1 & 1 & 1 & 1 & 1 & 1 & 1 & 1 & 1 & 1 & 1 & 1 & 1 & 1 & 1 & 1 & 1 & 1 & 1 & 1 & 1 & 1 & 1 & 1
\end{array}\right)
$$

El vector $k^{*}$ representa la oración del ejemplo; luego de aplicarle la Regla 7 , se cambian por 0 las posiciones 8 y 9 correspondientes a las palabras remoto TV y 18 y 19 que corresponden a de seguridad. Entonces se obtiene:

$$
R 7\left(k^{\star}\right)=\left(\begin{array}{lllllllllllllllllllllllllll}
1 & 1 & 1 & 1 & 1 & 1 & 1 & 0 & 0 & 1 & 1 & 1 & 1 & 1 & 1 & 1 & 1 & 0 & 0 & 1 & 1 & 1 & 1 & 1 & 1 & 1 & 1
\end{array}\right)
$$




\section{Regla 8}

Alcance [caracteres especiales y números]

Acción [se cuentan como una sola palabra]

Razón [se consideran en conjunto con los otros caracteres que acompañan pues describen un único objeto]

Ejemplo [4598, \$135, malenac@gmx.net, \#25]

\section{Regla 9}

Alcance [abreviaturas que acompañan a otras palabras]

Acción [se cuentan como una sola palabra]

Razón [expresan un concepto]

Ejemplo [10 km, 22:30 hrs., Sr. Ramírez]

\section{$\underline{\text { Regla } 10}$}

Alcance [frases y estructuras]

Acción [se cuenta la estructura completa como una palabra]

Razón [existen indicadores individuales que se repiten a lo largo del texto [Wilson '97]]

Ejemplo [estructura si... entonces]

$m$ = "Sú el recepcionista recibióel voucher entonces extiende la factura"

El ejemplo usa un caso típico en el que muchas veces puede faltar la palabra entonces, aún cuando la frase la incluya en forma tácita. El vector $m$ * será:

$$
m^{*}=\left(\begin{array}{llllllllll}
1 & 1 & 1 & 1 & 1 & 1 & 1 & 1 & 1 & 1
\end{array}\right)
$$

que al aplicarle la Regla 10 quedará como:

$$
\mathrm{R} 10\left(m^{*}\right)=\left(\begin{array}{llllllllll}
1 & 1 & 1 & 1 & 1 & 1 & 0 & 1 & 1 & 1
\end{array}\right)
$$

Estas reglas nunca superponen su alcance, por lo tanto son complementarias, como consecuencia de esta característica resulta indistinto el orden en el que se apliquen. 


\section{MEDICIONES APLICADAS A LAS PALABRAS NORMALIZADAS DEL SIIMBOLO}

Las primeras mediciones que surgen al enfrentarse con un documento son las referidas a la cantidad de palabras usadas o a algún tipo particular de palabras que resulte relevante para el contexto que se trata de analizar. En general, palabras que permitan hacer inferencias respecto al tamaño del texto y por qué no, a su complejidad, si de ello se pudiera deducir, obteniéndose así las denominadas mediciones inmediatas.

Posteriormente al relacionar estas mediciones en fórmulas se obtendrán resultados que permitirán establecer otras características del documento y se las denominará mediciones calculadas.

Se tratará de describir una entidad, en este caso el símbolo del LEL, a través de algunos de sus atributos que pueden ser representados por números [Fenton '96].

Las mediciones que se proponen en este capítulo son atómicas en el sentido que se refieren exclusivamente a los símbolos del LEL individualmente considerados. Algunas de ellas son:

$>$ Cantidad de palabras que contiene la definición

$>$ Cantidad de verbos que contiene la definición

$>$ Cantidad de otros símbolos del LEL que contiene la definición

$>$ Cantidad de veces que se usa un símbolo dado del LEL en la definición de otros símbolos del LEL

$>$ Cantidad de oraciones que contiene la definición

Es muy difícil establecer una medición rigurosa de tamaño cuando se trata de lenguaje natural debido a que existen tantos estilos retóricos como individuos, lo que lleva a expresar la misma idea con palabras y construcciones lingüísticas muy diversas.

Sin embargo, el lenguaje usado para las definiciones, tanto en un diccionario como en el LEL trata, en general, de ser preciso y conciso. Por lo tanto se puede considerar que no varía demasiado la cantidad de palabras usadas. Esto sugiere que la "medición ingenua" que representa contar la cantidad de palabras, sería efectivamente una medida a tener en cuenta para reflejar la magnitud de una definición.

Para ejemplificar las definiciones que se establecen en este capítulo, se usará el símbolo del LEL "Hotel" fichero de pasajeros. 


\section{fichero de pasajeros}

- Noción

$a=$ "Contiene el conjunto de planilla del pasajero"

b = "Se guarda en un mueble de archivo en la recepción"

- Impacto

$$
\begin{aligned}
c= & \text { "Se utitiza como archivo histórico para el check in a un pasajerol } \\
& \text { huésped / pax." }
\end{aligned}
$$

\section{MEDICIONES INMEDIATAS}

Estas mediciones surgen del simple hecho de contar los elementos constitutivos de una definición sin tener en cuenta las características de esos elementos.

Se puede pensar que un primer refinamiento para calcular el tamaño de una definición podría ser no contar aquéllas palabras que estuvieran repetidas. Sin embargo, a medida que se avance en la lectura de esta tesis, se verá que la determinación del tamaño tiene una relación directa con la necesidad de utilizar una clase de palabra para transmitir un concepto y no con la cantidad de veces que un mismo vocablo se encuentre en la definición de un símbolo del LEL.

Definición 1: dada una entrada $x$ de un LEL, se indica con $C P(x)$ la cantidad de palabras que intervienen en su definición, incluyendo todas las ocurrencias de una misma palabra.

Contando las palabras de cada una de las oraciones que intervienen en la definición fichero de pasajeros, se obtendrá un total de 31 palabras, es decir:

$$
C P(\text { fichero de pasajeros })=C P(a)+C P(b)+C P(c)=31
$$

Las palabras Noción e Impacto no se incluyen en la cantidad de palabras y tampoco las palabras que forman el nombre de la entrada pues no participan en la definición.

Esta definición simplemente cuenta todas las palabras que intervienen en la definición sin reparar en sus características o en la cantidad de veces que pudieran estar repetidas.

Definición 2: dada una entrada $x$ de un LEL, se indica con $C O(x)$ la cantidad de oraciones que se usaron para definir el símbolo $x$.

La cantidad de oraciones usadas en una definición, al igual que las palabras y su cantidad, están fuertemente influenciadas por el estilo retórico de la persona que realiza la redacción. 
Para definir el símbolo fichero de pasajeros se utilizaron tres oraciones. Entonces se obtiene:

$$
C O(\text { fichero de pasajeros })=3
$$

Con las definiciones dadas hasta ahora, se podría pensar que queda cubierto el tema de la determinación del tamaño de una entrada.

Ahora bien, si se le pidiera a otra persona que definiera el objeto fichero de pasajeros seguramente hubiera utilizado otras palabras y una cantidad diferente de palabras, entonces es obvio que resultan ambiguas para determinar el tamaño de un símbolo pues dependen fuertemente del estilo del redactor. Esto lleva a pensar que el simple hecho de contar palabras $u$ oraciones no es suficiente para determinar el tamaño de una definición.

En este punto es conveniente dar mayores precisiones respecto a lo que se entiende por tamaño de un símbolo del LEL en el contexto de esta tesis. Como una primera aproximación, se considerará al tamaño como la cantidad de palabras o de alguna clase de palabras que sean imprescindibles (en contraposición a superfluas o redundantes) para explicar el significado de la definición del símbolo del LEL en el que intervienen.

Tratándose de Lenguaje Natural existen otras consideraciones a tener en cuenta, consideraciones vinculadas a la semántica, no ya de cada palabra sino de las frases o conjunto de palabras que expresan una idea, un concepto. Surge el problema de evaluar una disciplina tan rica como la expresión de ideas con herramientas de extrema rigidez como son las matemáticas. Entonces se debe apelar a consideraciones que permitan establecer un mapeo válido entre ambas disciplinas, se llega así al concepto de Palabra Normalizada1, que permitirá acotar la cantidad de palabras que se cuentan considerando su valor semántico para describir un símbolo del LEL.

\subsection{MEDICIONES APLICADAS A PALABRAS NORMALIZADAS}

Definición 3: una palabra $p$ perteneciente a una entrada $x$ de un $L E L$, se denominará palabra normalizada si luego de aplicarle las reglas de normalización, no ha sido eliminada.

Las siguientes definiciones establecen las pautas para construir el subconjunto de palabras normalizadas.

Definición 4: dada una entrada $x$ de un LEL, $x^{*}$ representa el vector de dimensión $C P(x)$ que tiene un 1 en cada posición y se indicará como $R_{k}\left(x^{*}\right)$ al vector resultante de aplicar la regla k-ésima al vector $x^{*}$ que transforma 1 en o si la palabra no es normalizada

Es claro que $x^{\star}$ y $R_{k}\left(x^{\star}\right)$ tienen la misma dimensión.

${ }^{1}$ Para ampliar este tema, ver el Capítulo V 
Nótese que la aplicación de las reglas en ningún momento sugiere que la definición deba ser escrita nuevamente obviando las palabras no normalizadas, por el contrario, la idea es establecer el tamaño de una definición en función de las palabras relevantes que se usaron en ella sin alterar su formato original.

Definición 5: dada una entrada $x$ de un LEL se indica con CPN $(x)$ la cantidad de palabras normalizadas que son las que cumplen con la Definición 3 y que intervienen en su definición.

Para facilitar el proceso de análisis se escribe un vector por cada oración de la entrada fichero de pasajeros, entonces se tendrá que:

$$
\begin{aligned}
& a^{*}=\left(\begin{array}{lllllll}
1 & 1 & 1 & 1 & 1 & 1 & 1
\end{array}\right) \\
& b^{\star}=\left(\begin{array}{llllllllll}
1 & 1 & 1 & 1 & 1 & 1 & 1 & 1 & 1 & 1
\end{array}\right) \\
& c^{*}=\left(\begin{array}{llllllllllllll}
1 & 1 & 1 & 1 & 1 & 1 & 1 & 1 & 1 & 1 & 1 & 1 & 1 & 1
\end{array}\right)
\end{aligned}
$$

aplicando todas las reglas de normalización propuestas, se llega a los siguientes vectores transformados:

$$
\begin{aligned}
& \Psi_{k=1}^{10}\left(a^{\star}\right)=\left(\begin{array}{lllllll}
1 & 0 & 1 & 0 & 1 & 0 & 0
\end{array}\right) \begin{array}{l}
\begin{array}{l}
\text { se eliminaron las palabras el, de y del pasajero, } \\
\text { entonces } \operatorname{CPN}(a)=3
\end{array}
\end{array} \\
& \Psi_{k=1}^{10}\left(b^{\star}\right)=\left(\begin{array}{llllllllll}
0 & 1 & 0 & 0 & 1 & 0 & 0 & 0 & 0 & 1
\end{array}\right) \begin{array}{l}
\text { se eliminaron las palabras se, en, un, de } \\
\text { archivo, en y la, entonces } C P N(b)=3
\end{array} \\
& \Psi_{\mathrm{k}=1}^{10}\left(c^{*}\right)=\left(\begin{array}{llllllllllllll}
0 & 1 & 0 & 1 & 1 & 0 & 0 & 1 & 0 & 0 & 0 & 1 & 0 & 0
\end{array}\right) \begin{array}{l}
\text { se eliminaron las palabras se, } \\
\text { como, para, el, in, a, un, }
\end{array}
\end{aligned}
$$

Finalmente, al sumar las componentes de los tres vectores, se llega a:

$$
C P N(\text { fichero de pasajeros })=C P N(a)+C P N(b)+C P N(c)=11
$$

Para obtener este valor, se contabilizan todas las palabras que no se eliminaron al aplicárseles las reglas propuestas para considerar una palabra como normalizada, es decir la cantidad de palabras normalizadas de la entrada fichero de pasajeros será la suma de los elementos de los vectores que la representan.

El proceso utilizado se puede generalizar diciendo:

Dado el símbolo $x$ del LEL cuyo vector binario es $x^{\star}=\left(x_{1}, x_{2}, \ldots, x_{i}\right)$ al aplicarle la transformación $\Psi$, se obtendrá el vector $\Psi\left(x^{\star}\right)=\left(x_{1}^{\Psi}, x_{2}^{\Psi}, \ldots, x_{i}^{\Psi}\right)$. La sumatoria de los elementos de $\Psi\left(x^{*}\right)$ dará la cantidad de palabras normalizadas de la definición. 
Esto se puede expresar como: $C P N(x)=\sum_{i=1}^{C P(x)} x_{i}^{\Psi}$

siendo $x_{i}^{\Psi}$ la componente i-ésima del vector $x^{\star}$ al que se le aplicaron las reglas de transformación.

Definición 6: dada una entrada $x$ de un LEL, se indica con CS(x) la cantidad de símbolos del LEL que intervienen en su definición.

La cantidad de símbolos que usa una definición $x, \operatorname{CS}(x)$ es una medida de la utilización de los símbolos y por lo tanto de la aplicación del principio de circularidad [Leite '96].

Los símbolos del LEL utilizados en la definición de fichero de pasajeros son: planilla del pasajero, recepción, check in, pasajero/huésped/pax. Por lo tanto se tendrá:

$$
\operatorname{CS}(\text { fichero de pasajeros })=4
$$

Definición 7: dada una entrada $x$ de un LEL, se indica con CVN $(x)$ la cantidad de verbos normalizados que intervienen en la definición de la entrada y que cumplen con la Definición 3.

Para aplicar el proceso de normalización a la entrada fichero de pasajeros se toman las siguientes consideraciones:

Contiene: forma conjugada de un verbo, se cuenta como palabra normalizada.

Se guarda: forma personal, según la Regla 3 es una sola palabra normalizada, entonces se pone a 0 la posición correspondiente a la palabra se.

Se utiliza: ídem al anterior.

Entonces se llega a:

$$
C V N(\text { fichero de pasajeros })=3
$$

Se observa que la cantidad de palabras normalizadas incluye los verbos normalizados y los símbolos del LEL, pero la suma de estas dos cantidades no es igual a la cantidad de palabras normalizadas que contiene una definición, es decir:

$$
\operatorname{CPN}(x) \geq\{\operatorname{CVN}(x)+\operatorname{CS}(x)\}
$$

entonces se concluye que existen otras palabras normalizadas que no son ni verbos $\mathrm{ni}$ símbolos del LEL.

Definición 8: dada una entrada $x$ de un LEL, se indica con COPN $(x)$ la cantidad de otras palabras normalizadas que no son símbolos del LEL ni verbos normalizados.

Esta magnitud se puede obtener aplicando la siguiente fórmula en lugar de contar: 


$$
\operatorname{COPN}(x)=\operatorname{CPN}(x)-\operatorname{CVN}(x)-\operatorname{CS}(x)
$$

Tomando como ejemplo la entrada fichero de pasajeros, se obtiene:

COPN(fichero de pasajeros) $=$ CPN(fichero de pasajeros) $-C V N($ fichero de pasajeros) $-C S$ (fichero de pasajeros) $=12-3-4=5$

\section{MEDICIONES CALCULADAS}

En lo que sigue se propondrán otras mediciones que se expresan en función de las mediciones ya propuestas.

Las mediciones calculadas surgen de la aplicación de fórmulas en las que se combinan mediciones inmediatas y otros parámetros que puedan caracterizar al modelo en estudio.

Definición 9: dada una entrada $x$ de un LEL se indica con $\operatorname{COER}(x)$ al coeficiente de redundancia que es el cociente entre la cantidad de palabras normalizadas y la cantidad de palabras utilizadas en la definición de un símbolo $x$ del LEL.

Para efectuar el cálculo se usará la ecuación:

$$
\operatorname{COER}(x)=\operatorname{CPN}(x) / C P(x)
$$

Esta medición cuantifica la cantidad de palabras superfluas o innecesarias que contiene la definición de un símbolo del LEL.

Obsérvese que dada la naturaleza del símbolo del LEL el $\operatorname{COER}(x)$ es siempre estrictamente menor que 1 pues en toda definición hay palabras que no se consideran normalizadas pero que son necesarias para la redacción y no para la semántica. Por otro lado, el $\operatorname{COER}(x)$ no debería ser 0 ya que esto indicaría la ausencia de palabras normalizadas en la definición, por lo tanto se espera que sea estrictamente mayor que 0 .

Entonces se podría decir que el $\operatorname{COER}(x)$ es una medida de la calidad de una definición.

Remitiéndose nuevamente a la entrada fichero de pasajeros, se obtiene:

COER(fichero de pasajeros) $=$ CPN (fichero de pasajeros) / CP (fichero de pasajeros) $=12 / 31=0,39$

Este resultado evidencia una definición factible de ser mejorada utilizando en su redacción menor cantidad de palabras no normalizadas.

Definición 10: dada una entrada $x$ de un LEL se indica con COES $(x)$ al coeficiente de simplicidad que es el cociente entre la cantidad de verbos 
normalizadas y la cantidad de oraciones utilizadas en la definición del símbolo.

La fórmula que permite realizar este cálculo es:

$$
\operatorname{COES}(x)=\operatorname{CVN}(x) / \operatorname{CO}(x)
$$

$\operatorname{COES}(x)\left\{\begin{array}{cl}>1 & \text { existen oraciones con más de un verbo normalizado } \\ =1 & \text { cada oración contiene un verbo normalizado } \\ <1 & \text { existen oraciones sin verbos normalizados }\end{array}\right.$

En el caso de la entrada fichero de pasajeros se obtiene:

COES(fichero de pasajeros) $=$ CVN(fichero de pasajeros $) / C O$ (fichero de pasajeros $)$ $=3 / 3=1$

Aparentemente en este caso se da la relación más favorable, observando la entrada se concluye que efectivamente cada oración contiene un verbo normalizado.

Puede suceder que la definición de un símbolo incluya tres oraciones, una de ellas utilice dos verbos normalizados, otra un verbo normalizado y la otra ninguno, el resultado del cálculo del $\operatorname{COES}(x)$ no reflejará este hecho y será aceptable, por lo tanto, este coeficiente no se debe interpretar como un indicador absoluto sino simplemente un llamado de atención en los casos en los que el valor se aleje notoriamente de 1.

El carácter central del verbo determina su correlación con el número de cláusulas de un discurso que será igual al número de verbos. Es deseable un COES igual a 1 porque indica que la oración tiene un solo verbo. Son oraciones simples que es lo que se busca para tener un LEL bien construido. Sin embargo, hay excepciones:

a) se ejecutan varias acciones sobre el mismo sujeto, entonces la oración tiene varios verbos y esto evita la repetición del sujeto en distintas oraciones.

b) una misma acción se puede realizar sobre varios sujetos distintos, así se construye una oración que si bien no es simple, cumple con la premisa de usar un verbo por oración.

Definición 11: dada una entrada $x_{i}$ de un LEL se indica con FI $\left(x_{i}\right)$ y se denomina Fan In a la cantidad de todos los símbolos que se usan para definir el símbolo $x_{i}$.

Se construye para el LEL una matriz de adyacencia ponderada ${ }^{2} \mathrm{~F}$ de dimensión $\operatorname{CSL}(L) \times C S L(L)$, siendo CSL la cantidad total de símbolos del LEL $L$, donde a partir de la suma de los elementos de la fila i-ésima se obtiene el Fan In de la entrada $x_{i}$ con la ecuación:

${ }^{2}$ Ver apartado 4 en este capítulo 


$$
\mathrm{FI}\left(\mathrm{x}_{\mathrm{i}}\right)=\sum_{\mathrm{j}=1}^{\operatorname{CSL}(L)} \mathrm{Fij}
$$

siendo Fij la cantidad de veces que el símbolo $x_{j}$ se usa en la definición del símbolo $x_{i}$ Siguiendo con la entrada fichero de pasajeros, se calcula un valor:

$$
F I(\text { fichero de pasajeros })=4
$$

Este valor de Fan In se obtiene sumando las columnas de la fila correspondiente a la entrada fichero de pasajeros en la matriz de adyacencia ponderada del LEL "Hotel"

Un símbolo con un $F I\left(x_{i}\right)$ alto podría indicar que es un concepto complejo, en cuya definición intervienen muchos símbolos del LEL y sugeriría un buen uso del principio de vocabulario mínimo.

El $F I\left(x_{i}\right)$ fue definido como $C S(x)$ en la Definición 6. En ella se estableció que el valor del $\operatorname{CS}(x)$ se puede obtener contando directamente las entradas que se encuentran en la definición de $x$, incluyendo las repeticiones que pudiera haber.

Definición 12: dada una entrada $x_{j}$ de un LEL se indica con $F O\left(x_{j}\right)$ y se denomina Fan Out a la cantidad de veces que el símbolo $x_{j}$ es usado en la definición de otros símbolos.

Se construye para el LEL una matriz de adyacencia ponderada $F$ de dimensión $\operatorname{CSL}(L) \times C S L(L)$, siendo CSL la cantidad total de símbolos del LEL $L$, donde a partir de la suma de los elementos de todas las filas de la columna $j$ se obtiene el Fan Out de la entrada $x_{j}$.

$$
F O\left(x_{j}\right)=\sum_{i=1}^{C S(L)} F i j
$$

siendo Fij la cantidad de veces que el símbolo $x_{i}$ usa en su definición al símbolo $x_{j}$

El $F O\left(x_{j}\right)$ permite calcular la cantidad de veces que un símbolo es usado para definir otros símbolos del LEL. Si la matriz de adyacencia es ponderada, lo que se obtiene es la cantidad de veces que el símbolo es utilizado por otros símbolos con todas sus repeticiones. En este trabajo se especificó que se tendrían en cuenta todas las ocurrencias de las palabras normalizadas, como es de esperar los símbolos del LEL, por el simple hecho de ser las definiciones del LEL, son todos palabras normalizadas y por lo tanto interesa conocer la magnitud incluyendo todas sus ocurrencias.

Para la entrada fichero de pasajeros, se obtiene:

$$
F O(\text { fichero de pasajeros })=4
$$

Este valor de Fan Out se obtiene sumando las filas de la columna correspondiente a la entrada fichero de pasajeros en la matriz de adyacencia ponderada del LEL "Hotel" 3

${ }^{3}$ Apéndice F Tabla 3 
Se observa que el Fan Out es una medida del símbolo en relación al conjunto de símbolos que lo usan.

Un $F O\left(x_{j}\right)$ alto sugiere que el símbolo $x_{j}$ es muy usado en la definición de otros símbolos del LEL y por lo tanto que éste es un concepto importante. Por otro lado también sugiere un buen uso del principio de vocabulario mínimo.

En un grupo de mediciones, se puede determinar si un valor obtenido es alto o bajo sólo si se lo relaciona con el resto de los componentes del grupo, es por ello que la determinación de los valores extremos se dejará para cuando se realice el análisis del LEL en su conjunto.

Definición 13: dada una entrada $x$ de un LEL se denomina FOI $(x)$ y se calcula como la razón entre el Fan Out y el Fan In para el símbolo $x$ del LEL a la relación entre la cantidad de veces que un símbolo $x$ es usado en las definiciones de otros símbolos y la cantidad de símbolos que usa en su propia definición.

Según la definición, la ecuación para calcular este coeficiente es:

$$
F O I(x)=F O(x) / F I(x)
$$

Tomando como ejemplo la entrada fichero de pasajeros, se obtiene:

FOI (fichero de pasajeros) $=F O($ fichero de pasajeros $) / F I($ fichero de pasajeros $)=$ $4 / 4=1$

Este resultado indica que el símbolo se usa para la definición de otros símbolos la misma cantidad de veces que los símbolos que usa en su propia definición.

Definición 14: dado un LEL Ly una entrada x se indica con TU(x) la tasa de uso de la entrada $x$ y se calcula como la relación entre el Fan Out de la entrada $x$ y la cantidad de entradas que se definieron para el LEL $L$.

Esta relación quedará definida por:

$$
T U(x)=F O(x) / \operatorname{CSL}(L)
$$

siendo CSL(L) la cantidad total de símbolos del LEL L

Para la entrada fichero de pasajeros del LEL "Hotel", se tendrá:

$T U($ fichero de pasajeros $)=F O($ fichero de pasajeros $) / \operatorname{CSL}($ Hotel $)=$

$$
4 / 39=0,10
$$


Valores altos de TU(x) indican entradas del LEL que son muy usadas en la definición de otras entradas. Nuevamente, no se puede determinar a priori qué valores son altos, esto sólo se podrá hacer dentro del contexto de cada LEL.

Esta es una medida del símbolo en relación al conjunto.

\section{APLICACIÓN de LAS REgLAS de NORMALIZACION Y MEDICIONES PROPUESTAS} "Hotel"

Se aplicarán las reglas y definiciones propuestas a dos entradas del LEL

Para facilitar la tarea de la medición de cada entrada se aplicarán las reglas a cada oración y luego se realizará la suma de los valores obtenidos para obtener las mediciones sobre el símbolo.

\section{Ejemplo 1}

\section{Voucher}

- Noción

$a=$ "Contiene los datos del pasajero / huésped / pax, el dia de ingresoy el dia de egreso, la cantidad de pasajeros, nombre y dirección dei Hotel"

$\mathrm{b}=$ "Loprovee la agencia"

$c=$ "Se almacena en la carpeta de la habitación"

- Impacto

$d$ = "El pasajero / huésped / pax, paga el importe del alojamiento en la agencia y éste se acredita en una cuenta corriente del Hotel"

$e=$ "El recepcionista lo recibe del pasajero / huésped / pax"

$f$ = "Se utitiza para cancelar el importe del item correspondiente al atoiamiento en la facturación"

Se establece para la oración a el vector binario $a^{\star}$ cuya dimensión es $C P(a)=$ 24

$$
a^{\star}=\left(\begin{array}{llllllllllllllllllllllll}
1 & 1 & 1 & 1 & 1 & 1 & 1 & 1 & 1 & 1 & 1 & 1 & 1 & 1 & 1 & 1 & 1 & 1 & 1 & 1 & 1 & 1 & 1 & 1
\end{array}\right)
$$

Aplicando las reglas propuestas se eliminan las palabras los, del, huésped, pax, el, de ingreso, el, de egreso, la, de, y y del, que corresponden a las posiciones 2, 4, 6 , $7,8,10,11,12,14,15,16,18,21$ y 23 respectivamente. Entonces el vector $a^{*}$ transformado quedará como:

$$
\Psi_{\mathrm{k}=1}^{10}\left(a^{\star}\right)=\left(\begin{array}{llllllllllllllllllllllll}
1 & 0 & 1 & 0 & 1 & 0 & 0 & 0 & 1 & 0 & 0 & 0 & 1 & 0 & 0 & 0 & 1 & 0 & 1 & 1 & 0 & 1 & 0 & 1
\end{array}\right)
$$


Las mediciones que se obtienen para la oración a son:
$C P(a)=24$
$\operatorname{CPN}(a)=10$
$\operatorname{CVN}(a)=1$
$\operatorname{CS}(a)=3$

Ahora se aplica el mismo proceso para la oración b. El vector binario correspondiente es $b^{\star} y$ tiene 4 posiciones:

$b^{\star}=\left(\begin{array}{llll}1 & 1 & 1 & 1\end{array}\right)$

La aplicación de las reglas al vector b* lleva a:

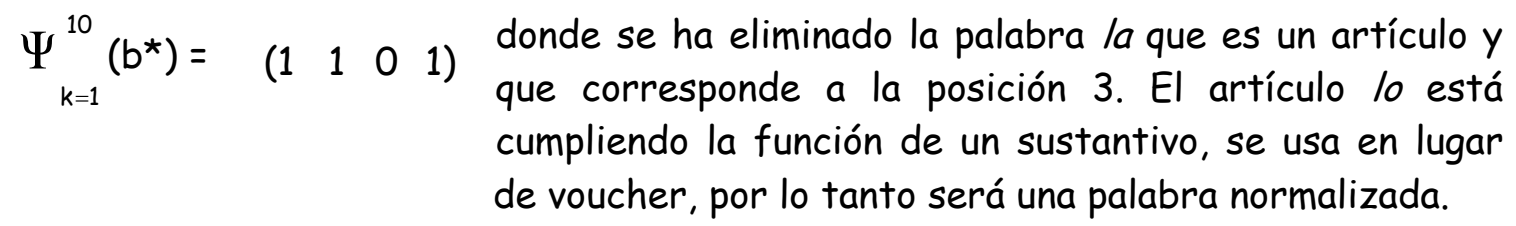
$C P(b)=4$
$C P N(b)=3$
$C V N(b)=1$
$C S(b)=1$

Para la oración c se tendrá:

$$
c^{*}=\left(\begin{array}{llllllll}
1 & 1 & 1 & 1 & 1 & 1 & 1 & 1
\end{array}\right)
$$

y se llega al vector transformado donde se eliminaron las palabras se al aplicar la $\Psi_{k=1}^{10}\left(c^{\star}\right)=\left(\begin{array}{llllllll}0 & 1 & 0 & 0 & 1 & 0 & 0 & 0\end{array}\right) \begin{aligned} & \text { Regla } 3 \text {, en } y \\ & \text { por la Regla } 6\end{aligned}$
$C P(c)=8$
$\operatorname{CPN}(c)=2$
$\operatorname{CVN}(c)=1$
$\operatorname{CS}(c)=1$

La oración d perteneciente al Impacto tendrá un vector de 22 posiciones

$$
d^{\star}=\left(\begin{array}{llllllllllllllllllllll}
1 & 1 & 1 & 1 & 1 & 1 & 1 & 1 & 1 & 1 & 1 & 1 & 1 & 1 & 1 & 1 & 1 & 1 & 1 & 1 & 1 & 1
\end{array}\right)
$$

Las reglas aplicadas a esta oración eliminan las palabras el, huésped, pax, del, en, la, $y$, se, una, corriente en todas las ocurrencias que hubiere. La palabra éste es un pronombre y según la Regla 2 debería ser eliminada pero como está acentuada cumple la función de un sustantivo, entonces no se elimina.

$$
\begin{aligned}
& \Psi_{k=1}^{10}\left(d^{\star}\right)=\left(\begin{array}{llllllllllllllllllllll}
0 & 1 & 0 & 0 & 1 & 0 & 1 & 0 & 1 & 0 & 0 & 1 & 0 & 1 & 0 & 1 & 0 & 0 & 1 & 0 & 0 & 1
\end{array}\right) \\
& C P(d)=22 \\
& C V N(d)=2
\end{aligned}
$$


La oración e tiene 8 palabras y su vector binario es:

$$
\begin{array}{r}
e^{\star}=\left(\begin{array}{llllllll}
1 & 1 & 1 & 1 & 1 & 1 & 1 & 1
\end{array}\right) \\
\Psi_{k=1}^{10}\left(e^{\star}\right)=\left(\begin{array}{llllllll}
0 & 1 & 1 & 1 & 0 & 1 & 0 & 0
\end{array}\right)
\end{array}
$$

se eliminan las palabras el y del que son artículos por aplicación de la Regla 1. El artículo lo no se elimina pues está cumpliendo la función de un sustantivo al reemplazar a voucher.
$C P(e)=8$
$\operatorname{CPN}(e)=4$
$\operatorname{CVN}(e)=1$
$\operatorname{CS}(e)=2$

Finalmente la oración f tiene el siguiente vector:

$$
f^{*}=\left(\begin{array}{llllllllllllll}
1 & 1 & 1 & 1 & 1 & 1 & 1 & 1 & 1 & 1 & 1 & 1 & 1 & 1
\end{array}\right)
$$

luego de aplicar las reglas se eliminan las palabras se, para, el, del, al, en y la quedando el vector transformado como:

$$
\begin{aligned}
& \Psi_{k=1}^{10}\left(f^{\star}\right)=\left(\begin{array}{llllllllllllll}
0 & 1 & 0 & 1 & 0 & 1 & 0 & 1 & 1 & 0 & 1 & 0 & 0 & 1
\end{array}\right) \\
& C P(f)=14 \\
& C V N(f)=1
\end{aligned}
$$

Ahora bien, como ya se dijo, la suma de los valores hallados para cada oración, permite establecer las mediciones para la entrada completa, entonces se tendrá:

$$
\begin{aligned}
& \operatorname{CO}(\text { voucher })=6 \\
& C P(\text { voucher })=\sum_{i=1}^{C O(\text { voucher })} C P\left(o_{i}\right)=24+4+8+22+8+14=80 \\
& \text { CPN(voucher })=\sum_{i=1}^{C O(\text { voucher })} \operatorname{CPN}\left(0_{i}\right)=10+3+2+9+4+7=35 \\
& \operatorname{CVN}(\text { voucher })=\sum_{i=1}^{C O(\text { voucher })} \operatorname{CVN}\left(o_{i}\right)=1+1+1+2+1+1=7 \\
& \operatorname{CS}(\text { voucher })=\sum_{i=1}^{C O(\text { voucher })} \operatorname{CS}\left(o_{i}\right)=3+1+1+2+2+1=10 \\
& \text { COPN(voucher) }=35-7-10=18 \\
& \operatorname{COER} \text { (voucher) }=35 / 80=0,44 \\
& \operatorname{COES} \text { (voucher) }=7 / 6=1,17
\end{aligned}
$$




\section{Ejemplo 2}

\section{Agencia / agencia de viajes}

- Noción

$\mathrm{u}$ = "Es una organización externa e independiente del Hotel"

$w$ = "Administra las ventas de servicios turisticos al pasajero / huésped/ pax"

- Impacto

y = "Puede hacer una solicitud de reserva o una cancelación de reserva para el pasajero/huésped / pax"

$z=$ "Puede hacer un voucher para el pasajero/huésped/pax"

Se establecen los vectores binarios $u^{*}, w^{*}, y^{*} y z^{*}$ que representan las palabras de cada una de las oraciones que componen la entrada a analizar y cuyas dimensiones son respectivamente $C P(u), C P(w), C P(y)$ y $C P(z)$.

$$
\begin{aligned}
& u^{\star}=\left(\begin{array}{lllllllll}
1 & 1 & 1 & 1 & 1 & 1 & 1 & 1
\end{array}\right) \\
& w^{\star}=\left(\begin{array}{llllllllll}
1 & 1 & 1 & 1 & 1 & 1 & 1 & 1 & 1 & 1
\end{array}\right) \\
& y^{\star}=\left(\begin{array}{llllllllllllllll}
1 & 1 & 1 & 1 & 1 & 1 & 1 & 1 & 1 & 1 & 1 & 1 & 1 & 1 & 1 & 1
\end{array}\right) \\
& z^{*}=\left(\begin{array}{llllllllll}
1 & 1 & 1 & 1 & 1 & 1 & 1 & 1 & 1
\end{array}\right)
\end{aligned}
$$

Después de aplicarle a cada vector las transformaciones indicadas por las reglas propuestas se obtiene:

$$
\begin{aligned}
& \Psi_{k=1}^{10}\left(u^{\star}\right)=\left(\begin{array}{lllllllll}
0 & 0 & 1 & 1 & 0 & 1 & 0 & 1
\end{array}\right) \begin{array}{l}
\text { las palabras que se eliminan para obtener este } \\
\text { vector son: es, una, ey del. }
\end{array} \\
& \Psi_{k=1}^{10}\left(w^{\star}\right)=\left(\begin{array}{lllllllllll}
1 & 0 & 1 & 0 & 1 & 0 & 0 & 1 & 0 & 0
\end{array}\right) \begin{array}{l}
\text { aquí las palabras no normalizadas son: las, } \\
\text { de, } a, l o s, \text { huéspedy pax. }
\end{array} \\
& \Psi_{\mathrm{k}=1}^{10}\left(y^{\star}\right)=\left(\begin{array}{llllllllllllllll}
1 & 0 & 0 & 1 & 0 & 0 & 0 & 0 & 1 & 0 & 0 & 0 & 0 & 1 & 0 & 0
\end{array}\right) \begin{array}{l}
\text { en este vector se } \\
\text { eliminaron las palabras } \\
\text { hacer, una, de, reserva, }
\end{array}
\end{aligned}
$$

0 , una, de, reserva, para, el, huéspedy pax, en total son 12 palabras no normalizadas

$$
\Psi_{\mathrm{k}=1}^{10}\left(z^{*}\right)=\left(\begin{array}{lllllllll}
1 & 0 & 0 & 1 & 0 & 0 & 1 & 0 & 0
\end{array}\right) \begin{aligned}
& \text { las palabras hacer, un, para, el, huésped } y \\
& \text { pax se eliminaron del vector } z^{*}
\end{aligned}
$$


$\operatorname{CO}($ Agencia / agencia de viajes $)=4$

$C P($ Agencia / agencia de viajes $)=\sum_{i=1}^{C O(\text { Agencia })} C P\left(O_{i}\right)=43$

$C P N($ Agencia / agencia de viajes $)=\sum_{i=1}^{C O(\text { Agencia })} C P N\left(o_{i}\right)=15$

$C V N($ Agencia / agencia de viajes $)=\sum_{i=1}^{C O(\text { Agencia })} \operatorname{CVN}\left(\mathrm{o}_{i}\right)=3$

$\operatorname{CS}($ Agencia / agencia de viajes $)=\sum_{i=1}^{\operatorname{CO}(\text { Agencia })} \operatorname{CS}\left(\mathrm{o}_{i}\right)=6$

$\operatorname{COPN}($ Agencia / agencia de viajes $)=15-3-6=6$

$\operatorname{COER}($ Agencia $/$ agencia de viajes $)=15 / 43=0,35$

COES(Agencia / agencia de viajes) $=3 / 4=0,75$

Observando los valores obtenidos para estos ejemplos ninguno es suficiente por sí mismo para dar idea del tamaño del símbolo, entonces se podría pensar que lo que se llama "tamaño del símbolo" sea en realidad algún tipo de relación entre las medidas propuestas.

$$
\operatorname{Tamaño}(x) \approx f(\operatorname{CPN}(x), \operatorname{CS}(x), \ldots, \operatorname{CVN}(x))
$$

\section{COMENTARIOS SOBRE FAN IN Y FAN OUT}

Los conceptos de Fan In y Fan Out aplicados al LEL surgen a partir de los principios del vocabulario mínimo y circularidad.

En la teoría de grafos se llama matriz de adyacencia a una matriz cuadrada en la que tanto filas como columnas representan los vértices o nodos de un grafo. Se selecciona un mismo orden arbitrario para los vértices que se asignan a las filas $y$ columnas de una matriz. Si la intersección de una fila con una columna contiene un uno, significa que esos vértices comparten una arista y cero en caso contrario [Johnsonbaugh '88].

Dado un $L E L=\left\{S_{1}, S_{2}, S_{3}, \ldots, S_{n}\right\}$ se puede completar una matriz de adyacencia $F$ de $n \times n$, teniendo en cuenta que:

entonces:

$$
F_{i j}= \begin{cases}1 & \text { si } S_{i} \text { usa } a S_{j} \\ 0 & \text { en caso contrario }\end{cases}
$$

Fan Out de $S_{j}=F O\left(S_{j}\right)=\sum_{i=1}^{n} F i j \quad$ (sumando filas)

Fan In de $S_{i}=F I\left(S_{i}\right)=\sum_{j=1}^{n} F i j \quad$ (sumando columnas)

Ejemplo: Se tiene un LEL con cuatro símbolos, a partir de la observación de cada uno, se especifica qué entradas usa en su definición sin contar las repeticiones: 


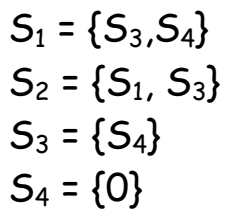

Teniendo en cuenta la definición dada para la matriz de adyacencia con los datos anteriores se puede generar la siguiente matriz para el LEL en estudio:

\begin{tabular}{|l|l|l|l|l|}
\hline & 1 & 2 & 3 & 4 \\
\hline 1 & 0 & 0 & 1 & 1 \\
\hline 2 & 1 & 0 & 1 & 0 \\
\hline 3 & 0 & 0 & 0 & 1 \\
\hline 4 & 0 & 0 & 0 & 0 \\
\hline
\end{tabular}

Al aplicar las definiciones dadas, se obtienen los siguientes valores:

$\begin{array}{ll}\mathrm{FI}\left(S_{1}\right)=2 & \mathrm{FO}\left(S_{1}\right)=1 \\ \operatorname{FI}\left(S_{2}\right)=2 & \mathrm{FO}\left(S_{2}\right)=0 \\ \operatorname{FI}\left(S_{3}\right)=1 & \mathrm{FO}\left(S_{3}\right)=2 \\ \operatorname{FI}\left(S_{4}\right)=0 & \mathrm{FO}\left(S_{4}\right)=2\end{array}$

A partir de contabilizar manualmente el Fan In para cada entrada del LEL y volcarlo por filas en una matriz de adyacencia, se puede obtener el Fan Out de todas las entradas que conforman el LEL aplicando la fórmula dada.

Dentro del contexto del LEL, se construye una matriz de adyacencia ponderada en la que tanto filas como columnas representan las entradas del LEL, en la intersección de la fila con la columna pertenecientes a la misma entrada habrá un 0 , pues una entrada del LEL no debe usarse a sí misma para definirse. Se ingresa por las filas y cuando un símbolo $S_{i}$ utiliza un símbolo $S_{j}$ (que está como columna) en su definición entonces en esa intersección irá el número correspondiente a la cantidad de veces que $S_{j}$ se halla en la definición de $S_{i}$, por eso se dice que la matriz es ponderada o tiene peso. El resto de la matriz se completa con ceros, indicando así que $S_{j}$ no se encuentra en la definición de $S_{i}$.

\section{DESCRIPCIÓN DE LA TABLA 1 DEL APÉNDICE F}

Los cálculos efectuados a cada símbolo del LEL se presentan en la Tabla 1 de los Apéndices F, G y H para los LEL de tres casos de estudio. Tablas como la de la Figura VI.1 se generaron utilizando la planilla de cálculo Excel 2000 de Microsoft. En ella se consignan CP, CPN, CVN, CS, COPN y CO que son el resultado de la contabilidad manual efectuada a cada entrada, el porcentaje resultante de relacionar $C P N$ con $C P$, el resto de los porcentajes en función de CPN y el cálculo de los coeficientes propuestos. 


\begin{tabular}{|c|c|c|c|c|c|c|c|c|c|c|c|c|c|c|c|}
\hline ENTRADAS & $C P$ & & $\bar{N}$ & & $\mathrm{VN}$ & $c$ & s & $\mathrm{CO}$ & $P \mathrm{PN}_{\mathrm{i}}$ & COER & $\cos$ & LOES & $F O$ & FOI! & TU \\
\hline & & & $\%$ & & $\%$ ! & & $\%$ & & $\%$ & & & & & & \\
\hline ADMINISTRACION & 31 & 17 & 55 & 3 & 18 & 2 & 12 & 12 & 71 & 1,82 & 4 & $0,75 !$ & 12 & 6,00 & 3,58 \\
\hline AGENCIA & 43 & 15 & 35 & 3 & $20 ?$ & 6 & $40^{\circ}$ & 6 & $40:$ & 2,87 & 4 & 0,75 & 5 & 0,83 & 1,49 \\
\hline AVISO DE MENSAJE & 40 & 19 & 48 & 5 & 26 & 5 & 26 & 9 & 47 & 2.11 & 4 & 1,25 & 2 & $0,40:$ & 0,60 \\
\hline C. C. CAFETERIA Y/O RESTAURANT & 57 & 26 & 46 & 6 & 23 & 8 & 31 & 12 & $46:$ & 2,19 & $6 !$ & 1,00 & 4 & $0,50 !$ & 1,19 \\
\hline C. C. DEL MINIBAR & 49 & 22 & 45 & 5 & 23 & 8 & $36:$ & 9 & 41 & 2,23 & 6 & 0.83 & 4 & 0,50 & 1,19 \\
\hline C. GASTOS DE LAVANDERIA & 44 & 20 & 45 & 5 & 25 & 7 & 35 & 8 & $40^{\circ}$ & 2,20 & 5 & $1,00 !$ & 4 & 0,57 & 1,19 \\
\hline C. LLAMADOS TELEFONICOS & 37 & 14 & 38 & 4 & $29 !$ & 3 & 21 & 7 & $50:$ & 2,64 & 4 & 1,00 & 3 & 1,00 & 0,90 \\
\hline CANCELACION DE RESERVA & 61 & 17 & 28 & 4 & 24 & 10 & $59:$ & 3 & $18:$ & 3,59 & 5 & 0,80 & 5 & $0,50:$ & 1,49 \\
\hline CARPETA DE LA HABITACION & 47 & 16 & 34 & 3 & 19 ? & 8 & 50 & 5 & 31 & 2.94 & 3 & & 9 & 1,13 & 2,69 \\
\hline
\end{tabular}

Figura VI.1: Tabla 1 Apéndice F: Mediciones aplicadas al símbolo

En la Figura VI.2 se explicitan las fórmulas y funciones de la planilla de cálculo que se utilizaron para calcular los coeficientes.

\begin{tabular}{|c|c|c|c|c|c|c|c|c|c|c|c|c|c|c|}
\hline ENTR & $C P$ & $\mathrm{PN}$ & $\mathrm{VN}$ & CS & $P N:$ & COER & $\mathrm{CO}$ & & COES & FO & & FOI & & TU \\
\hline & i & $\% 1$ & $\% 1$ & $\% 1$ & $\%$ i & & I & & & i & 1 & & 1 & \\
\hline ADMI & 31 i 17 & $55\lceil 3$ & $18 \mathbf{i}$ & $12 i 12$ & $71 \mathbf{i}=$ & $D 74 / C 74$ & 4 & & $\mathrm{~F} 74 /$ M 7 & 7412 & & $574 / \mathrm{H7}$ & $4 \mid=$ & $074 / 3$ \\
\hline AGE & 43115 & $35 ! 3$ & $20 ! 6$ & 4016 & 401 & 0,35 & 4 & I & 0.75 & 15 & i & 0.83 & & 0,13 \\
\hline AVIS & $40 ! 19$ & $48 ! 5$ & $26 ! 5$ & 2619 & $47 !$ & 0.48 & 4 & I & 1,25 & 2 & 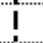 & 40 & $\mathbf{I}$ & 0,05 \\
\hline C. C. CAFETERIA Y/O RESTAURANT & $57 ! 20$ & $46 ! 6$ & $23 ! 8$ & $31 ! 12$ & $46 !$ & 0.46 & 6 & & 1,00 & 4 & ! & 0,50 & & 0,10 \\
\hline 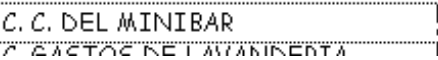 & $\begin{array}{l}49 ! 2 \\
11:\end{array}$ & $\begin{array}{l}45 ! 5 \\
85 ! 5\end{array}$ & & $\begin{array}{l:l}36 ! & 9 \\
35 & \end{array}$ & $\begin{array}{l}41 ! \\
4 \\
\end{array}$ & & 6 & & 1 & \begin{tabular}{l}
4 \\
4 \\
\hdashline
\end{tabular} & & ก" & & 0 \\
\hline
\end{tabular}

Figura VI.2: Fórmulas y funciones de la planilla de cálculo utilizadas 
CAPITULO VII

\title{
MEDICIONES APLICADAS A LAS PALABRAS NORMALIZADAS DEL LEL
}

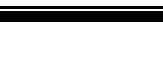

Lo que se denomina Léxico Extendido del Lenguaje (LEL) es un conjunto de definiciones que caracterizan el sistema en estudio.

$\mathcal{T}$ niendo en cuenta las consideraciones fechas en el Capitulo VI "Mediciones aplicadas al simbolo del $\mathcal{L E L}^{\prime}$ sobre la relevancia de las mediciones propuestas para el simbolo, en este capitulo se presenta una extensión de esas mediciones para el $\mathcal{L E L}$ como un todo. Esto no significa que no se puedan proponer mediciones que sean de aplicación exclusiva del LEL, sino que a partir de las mediciones establecidas para las componentes (simbolos) se definirán medidas aplicadas al conjunto (LEL)
\end{abstract}

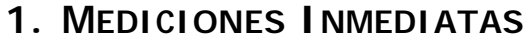

Definición 1: dado un $\mathcal{L E L} L$ se indica con $C S \mathcal{L}(\mathcal{L})$ la cantidad de simbolos que se definieron.

Aplicando esta medición al $\mathcal{L E L}$ "Hotel" se ob tie ne:

$$
\operatorname{CS} \mathcal{L}(\text { Hotel })=39
$$

La cantidad de entradas que contiene este diccionario llamado LEL es una pauta de su tamaño, pero no es suficiente en símismo para comparar dos LEL $y$ decir que el tamaño de uno es mayor que el delotro.

La cantidad de simbolos que componen el LEL (al igual que la cantidad de palabras que intervienen en la definición de un simbolo) es una "medida ingenua" e inmediata del tamaño. La cantidad de entradas necesarias para comprender un dominio depende de las habilidades técnicas del ingeniero de requerimientos para capturar el conocimiento, es decir, que en la cantidad de simbolos definidos entra en juego la subjetividad del individuo que realice el trabajo. Por otro lado, en sucesivas entrevistas con los usuarios, la cantidad de simbolos iráfluctuando en más o menos en función del intercambio de información que se realice. Y, finalmente, al considerar la utilización de simbolos en la definición de otros simbolos, se puede concluir que algunos simbolos no se usan, con lo cual puede suceder que no sean relevantes para el UdD o bien que tengan una tasa de uso muy baja y que por lo tanto se pueda considerar la posibilidad de eliminarlos. Con lo antedicho se pretende explicitar el hecho de que la cantidad de simbolos del LEL, CSL, no es una medición rigurosa en el sentido de proveer un medio inobjetable al comparar el tamaño de diferentes $\mathcal{L E L}$.

Las definiciones 2 y 3 adolecen de las mismas limitaciones ya que se basan en la misma técnica de medición. 
Definición 2: dado un $\mathcal{L E L} \mathcal{L}$ se indic a con $\mathcal{D S} \mathcal{L C}\left(\mathcal{L}, C_{k}\right)$ la cantidad de simbolos en cada grupo $\mathcal{C}_{k}$ de una clas ificación dada, donde $K=\mathcal{S}, \mathcal{V}, O, \mathcal{E}$.

En [Hadad '97] se sugiere utilizar una clasificación general para los simbolos del LEL formada por: Sujeto, Verbo, Objeto y Estado. En nuestro caso será $\mathcal{C}_{\mathcal{S}}=$ Sujeto, $C_{v}=$ Verbo, $C_{o}=$ Obje to y $C_{\mathcal{E}}=$ Estado.

Teniendo en cuenta esta clasificación, se tendrá para la categoría Sujeto del $\mathcal{L E} \mathcal{L}$ "Hote l":

$$
\mathcal{D S} \mathcal{L C}(\mathcal{H o t e l} \text {, suje to })=2
$$

Puesto que cada uno de los simbolos del LEL forman efectivamente parte de alguno de los grupos de la clasificación propuesta, [Hadad '97], [Leite '0o] entonces se de be cumplir que la cantidad de simbolos que forman un LEL L sea igual a la suma de la cantidad de los simbolos de cada categoría de la clasificación propuesta:

$$
\operatorname{CS} \mathcal{L}(\mathcal{L})=\sum_{\substack{k=S, O \\ \mathcal{V}, \mathcal{E}}} \operatorname{DS} \mathcal{L C}\left(\mathcal{L}, \mathcal{C}_{k}\right)
$$

Eje mplificando con el $\mathcal{L E L} \mathcal{H}$ otel se ob tendrá:

$\operatorname{CSL}(\mathcal{H}$ tel $)=\operatorname{DS} \mathcal{L C}(\mathcal{H o t e l}$, Verbo $)+\mathcal{D S} \mathcal{L C}(\mathcal{H o t e l}$, objeto $)+\mathcal{D S} \mathcal{L C}(\mathcal{H}$ tetel, Suje to $)+$ $\mathcal{D S} \mathcal{L C}(\mathcal{H o t e l}$, Estado $)=12+25+2+0=39$

Definición 3: dado un $\mathcal{L E L} \mathcal{L}$ se indic a con $C P \mathcal{L}(\mathcal{L})$ la cantidad de palabras del LEL $\mathcal{L} q u e$ se usan para definir todas las entradas.

$S$ i la cantidad total de entradas del LEL L es $\mathcal{C S L} \mathcal{L} L$ y $x_{i}$ es una de las entradas, entonces la cantidad de palabras se puede expresar como:

$$
\operatorname{CPL}(\mathcal{L})=\sum_{i=1}^{\operatorname{CSL}(\mathcal{L})} \operatorname{CP}\left(x_{i}\right)
$$

Para el LEL "Hotel" se obtendrá entonces:

$$
\operatorname{CPL}(\mathcal{H o t e l})=2616
$$

siendo este valor la cantidad de palabras usadas para definir todas las entradas.

$\mathcal{A}$ partir de la Definición 4 hasta la Definición 7 se introduce el concepto de palabra normalizada para la contabilidad, teniendo asi estas mediciones un carácter más estricto.

Definición 4: dado un $\mathcal{L E} \mathcal{L} \mathcal{L}$ se indica con $C P \mathcal{N}(\mathcal{L}(\mathcal{L})$ la cantidad de palabras normalizadas del $\mathcal{L E L} \mathcal{L}$ que se usan para definir todas las entradas.

Esta magnitud se puede calcular más fácilmente utilizando la siguiente fórmula: 


$$
\operatorname{CPN}\left(\mathcal{L}(\mathcal{L})=\sum_{i=1}^{\operatorname{csL}(\mathcal{L})} \operatorname{CPN}\left(x_{i}\right)\right.
$$

siendo $\chi_{i}$ una entrada de l $\mathcal{E L} \mathcal{L}$ y $C S \mathcal{L}(\mathcal{L})$ la cantidad total de entradas

Aplicando es ta fórmula al $\mathcal{L E L}$ "Hotel" se tendrá:

$$
\operatorname{CPN}(\mathcal{L}(\text { Hotel })=1035
$$

que es la cantidad de palabras usadas para definir todas las entradas del LEL "Hotel" que pasaron el proceso de normalización y no fue ron eliminadas.

Definición 5: dado un $\mathcal{L E L} \mathcal{L}$ se indic a con $C \mathcal{V} \mathcal{N}(\mathcal{L})$ la cantidad de ve rbos normalizadas del $\mathcal{L E L} \mathcal{L}$ que usa para expresar todas las entradas.

Nuevamente el cálculo resulta más sencillo si se aplica una sumatoria a las mediciones calculadas para cada simbolo, entonces se tendrá que:

$$
C \mathcal{V} \mathcal{N} L(\mathcal{L})=\sum_{i=1}^{\operatorname{cSL}(\mathcal{L})} \operatorname{CVN}\left(x_{i}\right)
$$

siendo $x_{i}$ una entrada del $\mathcal{L E L} \mathcal{L}$ y $C S \mathcal{L}(\mathcal{L})$ la cantidad total de entradas.

Para el ejemplo del LEL Hotel se ob tendrá:

$$
\operatorname{CVN}(\mathcal{L}(\text { Hotel })=230
$$

que es la cantidad de verbos usados en la definición de todas las entradas del $L \mathcal{E}$ "Hotel" que pasaron el proceso de normalización y no fueron eliminados.

Definición 6: dado un LEL $\mathcal{L}$ se indica con CSUL(L) la cantidad de simbolos usados en el $\mathcal{L E L} L$ para de finir todas las entradas.

Esta medición considera la cantidad de veces que se usaron simbolos del $L \mathcal{E L}$ para definir todos los simbolos de un LEL dado, lo que significa que habrá simbolos que se cuentan varias veces.

También en este caso se propone una sumatoria de los valores obtenidos. Cabe recordar que la cantidad de simbolos usados para definir el LEL, CS UL(L) es igual a la sumatoria del Fan In de todos los simbolos del LEL, entonces se tiene que:

$$
\operatorname{CS} \mathcal{U L}(\mathcal{L})=\sum_{i=1}^{\operatorname{CSL}(\mathcal{L})} \operatorname{CS}\left(x_{i}\right)=\sum_{i=1}^{\operatorname{CSL}(\mathcal{L})} \mathcal{F} I\left(x_{i}\right)
$$

siendo $x_{i}$ una entrada del $\mathcal{L E L} \mathcal{L}$ y $C S \mathcal{L}(\mathcal{L})$ la cantidad total de entradas del $\mathcal{L E L} \mathcal{L}$

Aplicando esta fórmula al $\mathcal{L E L}$ "Hotel" se obtendrá la cantidad de simbolos que se usaron para definir el total de los simbolos

$$
\text { CS UL(Hotel })=332
$$


Definición 7: dado un LEL $\mathcal{L}$ se indica con COPN $\mathcal{L}(\mathcal{L})$ la cantidad de otras palabras normalizadas del $\mathcal{L E L} \mathcal{L}$ que se usan para definir todas las entradas.

Aplicando el mismo criterio que se usó al definir esta magnitud para el simbolo del $\mathcal{L E} \mathcal{L}$, se establece la fórmula:

$$
\operatorname{CPN} \mathcal{L}(\mathcal{L})=\operatorname{COPN} \mathcal{L}(\mathcal{L})+\operatorname{CVN} \mathcal{L}(\mathcal{L})+\operatorname{CS} \mathcal{U L}(\mathcal{L})
$$

Volviendo al ejemplo del LEL "Hotel" se tie ne que:

$$
\operatorname{COP\mathcal {N}}(\mathcal{H o t e l})=1035-230-332=473
$$

es la cantidad de otras palabras normalizadas que se usaron para definir el total de los simbolos.

\section{MEDICIONES CALCULADAS}

Relacionando las mediciones mediante la aplicación de fórmulas se pueden efectuar cálculos y se espera poder inferir caracteristicas del sistema.

La estadistica ofrece una amplia gama de mediciones indirectas que pueden resultar muy útiles si se aplican correctamente. Esto significa que es importante conocer las limitaciones que cada fórmula tenga, las restricciones que tiene para su aplicación y fundamentalmente realizar una buena interpretación de los resultados, pues se puede llegar a conclusiones irreales sino se es cuidadoso en este punto.

Muchas de las pruebas estadisticas que se pueden aplicar, se calculan automáticamente usando planillas electrónicas, por lo tanto no es necesario conocer a fondo la teoría para implementarlas, lo único fundamentalmente importante es saber si las prue 6 as son apropiadas para los datos en cuestión.

$\mathcal{E l}$ propósito del análisis de datos es evidenciar cualquier relación o patrón entre ellos, de tal forma que sirvan para evaluar los atributos que se están midiendo [Fenton'96].

Las mediciones estadísticas ${ }^{1}$ se aplican a cada grupo de la clasificación ge ne ral (es decir como una subpoblación) y también para elconjunto de todas las entradas del $\mathcal{L E L}$ (es decir para la población).

Para este trabajo se seleccionaron algunas mediciones estadisticas que se consideraron de posible utilidad (esto se verificará al realizar el correspondiente anális is de los resultados en función de los datos) para cuantificar el tamaño del $L \mathcal{L} L$ o para inferir características y comportamientos relacionados con el tamaño. Se calcularon para la CPN CVN CS y COPN la media aritmética, la me diana, la moda, el desvio estándar y elcoeficiente de determinación como indicadores de la distribución de las palabras normalizadas en el LEL. Utilizando los resultados de la media, la mediana y la moda se de termina la forma de la distribución. Se realizaron diagramas de dispersión, se trazó la líne a de regresión entre conjuntos de datos y finalmente se

${ }^{1}$ El Apéndice D contie ne el detalle de las fórmulas estadísticas utilizadas. 
calcularon los coeficientes de determinación para establecer la existencia de una relación entre los conjuntos de datos seleccionados.

Se tomaron los datos que computan todas las ocurrencias de las palabras normalizadas, según se establece en el Capitulo VI. Se ha observado que los resultados difieren levemente de los que se obtendrían usando los datos que no contabilizan las repeticiones, esto se debe a que se trabaja sólo con las palabras normalizadas entonces las definiciones son precisas y concretas. Por todo lo antedicho es que se prefirió utilizar la totalidad de la información ya que resulta más representativa de la realidad.

Dado que la cantidad de datos es pequeña, se trabajará con la población y no con una muestra.

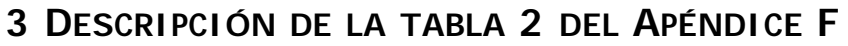

Para la obtención de los datos estadísticos, se usó la planilla de cálculo Excel 2000 de Microsoft versión 9.0.2812

La Figura VII.1 muestra parte de la Tabla 2 del Apéndice $\mathcal{F}$ que contiene las mediciones aplicadas al LEL "Hotel". En la parte superior se consignan las mediciones estadísticas propuestas, aplicadas a cada grupo según la clasificación general.

\begin{tabular}{|c|c|c|c|c|c|c|c|}
\hline & $C P$ & CPN & CVN & CS & COPN & $D S L C$ & ENTRADAS \\
\hline \multirow{19}{*}{ 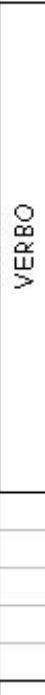 } & 61 & 17 & 4 & 10 & 3 & & CANCELACION DE RESERVA \\
\hline & 194 & 75 & 15 & 27 & 33 & & $C \mathrm{HECK}$ IN \\
\hline & 133 & 48 & 9 & 17 & 22 & & CHECK OUT \\
\hline & 87 & 30 & 5 & 14 & 11 & & FACTURACION \\
\hline & 68 & 23 & 5 & 10 & 8 & & No SHOW \\
\hline & 85 & 33 & 10 & 9 & 14 & & PAGO \\
\hline & 52 & 21 & 6 & 7 & 8 & & PEDIDO DE ALOJAMIENTO \\
\hline & 49 & 21 & 6 & 5 & 10 & & PEDIDODE LIMPIEZA EXTRA \\
\hline & 56 & 21 & 6 & 5 & 10 & & P. MANTENIMIENTO EXTRA \\
\hline & 55 & 23 & 5 & 6 & 12 & & P. SERVICIO DE DESPERTADOR \\
\hline & 53 & 24 & 6 & 5 & 13 & & REPOSICION DE INSUMOS \\
\hline & 117 & $49^{\circ}$ & 8 & 17 & 24 & & SOLICITUD DE RESERVA \\
\hline & & & & & & 12 & \\
\hline & & 32 & 7 & 11 & 14 & & MEDIA \\
\hline & & 24 & 6 & 10 & 12 & & MEDIANA \\
\hline & & 21 & 6 & 5 & 8 & & MODA \\
\hline & & 16 & 3 & 6 & 8 & & DESVST \\
\hline & & 0.51 & 0.42 & 0.58 & 0.57 & & COEFICIENTE DE VARIACION \\
\hline & 31 & 17 & 3 & 7 & 12 & & ADMTNTSTRACTON \\
\hline
\end{tabular}

Figura VII.1: Tabla 2 del Apéndice $\mathcal{F}$ Mediciones estadísticas

En el final de la tabla se consignan los resultados obtenidos al aplicar las me diciones a la población, es decir a todo el LEL, tal como lo indic a la Figura VII.2 


\begin{tabular}{|c|c|c|c|c|c|c|}
\hline \multirow{6}{*}{$\begin{array}{l}z \\
0 \\
0 \\
0 \\
0 \\
0 \\
0 \\
0\end{array}$} & 1035 & 230 & 335 & 470 & 39 & TOTAL \\
\hline & 27 & 6 & 9 & 12 & & MEDIA \\
\hline & 23 & 6 & 7 & 11 & & MEDIANA \\
\hline & 30 & 6 & 5 & 8 & & MODA \\
\hline & 13 & 3 & 6 & 7 & & DESVST \\
\hline & 0.49 & 0.49 & 0.65 & 0.55 & & COEFICIENTE DE VARIACION \\
\hline
\end{tabular}

Figura VII.2: Tabla 2 del Apéndice F: Mediciones para la Población

La Figura VII.3 muestra las funciones de la planilla de cálculo Excel 2000 utilizadas para calcular las determinaciones propuestas.

\begin{tabular}{|c|l|c|c|c|c|l|}
\hline & & & 12 & \\
\hline & -PROMEDIO(D67:D77) & 5 & 7 & 11 & & MEDIA \\
\hline & -MEDIANA(D67:D77) & 6 & 6 & 11 & & MEDIANA \\
\hline & -MODA(D67:D77) & 6 & 5 & MOA & & MODA \\
& -DESVESTR(D67:D77) & 2 & 5 & 7 & & DESVST \\
\hline & -D83/D79 & 0.46 & 0.67 & 0.61 & & COEFICIENTE DE VARIACION \\
\hline 31 & 17 & 3 & 2 & 12 & & ADMINISTRACION \\
\hline
\end{tabular}

Figura VII.3: Tabla 2 del Apéndice F: Funciones de la planilla de cálculo 


\section{CAPITULO VIII}

\section{CASO DE ESTUDIO}

En este capítulo se analizarán los resultados de la aplicación a un caso de estudio de las mediciones desarrolladas en los Capítulos VI "Mediciones aplicadas a las palabras normalizadas del símbolo" y VII "Mediciones aplicadas a las palabras normalizadas del LEL".

El caso de estudio elegido es el LEL "Hotel" cuyo proceso de elaboración se explicó en el Capítulo II "Elicitación de Requerimientos" y la versión completa se puede consultar en el Apéndice $E$.

\section{MEDICIONES APLICADAS AL SÍMBOLO}

Las definiciones a las que se hace mención en este apartado se pueden consultar en el Capítulo VI.

\subsection{MEDICIONES INMEDIATAS ${ }^{1}$}

Son las que surgen de la simple y directa observación de un texto escrito en Lenguaje Natural.

\subsubsection{CANTIDAD PALABRAS}

En primer lugar, si el objetivo es medir tamaño, instintivamente el analista contará las palabras que contiene el texto sometido a medición, habrá obtenido así la cantidad de palabras, simbolizado por $C P$, según se indica en la Definición 1. Esta medición está fuertemente influenciada por el estilo de redacción utilizado por el ingeniero de requerimientos que realizó la elicitación. Por lo tanto, a la hora de comparar el tamaño de diferentes LEL, esta medición carecerá de valor práctico. Es así que surge la necesidad de establecer otros mecanismos de medición para lo cual, tal como se explicó exhaustivamente en el Capítulo V, se definió el concepto de Palabra Normalizada.

\subsubsection{CANTIDAD DE ORACIONES}

La aplicación de la Definición 2 permite obtener otra medida inmediata que es la cantidad de oraciones que se usaron para definir un concepto indicado con $C O$.

Esta es una medición sencilla de realizar y cobra mayor valor cuando se la asocia con la cantidad de verbos normalizados. Si se piensa que cada oración expresa un idea o concepto dentro de la definición, entonces se puede inferir que una definición con un $\mathrm{CO}$ alto es importante.

\footnotetext{
${ }^{1}$ La Tabla 1 del Apéndice F contiene las mediciones efectuadas sobre cada símbolo del LEL
} 
Entradas como PASAJERO/HUÉSPED/PAX, RECEPCIONISTA y CHECK IN tienen la cantidad de oraciones más alta de este LEL, 14, 13 y 13 respectivamente.

\subsubsection{MEDICIONES APLICADAS a LAS PALABRAS NORMALIZADAS}

La Definición 3 de Palabra Normalizada, permite realizar una primera aproximación acerca del tamaño de un símbolo contando sólo las palabras que luego de ser sometidas al proceso de normalización que indica la Definición 4, no fueron eliminadas, obteniéndose así la cantidad de palabras normalizadas, tal como lo explica la Definición 5, que será la medición fundamental en el contexto de esta tesis.

En una segunda etapa estas palabras normalizadas se clasifican teniendo en cuenta sus características lingüísticas en: verbos normalizados, originando la medición CVN, símbolos incluidos en la definición, simbolizado por CS y otras palabras normalizadas que determina el valor de COPN. La combinación de algunas de estas mediciones dará un conjunto de coeficientes.

Los resultados que se obtengan permitirán caracterizar el LEL en estudio para luego poder elaborar conclusiones generales.

\subsubsection{CANTIDAD DE PALABRAS NORMALIZADAS}

Como ya se ha dicho, la cantidad de palabras $C P$ no es una medición rigurosa pues depende fuertemente de la forma de redactar del ingeniero de requerimientos que haya hecho el análisis del UdD. Sin embargo, se utiliza como parámetro de referencia para evaluar características del LEL que permitan hacer inferencias sobre su tamaño. Esto quedará evidenciado al calcular el coeficiente de redundancia, COER que se hace en el parágrafo 1.2.1.

La Definición 5 explica el concepto cantidad de palabras normalizadas indicado con CPN.

En la Tabla VIII.1 se reproducen los valores extremos máximo y mínimo encontrados para la cantidad de palabras CP y la cantidad de palabras normalizadas CPN. Nótese que los valores no corresponden necesariamente a la misma entrada.

Por ahora interesa resaltar el hecho de que el valor máximo registrado corresponde a $57 \%$, esto indica que del total de las palabras usadas este porcentaje corresponde a palabras normalizadas y se da para la entrada LISTA DE PRECIOS, y para MANTENIMIENTO con un valor similar $(56 \%)$, mientras que el caso del mínimo corresponde a la entrada CANCELACIÓN DE RESERVA, e indica que en esta entrada sólo el $28 \%$ de las palabras son normalizadas.

\begin{tabular}{|c|c|c|c|}
\cline { 2 - 4 } \multicolumn{1}{c|}{} & CP & \multicolumn{2}{c|}{ CPN } \\
$\%$
\end{tabular}

Tabla VIII.1: Mediciones extremas registradas para CPN (no corresponden a la misma entrada) 


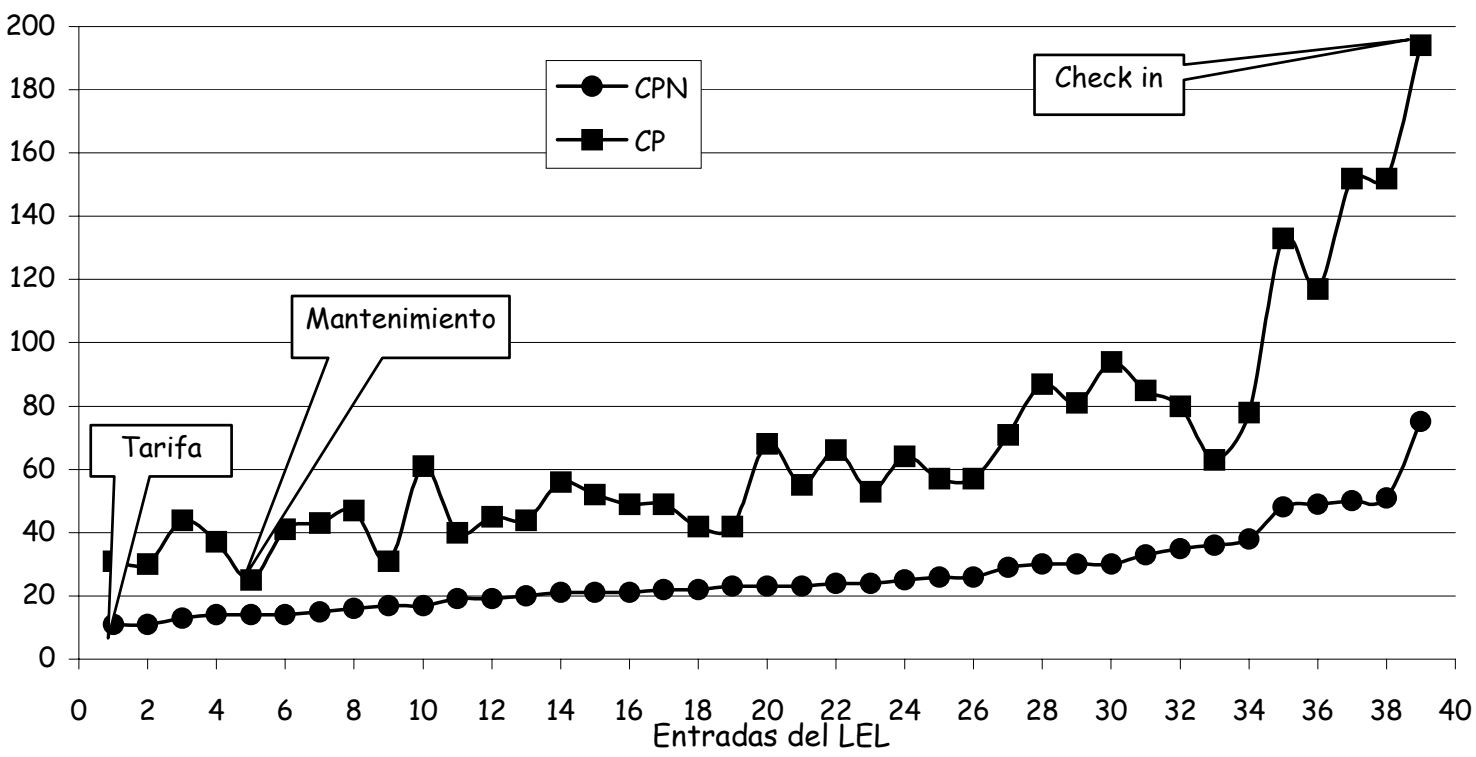

Figura VIII.1: Distribución de la CP y CPN de las entradas del LEL, ordenados por CPN creciente

Se observa en la Figura VIII.1 que para la cantidad de palabras normalizadas, los valores extremos corresponden a $C P N(C H E C K$ IN $)=75$ y CPN $($ TARIFA $)=$ CPN(FICHERO DE PASAJEROS) $=11$ y para la cantidad de palabras, CP(CHECK IN) $=194$ pero el mínimo corresponde a $C P(M A N T E N I M I E N T O)=25$, es decir solo coinciden los valores extremos en el caso de la entrada CHECK IN.

Se destaca la entrada MANTENIMIENTO por presentar la menor diferencia entre los valores de cantidad de palabras y cantidad de palabras normalizadas, CPN $($ MANTENIMIENTO $)=14$ y CP(MANTENIMIENTO $)=25$.

El $87 \%$ de las entradas tienen entre 10 y 40 palabras normalizadas en su definición.

Es llamativo el hecho de que la línea que une todas las entradas para la cantidad de palabras normalizadas sigue en forma bastante similar la forma de la línea que une la cantidad de palabras de todas las entradas. Aún teniendo en cuenta que las entradas están ordenadas en forma creciente según CPN, el crecimiento es gradual y sin marcadas diferencias entre entradas sucesivas, esto podría sugerir que la cantidad de palabras normalizadas es, efectivamente una medida que permite comparar el tamaño de diferentes entradas y está relacionada de alguna manera con la cantidad de palabras para cada entrada.

Ahora bien, las clases de palabras (verbos, símbolos y otras palabras) que intervienen en una definición del LEL tienen particularidades que no necesariamente siguen el comportamiento del conjunto de palabras normalizadas.

Se han representado en la Figura VIII.2 las entradas del LEL. Cada barra corresponde a una de ellas y tiene como longitud la cantidad de palabras normalizadas, además se distinguen las cantidades de verbos, símbolos y otras palabras normalizadas con diferentes texturas. 


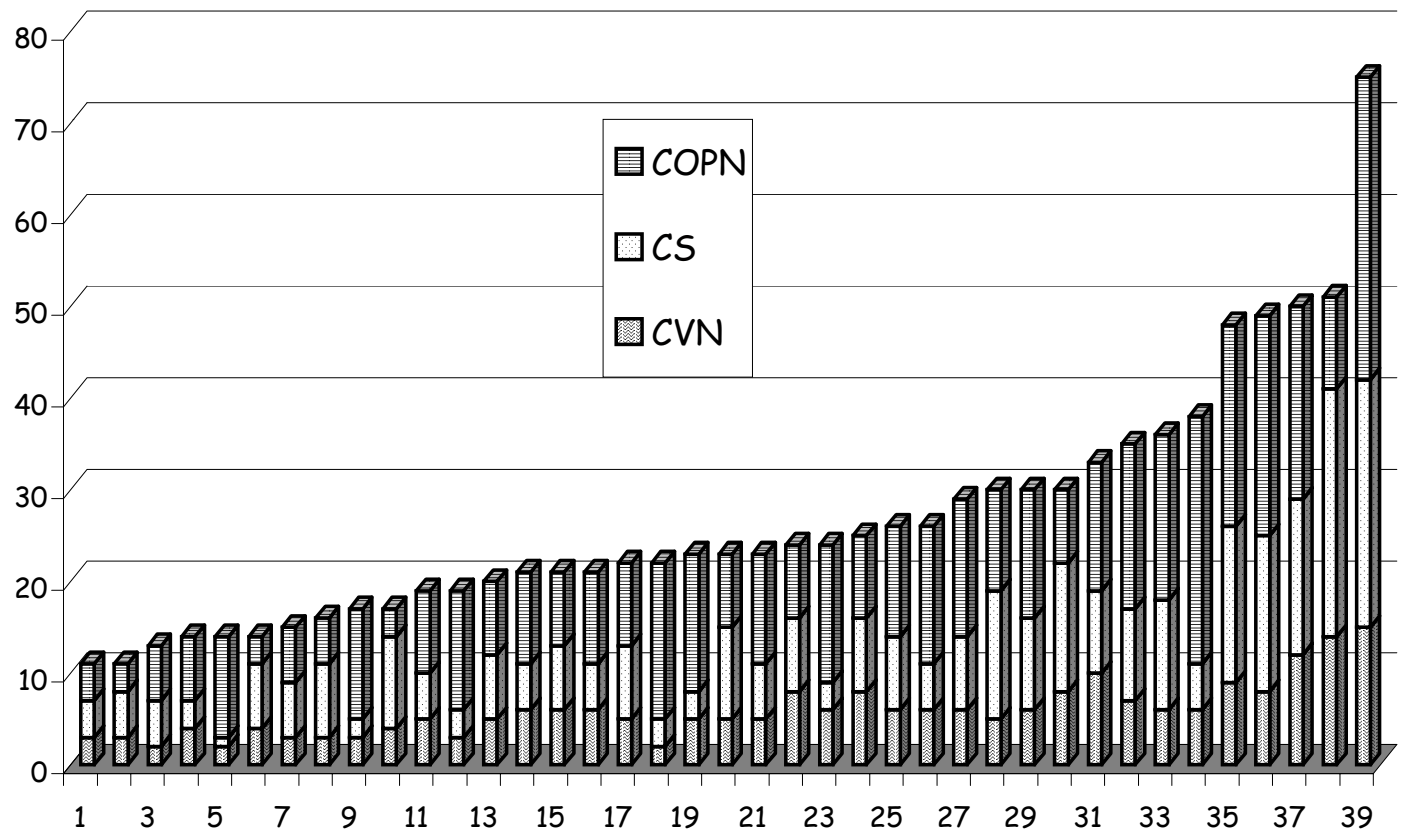

Figura VIII.2: Distribución de la CPN de las entradas del LEL, ordenados por CPN creciente

Al comparar el tamaño correspondiente a cada clase de palabra en la Figura VIII.2 se destacan las entradas de los números 38 y 39, que corresponden al símbolo RECEPCIONISTA y CHECK IN respectivamente, en las que la cantidad de símbolos (CS) y la cantidad de verbos (CVN) tienen el mismo tamaño aún cuando la longitud total de la columna, es decir la cantidad de palabras normalizadas, correspondiente a la entrada 39 es mucho mayor. Entonces se diferencian sólo en la cantidad de otras palabras normalizadas (COPN). Indudablemente en el dominio en cuestión son entradas que juegan un papel determinante. Estos valores podrían sugerir la identificación de entradas clave en una asociación entre tamaño e importancia.

Otra entrada particular, a partir de la Figura VIII.2, resulta ser la 5 que representa al símbolo MANTENIMIENTO, la que parece no poseer componentes de cantidad de símbolos (CS), aunque en realidad la Tabla 1 del Apéndice F registra que tiene 1. Esto podría ser un indicador de un símbolo con deficiencias en su redacción o poca conexión con el resto de las entradas del LEL.

Por todo lo analizado hasta ahora, se podría inferir que la entrada CHECK IN tiene características especiales que la diferencian del conjunto de símbolos y por lo tanto, en virtud de tender a obtener resultados generales, se podría obviar.

En la Figura VIII.3 se graficó una barra por cada entrada y en cada una se representó la distribución porcentual de cada clase de palabra normalizada. Al comparar cada barra se concluye que la mayor variabilidad en cuanto a la composición en porcentaje de la cantidad de palabras normalizadas se da para la cantidad de otras palabras normalizadas (COPN) que presenta fluctuaciones importantes, especialmente notable en los casos de las entradas 5, 9, 12,18 y 34 en las que la COPN representan entre el 70 y el $80 \%$ del total de las palabras normalizadas (CPN). 


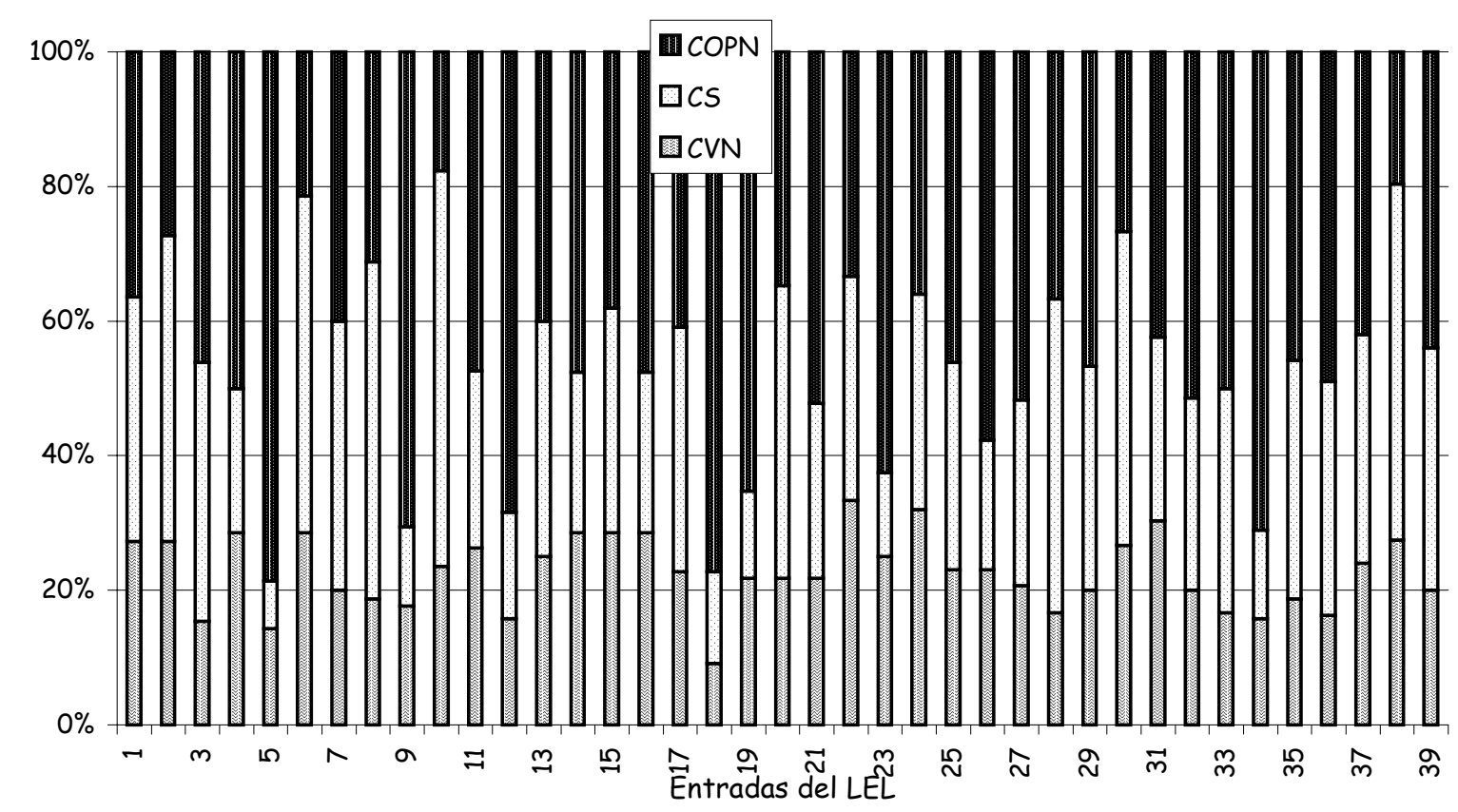

Figura VIII.3: Composición porcentual de la CPN, ordenados por CPN creciente

Por otro lado, la cantidad de verbos normalizados, CVN, se mantiene acotado entre valores muy similares entre sí. La cantidad de símbolos, CS, tiene un comportamiento intermedio entre CVN y COPN, sin presentar valores tan extremos como lo hace la COPN. En el análisis de composición porcentual, la entrada 39 correspondiente a CHECK IN, no presenta ninguna singularidad.

Hasta ahora parecería un hecho lógico afirmar que la mayor estabilidad de los valores medidos corresponda a la cantidad de verbos, pues su dependencia del estilo de la redacción es menor que la que tienen los símbolos utilizados y por supuesto la cantidad de otras palabras normalizadas.

A continuación se hará un análisis específico para cada uno de los tipos de palabras normalizadas.

\subsubsection{CANTIDAD DE VERBOS NORMALIZADOS}

\begin{tabular}{|l|c|c|}
\multicolumn{2}{l|}{} & $\%$ \\
\hline MÁXIMO & 15 & 33 \\
\hline MINIMO & 2 & 9 \\
\hline
\end{tabular}

Tabla VIII.2: Mediciones extremas registradas para CVN (no corresponden a la misma entrada)

Las características de esta medición se indican en la Definición 7.

Los valores extremos para la cantidad de verbos normalizados son, según la Tabla VIII.2, CVN(CHECK IN) $=15$ y CVN(GASTRONOMIA), CVN(MANTENIMIENTO) y CVN(RECEPCION) que alcanzan el valor 2. Estos valores dan una relación entre verbos normalizados de 1:7,5

Los porcentajes se calcularon en función de CPN, la amplitud registrada no resulta llamativa. 


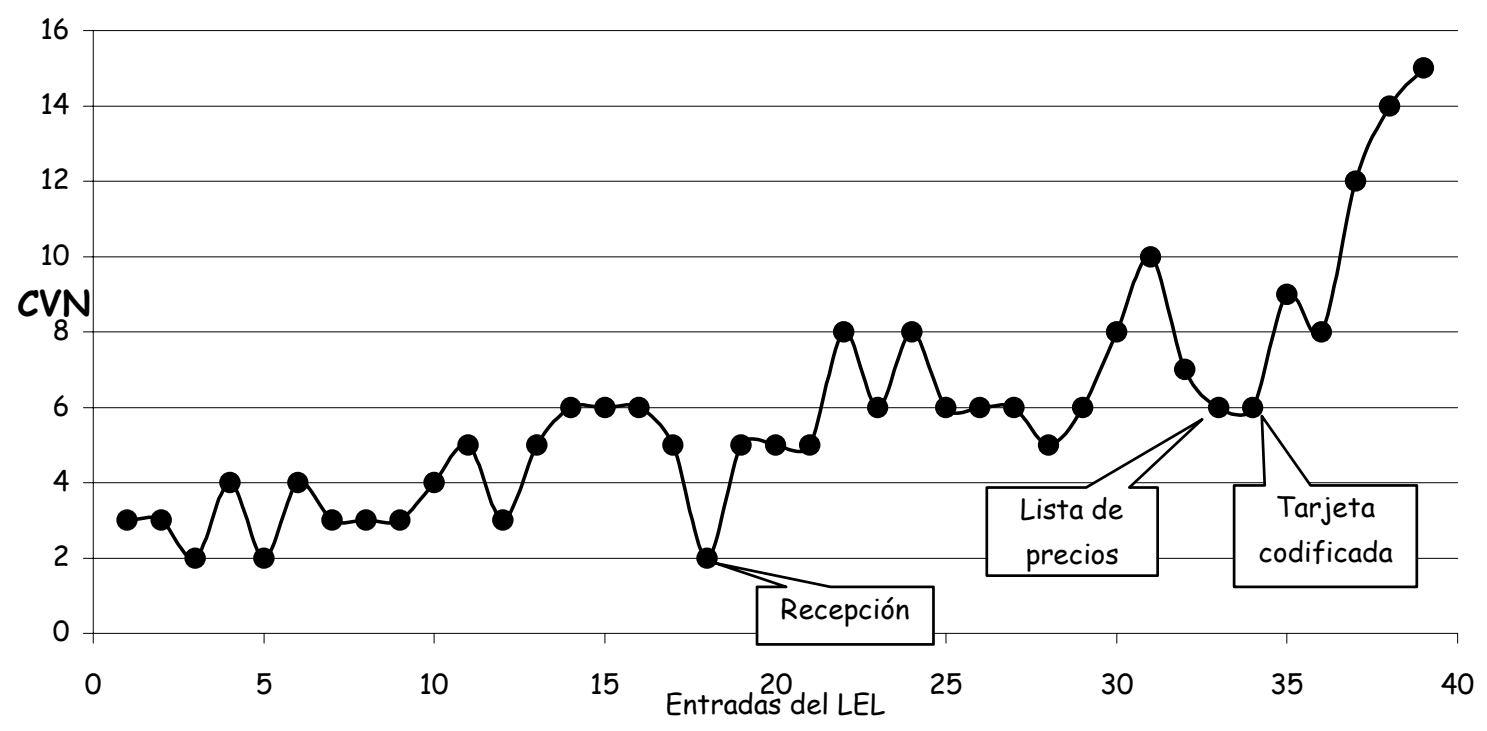

Figura VIII.4: CVN de las entradas del LEL, ordenados por CPN creciente

En la Figura VIII.4 se destacan tres valores que no siguen el crecimiento esperado, ellos son los correspondientes a las entradas RECEPCIÓN, LISTA DE PRECIOS Y TARJETA CODIFICADA. Haciendo abstracción de estas observaciones, en general, los valores parecen seguir un patrón de crecimiento que acompaña, aproximadamente, a la cantidad de palabras normalizadas.

El $80 \%$ de las entradas tiene entre 3 y 8 verbos normalizados en su definición.

\subsubsection{CANTIDAD DE SÍMBOLOS O FAN IN}

Se ha descripto en la Definición 6 y en la Definición 11 el Fan In o Cantidad de Símbolos (CS) de una entrada como una medida de la cantidad de símbolos que se utilizan en la definición de un símbolo. Las entradas con CS elevado previsiblemente deben ser las que corresponden a símbolos del LEL más complejos, que describen aspectos importantes del sistema en estudio y que hacen un buen uso del principio de circularidad. Son ejemplos de esta situación los símbolos que tienen valores extremos máximos, CS(CHECK IN) y CS(RECEPCIONISTA) que llegan a 27 y CS(PASAJERO/HUÉSPED/PAX) y CS( CHECK OUT) que alcanzan un valor de 17. Indudablemente estos símbolos describen entidades importantes del LEL "Hotel".

\begin{tabular}{|l|c|c|}
\multicolumn{2}{l|}{} & $\%$ \\
\hline MÁXIMO & 27 & 59 \\
\hline MÍNIMO & 1 & 7 \\
\hline
\end{tabular}

Tabla VIII.3: Mediciones extremas registradas para CS (no corresponden a la misma entrada)

El mínimo que consigna la Tabla VIII.3 para la cantidad de símbolos corresponde a CS(MANTENIMIENTO) $=1$ y le sigue CS (ADMINISTRACIÓN) $=2$. Símbolos con una definición simple son los que tienen un CS bajo. La relación entre la cantidad 
de símbolos es de 1:27. Las amplitudes registradas son muy grandes, tanto en los porcentajes como en las cantidades, esto podría evidenciar símbolos con marcadas diferencias en cuanto a su valor dentro del LEL del que forman parte. Los porcentajes consignados en la Tabla VIII.3 se obtuvieron en relación a CPN.

En la Figura VIII.5 se graficaron los valores correspondientes a la cantidad de símbolos usados en la definición de cada símbolo, al igual que en el caso de la cantidad de verbos, la evolución de la cantidad de símbolos muestra una tendencia creciente, aunque no acompañe estrictamente al crecimiento de la cantidad de palabras normalizadas. Una excepción a destacar es la entrada 34 correspondiente a TARJETA CODIFICADA, que en relación a la cantidad de palabras normalizadas, la cantidad de símbolos que utiliza en su definición es baja.

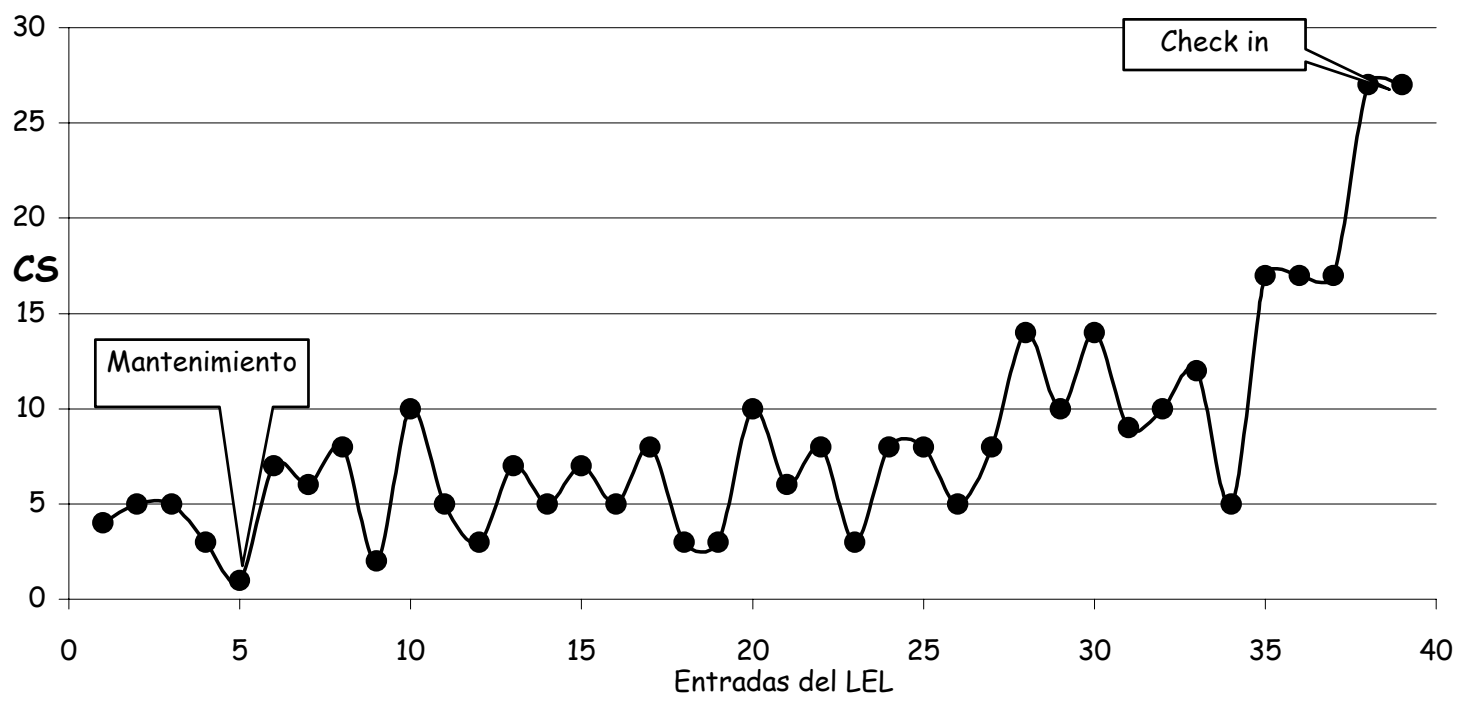

Figura VIII.5: CS de las entradas del LEL, ordenados por CPN creciente

El $82 \%$ de las entradas del LEL utilizan entre 3 y 15 entradas en su propia definición.

\subsubsection{Cantidad de OtRas Palabras Normalizadas}

\begin{tabular}{|l|c|c|}
\multicolumn{2}{l|}{} & $\%$ \\
\hline MÁXIMO & 33 & 79 \\
\hline MINIMO & 3 & 18 \\
\hline
\end{tabular}

Tabla VIII.4: Mediciones extremas registradas para COPN (no corresponden a la misma entrada)

La Definición 8 establece las características de esta medición.

Los valores obtenidos a partir de la Tabla VIII.4 del máximo registrado para otras palabras normalizadas es $\operatorname{COPN}(C H E C K$ IN) $=33$ y el mínimo es COPN(CANCELACIÓN DE RESERVA), COPN(PLANILLA DE OCUPACIÓN DE HABITACIONES) Y COPN(TARIFA) con un valor 3 . Esto da una relación entre las mediciones extremas de otras palabras normalizadas de 1:11. 
El $80 \%$ de las entradas poseen entre 5 y 20 otras palabras normalizadas en su definición.

En la Figura VIII.6 se observan oscilaciones en los valores registrados para la cantidad de otras palabras normalizadas lo cual indica que no siguen el crecimiento esperado en función de la cantidad de palabras normalizadas usadas en cada entrada (recordar que para realizar el gráfico las entradas fueron ordenadas en función de los valores de CPN crecientes), destacándose especialmente la entrada RECEPCIONISTA con una cantidad de otras palabras normalizadas mucho menor de la esperada.

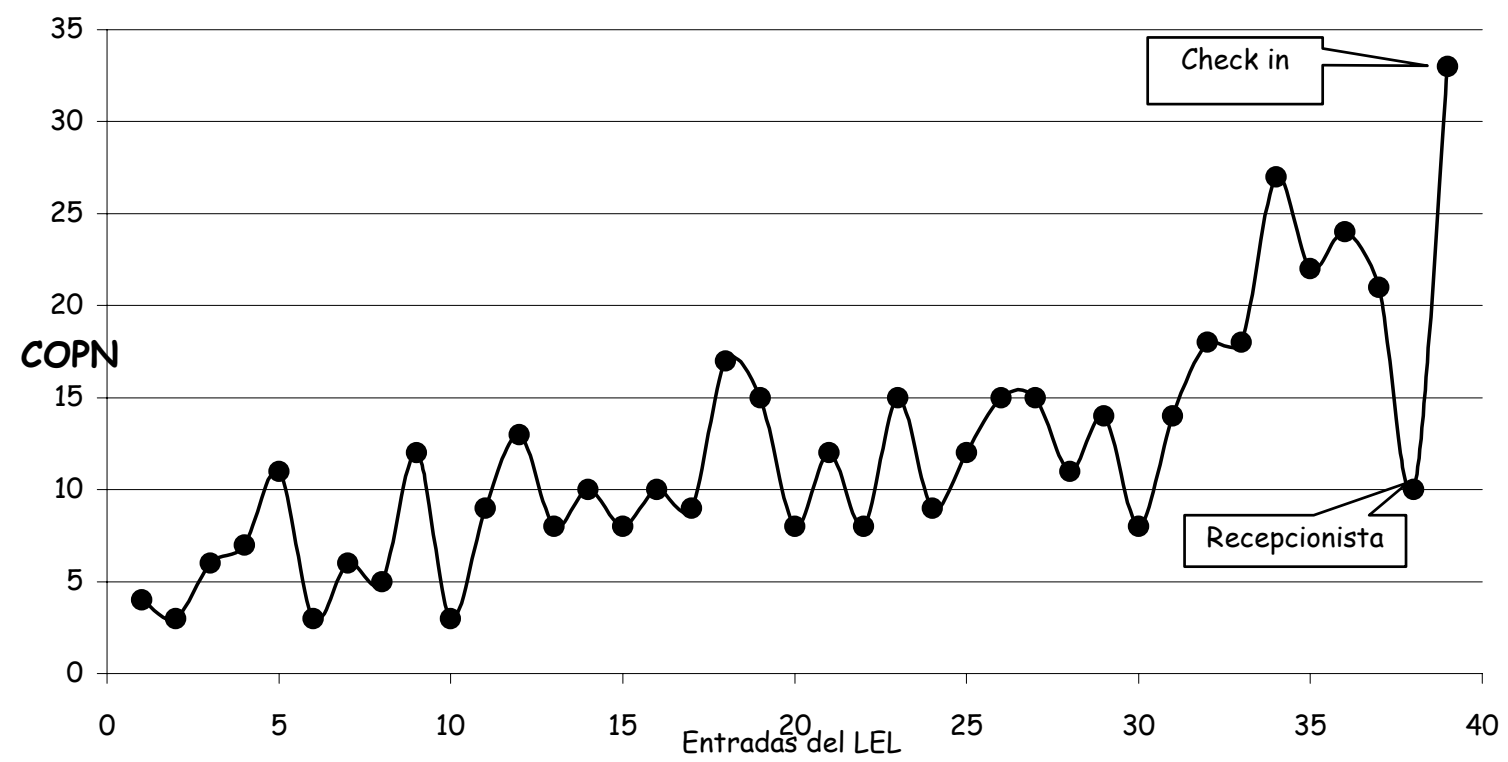

Figura VIII.6: COPN de las entradas del LEL, ordenados por CPN creciente

Como una primera aproximación se podría decir que no existe relación entre la cantidad de palabras normalizadas y la cantidad de otras palabras normalizadas. Esta afirmación será corroborada o no por cálculos posteriores.

\subsection{MEDICIONES CALCULADAS}

Estas mediciones incluyen coeficientes cuyos valores se calculan utilizando las cantidades de palabras normalizadas definidas en los parágrafos anteriores. Pretenden caracterizar los símbolos teniendo en cuenta posibles relaciones entre las clases de palabras normalizadas.

\subsubsection{COEFICIENTE DE REDUNDANCIA}

En la Figura VIII.7 se graficaron los datos calculados para el Coeficiente de Redundancia (COER) que se obtiene, para cada entrada, como el cociente $C P N(x)$ / $C P(x)$. El valor extremo mínimo corresponde a COER (CANCELACIÓN DE RESERVA) $=0,28$ y el extremo máximo a COER (LISTA DE PRECIOS) $=0,57$.

Del análisis de los valores obtenidos se puede concluir que el $82 \%$ de las entradas del LEL "Hotel" están en el rango que va desde $\operatorname{COER}(x)=0,5$ hasta 
$\operatorname{COER}(x)=0,3$. Esto significa que la cantidad de palabras normalizadas que contiene una entrada es un valor que oscila entre el 30 y $50 \%$ de la cantidad total de palabras que intervienen en su definición.

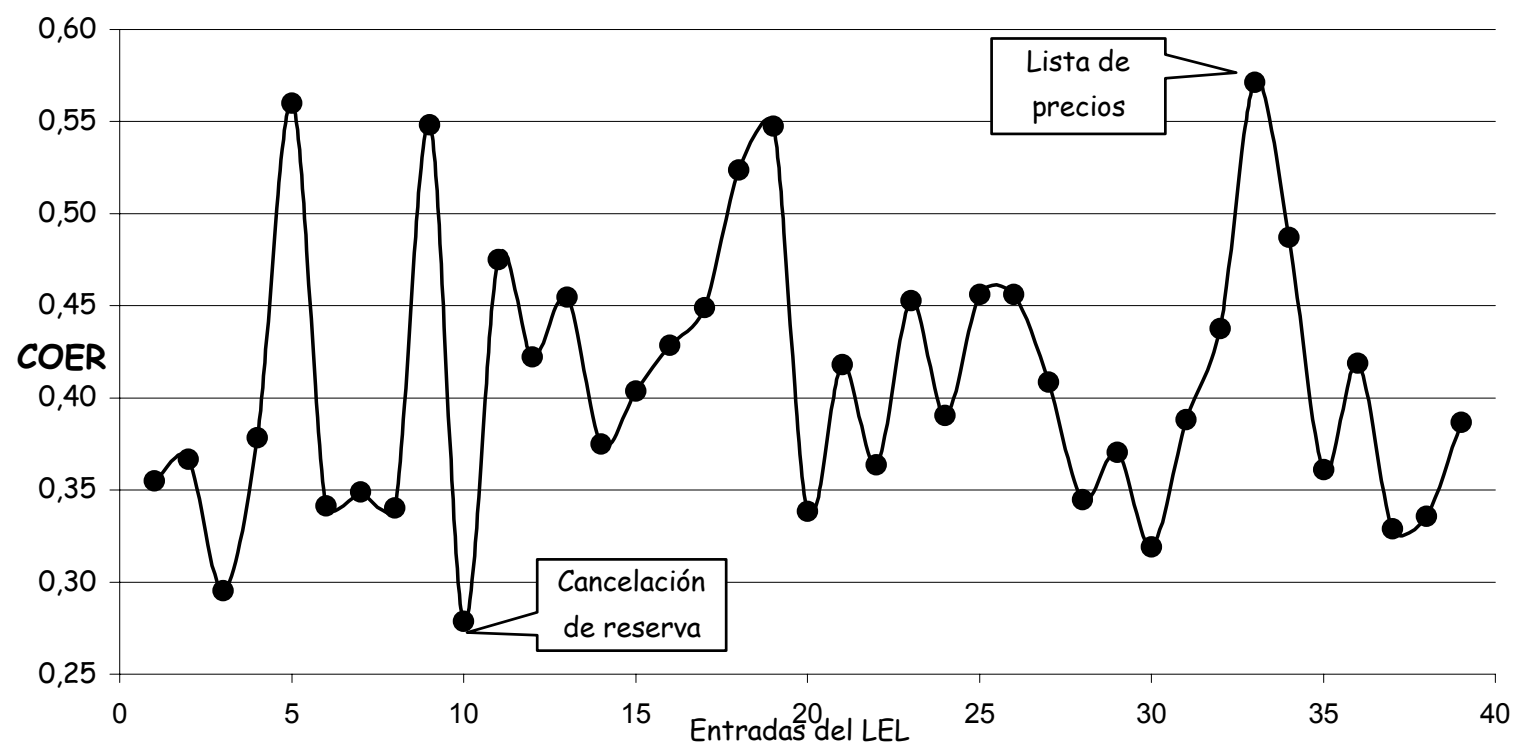

Figura VIII.7: COER de las entradas del LEL, ordenados por CPN creciente

La Definición 9 explica el coeficiente de redundancia. Este coeficiente es una magnitud que representa la estabilidad del conjunto respecto a CPN. Un COER bajo indica una entrada en la que se usaron pocas palabras normalizadas en relación a la cantidad total de palabras. Por otro lado un COER alto corresponde a una entrada en la que se hizo un buen uso del lenguaje prefiriendo palabras con fuerte valor semántico a aquéllas vacías, sin una connotación precisa. Con este coeficiente se estaría calificando el estilo de redacción y por lo tanto su calidad.

\subsubsection{COEFICIENTE DE SIMPLICIDAD}

En la Figura VIII.8 se presentan los valores calculados para el Coeficiente de Simplicidad (COES) que se obtiene, para cada entrada, como el cociente entre la Cantidad de Verbos Normalizados y la Cantidad de Oraciones usados en la definición de una entrada $(C V N(x) / C O(x))$, tal como lo explicita la Definición 10.

El $82 \%$ de las entradas están en el rango que va desde 1,2 a 0,6; esto podría denotar la utilización de una redacción simple al definir un concepto. El 37\% de las entradas contiene un verbo por oración, son los casos de $\operatorname{COES}(x)=1$.

Nuevamente, como ya se puntualizó para el coeficiente de redundancia, este coeficiente evidenciaría un estilo de expresión y por lo tanto se podría considerar una medición de calidad.

Los valores extremos corresponden a $\operatorname{COES}(P A G O)=1,67$ para el máximo y para el mínimo a COES(RECEPCIÓN) $=0,5$ 


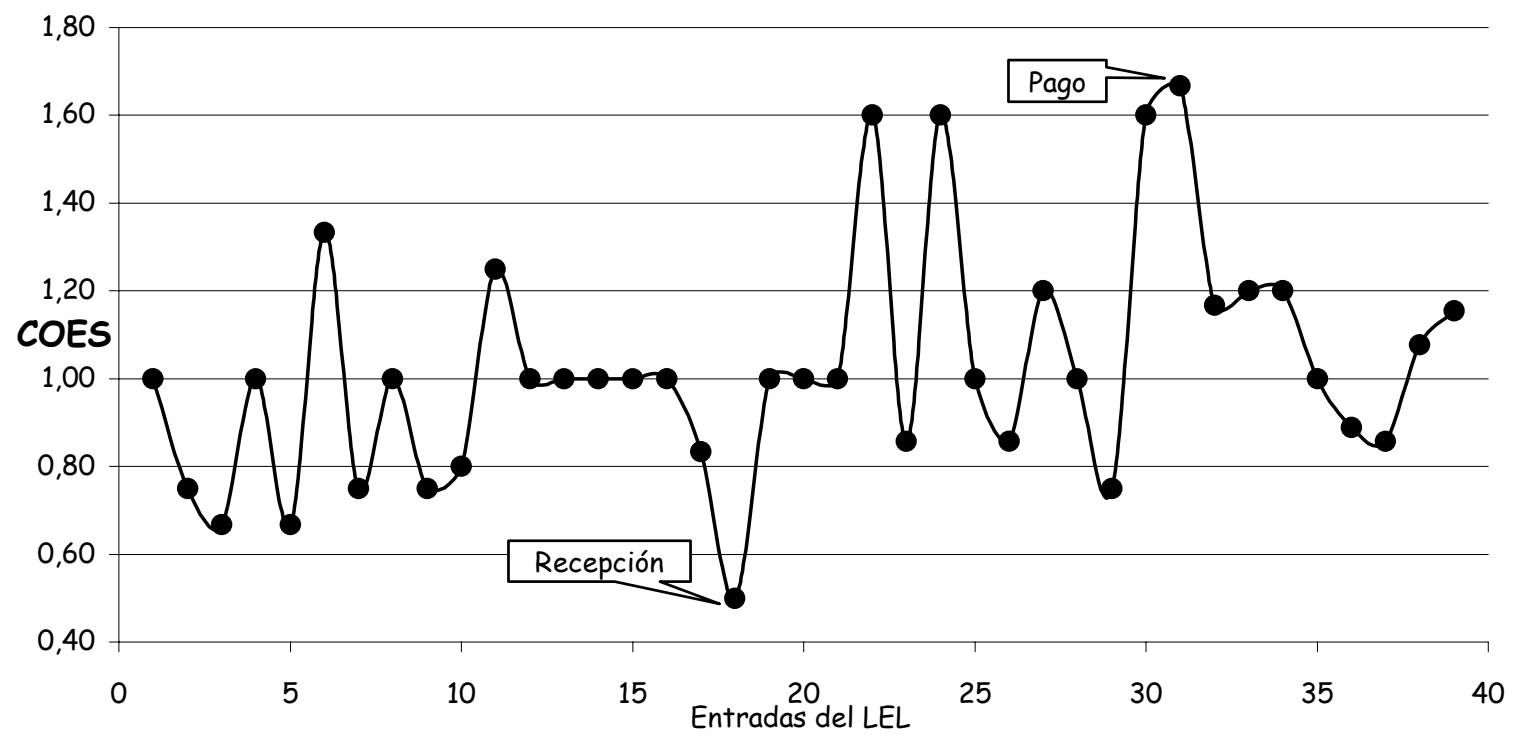

Figura VIII.8: COES de las entradas del LEL, ordenados por CPN creciente

\subsubsection{FAN OUT}

Como se indica en la Definición 12 el Fan Out (FO) representa la cantidad de veces que una entrada del LEL es usada para definir otras entradas En la Figura VIII.9 se graficaron los valores obtenidos para el caso de estudio. Puede observarse que el $82 \%$ de los valores están entre $F O(x)=10$ y $F O(x)=2$.

Si se asocia la utilización de una entrada como una medida de su importancia, es notable la presencia de entradas que serían de mayor importancia que otras. En este caso, FO(PASAJERO/HUÉSPED/PAX) $=72$ que es uno de los actores principales es el valor más alto, le siguen con valores mucho menores FO(RECEPCIÓN) $=31 \mathrm{y}$ $F O($ HABITACIÓN $)=30$, pero que indudablemente son dos conceptos clave en el dominio en cuestión. Del total de entradas, el $85 \%$ poseen un FO menor que 10.

FO(MANTENIMIENTO) $=1$ es el valor más bajo y corresponde a una entrada que se ha usado una sola vez en la definición de otras entradas. Puede afirmarse que un símbolo con un FO bajo no es importante en la modelización de este UdD y que habría que considerar la posibilidad eliminarlo del LEL. 


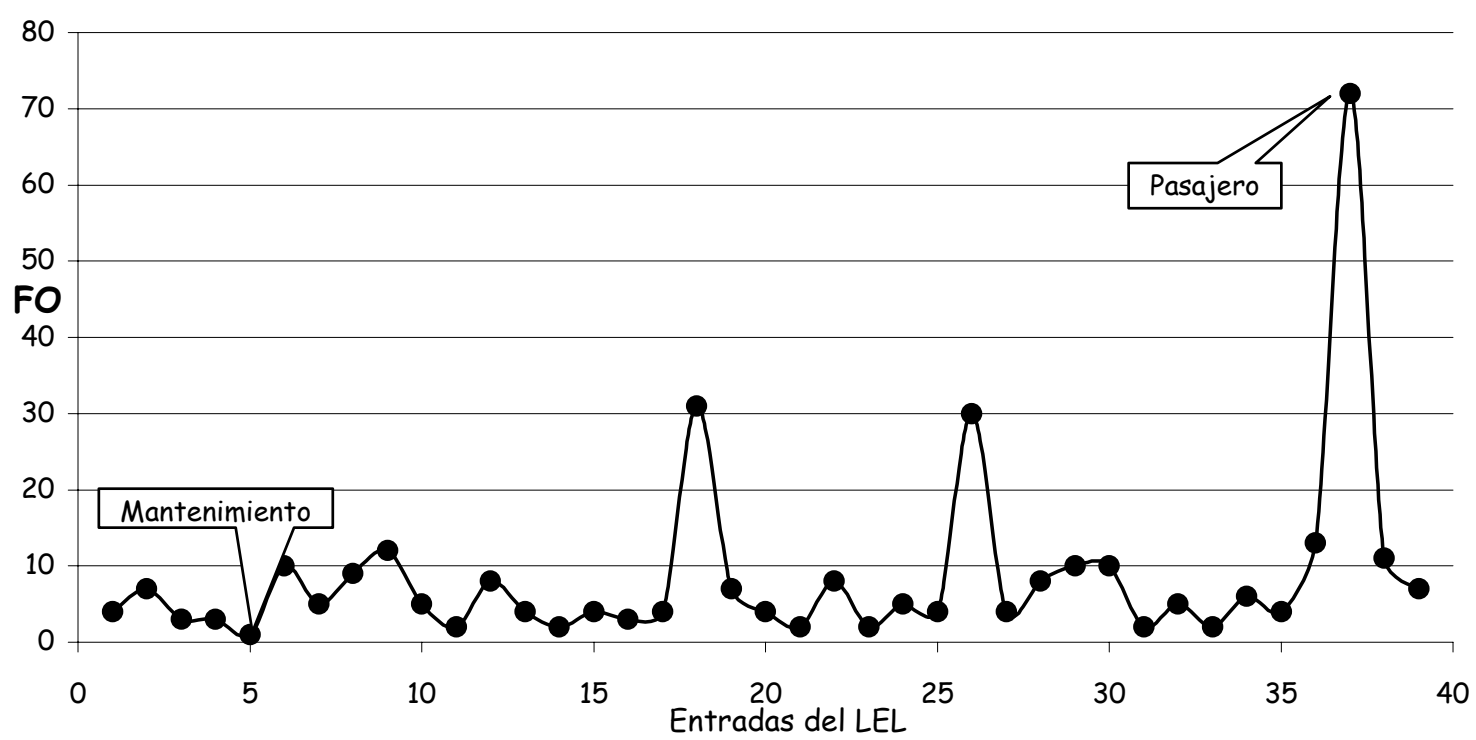

Figura VIII.9: FO de las entradas del LEL, ordenados por CPN creciente

\subsubsection{RELACIÓN ENTRE FAN OUT Y FAN IN}

Cada uno de los puntos que aparecen en la Figura VIII.10 representan el FO (en el eje de ordenadas) y el FI (en el eje de abcisas) de cada entrada del LEL en estudio.

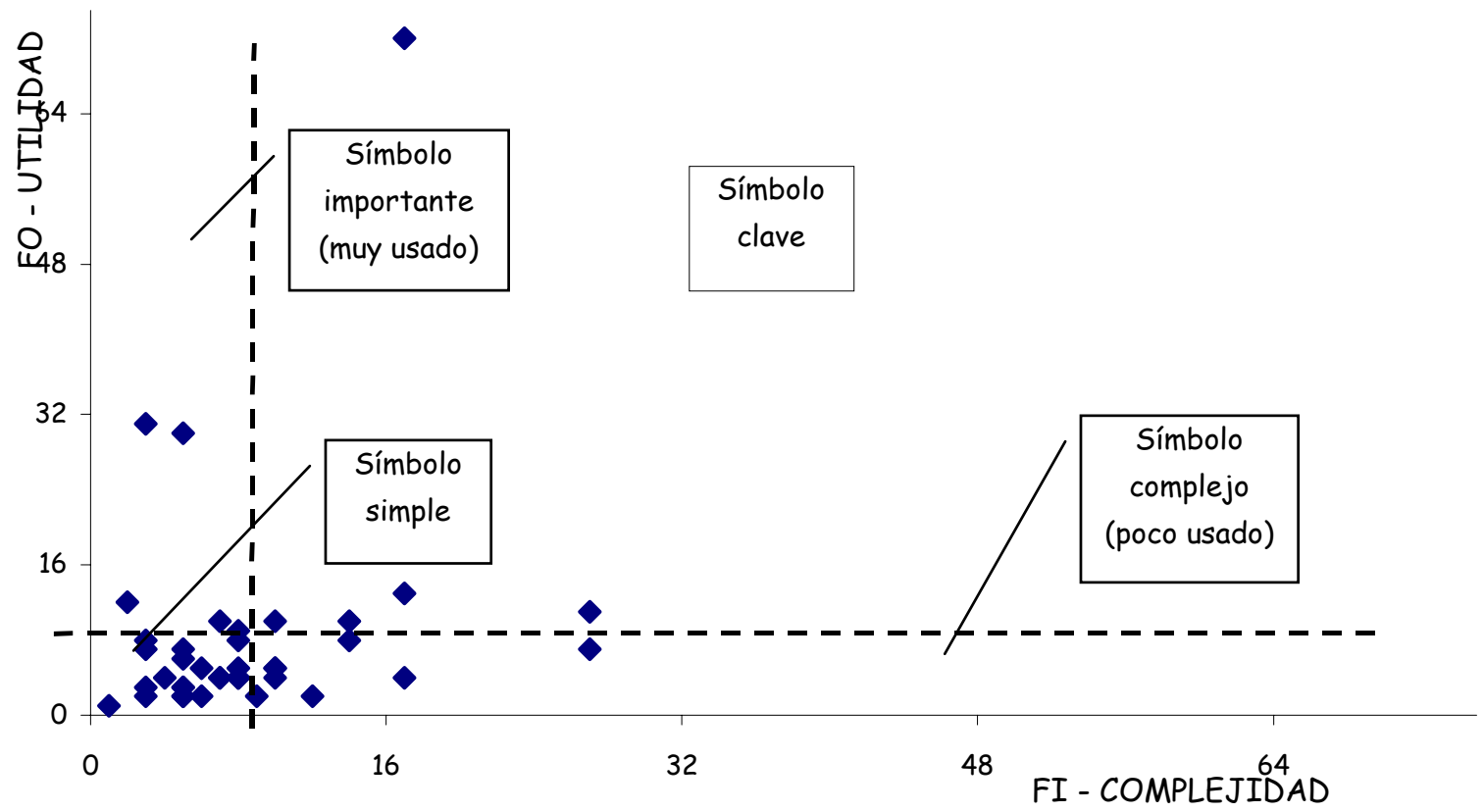

Figura VIII.10: FO vs. FI de las entradas del LEL 
Observando cómo se distribuyen los puntos en el gráfico, se ha visto que la mayor densidad corresponde al cuadrilátero delimitado por los valores 15 para cada uno de los ejes. Las líneas punteadas indican estos límites.

El eje correspondiente al FO, eje de ordenadas, representa la utilidad de un símbolo, cuanto más usado es un símbolo para definir otros símbolos, mayor será su FO y por lo tanto más útil es en el UdD.

El eje de abcisas en donde se representa el FI o CS está relacionado con la complejidad de un símbolo. Se dice que un símbolo que utiliza una gran cantidad de otros símbolos en su definición, es decir cuyo FI es elevado, es un símbolo complejo que representa conceptos importantes para el sistema, además de ser un indicador del buen uso del principio de circularidad.

La mayoría de los símbolos se agrupan en el cuadrante inferior izquierdo de la Figura VIII.10, ellos ostentan bajos valores de FO y FI. Son los símbolos simples y se debe prestar especial atención pues algunos de ellos, los de menor FO y FI, es decir los menos usados y menos complejos, pueden ser candidatos a ser redefinidos 0 eliminados.

El caso de Fan In alto respecto al Fan Out, incluye los símbolos que ocupan en la Figura VIII.10 el cuadrante inferior derecho. CHECK IN $(27,7), \operatorname{CHECK}$ OUT $(17,4)$, SOLICITUD DE RESERVA $(17,13)$ y RECEPCIONISTA $(27,11)$ son símbolos complejos ya que utilizan muchos símbolos en su definición en relación a la cantidad de símbolos que los utilizan. Estos conceptos, son de indudable importancia en un ambiente como un Hotel.

En este caso de estudio, el conocimiento del dominio sugiere que RECEPCIÓN $(3,31)$ es un símbolo de importancia central. Su Fan Out es 31 , es decir fue usado 31 veces para definir otros símbolos y utiliza en su definición sólo 3 símbolos. Esta situación lo ubica en la Figura VIII.10 en el cuadrante superior izquierdo como un símbolo importante. Una situación similar se da para HABITACIÓN $(5,30)$.

También están los símbolos clave en el dominio en estudio, son los que tienen tanto el Fan Out como el Fan In alto y ocupan el cuadrante superior derecho de la Figura VIII.10. Como ejemplo de esta clase de símbolos está PASAJERO/HUÉSPED/PAX $(17,72)$.

Una relación entre el Fan In y Fan Out que surge de un análisis similar al presentado en los párrafos precedentes lleva a establecer en la Definición 13 el coeficiente denominado FOI que se calcula como $F O(x) / F I(x)$ y se orienta, en primera instancia a medir la utilización de un símbolo, es decir su utilidad para definir otros símbolos y en un examen posterior indica su complejidad.

Se asume que un FOI alto indica un símbolo muy usado en el UdD en relación a la cantidad de entradas que usa en su definición y por lo tanto se podría decir que su utilidad es considerable. Mientras que un FOI bajo indica un símbolo altamente descriptivo que en su definición utiliza muchos símbolos y por lo tanto es importante para el ambiente que se modela y complejo en cuanto a su constitución.

En la Figura VIII.11 se presentan los datos obtenidos al calcular la relación FOI $(x)$. El $80 \%$ de los datos queda acotado en una franja que está entre $F O I(x)=0,2$ y $\operatorname{FOI}(x)=2$. Las excepciones son valores muy elevados como el caso de FOI (RECEPCIÓN) = 10,33 y lOS FOI(ADMINISTRACIÓN) y FOI(HABITACIÓN) que toman un valor 6 y otro muy bajo FOI(LISTA DE PRECIOS) de 0,17 


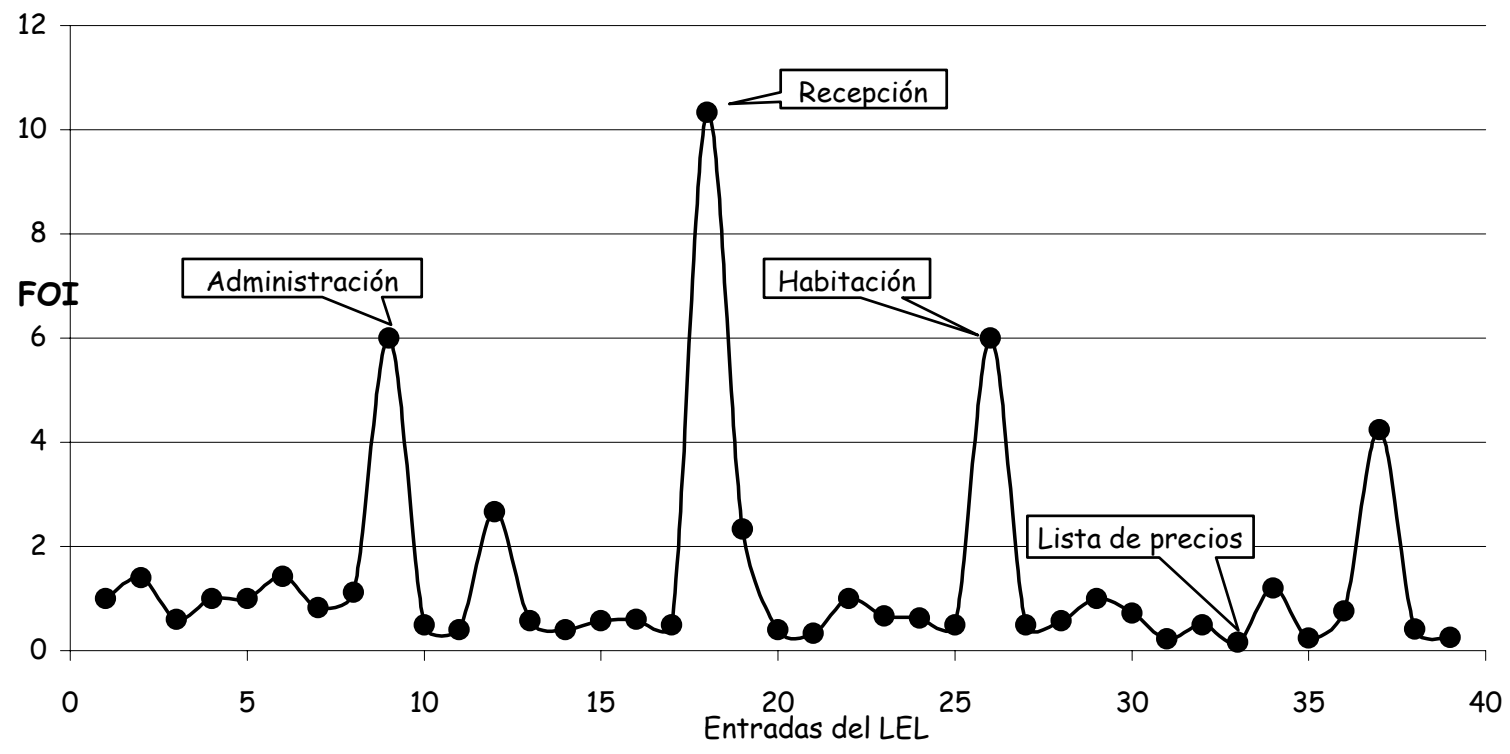

Figura VIII.11: FOI de las entradas del LEL, ordenados por CPN creciente

\subsubsection{TASA DE USO}

La Definición 14 establece que la Tasa de uso (TU) de un símbolo está dada por la relación entre el Fan Out del símbolo y la cantidad total de símbolos definidos (CSL) y se calcula con $F O(x)$ / CSL(L). La TU determina la relación entre la cantidad de veces que una entrada se usa para definir otras entradas y la cantidad de símbolos del LEL.

Una tasa de uso alta indica que se trata de un símbolo muy usado en el LEL para definir otros símbolos.

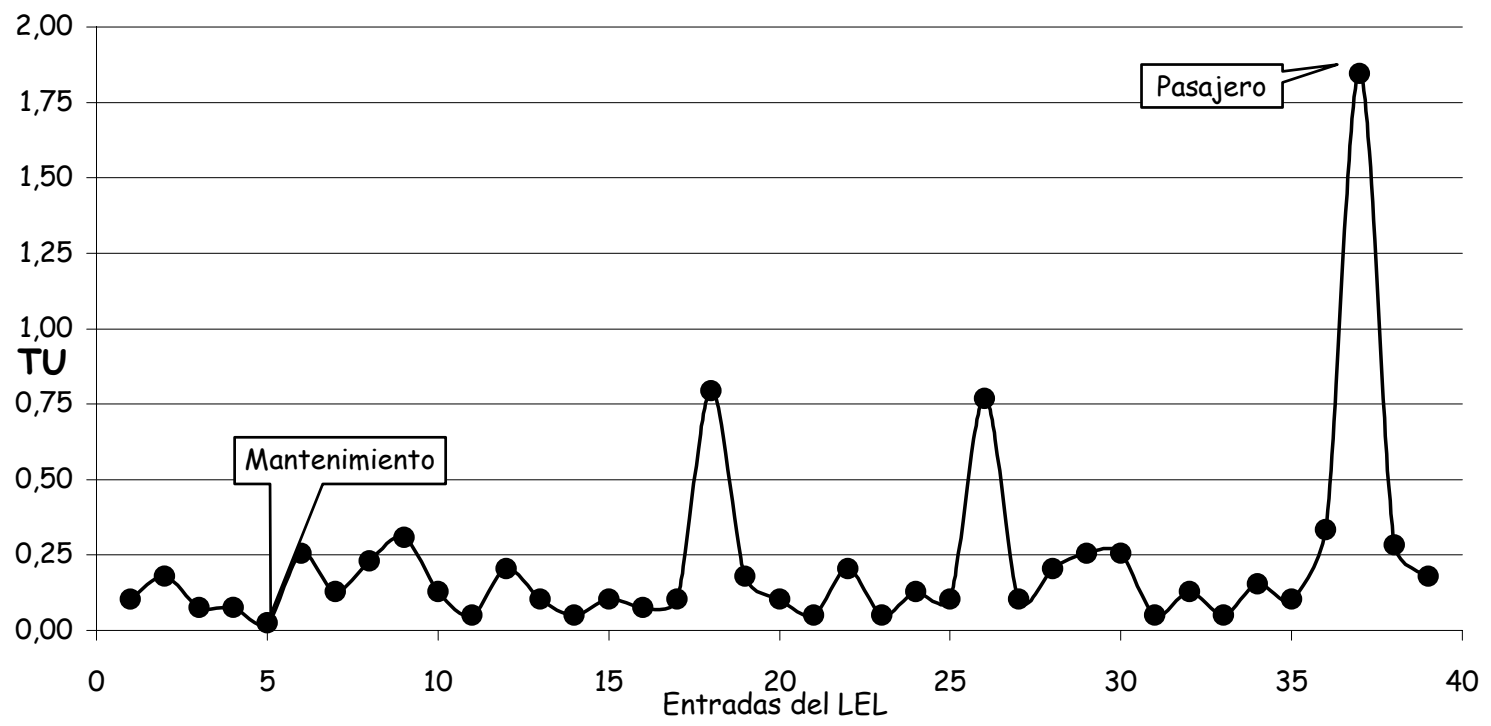

Figura VIII.12: TU de las entradas del LEL, ordenados por CPN creciente

En el LEL "Hotel" la tasa de uso tiene valores extremos en $T U($ MANTENIMIENTO $)=0,03$ evidenciando este valor que la entrada MANTENIMIENTO 
es poco usada para definir otras entradas y TU(PASAJERO/HUÉSPED/PAX) $=1,85$ valor que indica que esta es una entrada muy usada.

El $80 \%$ de las entradas toma valores que están entre $\operatorname{TU}(x)=0,25$ y $\operatorname{TU}(x)=$ 0,05

Si se pretendiera comparar los valores de distintas figuras, se debe tener cuidado ya que las magnitudes que se grafican difieren en un amplio rango y por lo tanto no se ha mantenido la misma escala en los gráficos.

\section{MEdiciones APLicadas a las Palabras del LEL ${ }^{2}$}

Se considerarán las mediciones registradas para cada símbolo del LEL correspondientes a: CVN (cantidad de verbos normalizados) que sumadas dan CVNL y representa todas las veces que se usaron verbos normalizados para definir las entradas del LEL; CS (cantidad de símbolos) que sumados dan CSUL y que representa las veces que se usaron símbolos del LEL en la definición de otros símbolos y COPN (cantidad de otras palabras normalizadas) cuya suma representa la cantidad de cualquier otra palabra normalizada que no es ni verbo ni entrada del LEL y que fue usada en la definición de las entradas del LEL, asi se obtiene COPNL. La suma de CVNL, CSUL y COPNL da CPNL que es la cantidad total de palabras normalizadas usadas para definir, en este caso, el LEL "Hotel". Cabe recordar que en estas mediciones se contabilizan todas las palabras normalizadas, aún aquéllas que estuvieran repetidas.

Las particularidades de las Definiciones utilizadas en este apartado se pueden consultar en el Capítulo VII.

\subsection{MEDICIONES INMEDIATAS}

\subsubsection{VALORES TOtALES}

\begin{tabular}{|l|c|}
\hline CSL(Hotel) & 39 \\
\hline CPL (Hotel) & 2616 \\
\hline CPNL(Hotel) & 1035 \\
\hline CVNL(Hotel) & 230 \\
\hline CSUL(Hotel) & 332 \\
\hline COPNL(Hotel) & 473 \\
\hline
\end{tabular}

Los valores totales de la Tabla VIII.5 se basan en las Definiciones 1 a 7 indicadas en el Capítulo VII y se obtuvieron sumando los atributos correspondientes a cada símbolo $x_{i}$ del LEL "Hotel".

Tabla VIII.5: Valores totales

Estas fórmulas expresan la cantidad de veces que se usaron las mediciones indicadas para definir todas las entradas del LEL.

Cantidad de palabras del LEL:

$$
C P L(\text { Hotel })=\sum_{i=1}^{C S L(\text { Hotel) }} C P\left(x_{i}\right)
$$

\footnotetext{
${ }^{2}$ La Tabla 2 del Apéndice F contiene las mediciones efectuadas al LEL
} 
Cantidad de palabras normalizadas del LEL:

$$
\operatorname{CPNL}(\text { Hotel })=\sum_{i=1}^{C S L(\text { Hotel) }} \operatorname{CPN}\left(x_{i}\right)
$$

Cantidad de verbos normalizados del LEL:

$$
\text { CVNL }(\text { Hotel })=\sum_{i=1}^{\operatorname{csL}(\text { Hotel) }} \operatorname{CVN}\left(x_{i}\right)
$$

Cantidad de símbolos del LEL:

$$
\operatorname{CSUL}(\text { Hotel })=\sum_{i=1}^{C S L(\text { Hotel) }} C S\left(x_{i}\right)
$$

Cantidad de otras palabras normalizadas del LEL:

$$
\operatorname{COPNL}(\text { Hotel })=\text { CPNL }(\text { Hotel })-(\text { CVNL }(\text { Hotel })+\text { CSUL }(\text { Hotel }))
$$

La cantidad total de palabras normalizadas usadas para elaborar todas las definiciones del LEL, incluyen los símbolos y los verbos que se contabilizan en forma inmediata, pero existe una diferencia que corresponde a lo que se definió para cada entrada como COPN. Para el LEL considerado en su conjunto, este valor se indica con COPNL.

En la Figura VIII.13 se muestra la distribución en porcentaje de los componentes COPNL, CVNL y CSUL de los símbolos del LEL "Hotel" considerados en su conjunto.

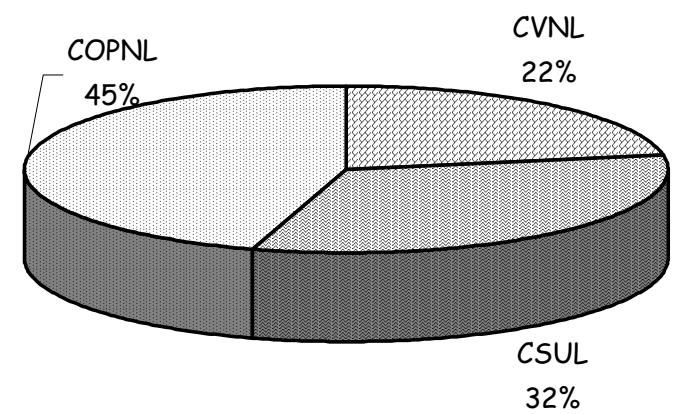

Figura VIII.13: Contribución de cada tipo de palabra normalizada del LEL "Hotel"

El mayor valor $45 \%$ pertenece a las palabras que no son ni verbos ni símbolos del LEL: COPNL. Comparando estos porcentajes, con los valores obtenidos en la Tabla 1 del Apéndice F cuando se analizó cada entrada, se observa que 21 entradas tienen un porcentaje de COPN mayor que el $45 \%$ y 18 tienen un porcentaje menor, es decir el porcentaje hallado para la COPNL corresponde a un valor promedio para las entradas individuales.

Para la CVNL se obtiene un $22 \%$, al remitirse a la Tabla 1 del Apéndice F se pueden contabilizar 19 entradas con un porcentaje mayor y 20 con un porcentaje menor o igual. Algo similar ocurre con la CSUL que registra para todas las entradas un $32 \%$, haciendo un análisis individual a partir de la Tabla 1 del Apéndice F se llega a que 21 entradas tienen un porcentaje mayor y 18 tienen un porcentaje menor o igual.

Esto significa que en una primera aproximación los porcentajes individuales se reparten en forma equitativa a partir de los porcentajes generales obtenidos para todo el LEL. Es decir la Figura VIII.13 representa valores que pueden considerarse una lectura intermedia para cada grupo de palabras. 


\subsubsection{DISTRIBUCIÓN DE LOS SÍMBOLOS DEL LEL SEGÚN LA CLASIFICACIÓN}

Los símbolos se agruparon según la clasificación adoptada en función de las actividades del sistema en estudio, pero con el objeto de tender a la generalización, se prefirió usar en este análisis la clasificación dada en [Hadad '97], que establece las categorías Verbo, Objeto y Sujeto. La Tabla VIII.6 consigna los valores obtenidos. Esta medición se indica como DSLC(nombre del LEL, grupo de pertenencia).

\begin{tabular}{|c|c|}
\hline CLASIFICACIÓN GENERAL & DSLC \\
\hline$\checkmark$ Verbo & 12 \\
\hline$\checkmark$ Objeto & 25 \\
\hline$\checkmark$ Sujeto & 2 \\
\hline TOTAL & 39 \\
\hline
\end{tabular}

Tabla VIII.6: Clasificación de las entradas del LEL "Hotel"

\subsection{MEDICIONES CALCULADAS}

Para el análisis de los resultados obtenidos aplicando mediciones estadísticas a los datos se trabajará con todas las entradas del LEL debido a que su tamaño es pequeño, 39 símbolos, no es necesario tomar una muestra. Se lo denominará población - LEL. También se evaluarán los datos dentro del grupo al que pertenecen según la clasificación general. A cada uno de estos grupos se los llamará categorías o subpoblaciones.

Todos los cálculos que figuran en esta sección se efectuaron utilizando las fórmulas estadísticas que ofrece la planilla de cálculo Excel 2000 de Microsoft.

\subsubsection{MEDICIONES DE TENDENCIA CENTRAL}

La tendencia central se refiere al punto medio de una distribución, estas medidas se conocen también como medidas de posición.

La media o promedio es el resultado de sumar todos los datos y dividirlos por la cantidad de observaciones. Si la población contiene mediciones homogéneas, indica un valor del parámetro analizado que caracteriza al conjunto.

Al ordenar los datos en forma creciente o decreciente, se puede identificar la mediana que es la observación que ocupa el lugar central en el conjunto, de forma tal que el $50 \%$ de las observaciones serán mayores que este valor y el otro $50 \%$ será menor.

La observación cuya frecuencia es más alta en el conjunto de datos, es decir el valor que más veces se repite, se la denomina moda.

Cuando los valores registrados para la media, la mediana y la moda coinciden se está en presencia de una distribución normal.

Si en un conjunto de mediciones se registran valores extremos, la media no servirá para caracterizar al conjunto de los datos, pues su valor queda desplazado por 
la influencia de esas mediciones, en este caso la mediana será una medida más representativa de la población que la media.

El cálculo de los extremos superior e inferior de un conjunto de datos permite determinar valores inusualmente altos o bajos que desdibujan el valor de la media. En la Tabla VIII.7 se consignan los resultados del cálculo de los valores necesarios para individualizar los valores extremos para la cantidad de palabras normalizadas.

\begin{tabular}{|l|c|c|c|c|c|c|c|c|}
\cline { 2 - 8 } \multicolumn{1}{c|}{} & $Q_{m}$ & $Q_{i}$ & $Q_{s}$ & Long & Extr. I & Extr. S & Val. I & Val. S \\
\hline CPNL & 23 & 18 & 30 & 12 & 12 & 48 & $11 ; 11$ & $49 ; 50 ; 51 ; 75$ \\
\hline
\end{tabular}

Tabla VIII.7: Determinación de valores extremos para CPNL

$Q_{m}$ corresponde al valor de la mediana, $Q_{i}$ y $Q_{s}$ corresponden a los valores de la mediana de la mediana inferior y superior respectivamente. La longitud (Long) de una hipotética caja que contiene los datos centrales registrados para la población en estudio, se calcula como la diferencia entre $Q_{s}$ y $Q_{i}$. Luego se determinan los extremos inferior (Extr. I) y superior (Extr. S) de la distribución en función de la longitud de la caja y el $Q_{s}$ utilizando las fórmulas indicadas en el parágrafo 1.4 Cuartiles del Apéndice D. Estos puntos representan los límites teóricos entre los cuales se esperaría encontrar todos los datos si la distribución fuera normal.

Los valores que quedan fuera de una distribución normal para la cantidad de palabras normalizadas en el LEL "Hotel" corresponden para los valores inferiores (Val. I) a las entradas FICHERO DE PASAJEROS (11), TARIFA (11) y para los valores superiores (Val. S) SOLICITUD DE RESERVA (49), PASAJERO /HUÉSPED /PAX (50), RECEPCIONISTA (51) Y CHECK IN (75). De alguna manera estas entradas son excepcionales en este LEL Y corresponden al $15 \%$ de las entradas. Habría que determinar si este porcentaje puede desecharse a la hora de caracterizar un LEL.

En la Tabla VIII.8 se realiza un análisis similar pero para las clases de palabras que constituyen la cantidad de palabras normalizadas del LEL, CPNL.

\begin{tabular}{|l|c|c|c|c|c|c|c|c|}
\cline { 2 - 9 } \multicolumn{1}{c|}{} & $Q_{m}$ & $Q_{i}$ & $Q_{s}$ & Long & Extr. I & Extr. S & Val. I & Val. S \\
\hline CVNL & 6 & 4 & 7 & 3 & 2,5 & 11,5 & $2 ; 2 ; 2$ & $12 ; 14 ; 15$ \\
\hline CSUL & 7 & 5 & 10 & 5 & 2,5 & 17,5 & $1 ; 2$ & $27 ; 27$ \\
\hline COPNL & 11 & 8 & 15 & 7 & 4,5 & 25,5 & $3 ; 3 ; 3 ; 4$ & 33 \\
\hline
\end{tabular}

Tabla VIII.8: Determinación de valores extremos para las clases de palabras normalizadas

La medición correspondiente a CSUL, la cantidad de símbolos usados en las definiciones, es la que presenta una menor cantidad de valores extremos, son sólo 4 y por lo tanto es un conjunto más homogéneo en cuanto a las mediciones registradas.

Este análisis llevaría a pensar que no considerando los valores extremos en los cálculos estadísticos, se tendrían mediciones de la tendencia central más representativas de la población. 
En la Tabla VIII.9 figuran los resultados obtenidos al calcular las mediciones de la tendencia central sin incluir los valores extremos en comparación a los obtenidos al incluirlos.

\begin{tabular}{c|c|c|c|c|}
\hline & \multicolumn{3}{|c|}{ SIN VALORES EXTREMOS } \\
\cline { 2 - 5 } & CPNL & CVNL & CSUL & COPNL \\
\cline { 2 - 5 } & 23,88 & 5,55 & 7,86 & 12.56 \\
\cline { 2 - 5 } & 23 & 6 & 7 & 11,5 \\
\hline 23 & 6 & 5 & 8 \\
\hline
\end{tabular}

(a)

\begin{tabular}{c|c|c|c|c|} 
& \multicolumn{4}{|c|}{ CON VALORES EXTREMOS } \\
\cline { 2 - 5 } & CPNL & CVNL & CSUL & COPNL \\
\cline { 2 - 5 } MEDIA & 26,54 & 5,90 & 8,51 & 12,13 \\
\hline MEDIANA & 23 & 6 & 7 & 11 \\
\cline { 2 - 5 } MODA & 23 & 6 & 5 & 8 \\
\hline
\end{tabular}

(b)

Tabla VIII.9: Comparación de mediciones de la tendencia central para el LEL "Hotel" sin valores extremos (a) y con valores extremos (b)

Tal cual como podría esperarse, la moda no se ve afectada por los valores extremos, ya que no depende de ellos pues es el valor de mayor frecuencia.

La mediana, debería desplazarse ya que su valor depende de la cantidad de observaciones. En el caso de la CVNL se excluyen 6 mediciones extremas, en el de COPNL 5 y en el de CSUL 4, sin embargo en ninguno de ellos se ve sensiblemente afectado el resultado.

La media es la medición que resulta más sensible a la eliminación de los casos extremos. El valor más afectado corresponde al caso de la CSUL, se produce una disminución de su valor de 8,51 a 7,86 lo que representa un 7,64\%. Aún en este caso, el más sensible, la variación es mínima.

En la Tabla VIII.9 queda de manifiesto que los valores extremos no tienen demasiada influencia en los resultados obtenidos para las mediciones de la tendencia media de este LEL. Por lo tanto se considerarán todos las entradas para los cálculos que se presenten a continuación.

Ahora se analizará la forma de la distribución en cada tabla para cada clase de palabra. Es interesante el comportamiento que se observa para la CPNL, eliminando los valores extremos se estaría en presencia de una distribución normal pues moda y mediana coinciden y la media toma un valor cuya diferencia con la moda y la mediana es mínima: 0,88. Incluyendo los valores extremos, se obtiene para la media 26,54 mientras que moda y mediana se mantienen en el valor 23. Aquí ya no sería posible hablar estrictamente de una distribución normal, pero de igual forma estaría muy cerca de serlo ya que la diferencia es del 15,39\%

Este análisis demuestra que en el LEL "Hotel" la distribución de las observaciones de la cantidad de verbos normalizados no está influenciada por las observaciones extremas y responde a una distribución normal. Incluyendo todas las observaciones, la media, la mediana y la moda coinciden en el valor 6 (se considera que el valor 5,90 es suficientemente aproximados a 6 como para considerarlos iguales) y sin tomar en cuenta las mediciones extremas, los valores registrados son 5,55; 6 y 6 , estos valores tienen una diferencia de menos del $10 \%$ entre ellos.

Las distribuciones de la cantidad de símbolos y la cantidad de otras palabras normalizadas no es normal. Específicamente la mayor variación la presenta la COPNL, esto corrobora la conclusión a la que se arribó por la observación que se hizo de las 
Figuras VIII.2 y VIII.3 respecto a la alta variación de los valores de COPN y a la mayor estabilidad de los valores de CVN.

También resulta interesante el comportamiento de las mediciones propuestas en el entorno de cada subpoblación o categoría de entradas que se definieron en el parágrafo 2.1 .2 de este mismo capítulo, como Verbo, Objeto y Sujeto. Estos valores se consignan en la Tabla VIII.10

\begin{tabular}{c|c|c|c|c|c|}
\cline { 2 - 6 } ENTRADAS & CPNL & CVNL & CSUL & COPNL & MEDICIONES \\
\hline \multirow{4}{*}{ VERBO } & 32,08 & 7,08 & 10,83 & 14,17 & MEDIA \\
& 23,50 & 6 & 9,5 & 11,5 & MEDIANA \\
& 21 & 6 & 10 & 8 & MODA \\
\hline \multirow{3}{*}{ OBJETO } & 21,96 & 4,88 & 6,46 & 10,63 & MEDIA \\
& 21 & 5 & 6,5 & 9 & MEDIANA \\
& 14 & 3 & 8 & 9 & MODA \\
\hline \multirow{3}{*}{ SUJETO } & 50,50 & 13 & 22 & 15,5 & MEDIA \\
& 50,50 & 13 & 22 & 16 & MEDIANA \\
& $\#$ N/A & $\#$ N/A & $\#$ N/A & $\#$ N/A & MODA \\
\hline \multirow{5}{*}{ LEL } & 26,54 & 5,90 & 8,51 & 12,13 & MEDIA \\
& 23 & 6 & 7 & 11 & MEDIANA \\
& 23 & 6 & 5 & 8 & MODA \\
\hline
\end{tabular}

Tabla VIII.10: Mediciones de la tendencia central para las categorías Verbo, Objeto y Sujeto

Para la categoría Sujeto los valores difieren enormemente del resto. Esto se debe a que comprende sólo dos símbolos que utilizaron en su definición una cantidad de palabras normalizadas mucho mayor que el resto de las definiciones, RECEPCIONISTA con 51 y PASAJERO con 50 , sólo superadas por la entrada CHECK IN con 75 palabras normalizadas.

En cuanto a las mediciones de tendencia central registradas para las categorías Verbo y Objeto no difieren mucho de las correspondientes para la población, según puede apreciarse en la Tabla VIII.10

Los valores registrados para cada clase de palabra y para cada categoría de la media, mediana y moda difieren entre sí pero en cantidades que se pueden considerar ínfimas. Entonces se podría decir que la distribución está muy cercana a ser normal.

\subsubsection{MEDICIONES DE DISPERSIÓN}

La dispersión se refiere a la extensión de los datos en una distribución, es decir, el grado en que las observaciones se distribuyen. Es una característica que permite juzgar la confiabilidad de las medidas de tendencia central. Si los datos se encuentran ampliamente dispersos, la posición central es menos representativa de la población que cuando se encuentran estrechamente agrupados alrededor de la media.

El desvío estándar evalúa la distancia de cada una de las lectura hechas en relación a la media de la población, por lo tanto cuanto mayor sea su valor, significará

\footnotetext{
${ }^{3}$ No se puede calcular la moda para el grupo Sujeto porque no hay valores que se repitan
} 
que las mediciones se alejan de la media calculada y por lo tanto la media no será un parámetro representativo del conjunto.

El coeficiente de variación es el cociente entre el desvío estándar y la media de una población dada, expresa el grado de dispersión de los datos con respecto a la media. Es una medida relativa que permite establecer comparaciones entre poblaciones con distintos valores de la media aritmética. Cuanto más alejado esté su valor de 1 significa que los datos están más dispersos.

\begin{tabular}{c|c|c|c|c|c|}
\cline { 2 - 5 } & \multicolumn{4}{|c|}{ CLASES DE PALABRAS } & \multicolumn{1}{c}{} \\
\hline ENTRADAS & CPNL & CVNL & CSUL & COPNL & MEDICIONES \\
\hline \multirow{2}{*}{ VERBO } & 16,36 & 2,93 & 6,58 & 7,98 & DESV ST \\
& 0,51 & 0.41 & 0.61 & 0,56 & COEF. VARIACIÓN \\
\hline \multirow{2}{*}{ OBJETO } & 7,88 & 1,83 & 3,10 & 5,52 & DESV ST \\
& 0,36 & 0,38 & 0,48 & 0.52 & COEF. VARIACIÓN \\
\hline \multirow{2}{*}{ SUJETO } & 0,5 & 1 & 5 & 5,5 & DESV ST \\
& 0,01 & 0,08 & 0,23 & 0,35 & COEF. VARIACIÓN \\
\hline \multirow{2}{*}{ LEL } & 13,15 & 2,97 & 5,9 & 6,61 & DESV ST \\
& 0,50 & 0,50 & 0,69 & 0,55 & COEF. VARIACIÓN \\
\hline
\end{tabular}

Tabla VIII.11: Mediciones de la dispersión para las categorías y el LEL

A partir de la información que figuran en la Tabla VIII.11 se puede concluir que los datos para las categorías establecidas o subpoblaciones y para el LEL o población no presentan un grado alto de dispersión pues los coeficientes de variación son todos menores que 1 y por lo tanto la media es una medición representativa de la población.

El Teorema de Chebyshev establece que independientemente de la forma que tenga una distribución, al menos el $75 \%$ de los valores caen dentro de \pm 2 desviaciones estándar a partir de la media de la distribución y que al menos el $89 \%$ caen dentro de \pm 3 desviaciones estándar a partir de la media de la población.

La aplicación de este teorema a los datos del LEL "Hotel", origina la Tabla VIII.12. Para obtener el límite superior del rango, se ha sumado a la media el desvío estándar multiplicado por dos y para obtener el límite inferior se ha realizado una resta de las mismas cantidades.

Se obtienen valores del extremo inferior del intervalo que son negativos y por lo tanto imposibles para una cantidad de palabras. El valor 0 si bien no es imposible, es muy difícil. Las cantidades negativas se llevan a 0 y los valores decimales (imposibles cuando se están midiendo palabras) se redondearon al entero más próximo. Se incluyen las mediciones menores e iguales y mayores e iguales que los extremos de los rangos calculados.

La cantidad de entradas que cumplen con los rangos calculados por cada clase de palabra y por categoría se obtienen de la Tabla 2 del Apéndice F. En la Tabla VIII.12, en la fila titulada CANTIDAD se consigna la cantidad de entradas de cada categoría de palabras que están dentro del rango calculado. En la columna con el encabezamiento $75 \%$ se indica la cantidad de entradas que corresponden a ese porcentaje en este LEL. 
Al contar en la Tabla 2 del Apéndice $F$ la cantidad de entradas incluidas en los rangos calculados para cada categoría de entrada y para el LEL y dentro de ellas para cada clase de palabra, se puede determinar que la cantidad de observaciones que caen en el rango especificado es bastante mayor que el $75 \%$ esperados según el Teorema de Chebyshev. Esto llevaría a pensar que la distribución de esta población se ajusta a parámetros más estrictos. Por otro lado, en el parágrafo 2.2.1 se llegó a la conclusión de que la distribución de las subpoblaciones y de la población podrían considerarse cercanas a ser normales. Por todo lo anterior, se podría asegurar que, efectivamente, se estaría frente a una distribución normal tanto de las categorías de entradas como del LEL.

\begin{tabular}{|c|c|c|c|c|c|c|}
\cline { 2 - 5 } \multicolumn{1}{c|}{} & \multicolumn{3}{c|}{ CLASES DE PALABRAS } & \multicolumn{1}{c|}{} \\
\hline ENTRADAS & CPNL & CVNL & CSUL & COPNL & MEDICIONES & $75 \%$ \\
\hline \multirow{4}{*}{ VERBO } & 32,08 & 7,08 & 10,83 & 14,17 & MEDIA & \\
& 16,36 & 2,93 & 6,58 & 7,8 & DESV ST & \\
& $0 ; 65$ & $1 ; 13$ & $0 ; 24$ & $0 ; 30$ & RANGO & \\
& 11 & 11 & 11 & 11 & CANTIDAD & 9 \\
\hline \multirow{5}{*}{ OBJETO } & 21,96 & 4,88 & 6,46 & 10,63 & MEDIA & \\
& 7,88 & 1,83 & 3,10 & 5,52 & DESV ST & \\
& $6 ; 38$ & $1 ; 10$ & $0 ; 12$ & $0 ; 22$ & RANGO & \\
& 25 & 25 & 24 & 24 & CANTIDAD & 19 \\
\hline \multirow{5}{*}{ SUJETO } & 50,5 & 13 & 22 & 15,5 & MEDIA & \\
& 0,5 & 1 & 5 & 5,5 & DESV ST & \\
& $50 ; 52$ & $11 ; 15$ & $12 ; 32$ & $5 ; 27$ & RANGO & \\
& 2 & 2 & 2 & 2 & CANTIDAD & 1 \\
\hline \multirow{5}{*}{ LEL } & 26.54 & 5,9 & 8,51 & 12,13 & MEDIA & \\
& 13,15 & 2,97 & 5,90 & 6,61 & DESV ST & \\
& $0 ; 53$ & $0 ; 12$ & $0 ; 20$ & $0 ; 25$ & RANGO & \\
& 38 & 37 & 37 & 37 & CANTIDAD & 29 \\
\hline
\end{tabular}

Tabla VIII.12: Determinación del rango de valores del 75\% de las mediciones según el Teorema de Chebyshev

Siempre que se esté en presencia de una distribución normal, se puede anticipar que aproximadamente el $68 \%$ de todos los valores se encuentra dentro de \pm 1 desvío estándar de la media.

Se hará el mismo tipo de análisis efectuado para armar la Tabla VIII.12 pero suponiendo ahora que las distribuciones son normales, así se obtiene la Tabla VIII.13

En ningún caso se registra en la Tabla VIII.13 una cantidad de entradas menor que el $68 \%$ previsto para una distribución normal, muy por el contrario siempre son mayores con la excepción de la CPNL para la categoría Sujeto que es igual.

En resumen, se puede afirmar que el $68 \%$ de las mediciones efectuadas a la cantidad de palabras normalizados de este LEL estarán en los rangos especificados en la Tabla VIII.13 


\begin{tabular}{|c|c|c|c|c|c|c|}
\cline { 2 - 5 } \multicolumn{1}{c|}{} & \multicolumn{3}{c|}{ CLASES DE PALABRAS } & \multicolumn{1}{c|}{} \\
\hline ENTRADAS & CPNL & CVNL & CSUL & COPNL & MEDICIONES & $68 \%$ \\
\hline \multirow{4}{*}{ VERBO } & 32,08 & 7,08 & 10,83 & 14,17 & MEDIA & \\
& 16,36 & 2,93 & 6,58 & 7,98 & DESV ST & \\
& $16 ; 48$ & $4 ; 10$ & $4 ; 18$ & $6 ; 22$ & RANGO & \\
& 10 & 11 & 10 & 10 & CANTIDAD & 8 \\
\hline \multirow{5}{*}{ OBJETO } & 21,96 & 4,88 & 6,46 & 10,63 & MEDIA & \\
& 7,88 & 1,83 & 3,10 & 5,52 & DESV ST & \\
& $14 ; 30$ & $3 ; 7$ & $3 ; 9$ & $5 ; 17$ & RANGO & \\
& 20 & 22 & 20 & 18 & CANTIDAD & 17 \\
\hline \multirow{5}{*}{ SUJETO } & 50,5 & 13 & 22 & 15,5 & MEDIA & \\
& 0,5 & 1 & 5 & 5,5 & DESV ST & \\
& $51 ; 52$ & $12 ; 14$ & $17 ; 27$ & 10,$5 ; 22$ & RANGO & \\
& 1 & 2 & 2 & 2 & CANTIDAD & 1 \\
\hline \multirow{5}{*}{ LEL } & 26.54 & 5,9 & 8,51 & 12,13 & MEDIA & \\
& 13,15 & 2,97 & 5,90 & 6,61 & DESV ST & \\
& $13 ; 40$ & $3 ; 9$ & $3 ; 14$ & $6 ; 19$ & RANGO & \\
& 33 & 33 & 35 & 30 & CANTIDAD & 27 \\
\hline
\end{tabular}

Tabla VIII.13: Determinación del rango de valores del 68\% de las mediciones según una distribución normal

Aún más, aproximadamente el $85 \%$ de las entradas del LEL "Hotel" se encuentran en los rangos previstos para una distribución normal, esto indica que las mediciones de la tendencia central (media, mediana y moda) son realmente representativas de la población.

Para todos los análisis estadísticos efectuados se observa que la categoría Sujeto exhibe valores atípicos con respecto a lo valores calculados para el LEL. El motivo es que esta categoría incluye sólo dos entradas y éstas son especiales pues sus mediciones dan valores mucho mayores que los del resto de las entradas, a excepción de la entrada CHECK IN.

Los valores consignados en la fila RANGO de la Tabla VIII.13 para el LEL coinciden con los registrados en el parágrafo 1.1 .3 para aproximadamente el $80 \%$ de las entradas analizadas en forma individual.

\subsubsection{ANÁLISIS DE CORRELACIÓN}

Se realizaron cuatro diagramas de dispersión usando la CPN como variable independiente y como variable dependiente: la CP (Figura VIII.14), la CS (Figura VIII.15), la CVN (Figura VIII.16) y la COPN (Figura VIII.17).

La tabla de valores usada para la realización de estos gráficos se ordenó en forma ascendente por la columna correspondiente a CPN.

En todos los diagramas se observa una mayor densidad de puntos para valores bajos de CPN. 
Se trata de trazar una recta uniendo la mayor cantidad posible de puntos o al menos pasando a la mínima distancia posible de ellos. En general los puntos se ajustan bastante bien a la recta propuesta que se denomina línea de regresión 4 .

Los datos graficados muestran una cierta tendencia, se puede inferir que podría existir alguna relación entre los ellos, pero no se puede asegurar que unos sean consecuencia de los otros.

El coeficiente de determinación es el cuadrado del coeficiente de correlación. Para explicar la variación de la variable dependiente en un conjunto de datos respecto a la línea de regresión y a la media se prefiere el coeficiente de determinación. Si la relación es lineal perfecta, el coeficiente será 1 , si no existe correlación alguna será 0 .

En la Tabla VIII.14 se sumarizan los coeficientes de determinación hallados para la cantidad de palabras que intervienen en una definición y para cada una de las clases de palabras con respecto a la cantidad de palabras normalizadas. Se toma a la cantidad de palabras normalizadas como la variable independiente, ya que es la medición básica de todo este trabajo.

$\begin{array}{lc}\text { Pares de datos } & \text { Coef. de determinación } \\ \text { CPN - CP } & 0,9105 \\ \text { CPN - CVN } & 0,7784 \\ \text { CPN - CS } & 0,7377 \\ \text { CPN - COPN } & 0,6817\end{array}$

Tabla VIII.14: Valores calculados para el coeficiente de determinación: $r^{2}$

A partir de los datos obtenidos se puede concluir que la relación más estrecha corresponde a la cantidad de palabras con la cantidad de palabras normalizadas, pues el valor es el más cercano a 1 que indica una correlación perfecta. La interpretación de este valor indica que el $91,05 \%$ de la variación de CP está explicada por la línea de regresión, es decir su grado de asociación con CPN. Hecho que resulta fácilmente observable a partir de la Figura VIII.14 en donde los puntos graficados se encuentran muy cerca de la línea de regresión.

\footnotetext{
${ }^{4}$ El programa Excel (Microsoft Office 2000) provee facilidades para el trazado de la línea de regresión y el cálculo del coeficiente de determinación
} 


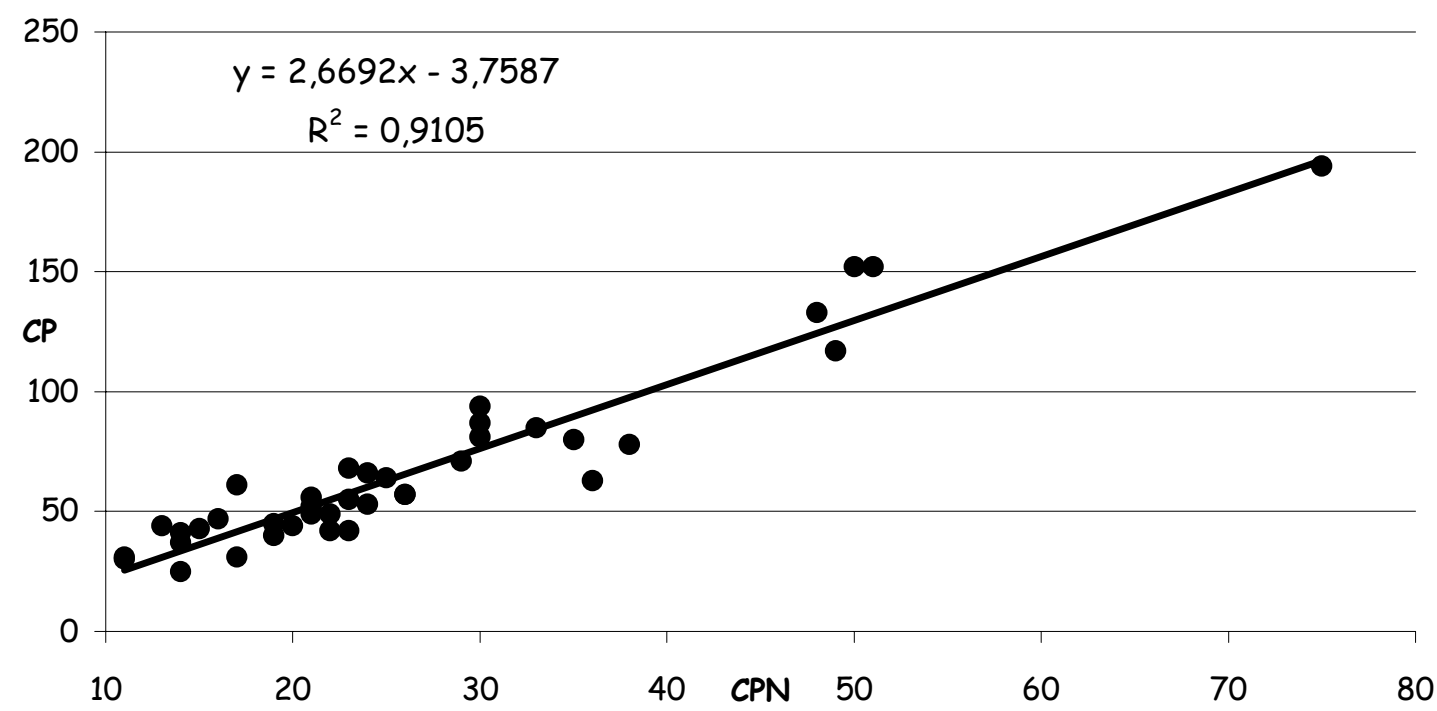

Figura VIII.14: Diagrama de dispersión y línea de regresión para CPN vs. CP

La cantidad de verbos normalizados está bastante fielmente representada por la línea de regresión, los datos graficados que se observan en la Figura VIII.15 no se encuentran muy alejados de ella. Por lo tanto se podría asegurar que la variación de la cantidad de verbos normalizados de una entrada tendría relación con la cantidad de palabras normalizadas de dicha entrada. Para ser más exacto, el $77,84 \%$ de la cantidad de verbos normalizados (CVN) usados está explicada por CPN.

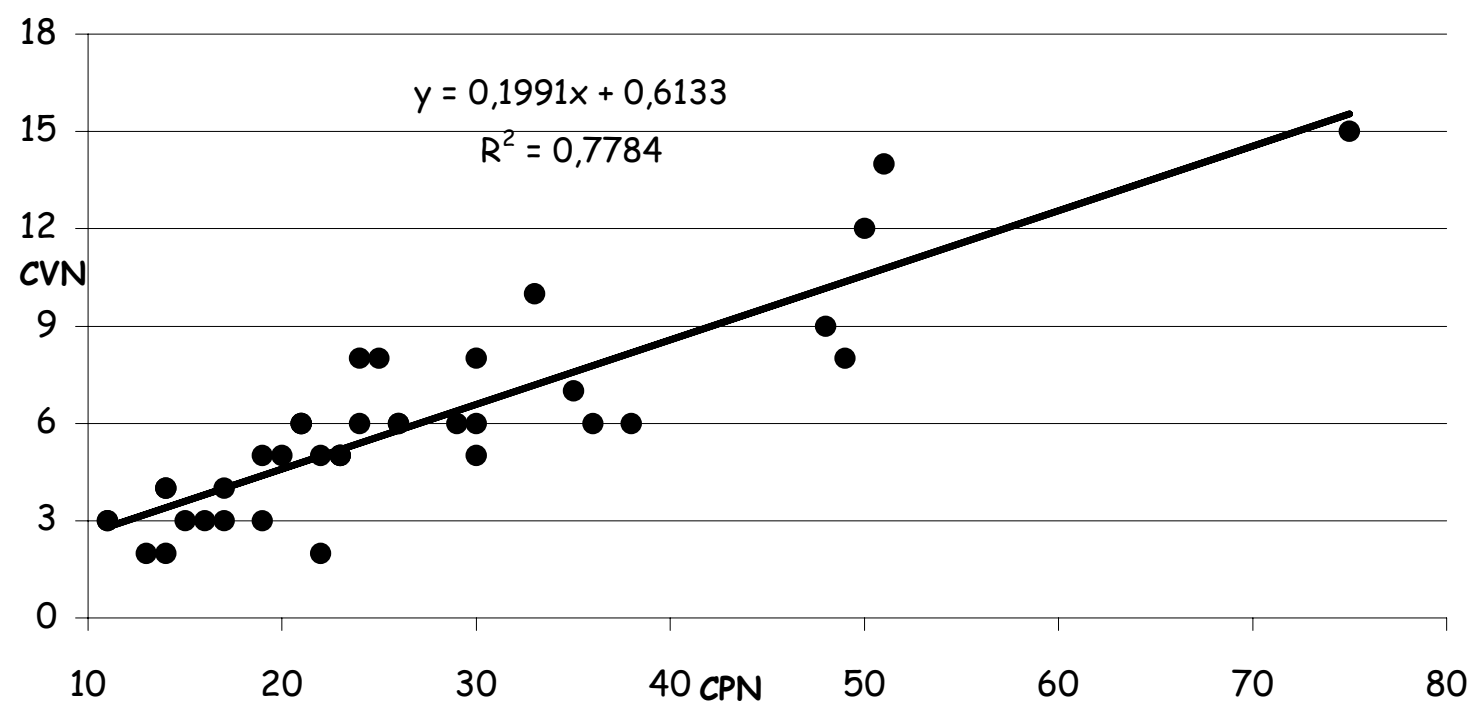

Figura VIII.15: Diagrama de dispersión y línea de regresión para CPN vs. CVN 


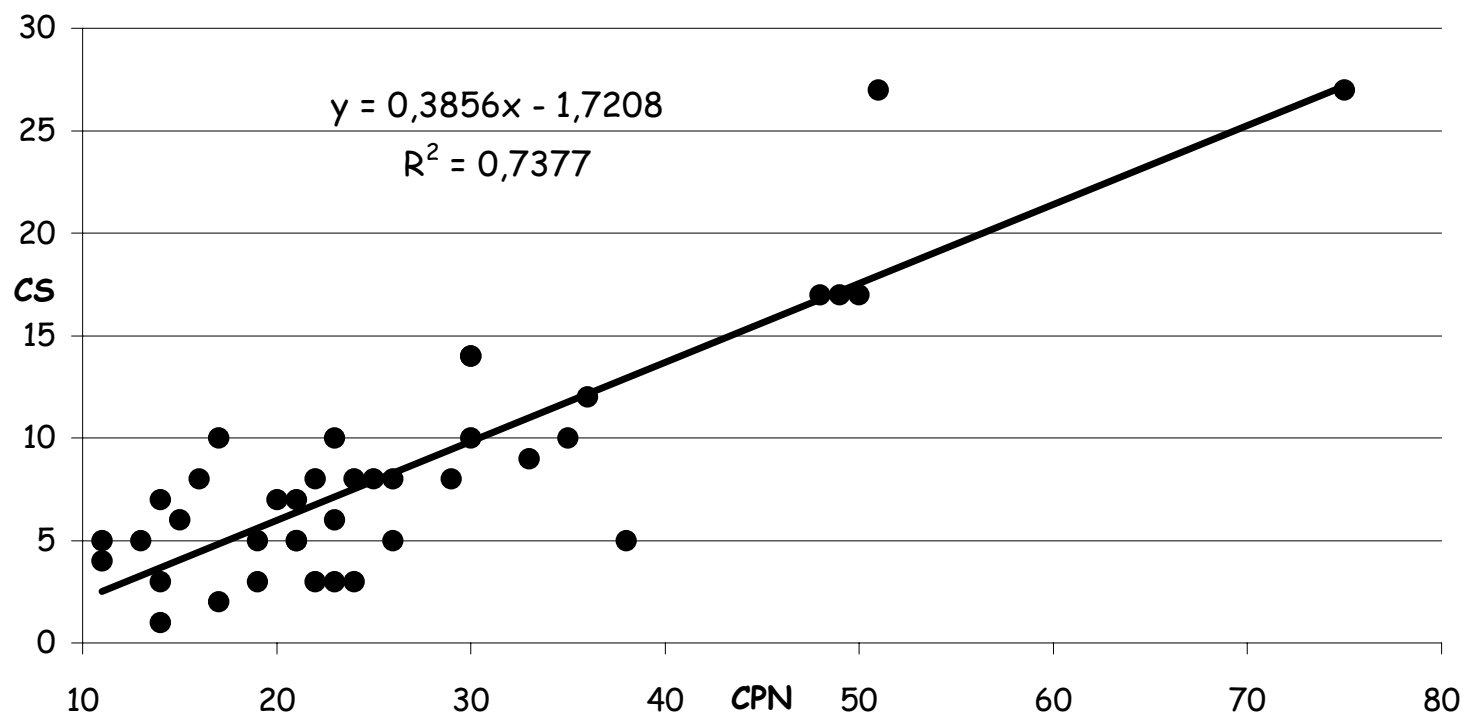

Figura VIII.16: Diagrama de dispersión y línea de regresión para CPN vs. CS

La cantidad de símbolos usados para definir una entrada presenta una mayor dispersión que los casos analizados hasta ahora, sobre todo para valores de CPN bajos. El 73,77\% de la variación de la cantidad de símbolos usados en las entradas del LEL (CS) está explicada por la línea de regresión, es decir por CPN.

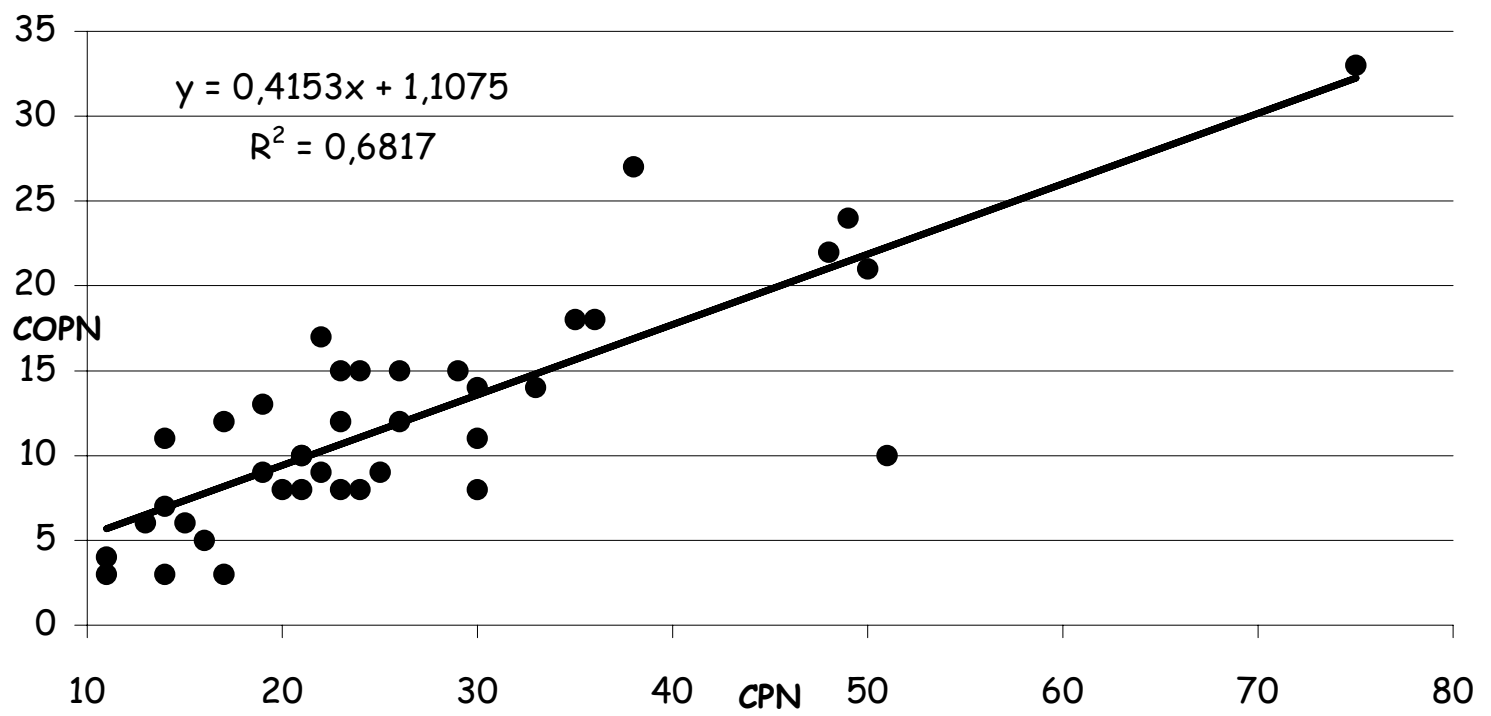

Figura VIII.17: Diagrama de dispersión y línea de regresión para CPN vs. COPN

Tal como se podía prever a partir de los análisis efectuados para la tendencia central y el comportamiento observado para cada entrada en particular en las Figuras VIII.2 y VIII.3 la mayor variación entre los valores registrados se da para la cantidad de otras palabras normalizadas. La Figura VIII.17 permite apreciar datos muy dispersos, alejados de la línea de regresión determinada. Sólo el 68,17\% de la variación en la cantidad de otras palabras normalizadas está explicado por CPN. 
En resumen, a partir de la observación de los resultados obtenidos en este análisis se concluye que la menor correlación de datos está dada entre la CPN y la COPN.

\section{RESUMEN DE LAS MEDICIONES}

TOTAL

MAXIMO

MINIMO

MEDIA

MEDIANA

MODA

DESV ST

CV

\section{R. CALCULADO (68\%) \\ R. ENCONTRADO $(80 \%)$}

\begin{tabular}{|c|c|c|c|c|c|c|c|c|c|}
\hline \multicolumn{10}{|c|}{ CSL $($ HOTEL $)=39$} \\
\hline$C P$ & $C P N$ & $C V N$ & CS & COPN & COER & COES & FO & FOI & TU \\
\hline 2616 & 1035 & 230 & 332 & 473 & - & - & - & - & - \\
\hline 194 & 75 & 15 & 27 & 33 & 0,57 & 1,67 & 72 & 10,33 & 1,85 \\
\hline 25 & 12 & 2 & 1 & 3 & 0,28 & 0,5 & 1 & 0,17 & 0,03 \\
\hline 67,08 & 26,54 & 5,90 & 8,51 & 12,13 & 0,40 & 1,00 & 8,50 & 1,37 & 0,24 \\
\hline 56 & 23 & 6 & 7 & 11 & & & & & \\
\hline \multirow[t]{5}{*}{31} & 23 & 6 & 5 & 8 & & & & & \\
\hline & 13,15 & 2,97 & 5,90 & 6,61 & & & & & \\
\hline & 0,5 & 0,49 & 0,65 & 0,55 & & & & & \\
\hline & $13 ; 40$ & $3 ; 9$ & $3 ; 14$ & $6 ; 19$ & & & & & \\
\hline & $10 ; 40$ & $3 ; 8$ & $3 ; 15$ & $5 ; 20$ & & & & & \\
\hline
\end{tabular}

Tabla VIII.15: Mediciones efectuadas al LEL "Hotel"

En la Tabla VIII.15 se consignan las mediciones más relevantes efectuadas al LEL "Hotel". Se hará un análisis de los datos pertenecientes al LEL completo, sin tener en cuenta las categorías establecidas.

Observando el ítem Total se nota que aproximadamente la mitad de las palabras usadas en este LEL son normalizadas y de ellas la mitad son palabras que no pertenecen a las clases verbo o símbolos del LEL. Por otro lado, de la mitad restante, un poco más de la mitad son símbolos y el resto verbos normalizados.

Los valores obtenidos para la mediciones de la tendencia central para cada clase de palabra, media, mediana y moda son muy cercanos entre sí, entonces se podría pensar que se trata de una distribución normal.

El coeficiente de variación, CV, toma valores mucho menores que la unidad, lo cual indica que los datos no están muy dispersos y por lo tanto, la media es una medición representativa de la población.

Los resultados más notables de esta tabla pertenecen a los rangos calculado y encontrado. El rango calculado se obtuvo a partir de considerar la distribución de los datos normal, aún cuando media, mediana y moda no son exactamente iguales. A partir de esta suposición, se calcula que el $68 \%$ de las mediciones deben estar entre los límites especificados. Por otro lado, al efectuar mediciones a cada símbolo, se pudo ver que aproximadamente el $80 \%$ de las mediciones están acotadas por lo rangos señalados como rango encontrado. Al comparar esto rangos se comprueba que coinciden en su gran mayoría, y cuando no lo hacen, sólo en difieren en \pm 1 palabra 
normalizada (excepto en el caso del límite inferior de la CPN que difiere en 3 palabras).

Después de este análisis se puede concluir diciendo que si se escribiera una nueva entrada para el LEL "Hotel", habría un $80 \%$ de probabilidad de que tuviera aproximadamente 26 palabras normalizadas entre las que 6 serían verbos y 9 serían símbolos del LEL.

El análisis de correlación indica que el mayor grado de dependencia entre las clases de palabras, se da para el par de datos CPN y CVN con un coeficiente de determinación de 0,7784

Los coeficientes calculados, COER, COES, FO, FOI y TU, sólo tiene sentido someterlos a un análisis en comparación con los mismos coeficientes calculados para otros LEL.

La validación definitiva de las medidas propuestas podrá surgir de un estudio detallado y comparativo de las mediciones aplicadas a diferentes LEL. 


\section{ANALISIS COMPARATIVO DE CASOS}

\section{ANÁLISIS DE LAS MEDICIONES OBTENIDAS}

Se compararán los valores obtenidos al aplicar el conjunto de mediciones definidas en los capítulos VI y VII a tres casos de estudio.

El primero es el LEL "Hotel" que se ha desarrollado como parte de este trabajo de tesis y que se puede consultar en el Apéndice E. El segundo es el LEL "Pasaporte" [Leite '96] en su versión original y el tercero es el LEL "LEL \& Escenarios" [García '00] que es un trabajo final de graduación.

Se buscaron ex profeso casos de estudio realizados por distintos grupos de personas y que tratan temas diferentes, esto asegura que el estilo de la redacción no es único y por lo tanto los datos con los que se trabajará en el proceso de medición serán suficientemente heterogéneos, esta cualidad de los datos es imprescindible para otorgarle mayor peso a las conclusiones.

Tanto el LEL "Hotel" como el LEL "Pasaporte" tienen aproximadamente la misma cantidad de entradas, no así el LEL "LEL \& Escenarios" que posee casi el doble.

\subsection{LEL "HOTEL"}

TOTAL

MAXIMO

MINIMO

MEDIA

MEDIANA

MODA

DESV ST

COEF. VARIACION

R. CALCULADO (68\%)

R. ENCONTRADO $(80 \%)$ COEF. DETERM. CPN

\begin{tabular}{||c|c|c|c|c|c|c|c|c|c||}
\hline \hline \multicolumn{10}{|c||}{ CSL(HOTEL) $=39$} \\
\hline CP & CPN & CVN & CS & COPN & COER & COES & FO & FOI & TU \\
\hline 2616 & 1035 & 230 & 332 & 473 & - & - & - & - & - \\
\hdashline 194 & 75 & 15 & 27 & 33 & 0,57 & 1,67 & 72 & 10,33 & 1,85 \\
\hdashline 25 & 12 & 2 & 1 & 3 & 0,28 & 0,5 & 1 & 0,17 & 0,03 \\
\hdashline 67,08 & 26,54 & 5,90 & 8,51 & 12,13 & 0,40 & 1,00 & 8,50 & 1,37 & 0,22 \\
\hdashline 56 & 23 & 6 & 7 & 11 & - & - & - & - & - \\
\hdashline 31 & 23 & 6 & 5 & 8 & - & - & - & - & - \\
\hdashline- & 13,15 & 2,97 & 5,90 & 6,61 & - & - & - & - & - \\
\hdashline- & 0,5 & 0,49 & 0,65 & 0,55 & - & - & - & - & - \\
\hdashline- & $13 ; 40$ & $3 ; 9$ & $3 ; 14$ & $6 ; 19$ & - & - & - & - & - \\
\hdashline- & $10 ; 40$ & $3 ; 8$ & $3 ; 15$ & $5 ; 20$ & 0,$3 ; 0,5$ & 0,$6 ; 1,2$ & $2 ; 10$ & 0,$2 ; 2$ & 0,$05 ; 0,3$ \\
\hdashline 0,9105 & - & 0,7784 & 0,7377 & 0,6817 & - & - & - & - & - \\
\hline
\end{tabular}

Tabla IX.1: Valores obtenidos de las mediciones aplicadas al LEL "Hotel"

Cabe destacar al observar los resultados de la Tabla IX.1 que para el LEL "Hotel" los valores obtenidos para la cantidad de palabras normalizadas, CPN, de la media, la mediana y la moda difiere entre ellos en un 15,39\% entre el mayor y el menor valor calculado, por lo tanto se puede considerar que las mediciones de la 
tendencia central prácticamente coinciden y en consecuencia responde a una distribución normal. Si se analiza la composición de la cantidad de palabras normalizadas, se destaca el hecho de que la cantidad de verbos normalizados, CVN, tiene una diferencia de sólo el $2 \%$ entre los valores obtenidos para las mediciones de la tendencia central. No sucede lo mismo con la cantidad de símbolos, CS y la cantidad de otras palabras normalizadas, COPN, aquí los porcentajes obtenidos son del $41 \%$ y $34 \%$ respectivamente. Los rangos calculados asumiendo que la distribución es normal (el $68 \%$ de las observaciones se encuentran a \pm 1 desvío estándar de la media) son muy próximos a los rangos encontrados por simple observación para aproximadamente el $80 \%$ de las mediciones. Entonces se puede asegurar que se está frente a una distribución normal de los datos.

Los coeficientes de variación están todos muy por debajo de la unidad, lo que indica una baja dispersión de los datos respecto de la media. Esto indica que la media es una medición representativa para esta población.

Entonces, se puede anticipar que el esfuerzo que demandará redactar una nueva entrada para este LEL será de aproximadamente 27 palabras normalizadas con una variación de \pm 13 palabras (valor correspondiente a un desvío estándar) de las cuales se puede esperar que $6 \pm 3$ sean verbos normalizados, $8 \pm 6$ sean otros símbolos del LEL y la cantidad de otras palabras normalizadas estará dada por $12 \pm 7$.

Obsérvese que ninguno de los límites superiores indicados superan lo valores máximos registrados para cada clase de palabra, tal como figura en la fila denominada MAXIMO de la Tabla IX.1

En cuanto a los coeficientes de determinación, $r^{2}$, que se calcularon en relación a la CPN, muestran una alta correlación de los datos para el caso de la CP. Los valores calculados para el resto de los pares de datos, también exhiben coeficientes altos, el menor de ellos corresponde a la COPN. Este resultado era de esperarse pues la mayor variación en los valores de las observaciones se encontró para esta medición.

\subsection{LEL "PASAPORTE"}

Los valores calculados para los parámetros de la tendencia central del LEL "Pasaporte" que figuran en la Tabla IX.2 indican a simple vista que la distribución de los datos no es normal. Específicamente, el porcentaje de diferencia entre el mayor y el menor valor registrado es de $61 \%$ para la CPN y para cada una de las clases de palabras los porcentajes son: $50 \%$ para la CVN, $60 \%$ para la CS y $27 \%$ para la COPN.

Teniendo en cuenta estos resultados y los valores obtenidos para los coeficientes de variación, los cuales son todos mayores de 0,5 e inclusive para la COPN supera la unidad, no se puede considerar esta distribución como normal. Por otro lado los valores de los desvío estándar son elevados para la CPN y la COPN, lo que indica que las observaciones están muy dispersas respecto de la media de la población.

Entonces los rangos calculados se obtuvieron teniendo en cuenta el Teorema de Chebyshev que dice que independientemente de cómo sea una distribución, se espera que el $75 \%$ de las observaciones estén entre \pm 2 desvíos estándar. Al comparar estos valores con los registrados para el $80 \%$ de las mediciones, se encuentra que los rangos son muy similares. Aún cuando este conjunto de datos 
cumple con el Teorema de Chebyshev, el autor no considera que pueda hacer predicciones sobre el esfuerzo de redactar una nueva entrada de este LEL.

TOTAL

MAXIMO

MINIMO

MEDIA

MEDIANA

MODA

DESV ST

COEF. VARIACION

R. CALCULADO (75\%)

R. ENCONTRADO (80\%) COEF. DETERM. CPN

\begin{tabular}{||c|c|c|c|c|c|c|c|c|c||}
\hline \hline \multicolumn{10}{|c||}{ CSL(PASAPORTE) $=37$} \\
\hline CP & CPN & CVN & CS & COPN & COER & COES & FO & FOI & TU \\
\hline 2251 & 1057 & 220 & 281 & 556 & - & - & - & - & - \\
\hdashline 240 & 117 & 17 & 25 & 90 & 0,68 & 2 & 67 & 5,36 & 1,81 \\
\hdashline 21 & 8 & 1 & 1 & 0 & 0,32 & 0,5 & 0 & 0 & 0 \\
\hdashline 60,84 & 28,57 & 5,95 & 7,59 & 15 & 0,46 & 1,04 & 7,57 & 1,01 & 0,2 \\
\hdashline 49 & 20 & 4 & 7 & 11 & - & - & - & - & - \\
\hdashline 30 & 11 & 3 & 3 & 12 & - & - & - & - & - \\
\hdashline- & 21,47 & 4,11 & 4,99 & 15,49 & - & - & - & - & - \\
\hdashline- & 0,75 & 0,69 & 0,66 & 1,03 & - & - & - & - & - \\
\hdashline- & $0 ; 70$ & $0 ; 14$ & $0 ; 18$ & $0 ; 46$ & - & - & - & - & - \\
\hdashline- & $10 ; 40$ & $2 ; 10$ & $2 ; 11$ & $5 ; 30$ & 0,$35 ; 0,5$ & 0,$6 ; 1,3$ & $1 ; 14$ & 0,$1 ; 3$ & 0,$03 ; 0,4$ \\
\hdashline 0,9668 & - & 0,7149 & 0,4637 & 0,8877 & - & - & - & - & - \\
\hline \hline
\end{tabular}

Tabla IX.2: Valores obtenidos de las mediciones aplicadas al LEL "Pasaporte"

Los coeficientes de determinación indican, una alta correlación entre la cantidad de palabras y la cantidad de palabras normalizadas y una muy baja correlación para la cantidad de símbolos.

\subsection{LEL "LEL \& EscENARIOS"}

TOTAL

MAXIMO

MINIMO

MEDIA

MEDIANA

MODA

DESV ST

COEF. VARIACION

R. CALCULADO (68\%)

R. ENCONTRADO (80\%) COEF. DETERM. CPN

\begin{tabular}{||c|c|c|c|c|c|c|c|c|c||}
\hline \multicolumn{10}{|c||}{ CSL(LEL \& ESCENARIOS) = 78 } \\
\hline CP & CPN & CVN & CS & COPN & COER & COES & FO & FOI & TU \\
\hline 4869 & 2096 & 441 & 662 & 993 & - & - & - & - & - \\
\hdashline 275 & 121 & 22 & 59 & 42 & 0,73 & 2,25 & 111 & 7,33 & 1,42 \\
\hdashline 21 & 9 & 0 & 3 & 3 & 0,28 & 0 & 1 & 0,11 & 0,01 \\
\hdashline 62,42 & 26,87 & 5,65 & 8,49 & 12,73 & 0,44 & 1,05 & 8,46 & 1,09 & 0,11 \\
\hdashline 51 & 23,5 & 5 & 7 & 12 & - & - & - & - & - \\
\hdashline 42 & 27 & 4 & 7 & 5 & - & - & - & - & - \\
\hdashline- & 16,44 & 3,61 & 6,89 & 7,7 & - & - & - & - & - \\
\hdashline- & 0,61 & 0,64 & 0,81 & 0,60 & - & - & - & - & - \\
\hdashline- & $10 ; 43$ & $2 ; 9$ & $2 ; 15$ & $5 ; 20$ & - & - & - & - & - \\
\hdashline- & $13 ; 40$ & $3 ; 9$ & $4 ; 12$ & $5 ; 18$ & 0,$35 ; 0,5$ & 0,$5 ; 1,25$ & $2 ; 15$ & 0,$1 ; 1,5$ & 0,$01 ; 0,14$ \\
\hdashline 0,9291 & - & 0,7002 & 0,757 & 0,7229 & - & - & - & - & - \\
\hline \hline
\end{tabular}

Tabla IX.3: Valores obtenidos de las mediciones aplicadas al LEL "LEL \& Escenarios" 
Las mediciones obtenidos para el LEL "LEL \& Escenarios" figuran en la Tabla IX.3. La comparación de los valores calculados para la media, la mediana y la moda indican que el porcentaje de la diferencia entre los valores extremos es ínfimo para la cantidad de palabras normalizadas, $0,48 \%$. Para la cantidad de verbos y para la cantidad de símbolos es del $29 \%$ y $18 \%$ respectivamente y la mayor diferencia corresponde a la COPN con el $61 \%$. Los coeficientes de variación son valores bajos, excepto en el caso de la cantidad de símbolos que llega a 0,81. Aún teniendo en cuenta este valor, se considera que la distribución es normal, pues el porcentaje de la diferencia entre las mediciones de la tendencia central para la población, es decir la cantidad de palabras normalizadas, es cercano a cero.

Si la distribución es normal, entonces los rangos calculados se pueden obtener teniendo en cuenta \pm 1 desvío estándar a partir de la media. Se pueden comparar estos valores con los correspondientes a los rangos encontrados por simple observación de las lecturas registradas para el $80 \%$ de las mediciones en la Tabla 1 del Apéndice H. Los rangos encontrados son mucho más estrechos que los previstos.

Entonces se puede concluir que el esfuerzo de escribir una nueva entrada para este LEL demandará aproximadamente $27 \pm 16$ palabras normalizadas, de las cuales 6 \pm 4 serán verbos normalizados, $8 \pm 7$ serán símbolos del LEL y $13 \pm 8$ serán otras palabras normalizadas. Nuevamente, ninguno de los máximos previstos supera las mediciones consignadas en la fila rotulada MAXIMO de la Tabla IX.3.

En la fila rotulada MINIMO la Tabla IX.3 se registra un valor 0 para la CVN y para el COES esto significa que al menos una definición de este LEL no contiene verbos normalizados.

Los coeficientes de determinación, como en los casos anteriores, presentan una alta dependencia entre la cantidad de palabras y la cantidad de palabras normalizadas. El resto de los coeficientes tienen valores muy similares que indican que aproximadamente el $70 \%$ de las variaciones en la cantidad de otras palabras normalizadas, la cantidad de verbos normalizados y la cantidad de símbolos, puede explicarse por la variación de la cantidad de palabras normalizadas.

\section{Comparación de los tres Casos de Estudio}

Los casos de estudio tienen una cantidad de palabras similar entre "Hotel" y "Pasaporte" y muy diferente a "LEL \& Escenarios", tal como puede apreciarse en la Tabla IX.4.

\begin{tabular}{|l|c|c|c|}
\cline { 2 - 4 } \multicolumn{1}{c|}{} & CP & CPN & $\%$ \\
\hline “Hotel" & 2616 & 1035 & 40 \\
\hline "Pasaporte" & 2251 & 1057 & 47 \\
\hline “LEL \& Escenarios" & 4869 & 2096 & 43 \\
\hline
\end{tabular}

Tabla IX.4: Totales para la cantidad de palabras y cantidad de palabras normalizadas

El porcentaje de la cantidad de palabras normalizadas respecto a la cantidad de palabras usadas en las definiciones es muy similar. Para los tres casos, en promedio se obtiene que el $43,33 \%$ del total de las palabras usadas son normalizadas. 
Los tres LEL exhiben una distribución porcentual similar para las clases de palabras normalizadas, según se observa en la Tabla IX.5. Aproximadamente el $50 \%$ corresponde a otras palabras, el $30 \%$ a símbolos del propio LEL y el $20 \%$ a verbos.

\begin{tabular}{|l|c|c|c||}
\cline { 2 - 4 } \multicolumn{1}{l|}{ TOTAL\% } & CVN & CS & COPN \\
\hline “Hotel” & 22 & 32 & 45 \\
\hline “Pasaporte" & 21 & 27 & 52 \\
\hline “LEL \& Escenarios" & 21 & 31 & 48 \\
\hline
\end{tabular}

Tabla IX.5: Distribución porcentual de las clases de palabras

\begin{tabular}{|c|c|c|c|}
\hline & \\
\hline & & $C P$ & CPN \\
\hline \multirow{3}{*}{$\begin{array}{l}\bar{\Phi} \\
\text { 후 }\end{array}$} & DESV ST & - & 13,15 \\
\hline & COEF. VARIACION & - & 0,5 \\
\hline & COEF. DETERM. CPN & $0,9105:$ & \\
\hline \multirow{3}{*}{ 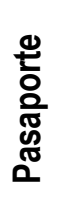 } & DESV ST & - & 21,47 \\
\hline & COEF. VARIACION & 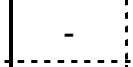 & 0,75 \\
\hline & COEF. DETERM. CPN & 0,9668 & - \\
\hline \multirow{3}{*}{ 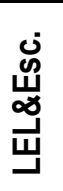 } & DESV ST & & 16,44 \\
\hline & COEF. VARIACION & & 0,61 \\
\hline & COEF. DETERM. CPN & 0,9291 & - \\
\hline
\end{tabular}

Tabla IX.6: Valores de la dispersión para la cantidad de palabras normalizadas

El desvío estándar evalúa el error que se comete al usar la media de la población como característica de cualquier individuo, por lo tanto se espera que el valor de este parámetro sea bajo con respecto a la media. El desvío estándar es sensible a la cantidad y magnitud de las mediciones extremas registradas para la población. Según la Tabla IX.6 los LEL "Hotel" y "LEL \& Escenarios" registran los resultados más bajos y similares entre sí.

El coeficiente de variación es una medida que evidencia mejor la distribución de los datos pues relaciona el desvío estándar con la media, permitiendo la comparación del grado de dispersión entre dos o mas poblaciones. Cuanto menor que la unidad sea el coeficiente, más homogéneo será el conjunto de datos. Al comparar los valores de la dispersión para los LEL en estudio, se concluye que el "Hotel" y el "LEL \& Escenarios" tienen valores del coeficiente similares, no así el "Pasaporte". El coeficiente de variación también debería considerarse como una medida de la calidad de un LEL.

Se comparan los valores del coeficiente de determinación, $r^{2}$ para los tres casos de estudio. Este coeficiente pone de manifiesto el grado de asociación entre variables, en este caso la cantidad de palabras respecto a la cantidad de palabras normalizadas de una entrada dada. Si el coeficiente fuera igual a la unidad, entonces 
la dependencia entre los grupos de datos sería directa. En los casos presentados, el valor del coeficiente es muy cercano a 1 , entonces se puede concluir diciendo que es muy alta la asociación entre la cantidad de palabras y la cantidad de palabras normalizadas para los tres casos de estudio.

\begin{tabular}{|l|c:c:c|}
\cline { 2 - 4 } \multicolumn{1}{l|}{$\mathrm{R}^{2}$} & CVN & CS & COPN \\
\hline \hline HOTEL & 0,7784 & 0,7377 & 0,6817 \\
\hline PASAPORTE & 0,7149 & 0,4637 & 0,8877 \\
\hline LEL\&ESCENARIOS & 0,7002 & 0,757 & 0,7229 \\
\hline
\end{tabular}

Tabla IX.7: Valores para los coeficientes de determinación para todas las clases de palabras con respecto a CPN

No se observan tendencias para las otras clases de palabras como puede deducirse a partir de la Tabla IX.7

En el análisis que sigue se ha determinado para las mediciones CPN, FO, FOI, TU, COER y COES de los tres LEL el porcentaje de diferencia entre el mayor y el menor valor registrado para las mediciones extremas y para la media con el objeto de identificar posibles tendencias para cualquier LEL. Se ha visto que estos porcentajes oscilan en un 30\% para las mediciones extremas, este valor se considera lo suficientemente alto como para que no se pueda hacer predicciones sobre los valores extremos, ya sean máximos o mínimos. En general no ocurre lo mismo con la media, los porcentajes registrados son relativamente bajos, cercanos al $10 \%$ con algunas excepciones que se plantearán para cada medición. Si además se tiene en cuenta que se pudo establecer una distribución normal para las mediciones del LEL y que los valores registrados par el coeficiente de variación son bajos (ver Tabla IX.6) entonces se puede concluir que la media calculada para cada medición es representativa de cualquier entrada del LEL.

Por otro lado existen notables coincidencias entre los tres casos de estudio para los rangos observados para el $80 \%$ de los valores de cada una de las mediciones. Este dato permitiría establecer valores probables con bastante certeza para una medición dada de cualquier LEL.

Para CPN, según los valores calculados en la Tabla IX.8 (b), la media tiene una amplitud del $6 \%$ entre mediciones extremas, una cantidad realmente ínfima. La media de las medias es 27,33. Este valor coincide muy aproximadamente con los valores anticipados para esta medición en los parágrafos 1.1 y 1.3 .

En los tres casos el extremo superior del rango hallado para el $80 \%$ de las observaciones es de 40 palabras y el mínimo está entre 10 y 13 . Este valor es muy cercano al mínimo de 9,67 obtenido como media de los valores mínimos. 


\begin{tabular}{|c|c|c|}
\hline & & CPN \\
\hline \multirow{4}{*}{$\begin{array}{l}\bar{\Phi} \\
\text { 오 }\end{array}$} & MAXIMO & 75 \\
\hline & MINIMO & 12 \\
\hline & MEDIA & 26,54 \\
\hline & RANGO (80\%) & $10 ; 40$ \\
\hline \multirow{4}{*}{ 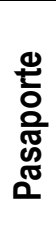 } & MÁXIMO & 117 \\
\hline & MINIMO & 8 \\
\hline & MEDIA & 28,57 \\
\hline & RANGO $(80 \%)$ & $10 ; 40$ \\
\hline \multirow{4}{*}{$\begin{array}{l}\text { Ш } \\
\text { Ш } \\
\end{array}$} & MÁXIMO & 121 \\
\hline & MINIMO & 9 \\
\hline & MEDIA & 26,87 \\
\hline & RANGO (80\%) & $13 ; 40$ \\
\hline
\end{tabular}

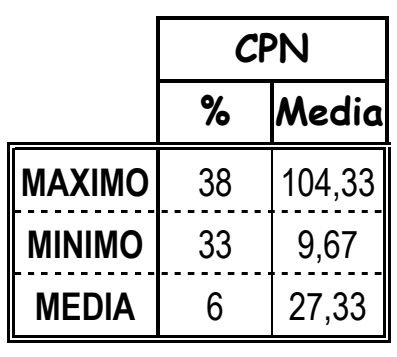

(b)

(a)

Tabla IX.8: Valores extremos, medios y encontrados para el $80 \%$ de las observaciones de la cantidad de palabras normalizadas

\begin{tabular}{|c|c|c|c|c|}
\hline & & & \\
\hline & & FO & FOI & TU \\
\hline \multirow{4}{*}{$\begin{array}{l}\bar{\Phi} \\
\text { 오 }\end{array}$} & MAXIMO & 72 & 10,33 & 1,85 \\
\hline & MINIMO & 1 & 0,17 & 0,03 \\
\hline & MEDIA & 8,50 & 1,37 & 0,22 \\
\hline & RANGO (80\%) & $2 ; 10$ & 0,$2 ; 2$ & 0,$05 ; 0,30$ \\
\hline \multirow{4}{*}{ 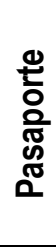 } & MÁXIMO & 67 & 5,36 & 1,81 \\
\hline & MINIMO & 0 & 0 & 0 \\
\hline & MEDIA & 7,57 & 1,01 & 0,20 \\
\hline & RANGO $(80 \%)$ & $1 ; 14$ & 0,$1 ; 3$ & 0,$03 ; 0,40$ \\
\hline \multirow{4}{*}{$\begin{array}{l}\boldsymbol{\infty} \\
\underset{\uplus}{山}\end{array}$} & MÁXIMO & 111 & 7,33 & 1,42 \\
\hline & MINIMO & 1 & 0,11 & 0,01 \\
\hline & MEDIA & 8,46 & 1,09 & 0,11 \\
\hline & RANGO (80\%) & $2 ; 15$ & 0,$1 ; 1,5$ & 0,$01 ; 0,14$ \\
\hline
\end{tabular}

\begin{tabular}{||c|c|c|c|c|c|c|}
\cline { 2 - 7 } \multicolumn{1}{c|}{} & \multicolumn{2}{c|}{ FO } & \multicolumn{2}{c|}{ FOI } & \multicolumn{2}{c|}{ TU } \\
\cline { 2 - 7 } \multicolumn{1}{c|}{} & $\%$ & Media & $\%$ & Media & $\%$ & Media \\
\hline \hline MAXIMO & 35,14 & 83 & 48 & 7,67 & 23,24 & 1,69 \\
\hdashline MINIMO & - & - & - & - & - & - \\
\hdashline MEDIA & 10,94 & 8,18 & 26,28 & 1,16 & 50 & 0,18 \\
\hline
\end{tabular}

(a)

Tabla IX.9: Valores extremos, medios y rango del $80 \%$ de las observaciones para los coeficientes FO, FOI y Tasa de uso

El Fan Out habla del grado de utilización de un símbolo para definir otros símbolos, está relacionado con el principio de circularidad que recomienda el uso del vocabulario del LEL para elaborar nuevas definiciones. Por lo tanto se lo podría 
considerar una medición de calidad del LEL, entonces se podría concluir diciendo que aquél LEL que tenga los valores de FO más altos, será el de mejor calidad.

Para el FO en los casos de estudio que se abordaron en esta tesis, se encuentra que los valores de la media se diferencian en un 10,94\% entre el valor mayor y el menor, por lo tanto se puede considerar el promedio de la media como una buena aproximación siendo este valor 8,18.

En cuanto al rango encontrado para el $80 \%$ de los datos, se podría decir que responden a una estructura similar.

Los coeficientes FOI y TU se calculan teniendo como numerador la medición FO, en los casos en que éste es 0 los coeficientes serán 0 para esas entradas.

EI FOI está directamente relacionado al FO pues es el cociente entre el FO y el FI, evalúa la importancia de un símbolo en función de las veces que es usado para definir otros símbolos y la cantidad de símbolos que usa en su propia definición. Un FOI alto corresponde a símbolos importantes que definen conceptos fundamentales para el contexto pues son muy usados para definir otros símbolos. El porcentaje de la diferencia entre valores extremos de la media, según la Tabla IX.9 (a) es 26,28\%, valor suficientemente alto como para no considerar la media como representativa del FOI. Los rangos encontrados para aproximadamente el $80 \%$ de las entradas de cada uno de los tres LEL no permiten anticipar valores para un LEL cualquiera.

EI TU relaciona el FO de cada entrada con el total de entradas definidas, también es una medida del uso de una entrada respecto a todas las entradas definidas para un LEL dado. Para los valores medios existe entre las mediciones extremas un $50 \%$ de diferencia por ello no se tendrán en cuenta para caracterizar tendencias.

Merece un análisis especial los valores registrados para el TU del LEL "LEL \& Escenarios", si bien este LEL tiene el mayor valor máximo de FO, 111, esto se compensa por la cantidad más alta de entradas, 78, dando así los valores menores de la Tabla IX.9 (a) para ese coeficiente.

El coeficiente de redundancia, COER, relaciona la cantidad de palabras normalizadas con la cantidad de palabras usadas en una definición dada del LEL.

El porcentaje calculado para la diferencia entre los valores extremos de la media que se consigna en la Tabla IX.10 (b) es relativamente bajo, llega a 13,04\%

Los rangos en los que se encontraron el $80 \%$ de los valores, como se puede apreciar en la Tabla IX.10 (a) son prácticamente idénticos, lo que refuerza la validez de los valores medios calculados.

El coeficiente de simplicidad, COES, relaciona la cantidad de verbos normalizados y la cantidad de oraciones que tiene una entrada del LEL.

Según puede apreciarse en la Tabla IX.10 (b) el porcentaje para la diferencia entre las mediciones extremas para la media es de 4,76\%, valor este muy bajo. 


\begin{tabular}{|c|c|c|c|}
\hline & \\
\hline & & COER & COES \\
\hline \multirow{4}{*}{$\begin{array}{l}\bar{\Phi} \\
\text { 우 }\end{array}$} & MAXIMO & 0,57 & 1,67 \\
\hline & MINIMO & 0,28 & 0,5 \\
\hline & MEDIA & 0,40 & 1,00 \\
\hline & RANGO (80\%) & 0,$3 ; 0,5$ & 0,$6 ; 1,20$ \\
\hline \multirow{4}{*}{ 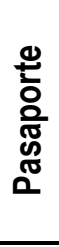 } & MÁXIMO & 0,68 & 2 \\
\hline & MINIMO & 0,32 & 0,5 \\
\hline & MEDIA & 0,46 & 1,04 \\
\hline & RANGO $(80 \%)$ & 0,$35 ; 0,5$ & 0,$6 ; 1,30$ \\
\hline \multirow{4}{*}{$\begin{array}{l}\text { Ш } \\
\text { Ш }\end{array}$} & MÁXIMO & 0,73 & 2,25 \\
\hline & MINIMO & 0,28 & 0 \\
\hline & MEDIA & 0,44 & 1,05 \\
\hline & RANGO (80\%) & 0,$35 ; 0,5$ & 0,$5 ; 1,25$ \\
\hline
\end{tabular}

\begin{tabular}{|c|c|c|c|c|}
\cline { 2 - 5 } \multicolumn{1}{c|}{} & \multicolumn{2}{c|}{ COER } & \multicolumn{2}{c|}{ COES } \\
\cline { 2 - 5 } \multicolumn{1}{c|}{} & $\%$ & Media & $\%$ & Media \\
\hline MAXIMO & 21,92 & 0,66 & 25,78 & 1,97 \\
\hdashline MINIMO & 12,5 & 0,29 & 100 & 0,33 \\
\hdashline MEDIA & 13,04 & 0,43 & 4,76 & 1,03 \\
\hline
\end{tabular}

(a)

Tabla IX.10: Valores extremos, medios y rango del $80 \%$ de las observaciones de los coeficientes de redundancia y simplicidad

\section{Valores Esperados para las Mediciones}

Como síntesis del análisis realizado se pueden establecer algunos valores esperados para las mediciones aplicadas al LEL. Por supuesto un mayor grado de experimentación ayudará a realizar mejores predicciones.

Del total de palabras utilizadas para construir las entradas de un LEL dado, el $43,33 \%$ serán palabras normalizadas y de éstas el $20 \%$ serán verbos, el $30 \%$ símbolos y el $50 \%$ restante otras palabras.

El $80 \%$ de los símbolos tendrá entre 40 y 10 palabras normalizadas. El término medio de palabras normalizadas será 27 con una amplitud del $6 \%$.

Se espera una alta correlación entre la cantidad de palabras y la cantidad de palabras normalizadas, no ocurre lo mismo con los otros tipos de palabras respecto a la cantidad de palabras normalizadas.

Se ha visto que el LEL responde bastante bien a una distribución normal, esto indica que el valor de la media será, para la mayoría de las mediciones, representativo para cualquiera de los elementos del conjunto.

El valor esperado para el FO de una entrada es de 8 . El rango para el $80 \%$ de las observaciones oscila entre 1 y 15.

Tanto para el FOI como para el TU no se encontraron tendencias para ninguno de los parámetros evaluados.

En cuanto al COER el valor esperado para la media es de 0,43 y el $80 \%$ de los valores de esta medición estarán entre 0,3 y 0,5.

EI COES presenta una media de 1,03 con un rango esperado para el $80 \%$ de las observaciones entre 0,5 y 1,30 . 


\section{CAPITULOX}

\section{CONCLUSIONES}

\section{CONCLUS IONES}

En este trabajo se ha demostrado que es posible establecer mediciones para un texto en Lenguaje Natural que permita estimar su tamaño y en algunos casos también su calidad.

$\mathcal{A}$ partir de establecer el concepto de Palabra Normalizada, que es aquélla imprescindible en una definición para transmitir un concepto, se establecie ron Reglas de Normalización ${ }^{1}$ que permiten determinar, sin ninguna duda, si una palabra tiene la cualidad de ser normalizada.

Tomando en cuenta sólo las palabras normalizadas, se ha podido crear un conjunto de definiciones donde se establecen con rigurosidad matemática las características de las mediciones concebidas para evaluar el tamaño del Léxico Extendido del Lenguaje o LEL y de cada una de sus entradas. Estas mediciones aplicadas al LEL "Hotel" en forma exhaustiva permitieron darles la forma definitiva. Posteriormente se aplicaron a dos casos de estudio más para establecer comparaciones entre ellos.

Estas mediciones son para cada simbolo del LEL: cantidad de palabras, cantidad de palabras normalizadas, cantidad de verbos normalizados, cantidad de simbolos (que intervienen en la definición), cantidad de otras palabras normalizadas (que no son ni verbos ni simbolos), cantidad de oraciones y los coeficientes de redundancia y simplicidad; para el simbolo en relación al conjunto: Fan Out (cantidad de veces que un simbolo es usado para definir otros simbolos), FO I (relación entre la cantidad de veces que un simbolo es usado para definir otros simbolos y la cantidad de simbolos que utiliza en su propia definición), tasa de uso (relación entre la cantidad de veces que un simbolo es usado para definir otros simbolos y la cantidad de simbolos de ( LEL); para el LEL: cantidad de simbolos, cantidad de simbolos en cada grupo según la clasificación general dada para las entradas [Hadad 97], [Leite '00], cantidad de palabras, cantidad de verbos normalizados, cantidad de simbolos, cantidad de otras palabras normalizadas y las mediciones estadisticas: media, mediana, moda, desvio estándar, coeficiente de variación y coeficientes de de terminación.

El autor ha podido comprobar que al pretender establecer mediciones de tamaño, se ha encontrado que algunas de ellas son, en realidad de calidad. Por ejemplo las cantidades de oraciones, palabras normalizadas, verbos, otras palabras normalizadas son netamente mediciones de tamaño. Pero hay otras como la cantidad de simbolos o Fan In, el Fan Out, el FOI, el $\mathcal{T} \mathcal{U}$, los coeficientes de redundancia y

\footnotetext{
${ }^{1}$ Ver Apéndice $\mathcal{B}$

${ }^{2}$ Ver Apéndice $C$
} 
simplicidad que además de estar representadas por un número, la magnitud de ese núme ro evidencia característic as que cualifican el LEL.

La cantidad de palabras normalizadas es aproximadamente el $43 \%$ del total de palabras usadas en las definiciones (este porcentaje es el mismo ya sea que se trate del LEL como de cada entrada).

Se ha visto que las clases de palabras se reparten en todos los casos en los mismos porcentajes. Aproximadamente el $50 \%$ corresponde a otras palabras normalizadas, el $30 \%$ a simbolos del propio LEL y el $20 \%$ a verbos normalizados.

Después de analizar los tres casos de estudio se ha llegado a la conclusión de que se podría esperar que un simbolo de un LEL tenga aproximadamente 104 palabras normalizadas como máximo con una amplitud del $38 \%$, 10 palabras normalizadas como minimo con una amplitud del $33 \%$ y como término medio 27 palabras normalizadas con una amplitud del $6 \%$.

El análisis de correlación indic a valores llamativamente altos delcoeficiente de de terminación cuando se comparan la cantidad de palabras con la cantidad de palabras normalizadas en los tres casos de estudio. Esto sugiere una muy alta la asociación entre estas cantidades. No ocurre lo mismo con las otras clases de palabras.

Un comportamiento especialmente llamativo se manifiesta en los rangos observados para el $80 \%$ de los valores que resultan muy similares para cada me dición en los tres casos de estudio. Sin embargo, para poder afirmar los valores de los rangos, habría que medir otros LEL.

$\mathcal{A l}$ realizar el estudio en profundidad del LEL "Hotel" se concluyó que la inclusión de los valores extremos, aquéllos que quedan fuera de los límites calculados si la distribución fuese normal, no afecta los valores de las mediciones de la tendencia central. Generalizando este concepto para los otros LEL, se analizaron las medidas de la tendencia central para saber si la distribución de los datos era normal. Se compararon la media, la mediana y la moda para las palabras normalizadas y para cada clase de palabra. En los casos de estudio "Hotel" y "LEL ef Escenarios" se concluyó que la distribución es normal, tanto para la cantidad de palabras normalizadas como para las clases de palabras, no ocurre lo mismo con el caso de estudio "Pasaporte" donde la única distribución que podría considerarse normal corresponde a la cantidad de otras palabras normalizadas. Por lo tanto la forma de la distribución de los datos en el LEL es un te ma que queda supeditado a una mayor investigación.

$S i$ bien se han observado amplias fluctuaciones en la cantidad de otras palabras normalizadas cuando se analizó cada entrada por se parado, cuando se e valúa el LEL, esta variaciones se equiparan y se obtienen valores similares alcomparar los tres casos de estudio.

\section{PAUTAS PARA LA CONSTRUCCIÓN $\mathcal{N}$ TE UN LEL MEDI BLE}

Después de efectuar mediciones a tres casos de estudio, se ha observado la conveniencia de establecer ciertas recomendaciones para generar un buen $L E \mathcal{L}$ (conciso, claro, estructurado) que por otro lado sea factible de ser medido.

Ellas son:

- Se preferirán oraciones simples, con un solo suje to y verbo.

- Siempre que sea posible, se preferirá usar símbolos del LEL a otras palabras 
- Cuando la misma acción se efectúe sobre distintos sujetos, éstos se separarán mediante comas.

- Cuando distintas acciones se efectúen sobre el mismo sujeto, éstas se separarán mediante comas, generándose así una oración con varios verbos.

- Es deseable para obtener una buena definición de un simbolo del Lelque de todas las palabras utilizadas, el $50 \%$ sean palabras normalizadas.

- Es deseable que la cantidad de palabras normalizadas (CPN) contenga un $50 \%$ de otras palabras normalizadas (COPN(L) y el restante $50 \%$ esté distribuido en partes similares entre verbos normalizados (CVN) y simbolos del LEL (CS).

- En la matriz de adyacencia ponderada la diagonal principal debería contener únicamente o; si así no sucede, significa que para definir una entrada se usó la misma entrada, algo que no es aconsejable para ninguna definición.

- Si en la definición de una entrada del LEL se utiliza la palabra que se está definiendo como parte del texto, ésta se debe reemplazar por un sinónimo o bien se cuenta como una palabra cualquiera, pero nunca como un simbolo del LEL que interviene en la Cantidad de Simbolos (CS) o Fan In.

- Si se definen entradas que finalmente se decide no relevarlas, no deberían ser conside radas simbolos del LEL, aún cuando sean conce ptos usados en el Udd pues el aporte de información que realizan a la fiora de establecer medidas no es completo.

- Si existen varias entradas no relevadas no se puede asegurar que los resultados de las mediciones sean útiles para evaluar el LEL en cuestión.

- Cuando después de realizar las mediciones se encuentran entradas que tienen un Fan Out nulo, de berían reconsiderarse dos hechos:

1) Ca entrada no se usa en el dominio y por lo tanto no resulta de importancia en la modelización, entonces podría eliminarse

2) se usa el concepto que define la entrada pero con otras palabras, entonces habría que redactar nuevamente las entradas que hacen alusión al concepto expresado por esas entradas de $\mathcal{F O}=0$

\section{TRABAg OS FUTUROS}

El autor considera que el tema de las mediciones no se agota en este trabajo. Como próximos trabajos se propone:

Incrementar la experimentación

Pese a los casos de estudio realizados es necesario aplicar a nuevos casos de estudio las mediciones propuestas para contar con un conjunto de datos más amplio que permita refinarlas y establecer relaciones más precisas entre ellas. En particular se tratará de utilizar las técnicas del área de la estadística de que permiten mejorar la caracterización de las entradas teniendo en cuenta en forma simultánea el conjunto de todas las variables de interés (Análisis multivariado).

Ecuación de tamaño

Investigar la existencia de ecuaciones que relacionen las medidas propuestas para obtener una medición únic a para cada entrada del LEL y para cada LEL. 
Herramienta automática

Estudiar la posibilidad de construir una herramienta que soporte realizar las mediciones as istiendo al ingeniero de requerimientos y generando una base de datos con información de casos.

Especificación del proceso

Especificar el proceso de mediciones en términos que lo conviertan en una herramienta de utilización práctica en proyectos de construcción de software.

Estimación de esfuerzo

Establecer una estimación delesfuerzo necesario para hacer las mediciones de tamaño y estimaciones de esfuerzo de construcción de los escenarios a partir del tamaño del $\mathcal{L E L}$.

Mediciones para los Escenarios

Extender el enfoque propuesto para utilizarlo en el establecimiento de mediciones de tamaño para los Escenarios del enfoque de Leite. Sobre esa base se podrian identificar relaciones entre el tamaño del LEL y el de los Escenarios derivados de él, permitiendo anticipar características de los Escenarios a partir de medir el $\mathcal{L E} \mathcal{L}$.

Relaciones con otras mediciones

Vincular el tamaño del LEL, y potencialmente de los escenarios, con otras medidas de tamaño de productos y de funcionalidad de escenarios y otros artefactos. 
[Antonelli '00] Antonelli L.; "Traceability en Requirements Baseline y Baseline Mentor Workbench (BMW)" Jornadas sobre ingeniería de requisitos. Universidad De Belgrano, 1 de Diciembre del 2000.

[Antonelli '01] Antonelli, L., Oliveros, A., "Traceability en la etapa de elicitación de requerimientos" en Proceedings del IV Workshop on Requirements Engine ring, $U \mathcal{T} \mathcal{N}$ - noviembre 22 y 23, 2001.

[Briand 96] Briand, Lionel C., Morasaca, Sandro, Basili, Victor, "Property-Based Software Engine ering Measure ment" - IEEE Transactions on S of tware Engine ering Vol. 22 No. 1, I anuary 1996

[Briand 98] Briand, Lionel C., Wüst, Jürgen, "Integrating Scenario-based and Measurement-6ased Software Product Assessment" - IESEReport №. $042.00 / \mathcal{E}$ - IS ERN Re port $\mathcal{N}$. IS ERN-00-04

[Berenson 96] Berenson, Mark; Levine, David; "Estadística básica en administración", Prentice-Hall His panoame ricana, S.A., 1996

[Christel'93] Christel, Michael G., Wood, David P., S tevens, Scott M., "AMORE: The Advanced Multimedia Organizer for Requirements Elicitation" Copyright (C) 1993 by Carnegie Mellon University.

[Doorn 98] Doorn, g., Kaplan, G., Hadad, G., Leite, g.C.S.P., "Inspección de Escenarios", in Proceedings of Workshop on Requirements Engineering, Maringa, Brasil, 1998 .

[Enc. Océano 90] Enciclopedia Temática Océano, Volumen I, "Gramática Española", Grupo Editorial Océano, 1990

[Enc. Labor '62] Enciclopedia Labor, Volumen VI, "El lenguaje y las matemáticas", Editorial Labor, 1962

[Exce['00] Microsoft Excel 2000

[Faloutsos 95] Faloutsos, Cristos, Orad, Douglas W., " $\mathcal{A}$ S urvey of Information Retrieval and Filtering Methods" Department of Computer Science, Unive rsity of Maryland

[Fenton 96] Fenton, Norman E., Pfleeger, Shari L. "Software Metrics: A Rigorous of Practical Approach", International Thomson Computer Press, London, UK. 1996 
[García '00] García, Omar F., Gentile, Claudio G., "Análisis de requisitos al $\mathcal{L E L}$ (Léxico Extendido del Lenguaje) y a los Escenarios, con la me todología que se basa en el uso de LEL y escenarios ", Página en Internet, http://usuarios.arnet.com.ar/ogarcia/

[Grady 94] Grady, Robert B.; "Successfully Applying Software Metrics" , IEEE, Computer, Septiembre 1994

[Guarin 02] $\mathcal{N}$. Guarin $S$, "Estadístic a aplicada, 7 ". Medidas de dispersión. Página en Internet.

[Humphrey 90] Humprey, W.S., "Managing the Software Process", Addison Wesley, 1990

[Hadad 97] Hadad, G., Kaplan, G., Oliveros, A., Leite, J.C.S.P., "Construción de Escenarios a partir del Léxico Extendido del Lenguaje" IAIIO'97, $S \mathcal{A D I O} \mathcal{B}$ ue nos Aires, $1997, \mathrm{pp} .65-77$

[Hammer '97] Hammer, $\mathcal{T} .$, Rosenberg, L., Huffman, L., Hyatt, L., "Measuring Requirements $\mathcal{T}$ esting", ICSE 97 Boston $\mathcal{M A}$ US A, 1997

[Jackson '95] Jackson, M., "Software Requirements e' Specifications", Addison Wesley, 1995

[Jofnsonbaugh '88] Johnsonbaugh, R., "Matemáticas Discretas", Grupo Editorial I beroamérica, 1988

[Jones 95] Jones, G., Robertson, $\mathcal{A}$. M., Santimetvirul, C., Willet, P., "Nonfierarchical document clustering using a gene tic algorithm", De patment of Information $S$ tudies, University of $S$ he ffield, UK 1995

[Kaplan 96] Kaplan, G., Oliveros, A., Leite, J.C.S.P, "Integración de escenarios con el $\mathcal{L E L}$ en la elicitación de requerimientos: aplicación a un caso re al". Mayo 1996

[Lagunilla 95] Lagunilla, Marina Fernández, Rebollo, A. Anula, "S intaxis y cognición. Introducción al conocimiento, el procesamiento y los déficits sintácticos", Editorial $\mathcal{S}$ intes is, S. A.,1995.

[Leite 96] Leite, J.C.S.P., Oliveros, A., Rossi, G., Balaguer, F., Hadad, G., V., Kaplan, Maiorana, G., "Léxico extendido del lenguaje y escenarios del sistema nacional para la obtención de pasaportes", Documento de Trabajo $\mathcal{N}$ ro. 3 , Universidad de Belgrano, Departamento de Investigación, 1996.

[Leite '97] Leite, J.C.S.P., Rossi, G., Balaguer, F., Maiorana, V., Kaplan, G., Hadad, G., Oliveros, $\mathcal{A}$., "Enflancing a Requirement Baseline with Scenarios", in 
Proce edings of the Third IEEE International Symposium on Require ments Engine ring, I EEE Computer Society Press, Los Alamitos, 1997.

[Leite '00] Leite, J.C.S.P., Hadad, G., Doorn, J., Kaplan, G., "A Scenario Construction Process ", Requirements Engine ering Iournal, 2000

[Le Vie '00] Le Vie Ir., Donald S.; "Metrics: What Do You Re ally Want To Me asure?", Revista Intercom

[Levin '96] Levin, Richard; Rubin, David; "Estadistic a para administradores", Prentice $\mathcal{H a l l} \mathcal{H}$ is panoame ric ana, $S$.A., 1996

[Loucopoulos 95] Loucopoulos, P.; Karakostas, V.; "System Requirements Engine ering", Mc Graw-Hill, London, 1995

[Marin '82] "Diccionario Marín de la Lengua Española", Ed. Marin S.A., 1982

[Martinez'99] Martinez Mercado, D. E. "Software Engineering", página en Internet, 1999

[Mills '8 8] Mills, E.E., "S oftware Metrics", SEI Curriculum Module SEI-CM-12-1.1, 1988

[Morasca '99] Morasca, Sandro; "Measuring Attributes of Concurrent Software Specifications in Petri Nets", Sixth IEEE Intl. Symposium on Software Metrics, Boca Raton, Florida, November 04-06, 1999

[Nuseibef '97] $\mathcal{N u s e i b e h , ~ B a s h a r ; ~ R o b e r t s o n , ~ S . ; ~ " M a k i n g ~ R e q u i r e m e n t s ~ M e a s u r a b l e " , ~}$ paper en Internet

[Offen '97] Offen, Raymond I., I effery, Ross, "Establishing Software Me asure ment Programs" I EEE $S$ of tware, 1997

[Pressman 93] Pressman, R.S., "Ingeniería del Software. Un enfoque práctico", $\mathcal{M c G r a w - H i l l , ~ M a d r i d , ~} 1993$

[Robertson 97] Robertson, I., Robertson, S.; "Requirements: Made to Measure", Paper en Internet, 1997

[Rule '01] Rule, P. Grant, "The Importance of the Size of Software Requirements", Presentado en la Conferencia $\mathcal{N A S S C O M}$, Hotel Oberoi Towers, Mumbai, India, $7-10 \mathcal{F e}$ brero 2001

[Sopena 63] "Enciclopedia Universal Sopena", Ed. Ramón Sopena, 1963 
$[S$ utcliffe '97] $\mathcal{S}$ utcliffe, Alistair "Exploring $\mathcal{S}$ cenarios in Require ments Engine ering", paper of Workshop

[Van Rijsbergen "79] Van Rijsbergen, C. I., "Information Retrieval" Butterworths, London, 2nd Edition, 1979

[Wilson 99] Wilson, William "Writing Effective Natural Languaje Requirements Specifications", Paper en Internet, febrero 1999

[Wilson 97] Wilson, William $\mathcal{M}$., Rosenberg, Linda H., Hyatt, Laurence $\mathcal{E}$. "Automated Analysis of Requirement $S$ pecifications". Paper en Internet, mayo 1997

[Yourdon 79] Yourdon, E., Constantine, L.L., "Structured Design. Fundamentals of a Discipline of Computer Program and System Design", Prentice-Hall, 1979

[Zervas 00] Zervas, Giorgio, Ruger, Stefan, "The curse of dimensionality and document clustering", Department of Computer Science, University of Minnesota, 2000

[Zuse 95] Zuse, Horst; "History of Software Measurement", http://irb.cs.tuberlin.de/ zuse/metrics/3-hist.html, 1995 
APENDICE F.1

\section{MEDICIONES APLICADAS AL SIMBOLO DEL LEL HOTEL}

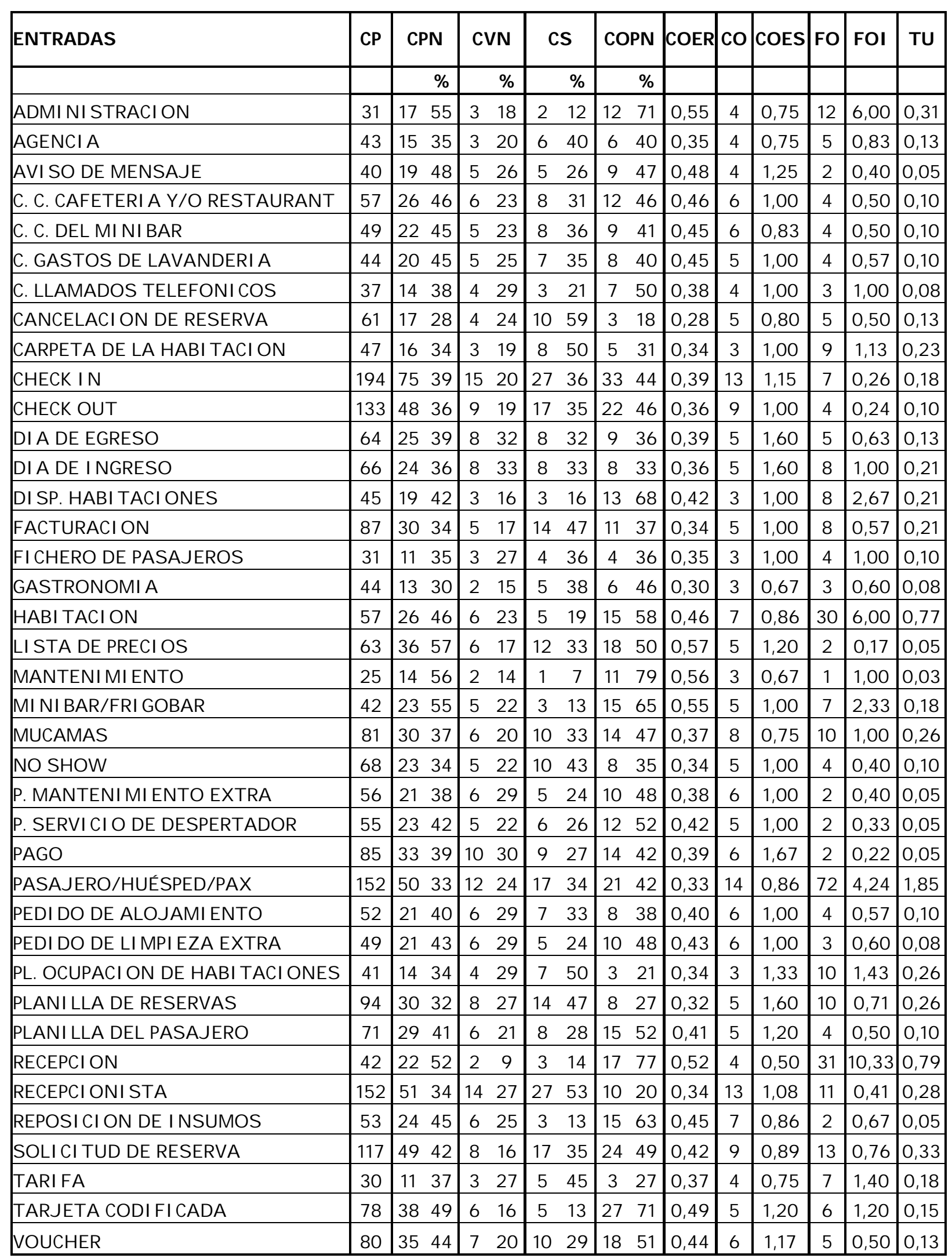




\section{MEDICIONES APLICADAS AL LEL "HOTEL"}

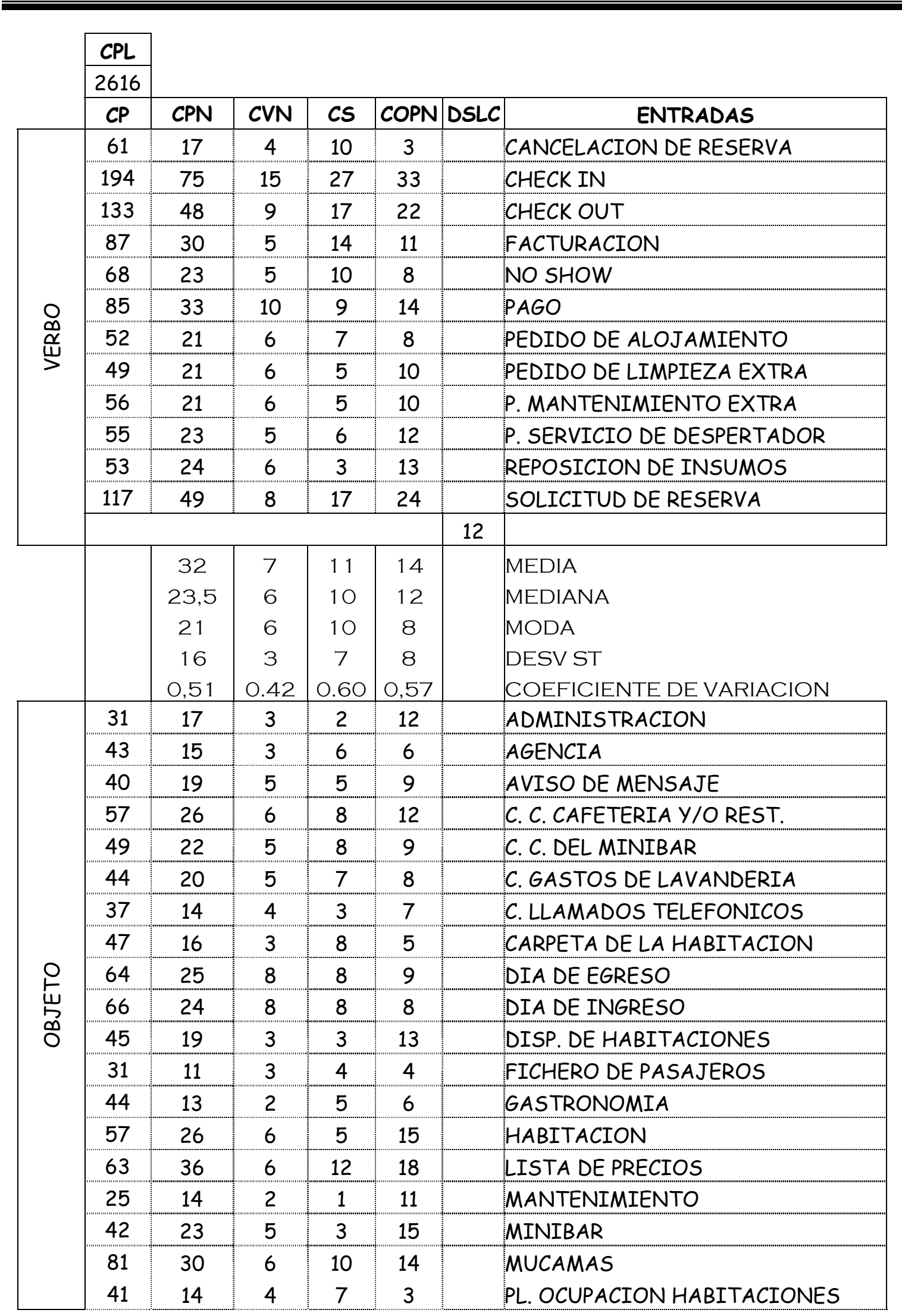




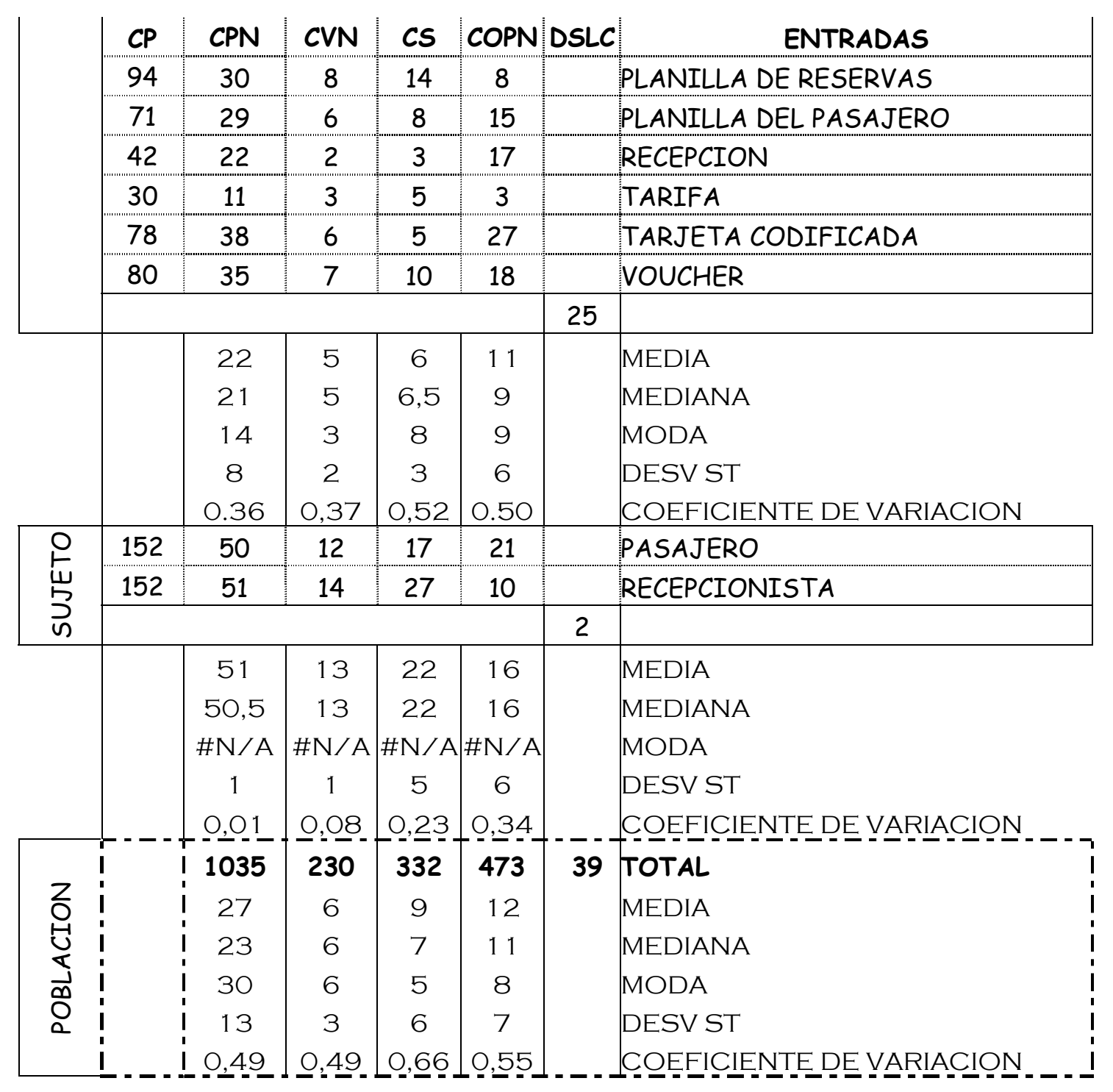


APENDICE F.3

MATRIZ DE ADYACENCIA PONDERADA DEL LEL "HOTEL" 


\begin{tabular}{|c|c|c|c|c|c|c|c|c|c|c|c|c|c|c|c|c|c|c|c|c|c|c|}
\hline & ADM. & AGENCIA & $\begin{array}{l}\text { AVISO } \\
\text { MENS. }\end{array}$ & $\begin{array}{l}\text { C. GAST. } \\
\text { LAV. }\end{array}$ & C. LL. TEL. & $\begin{array}{l}\text { C.C.C.CAF. } \\
\text { RESTT. }\end{array}$ & \begin{tabular}{|l} 
C.C. \\
MINBAR
\end{tabular} & $\begin{array}{c}\text { CANC. } \\
\text { RESERVA }\end{array}$ & $\begin{array}{l}\text { CARP. } \\
\text { HAB. }\end{array}$ & CHECK IN & $\begin{array}{c}\text { CHECK } \\
\text { OUT }\end{array}$ & COMPRAS & $\begin{array}{c}\text { DIA } \\
\text { EGRESO }\end{array}$ & $\begin{array}{c}\text { DIA } \\
\text { INGRESO }\end{array}$ & $\begin{array}{l}\text { DISP. } \\
\text { HAB. }\end{array}$ & FACT. & FICH. PAS. & GASTR. & GERENCIA & HAB. & $\begin{array}{l}\text { LISTA } \\
\text { PRECIOS }\end{array}$ & MANT. \\
\hline 定 & 0 & 0 & 0 & 0 & 0 & 0 & 0 & 0 & 0 & 0 & 0 & 0 & 0 & 0 & 0 & 1 & 0 & 0 & 0 & 0 & 0 & 0 \\
\hline 壳 & 0 & 0 & 0 & 0 & 0 & 0 & 0 & 1 & 0 & 0 & 0 & 0 & 0 & 0 & 0 & 0 & 0 & 0 & 0 & 0 & 0 & 0 \\
\hline 品总 & 0 & 0 & 0 & 0 & 0 & 0 & 0 & 0 & 0 & 0 & 0 & 0 & 0 & 0 & 0 & 0 & 0 & 0 & 0 & 1 & 0 & 0 \\
\hline 悹字 & 0 & 0 & 0 & 0 & 0 & 0 & 0 & 0 & 1 & 0 & 0 & 0 & 0 & 0 & 0 & 0 & 0 & 0 & 0 & 1 & 0 & 0 \\
\hline نُّن & 0 & 0 & 0 & 0 & 0 & 0 & 0 & 0 & 1 & 0 & 0 & 0 & 0 & 0 & 0 & 0 & 0 & 0 & 0 & 1 & 0 & 0 \\
\hline 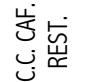 & 0 & 0 & 0 & 0 & 0 & 0 & 0 & 0 & 1 & 0 & 0 & 0 & 0 & 0 & 0 & 0 & 0 & 3 & 0 & 1 & 0 & 0 \\
\hline ن ن & 0 & 0 & 0 & 0 & 0 & 0 & 0 & 0 & 1 & 0 & 0 & 0 & 0 & 0 & 0 & 0 & 0 & 0 & 0 & 1 & 0 & 0 \\
\hline 总㺃 & 0 & 1 & 0 & 0 & 0 & 0 & 0 & 0 & 0 & 0 & 0 & 0 & 0 & 0 & 1 & 0 & 0 & 0 & 0 & 0 & 0 & 0 \\
\hline 酸兽 & 1 & 0 & 0 & 1 & 1 & 1 & 1 & 0 & 0 & 0 & 0 & 0 & 0 & 0 & 0 & 1 & 0 & 0 & 0 & 0 & 0 & 0 \\
\hline 站 & 0 & 0 & 0 & 0 & 0 & 0 & 0 & 0 & 1 & 0 & 0 & 0 & 0 & 0 & 1 & 0 & 2 & 0 & 0 & 4 & 0 & 0 \\
\hline 总与 & 1 & 0 & 0 & 1 & 1 & 1 & 1 & 0 & 0 & 0 & 0 & 0 & 0 & 0 & 1 & 0 & 0 & 0 & 0 & 0 & 0 & 0 \\
\hline 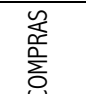 & 0 & 0 & 0 & 0 & 0 & 0 & 0 & 0 & 0 & 0 & 0 & 0 & 0 & 0 & 0 & 0 & 0 & 0 & 0 & 0 & 0 & 0 \\
\hline 言总 & 1 & 0 & 0 & 0 & 0 & 0 & 0 & 0 & 0 & 0 & 0 & 0 & 0 & 1 & 0 & 1 & 0 & 0 & 0 & 0 & 0 & 0 \\
\hline 道 & 1 & 0 & 0 & 0 & 0 & 0 & 0 & 0 & 0 & 0 & 0 & 0 & 1 & 0 & 0 & 1 & 0 & 0 & 0 & 0 & 0 & 0 \\
\hline
\end{tabular}




\begin{tabular}{|c|c|c|c|c|c|c|c|c|c|c|c|c|c|c|c|c|c|c|c|c|c|c|}
\hline & ADM. & AGENCIA & $\begin{array}{l}\text { AVISO } \\
\text { MENS. }\end{array}$ & $\begin{array}{l}\text { C. GAST, } \\
\text { LAV. }\end{array}$ & C. LL. TEL. & \begin{tabular}{|c} 
c.C. CAF. \\
REST.
\end{tabular} & \begin{tabular}{|c} 
c.C. \\
MINIBAR \\
\end{tabular} & $\begin{array}{l}\text { CANC. } \\
\text { RESERV. }\end{array}$ & $\begin{array}{l}\text { CARP. } \\
\text { HAB. }\end{array}$ & CHECK IN & CHECK OU & TCOMPRAS & \begin{tabular}{|c|} 
DIA \\
EGRESO
\end{tabular} & $\begin{array}{c}\text { DIA } \\
\text { INGRESO }\end{array}$ & DISP. HAB, & FACT. & FICH. PAS. & GASTR. & GERENCIA & HAB. & $\begin{array}{l}\text { LISTA } \\
\text { PRECIOS }\end{array}$ & MANT. \\
\hline 产离 & 0 & 0 & 0 & 0 & 0 & 0 & 0 & 0 & 0 & 0 & 0 & 0 & 0 & 0 & 0 & 0 & 0 & 0 & 0 & 1 & 0 & 0 \\
\hline 氨 & 3 & 0 & 0 & 0 & 0 & 0 & 0 & 0 & 1 & 0 & 0 & 0 & 2 & 2 & 0 & 0 & 0 & 0 & 0 & 2 & 0 & 0 \\
\hline 픈 & 0 & 0 & 0 & 0 & 0 & 0 & 0 & 0 & 0 & 1 & 0 & 0 & 0 & 0 & 0 & 0 & 0 & 0 & 0 & 0 & 0 & 0 \\
\hline 离 & 0 & 0 & 0 & 0 & 0 & 1 & 0 & 0 & 0 & 0 & 0 & 0 & 0 & 0 & 0 & 0 & 0 & 0 & 0 & 1 & 0 & 0 \\
\hline 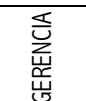 & 0 & 0 & 0 & 0 & 0 & 0 & 0 & 0 & 0 & 0 & 0 & 0 & 0 & 0 & 0 & 0 & 0 & 0 & 0 & 0 & 0 & 0 \\
\hline$\frac{\dot{q}}{x}$ & 0 & 0 & 0 & 0 & 0 & 0 & 0 & 0 & 0 & 1 & 1 & 0 & 0 & 0 & 0 & 0 & 0 & 0 & 0 & 0 & 0 & 0 \\
\hline 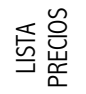 & 2 & 0 & 0 & 0 & 0 & 0 & 0 & 0 & 0 & 0 & 0 & 0 & 0 & 0 & 0 & 0 & 0 & 0 & 1 & 3 & 0 & 0 \\
\hline$\sum_{\frac{1}{\Sigma}}^{+}$ & 0 & 0 & 0 & 0 & 0 & 0 & 0 & 0 & 0 & 0 & 0 & 0 & 0 & 0 & 0 & 0 & 0 & 0 & 0 & 1 & 0 & 0 \\
\hline 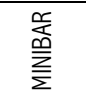 & 0 & 0 & 0 & 0 & 0 & 0 & 0 & 0 & 0 & 0 & 0 & 0 & 0 & 0 & 0 & 0 & 0 & 0 & 0 & 1 & 0 & 0 \\
\hline $\begin{array}{l}\text { 荇 } \\
\text { 妾 }\end{array}$ & 0 & 0 & 0 & 1 & 0 & 0 & 1 & 0 & 0 & 0 & 0 & 0 & 0 & 0 & 0 & 0 & 0 & 0 & 0 & 1 & 0 & 0 \\
\hline २혹 & 0 & 0 & 0 & 0 & 0 & 0 & 0 & 0 & 0 & 0 & 0 & 0 & 0 & 1 & 1 & 0 & 0 & 0 & 0 & 0 & 0 & 0 \\
\hline $\begin{array}{l}\text { 密 } \\
\text { 焉 }\end{array}$ & 0 & 0 & 0 & 0 & 0 & 0 & 0 & 0 & 0 & 0 & 0 & 0 & 0 & 0 & 0 & 0 & 0 & 0 & 0 & 1 & 0 & 0 \\
\hline 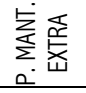 & 0 & 0 & 0 & 0 & 0 & 0 & 0 & 0 & 0 & 0 & 0 & 0 & 0 & 0 & 0 & 0 & 0 & 0 & 0 & 1 & 0 & 1 \\
\hline 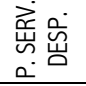 & 0 & 0 & 0 & 0 & 0 & 0 & 0 & 0 & 0 & 0 & 0 & 0 & 0 & 0 & 0 & 0 & 0 & 0 & 0 & 2 & 0 & 0 \\
\hline 过 & 1 & 0 & 0 & 0 & 0 & 0 & 0 & 0 & 0 & 0 & 0 & 0 & 0 & 0 & 0 & 1 & 0 & 0 & 0 & 0 & 0 & 0 \\
\hline
\end{tabular}




\begin{tabular}{|c|c|c|c|c|c|c|c|c|c|c|c|c|c|c|c|c|c|c|c|c|c|c|}
\hline & ADM. & AGENCIA & $\begin{array}{l}\text { AVISO } \\
\text { MENS. }\end{array}$ & $\begin{array}{c}\text { C. GAST, } \\
\text { LAV. }\end{array}$ & C. LL. TEL. & \begin{tabular}{|c|} 
c.C. CAF. \\
REST. \\
\end{tabular} & \begin{tabular}{|c|} 
C.C. \\
MINIBAR \\
\end{tabular} & \begin{tabular}{|c} 
CANC. \\
RESERV. \\
\end{tabular} & $\begin{array}{l}\text { CARP. } \\
\text { HAB. }\end{array}$ & CHECK IN & $\begin{array}{c}\text { CHECK } \\
\text { OUT }\end{array}$ & COMPRAS & \begin{tabular}{|c} 
DIA \\
EGRESO \\
\end{tabular} & $\begin{array}{c}\text { DIA } \\
\text { INGRESO }\end{array}$ & $\begin{array}{l}\text { DISP. } \\
\text { HAB, }\end{array}$ & FACT. & FICH. PAS. & GASTR. & GERENCIA & HAB. & $\begin{array}{c}\text { LISTA } \\
\text { PRECIOS }\end{array}$ & MANT. \\
\hline$\frac{\dot{2}}{2}$ & 0 & 0 & 1 & 0 & 0 & 0 & 0 & 1 & 0 & 0 & 0 & 0 & 0 & 0 & 0 & 0 & 0 & 0 & 0 & 1 & 0 & 0 \\
\hline 竒完 & 0 & 0 & 0 & 0 & 0 & 0 & 0 & 0 & 0 & 1 & 0 & 0 & 0 & 0 & 1 & 0 & 0 & 0 & 0 & 1 & 0 & 0 \\
\hline 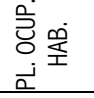 & 0 & 0 & 0 & 0 & 0 & 0 & 0 & 1 & 0 & 1 & 1 & 0 & 0 & 0 & 0 & 0 & 0 & 0 & 0 & 1 & 0 & 0 \\
\hline 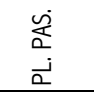 & 0 & 0 & 0 & 0 & 0 & 0 & 0 & 0 & 0 & 1 & 0 & 0 & 0 & 1 & 0 & 0 & 2 & 0 & 0 & 0 & 0 & 0 \\
\hline ¿ & 0 & 0 & 0 & 0 & 0 & 0 & 0 & 1 & 0 & 1 & 1 & 0 & 0 & 0 & 0 & 0 & 0 & 0 & 0 & 1 & 0 & 0 \\
\hline 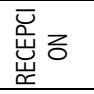 & 0 & 0 & 0 & 0 & 0 & 0 & 0 & 0 & 0 & 0 & 0 & 0 & 0 & 0 & 0 & 0 & 0 & 0 & 0 & 0 & 0 & 0 \\
\hline $\begin{array}{l}\text { 总 } \\
\text { 点 }\end{array}$ & 1 & 0 & 1 & 1 & 1 & 1 & 1 & 1 & 2 & 1 & 1 & 0 & 0 & 0 & 1 & 1 & 0 & 0 & 0 & 0 & 0 & 0 \\
\hline 咅总总 & 0 & 0 & 0 & 0 & 0 & 0 & 0 & 0 & 0 & 0 & 0 & 2 & 0 & 0 & 0 & 0 & 0 & 0 & 0 & 0 & 0 & 0 \\
\hline 方胥 & 0 & 2 & 0 & 0 & 0 & 0 & 0 & 0 & 0 & 0 & 0 & 0 & 1 & 2 & 2 & 0 & 0 & 0 & 0 & 1 & 0 & 0 \\
\hline $\begin{array}{l}\text { 委 } \\
\text { 年 } \\
\end{array}$ & 1 & 0 & 0 & 0 & 0 & 0 & 0 & 0 & 0 & 0 & 0 & 0 & 0 & 0 & 0 & 1 & 0 & 0 & 0 & 1 & 2 & 0 \\
\hline 定宅 & 0 & 0 & 0 & 0 & 0 & 0 & 0 & 0 & 0 & 0 & 0 & 0 & 0 & 0 & 0 & 0 & 0 & 0 & 0 & 1 & 0 & 0 \\
\hline $\begin{array}{l}\text { 喜 } \\
\stackrel{亏}{9}\end{array}$ & 0 & 2 & 0 & 0 & 0 & 0 & 0 & 0 & 1 & 0 & 0 & 0 & 1 & 1 & 0 & 1 & 0 & 0 & 0 & 0 & 0 & 0 \\
\hline$\frac{2}{4} 5$ & 12 & 5 & 2 & 4 & 3 & 4 & 4 & 5 & 9 & 7 & 4 & 2 & 5 & 8 & 8 & 8 & 4 & 3 & 1 & 30 & 2 & 1 \\
\hline
\end{tabular}




\begin{tabular}{|c|c|c|c|c|c|c|c|c|c|c|c|c|c|c|c|c|c|c|c|c|}
\hline & MINIBAR & MUCAMAS & No SHow & $\begin{array}{l}\text { P. LIMP. } \\
\text { EXTRA }\end{array}$ & $\begin{array}{c}\text { P. MANT. } \\
\text { EXTRA }\end{array}$ & $\begin{array}{c}\text { P. SERV. } \\
\text { DESP. }\end{array}$ & PAGO & PAS. & $\begin{array}{l}\text { PED. } \\
\text { ALOJ. }\end{array}$ & $\begin{array}{c}\begin{array}{c}\text { PL. OCUP. } \\
\text { HAB. }\end{array} \\
\end{array}$ & PL. PAS. & \begin{tabular}{c|} 
PL. \\
RESERV.
\end{tabular} & RECEPCION & $\begin{array}{c}\text { RECEPCIONIS } \\
\text { TA }\end{array}$ & $\begin{array}{l}\text { REP. } \\
\text { INSUMOS }\end{array}$ & $\begin{array}{c}\text { SOL. } \\
\text { RESERVA }\end{array}$ & TARIFA & TARJ. COD. & VOUCHER & $\begin{array}{c}\text { FAN } \\
\text { IN }\end{array}$ \\
\hline 妾 & 0 & 0 & 0 & 0 & 0 & 0 & 0 & 1 & 0 & 0 & 0 & 0 & 0 & 0 & 0 & 0 & 0 & 0 & 0 & 2 \\
\hline 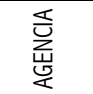 & 0 & 0 & 0 & 0 & 0 & 0 & 0 & 3 & 0 & 0 & 0 & 0 & 0 & 0 & 0 & 1 & 0 & 0 & 1 & 6 \\
\hline 酸盖 & 0 & 0 & 0 & 0 & 0 & 0 & 0 & 2 & 0 & 0 & 0 & 0 & 2 & 0 & 0 & 0 & 0 & 0 & 0 & 5 \\
\hline 葸方 & 0 & 3 & 0 & 0 & 0 & 0 & 0 & 1 & 0 & 0 & 0 & 0 & 1 & 0 & 0 & 0 & 0 & 0 & 0 & 7 \\
\hline نُّنَّن & 0 & 0 & 0 & 0 & 0 & 0 & 0 & 1 & 0 & 0 & 0 & 0 & 0 & 0 & 0 & 0 & 0 & 0 & 0 & 3 \\
\hline 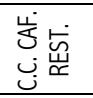 & 0 & 0 & 0 & 0 & 0 & 0 & 0 & 2 & 0 & 0 & 0 & 0 & 1 & 0 & 0 & 0 & 0 & 0 & 0 & 8 \\
\hline ن & 1 & 3 & 0 & 0 & 0 & 0 & 0 & 1 & 0 & 0 & 0 & 0 & 1 & 0 & 0 & 0 & 0 & 0 & 0 & 8 \\
\hline 鸢密 & 0 & 0 & 0 & 0 & 0 & 0 & 0 & 3 & 0 & 1 & 0 & 1 & 1 & 0 & 0 & 2 & 0 & 0 & 0 & 10 \\
\hline 酸案 & 0 & 0 & 0 & 0 & 0 & 0 & 0 & 0 & 0 & 0 & 0 & 0 & 1 & 0 & 0 & 0 & 0 & 0 & 1 & 8 \\
\hline 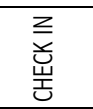 & 1 & 0 & 0 & 0 & 0 & 0 & 0 & 6 & 0 & 1 & 2 & 2 & 1 & 3 & 0 & 0 & 0 & 2 & 1 & 27 \\
\hline 酸氙 & 1 & 0 & 0 & 0 & 0 & 0 & 1 & 5 & 0 & 1 & 0 & 0 & 1 & 1 & 0 & 0 & 0 & 1 & 0 & 17 \\
\hline 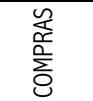 & 0 & 0 & 0 & 0 & 0 & 0 & 0 & 0 & 0 & 0 & 0 & 0 & 0 & 0 & 1 & 0 & 0 & 0 & 0 & 1 \\
\hline 言惫 & 0 & 0 & 0 & 0 & 0 & 0 & 0 & 3 & 0 & 0 & 0 & 1 & 0 & 1 & 0 & 0 & 0 & 0 & 0 & 8 \\
\hline 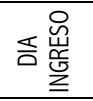 & 0 & 0 & 0 & 0 & 0 & 0 & 0 & 3 & 0 & 0 & 0 & 1 & 0 & 1 & 0 & 0 & 0 & 0 & 0 & 8 \\
\hline 商 $\frac{\ddot{q}}{x}$ & 0 & 0 & 0 & 0 & 0 & 0 & 0 & 0 & 0 & 2 & 0 & 0 & 0 & 0 & 0 & 0 & 0 & 0 & 0 & 3 \\
\hline
\end{tabular}




\begin{tabular}{|c|c|c|c|c|c|c|c|c|c|c|c|c|c|c|c|c|c|c|c|c|}
\hline & MINIBAR & MUCAMAS & No SHOW & $\begin{array}{l}\text { P. LIMP. } \\
\text { EXTRA }\end{array}$ & $\begin{array}{c}\text { P. MANT. } \\
\text { EXTRA }\end{array}$ & $\begin{array}{l}\text { P. SERV. } \\
\text { DESP. }\end{array}$ & PAGO & PAS. & $\begin{array}{l}\text { PED. } \\
\text { ALOJ. }\end{array}$ & $\begin{array}{l}\text { PL. OCUP. } \\
\text { HAB. }\end{array}$ & PL. PAS. & $\begin{array}{c}\text { PL. } \\
\text { RESERV. }\end{array}$ & RECEPCION & $\begin{array}{c}\text { RECEPCIONIS } \\
\text { TA }\end{array}$ & \begin{tabular}{|c|} 
REP. \\
INSUMOS
\end{tabular} & $\begin{array}{c}\text { SOL. } \\
\text { RESERVA }\end{array}$ & TARIFA & TARJ. COD. & VOUCHER & $\begin{array}{l}\text { FAN } \\
\text { IN }\end{array}$ \\
\hline 崖 & 0 & 0 & 0 & 0 & 0 & 0 & 0 & 3 & 0 & 0 & 0 & 1 & 0 & 0 & 0 & 0 & 0 & 0 & 0 & 14 \\
\hline 폰롱 & 0 & 0 & 0 & 0 & 0 & 0 & 0 & 1 & 0 & 0 & 1 & 0 & 1 & 0 & 0 & 0 & 0 & 0 & 0 & 4 \\
\hline 竞 & 0 & 0 & 0 & 0 & 0 & 0 & 0 & 1 & 0 & 0 & 0 & 0 & 1 & 0 & 0 & 0 & 1 & 0 & 0 & 5 \\
\hline 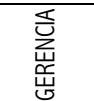 & 0 & 0 & 0 & 0 & 0 & 0 & 0 & 0 & 0 & 0 & 0 & 0 & 0 & 0 & 0 & 0 & 0 & 0 & 0 & 0 \\
\hline$\frac{\ddot{q}}{x}$ & 0 & 0 & 0 & 0 & 0 & 0 & 0 & 1 & 0 & 0 & 0 & 0 & 0 & 0 & 0 & 1 & 1 & 0 & 0 & 5 \\
\hline 点总 & 0 & 0 & 0 & 0 & 0 & 0 & 0 & 0 & 1 & 0 & 0 & 0 & 2 & 0 & 0 & 1 & 3 & 0 & 0 & 13 \\
\hline$\sum_{\frac{1}{2}}^{+}$ & 0 & 0 & 0 & 0 & 0 & 0 & 0 & 0 & 0 & 0 & 0 & 0 & 0 & 0 & 0 & 0 & 0 & 0 & 0 & 1 \\
\hline 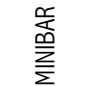 & 0 & 1 & 0 & 0 & 0 & 0 & 0 & 1 & 0 & 0 & 0 & 0 & 0 & 0 & 0 & 0 & 0 & 0 & 0 & 3 \\
\hline 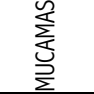 & 1 & 0 & 0 & 1 & 0 & 0 & 0 & 2 & 0 & 0 & 0 & 0 & 2 & 0 & 0 & 0 & 0 & 1 & 0 & 10 \\
\hline 을 홍 & 0 & 0 & 0 & 0 & 0 & 0 & 0 & 2 & 0 & 1 & 0 & 1 & 1 & 1 & 0 & 2 & 0 & 0 & 0 & 10 \\
\hline 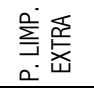 & 0 & 1 & 0 & 0 & 0 & 0 & 0 & 2 & 0 & 0 & 0 & 0 & 1 & 0 & 0 & 0 & 0 & 0 & 0 & 5 \\
\hline 案趇 & 0 & 0 & 0 & 0 & 0 & 0 & 0 & 2 & 0 & 0 & 0 & 0 & 1 & 0 & 0 & 0 & 0 & 0 & 0 & 5 \\
\hline $\begin{array}{l}\text { 离怘 } \\
\text { a. }\end{array}$ & 0 & 0 & 0 & 0 & 0 & 0 & 0 & 3 & 0 & 0 & 0 & 0 & 1 & 0 & 0 & 0 & 0 & 0 & 0 & 6 \\
\hline 웅 & 0 & 0 & 0 & 0 & 0 & 0 & 0 & 4 & 0 & 0 & 0 & 0 & 3 & 0 & 0 & 0 & 0 & 0 & 0 & 9 \\
\hline 定 & 3 & 0 & 0 & 1 & 1 & 1 & 1 & 0 & 1 & 0 & 1 & 0 & 0 & 1 & 0 & 1 & 0 & 2 & 1 & 17 \\
\hline 这运 & 0 & 0 & 0 & 0 & 0 & 0 & 0 & 0 & 0 & 1 & 0 & 1 & 1 & 0 & 0 & 1 & 0 & 0 & 0 & 7 \\
\hline
\end{tabular}




\begin{tabular}{|c|c|c|c|c|c|c|c|c|c|c|c|c|c|c|c|c|c|c|c|c|}
\hline & MINIBAR & MUCAMAS & No SHOW & $\begin{array}{l}\text { P. LIMP. } \\
\text { EXTRA }\end{array}$ & $\begin{array}{c}\text { P. MANT. } \\
\text { EXTRA }\end{array}$ & $\begin{array}{l}\text { P. SERV. } \\
\text { DESP. }\end{array}$ & PAGO & PAS. & $\begin{array}{l}\text { PED. } \\
\text { ALOJ. }\end{array}$ & $\begin{array}{l}\text { PL. OCUP. } \\
\text { HAB. }\end{array}$ & PL. PAS. & $\begin{array}{c}\text { PL. } \\
\text { RESERV. }\end{array}$ & RECEPCION & $\begin{array}{c}\text { RECEPCIONIS } \\
\text { TA }\end{array}$ & \begin{tabular}{|c|} 
REP. \\
INSUMOS
\end{tabular} & $\begin{array}{l}\text { SOL. } \\
\text { RESERVA }\end{array}$ & TARIFA & TARJ. COD. & VOUCHER & $\begin{array}{c}\text { FAN } \\
\text { IN }\end{array}$ \\
\hline 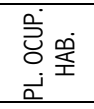 & 0 & 0 & 1 & 0 & 0 & 0 & 0 & 0 & 0 & 0 & 0 & 0 & 1 & 0 & 0 & 1 & 0 & 0 & 0 & 7 \\
\hline 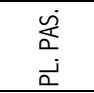 & 0 & 0 & 0 & 0 & 0 & 0 & 0 & 3 & 0 & 0 & 0 & 0 & 1 & 0 & 0 & 0 & 0 & 0 & 0 & 8 \\
\hline d竞 & 0 & 0 & 1 & 0 & 0 & 0 & 0 & 4 & 1 & 1 & 0 & 0 & 1 & 0 & 0 & 2 & 0 & 0 & 0 & 14 \\
\hline $\begin{array}{l}\overline{\bar{u}} \\
\text { 峞z }\end{array}$ & 0 & 0 & 0 & 0 & 0 & 0 & 0 & 2 & 0 & 0 & 0 & 0 & 0 & 1 & 0 & 0 & 0 & 0 & 0 & 3 \\
\hline 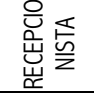 & 0 & 0 & 1 & 1 & 1 & 1 & 0 & 3 & 1 & 0 & 0 & 1 & 2 & 0 & 1 & 1 & 0 & 0 & 1 & 27 \\
\hline 兽离 & 0 & 0 & 0 & 0 & 0 & 0 & 0 & 0 & 0 & 0 & 0 & 0 & 2 & 1 & 0 & 0 & 0 & 0 & 0 & 5 \\
\hline 定䞷 & 0 & 0 & 1 & 0 & 0 & 0 & 0 & 2 & 0 & 2 & 0 & 1 & 1 & 0 & 0 & 0 & 2 & 0 & 0 & 17 \\
\hline 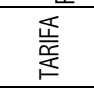 & 0 & 0 & 0 & 0 & 0 & 0 & 0 & 0 & 0 & 0 & 0 & 0 & 0 & 0 & 0 & 0 & 0 & 0 & 0 & 5 \\
\hline 롫 & 0 & 2 & 0 & 0 & 0 & 0 & 0 & 2 & 0 & 0 & 0 & 0 & 0 & 0 & 0 & 0 & 0 & 0 & 0 & 5 \\
\hline 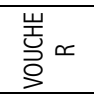 & 0 & 0 & 0 & 0 & 0 & 0 & 0 & 3 & 0 & 0 & 0 & 0 & 0 & 1 & 0 & 0 & 0 & 0 & 0 & 10 \\
\hline$\frac{2}{4} 5$ & 7 & 10 & 4 & 3 & 2 & 2 & 2 & 72 & 4 & 10 & 4 & 10 & 31 & 11 & 2 & 13 & 7 & 6 & 5 & \\
\hline
\end{tabular}


APENDICE G.1

\section{MEDICIONES APLICADAS AL SIMMBOLO DEL LEL PASAPORTE}

\begin{tabular}{|c|c|c|c|c|c|c|c|c|c|c|c|}
\hline EXVIRADAS & $C P$ & $C P \mathcal{K}$ & $C \mathcal{V N}$ & cS & $\operatorname{COPN}$ & COER & $\mathrm{co}$ & COES & $\mathscr{F O}$ & FO I & $\mathcal{T U}$ \\
\hline & & $\%$ & $\%$ & $\%$ & $\%$ & & & & & & \\
\hline 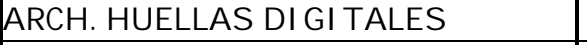 & 25 & $8 \quad 32$ & $3 \quad 38$ & $5 \quad 63$ & $0 \quad 0$ & 0,32 & 3 & 1,00 & 4 & 0,80 & 1,42 \\
\hline BOX REV T TELEFO NICAS & 95 & $49 \quad 52$ & $11 \quad 22$ & $18 \quad 37$ & $20 \quad 41$ & 0,52 & 10 & 1,10 & 5 & 0,28 & 1,78 \\
\hline CAB. CONTTRO L DO CUMEENTOS & 41 & $16 \quad 39$ & $4 \quad 25$ & $7 \quad 44$ & $5 \quad 31$ & 0,39 & 4 & 1,00 & 3 & 0,43 & 1,07 \\
\hline 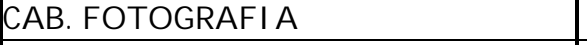 & 36 & $18 \quad 50$ & $5 \quad 28$ & $3 \quad 17$ & $10 \quad 56$ & 0,50 & 6 & 0,83 & 2 & 0,67 & 0,71 \\
\hline 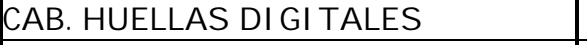 & 30 & 1137 & $3 \quad 27$ & $4 \quad 36$ & $4 \quad 36$ & 0,37 & 4 & 0,75 & 2 & 0,50 & 0,71 \\
\hline CAB. RECEPCION & 76 & $33 \quad 43$ & $8 \quad 24$ & $12 \quad 36$ & $13 \quad 39$ & 0,43 & 7 & 1,14 & 6 & 0,50 & 2,14 \\
\hline $\mathcal{C A g} \mathcal{A}$ & 25 & 1144 & $2 \quad 18$ & $3 \quad 27$ & $6 \quad 55$ & 0,44 & 3 & 0,67 & 1 & 0,33 & 0,36 \\
\hline 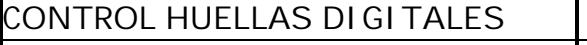 & 70 & $28 \quad 40$ & $5 \quad 18$ & $\begin{array}{ll}11 & 39 \\
\end{array}$ & 1243 & 0,40 & 4 & 1,25 & 5 & 0,45 & 1,78 \\
\hline COORDINADOR & 53 & $25 \quad 47$ & $8 \quad 32$ & $6 \quad 24$ & $11 \quad 44$ & 0,47 & 6 & 1,33 & 0 & 0,00 & 0,00 \\
\hline $\mathcal{D I V} \cdot \mathcal{D A C T}$ I $\perp O S \operatorname{SOPI} \mathcal{A}$ & 31 & $12 \quad 39$ & $\begin{array}{ll}4 & 33 \\
\end{array}$ & $3 \quad 25$ & $5 \quad 42$ & 0,39 & 4 & 1,00 & 4 & 1,33 & 1,42 \\
\hline DIV D DOC. Y CERT. & 117 & 5144 & $15 \quad 29$ & $25 \quad 49$ & $11 \quad 22$ & 0,44 & 12 & 1,25 & 13 & 0,52 & 4,63 \\
\hline DIV I INDICE GENERAL & 39 & $18 \quad 46$ & $5 \quad 28$ & $7 \quad 39$ & $6 \quad 33$ & 0,46 & 5 & 1,00 & 3 & 0,43 & 1,07 \\
\hline 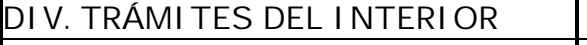 & 60 & $26 \quad 43$ & $3 \quad 12$ & $11 \quad 42$ & 1246 & 0,43 & 5 & 0,60 & 1 & 0,09 & 0,36 \\
\hline 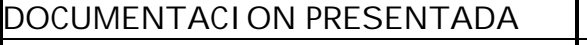 & 240 & 11749 & $15 \quad 13$ & $12 \quad 10$ & $90 \quad 77$ & 0,49 & 17 & 0,88 & 8 & 0,67 & 2,85 \\
\hline $\mathcal{F I C H A} \mathcal{D A C T} I$ I OS COPICA & 24 & 1146 & $3 \quad 27$ & $3 \quad 27$ & $5 \quad 45$ & 0,46 & 4 & 0,75 & 10 & 3,33 & 3,56 \\
\hline FORM. DONACION ORGANOS & 43 & $20 \quad 47$ & $4 \quad 20$ & $4 \quad 20$ & 1260 & 0,47 & 5 & 0,80 & 3 & 0,75 & 1,07 \\
\hline FORM. SO $O$ LICITUTD & 100 & $45 \quad 45$ & $15 \quad 33$ & $10 \quad 22$ & $20 \quad 44$ & 0,45 & 10 & 1,50 & 29 & 2,90 & 10,32 \\
\hline FUINCIONARIO & 90 & $40 \quad 44$ & $9 \quad 23$ & $9 \quad 23$ & $22 \quad 55$ & 0,44 & 6 & 1,50 & 2 & 0,22 & 0,71 \\
\hline 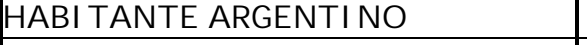 & 34 & $17 \quad 50$ & $3 \quad 18$ & $2 \quad 12$ & $12 \quad 71$ & 0,50 & 3 & 1,00 & 2 & 1,00 & 0,71 \\
\hline INCL. HII O MEENOR 3 AN $\mathcal{A N O S}$ & 72 & $39 \quad 54$ & $7 \quad 18$ & $8 \quad 21$ & $24 \quad 62$ & 0,54 & 8 & 0,88 & 0 & 0,00 & 0,00 \\
\hline LEGAgO & 49 & $20 \quad 41$ & $4 \quad 20$ & $5 \quad 25$ & $11 \quad 55$ & 0,41 & 2 & 2,00 & 6 & 1,20 & 2,14 \\
\hline MEENOR EMAN & 21 & 1048 & $1 \quad 10$ & $1 \quad 10$ & $8 \quad 80$ & 0,48 & 2 & 0,50 & 3 & 3,00 & 1,07 \\
\hline NTRO. CONTTROL & 30 & $12 \quad 40$ & $3 \quad 25$ & $2 \quad 17$ & $7 \quad 58$ & 0,40 & 3 & 1,00 & 3 & 1,50 & 1,07 \\
\hline $\mathbb{N} \mathcal{N R O}$. I D EXN T I FICACION & 33 & $13 \quad 39$ & $3 \quad 23$ & $3 \quad 23$ & $7 \quad 54$ & 0,39 & 3 & 1,00 & 7 & 2,33 & 2,49 \\
\hline OF. OBS ERVADOS & 30 & $16 \quad 53$ & $3 \quad 19$ & $\begin{array}{ll}4 \quad 25 \\
\end{array}$ & 956 & 0,53 & 3 & 1,00 & 3 & 0,75 & 1,07 \\
\hline$O \mathcal{F}$ REVIS ORA & 31 & 1548 & $\begin{array}{ll}4 & 27 \\
\end{array}$ & $6 \quad 40$ & $5 \quad 33$ & 0,48 & 4 & 1,00 & 3 & 0,50 & 1,07 \\
\hline PAS APO RIE & 129 & $53 \quad 41$ & $11 \quad 21$ & $\begin{array}{ll}11 & 21 \\
\end{array}$ & 3158 & 0,41 & 11 & 1,00 & 59 & 5,36 & 21,00 \\
\hline PAS APO RTE $\mathcal{A}$-PSI CADO & 49 & $26 \quad 53$ & $3 \quad 12$ & $5 \quad 19$ & $18 \quad 69$ & 0,53 & 4 & 0,75 & 0 & 0,00 & 0,00 \\
\hline $\mathscr{P A S} \mathcal{A} P O \mathcal{R T} \mathcal{E} O \mathcal{B S}$ ERV $\mathcal{A} \mathcal{A D O}$ & 50 & $34 \quad 68$ & $5 \quad 15$ & $8 \quad 24$ & 2162 & 0,68 & 3 & 1,67 & 1 & 0,13 & 0,36 \\
\hline PAS APO RTE ORI GIN $\mathcal{N A L}$ & 65 & $30 \quad 46$ & $7 \quad 23$ & $10 \quad 33$ & $13 \quad 43$ & 0,46 & 5 & 1,40 & 4 & 0,40 & 1,42 \\
\hline 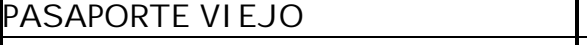 & 50 & $23 \quad 46$ & $\begin{array}{ll}6 \quad 26 \\
\end{array}$ & $\begin{array}{ll}9 & 39 \\
\end{array}$ & $8 \quad 35$ & 0,46 & 4 & 1,50 & 4 & 0,44 & 1,42 \\
\hline $\mathscr{R E} \mathcal{V} \mathcal{A} \perp I \mathcal{D A} \mathscr{P A S} \mathcal{A} \mathcal{P O} R \mathcal{R} \mathcal{I}$ & 128 & $67 \quad 52$ & $9 \quad 13$ & $13 \quad 19$ & $45 \quad 67$ & 0,52 & 10 & 0,90 & 6 & 0,46 & 2,14 \\
\hline S ECTOR EN ETREGA DO CUMMEN N T OS & 53 & $24 \quad 45$ & $\begin{array}{ll}4 \quad 17 \\
\end{array}$ & $10 \quad 42$ & $10 \quad 42$ & 0,45 & 5 & 0,80 & 3 & 0,30 & 1,07 \\
\hline 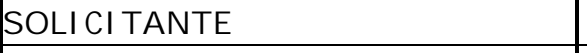 & 119 & 6958 & $17 \quad 25$ & $16 \quad 23$ & $36 \quad 52$ & 0,58 & 15 & 1,13 & 67 & 4,19 & 23,84 \\
\hline $\mathcal{T A L O ́} \mathfrak{X}$ & 50 & $20 \quad 40$ & $3 \quad 15$ & $8 \quad 40$ & $9 \quad 45$ & 0,40 & 4 & 0,75 & 6 & 0,75 & 2,14 \\
\hline 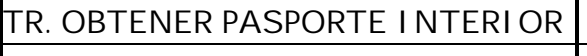 & 40 & 1948 & $3 \quad 16$ & $5 \quad 26$ & 1158 & 0,48 & 3 & 1,00 & 0 & 0,00 & 0,00 \\
\hline VERI FICACION PRONT UUARIO & 23 & 1148 & $2 \quad 18$ & $2 \quad 18$ & $7 \quad 64$ & 0,48 & 2 & 1,00 & 2 & 1,00 & 0,71 \\
\hline
\end{tabular}


MEDICIONES APLICADAS AL LEL "PASAPORTE"

\begin{tabular}{|c|c|c|c|c|c|c|c|}
\hline & \begin{tabular}{|c|} 
CPL \\
2251 \\
\end{tabular} & & & & & & \\
\hline & $C P$ & CPN & $C V N$ & CS & COPN & DSLC & ENTRADAS \\
\hline \multirow{11}{*}{$\begin{array}{l}\stackrel{0}{0} \\
\stackrel{\alpha}{u} \\
\stackrel{\sim}{>}\end{array}$} & 70 & 28 & 5 & 11 & 12 & & CONTROL HUELLAS DIGITALES \\
\hline & 72 & 39 & 7 & 8 & 24 & & INCL. HIJO MENOR 3 AÑOS \\
\hline & 128 & 67 & 9 & 13 & 45 & & REV. PASAPORTE \\
\hline & 40 & 19 & 3 & 5 & 11 & & TR. OBTENER PASPORTE INTERIOR \\
\hline & 23 & 11 & 2 & 2 & 7 & \multirow[b]{2}{*}{5} & VERIFICACION PRONTUARIO \\
\hline & & & & & & & \\
\hline & & 33 & 5 & 8 & 20 & & MEDIA \\
\hline & & 28 & 5 & 8 & 12 & & MEDIANA \\
\hline & & $\# N / A$ & $\# \mathrm{~N} / \mathrm{A}$ & $\# \mathrm{~N} / \mathrm{A}$ & $\# N / A$ & & MODA \\
\hline & & 19 & 3 & 4 & 14 & & DESV ST \\
\hline & & 0.59 & 0.51 & 0.50 & 0,69 & & COEFICIENTE DE VARIACION \\
\hline \multirow{25}{*}{$\begin{array}{l}0 \\
\frac{0}{w} \\
\frac{0}{0} \\
0\end{array}$} & 25 & 8 & 3 & 5 & 0 & & ARCH. HUELLAS DIGITALES \\
\hline & 95 & 49 & 11 & 18 & 20 & & BOX REV. TELEFONICAS \\
\hline & 41 & 16 & 4 & 7 & 5 & & CAB. CONTROL DOCUMENTOS \\
\hline & 36 & 18 & 5 & 3 & 10 & & CAB. FOTOGRAFIA \\
\hline & 30 & 11 & 3 & 4 & 4 & & CAB. HUELLAS DIGITALES \\
\hline & 76 & 33 & 8 & 12 & 13 & & CAB. RECEPCION \\
\hline & 25 & 11 & 2 & 3 & 6 & & CAJA \\
\hline & 31 & 12 & 4 & 3 & 5 & & DIV. DACTILOSCOPIA \\
\hline & 117 & 51 & 15 & 25 & 11 & & DIV. DOC. Y CERT. \\
\hline & 39 & 18 & 5 & 7 & 6 & & DIV. INDICE GENERAL \\
\hline & 60 & 26 & 3 & 11 & 12 & & DIV. TRAMITES DEL INTERIOR \\
\hline & 240 & 117 & 15 & 12 & 90 & & DOCUMENTACION PRESENTADA \\
\hline & 24 & 11 & 3 & 3 & 5 & & FICHA DACTILOSCOPICA \\
\hline & 43 & 20 & 4 & 4 & 12 & & FORM. DONACION ORGANOS \\
\hline & 100 & 45 & 15 & 10 & 20 & & FORM. SOLICITUD \\
\hline & 49 & 20 & 4 & 5 & 11 & & LEGAJO \\
\hline & 30 & 12 & 3 & 2 & 7 & & NRO. CONTROL \\
\hline & 33 & 13 & 3 & 3 & 7 & & NRO. IDENTIFICACION \\
\hline & 30 & 16 & 3 & 4 & 9 & & OF. OBSERVADOS \\
\hline & 31 & 15 & 4 & 6 & 5 & & OF. REVISORA \\
\hline & 129 & 53 & 11 & 11 & 31 & & PASAPORTE \\
\hline & 49 & 26 & 3 & 5 & 18 & & PASAPORTE N-PLICADO \\
\hline & 65 & 30 & 7 & 10 & 13 & & PASAPORTE ORIGINAL \\
\hline & 50 & 23 & 6 & 9 & 8 & & PASAPORTE VIEJO \\
\hline & 53 & 24 & 4 & 10 & 10 & & SECTOR ENTREGA DOCUMENTOS \\
\hline
\end{tabular}




\begin{tabular}{|c|c|c|c|c|c|c|c|}
\hline & $C P$ & CPN & $C V N$ & CS & COPN & DSLC & ENTRADAS \\
\hline & 50 & 20 & 3 & 8 & 9 & & TALON \\
\hline & & & & & & 28 & \\
\hline & & 27 & 6 & 8 & 14 & & MEDIA \\
\hline & & 20 & 4 & 7 & 10 & & MEDIANA \\
\hline & & 11 & 3 & 3 & 5 & & MODA \\
\hline & & 22 & 4 & 5 & 16 & & DESV ST \\
\hline & & 0.80 & 0.66 & 0.63 & 1.17 & & COEFICIENTE DE VARIACION \\
\hline \multirow{11}{*}{ 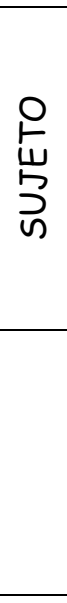 } & 53 & 25 & 8 & 6 & 11 & & COORDINADOR \\
\hline & 90 & 40 & 9 & 9 & 22 & & FUNCIONARIO \\
\hline & 34 & 17 & 3 & 2 & 12 & & HABITANTE ARGENTINO \\
\hline & 21 & 10 & 1 & 1 & 8 & & MENOR EMANCIPADO \\
\hline & 119 & 69 & 17 & 16 & 36 & & SOLICITANTE \\
\hline & & & & & & 5 & \\
\hline & & 32 & 8 & 7 & 18 & & MEDIA \\
\hline & & 25 & 8 & 6 & 12 & & MEDIANA \\
\hline & & $\# N / A$ & $\# \mathrm{~N} / \mathrm{A}$ & $\# \mathrm{~N} / \mathrm{A}$ & $\# N / A$ & & MODA \\
\hline & & 21 & 6 & 5 & 10 & & DESV ST \\
\hline & & ㅇ..6-. & -. .70 & - .7 . & - & $\ldots$ & COEFICIENTE DEVARIACION \\
\hline \multirow{6}{*}{ 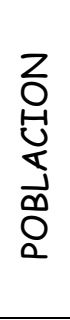 } & & 1057 & 220 & 281 & 556 & 38 & TOTAL \\
\hline & & 29 & 6 & 8 & 15 & & MEDIA \\
\hline & & 20 & 4 & 7 & 11 & & MEDIANA \\
\hline & & 11 & 3 & 3 & 12 & & MODA \\
\hline & & 21 & 4 & 5 & 15 & & DESV ST \\
\hline & & 0.74 & $0,6 \underline{6}$ & 0,62 & 1.03 & & COEFICIENTE DE VARIACION \\
\hline
\end{tabular}


APENDICE G.3

MATRIZ DE ADYACENCIA PONDERADA DEL LEL "PASAPORTE" 


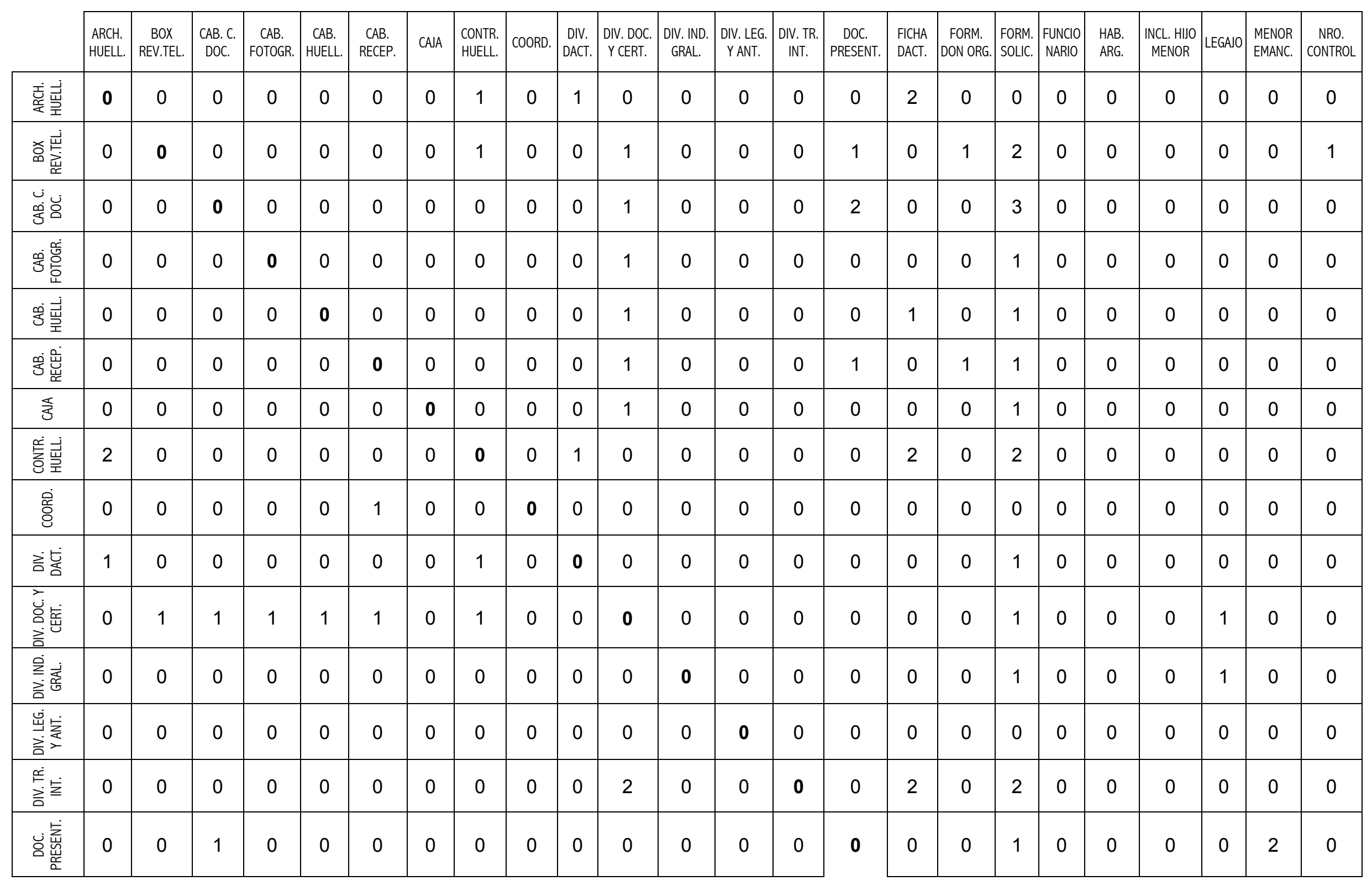




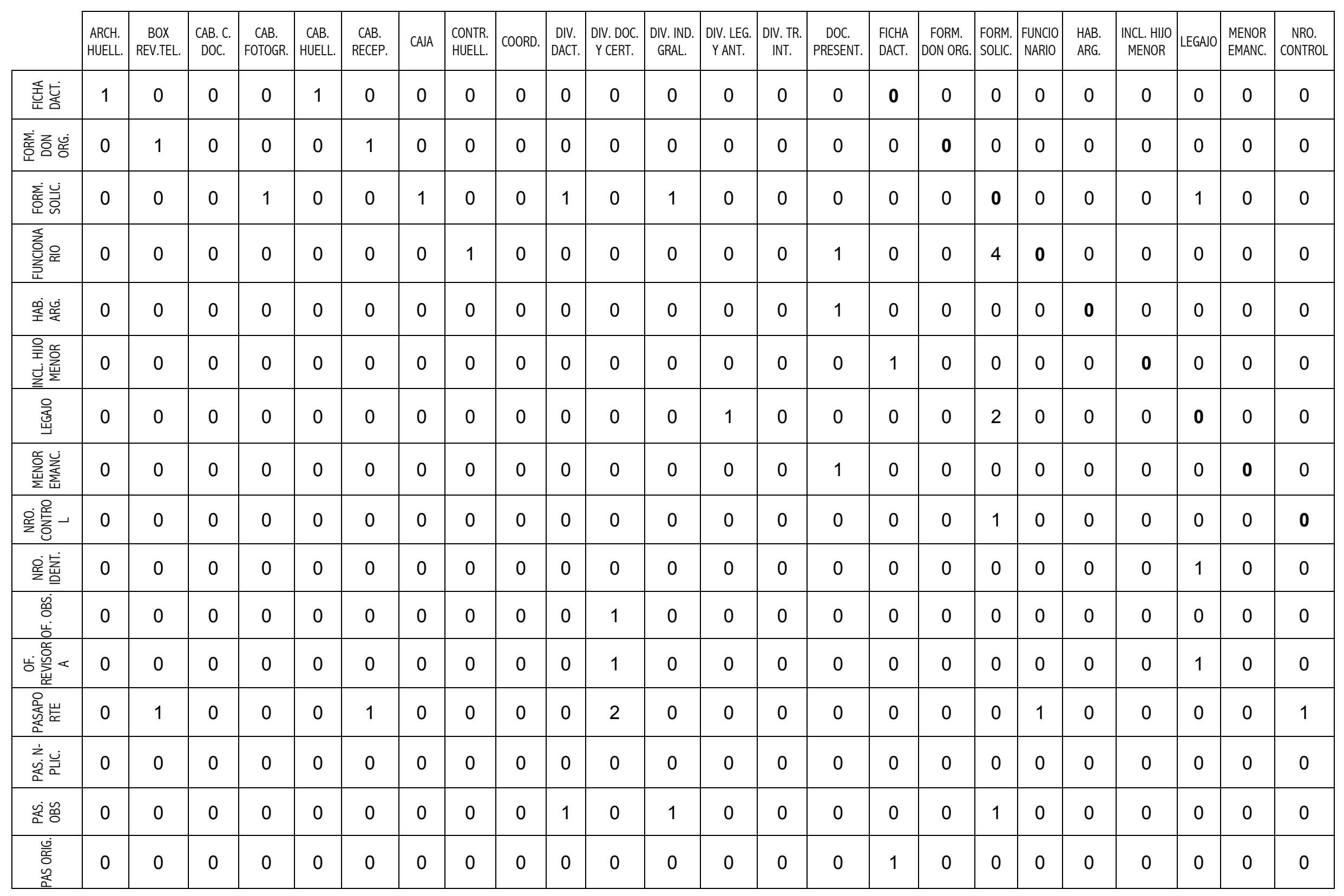




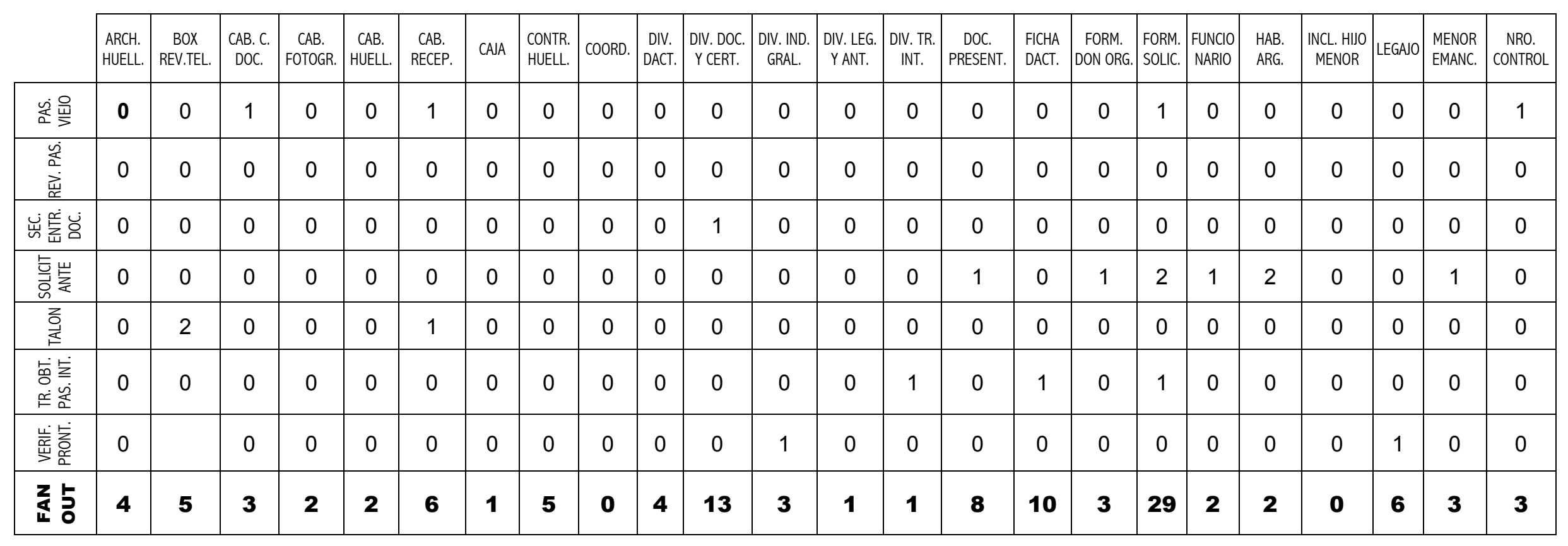




\begin{tabular}{|c|c|c|c|c|c|c|c|c|c|c|c|c|c|c|c|}
\hline & \begin{tabular}{|l} 
NRO. \\
IDENT.
\end{tabular} & $\begin{array}{c}\text { OF. } \\
\text { OBS. }\end{array}$ & \begin{tabular}{|c} 
OF. \\
REVISORA
\end{tabular} & $\begin{array}{l}\text { PASAPO } \\
\text { RTE }\end{array}$ & $\begin{array}{l}\text { PAS. N- } \\
\text { PLIC. }\end{array}$ & $\begin{array}{l}\text { PAS. } \\
\text { OBS }\end{array}$ & $\begin{array}{l}\text { PAS } \\
\text { ORIG. }\end{array}$ & $\begin{array}{l}\text { PAS. } \\
\text { VIEJO }\end{array}$ & $\begin{array}{l}\text { REV. } \\
\text { PAS. }\end{array}$ & $\begin{array}{l}\text { SEC. } \\
\text { ENTR. } \\
\text { DOC. }\end{array}$ & $\begin{array}{c}\text { SOLLITA } \\
\text { NTE }\end{array}$ & TALON & $\begin{array}{l}\text { TR. OBT. } \\
\text { PAS. INT. }\end{array}$ & \begin{tabular}{|l} 
VERIF. \\
PRONT.
\end{tabular} & $\begin{array}{c}\text { FAN } \\
\text { IN }\end{array}$ \\
\hline 要豆 & 0 & 0 & 0 & 0 & 0 & 1 & 0 & 0 & 0 & 0 & 0 & 0 & 0 & 0 & 5 \\
\hline 䧺 & 1 & 0 & 0 & 4 & 0 & 0 & 0 & 0 & 1 & 0 & 3 & 2 & 0 & 0 & 18 \\
\hline 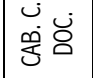 & 0 & 0 & 0 & 1 & 0 & 0 & 0 & 0 & 0 & 0 & 0 & 0 & 0 & 0 & 7 \\
\hline 悉客 & 0 & 0 & 0 & 0 & 0 & 0 & 0 & 0 & 0 & 0 & 1 & 0 & 0 & 0 & 3 \\
\hline 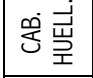 & 0 & 0 & 0 & 0 & 0 & 0 & 0 & 0 & 0 & 0 & 1 & 0 & 0 & 0 & 4 \\
\hline 孚密 & 0 & 0 & 0 & 3 & 0 & 0 & 0 & 0 & 0 & 0 & 4 & 1 & 0 & 0 & 12 \\
\hline$\frac{\pi}{3}$ & 0 & 0 & 0 & 0 & 0 & 0 & 0 & 0 & 0 & 0 & 1 & 0 & 0 & 0 & 3 \\
\hline 㝘志 & 0 & 0 & 0 & 0 & 0 & 0 & 2 & 0 & 1 & 0 & 1 & 0 & 0 & 0 & 11 \\
\hline क्षे & 0 & 0 & 0 & 0 & 0 & 0 & 0 & 0 & 0 & 0 & 5 & 0 & 0 & 0 & 6 \\
\hline 言旁 & 0 & 0 & 0 & 0 & 0 & 0 & 0 & 0 & 0 & 0 & 0 & 0 & 0 & 0 & 3 \\
\hline 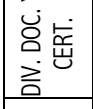 & 0 & 1 & 1 & 5 & 0 & 0 & 0 & 0 & 0 & 1 & 9 & 0 & 0 & 0 & 25 \\
\hline 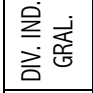 & 1 & 0 & 0 & 0 & 0 & 0 & 0 & 0 & 0 & 0 & 3 & 0 & 0 & 1 & 7 \\
\hline \begin{tabular}{|l} 
岀菨 \\
咅>
\end{tabular} & 0 & 0 & 0 & 0 & 0 & 0 & 0 & 0 & 0 & 0 & 0 & 0 & 0 & 0 & 0 \\
\hline 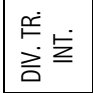 & 0 & 0 & 0 & 2 & 0 & 0 & 1 & 1 & 0 & 0 & 1 & 0 & 0 & 0 & 11 \\
\hline 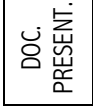 & 0 & 0 & 0 & 1 & 0 & 0 & 1 & 2 & 1 & 0 & 3 & 0 & 0 & 0 & 12 \\
\hline
\end{tabular}




\begin{tabular}{|c|c|c|c|c|c|c|c|c|c|c|c|c|c|c|c|}
\hline & $\begin{array}{l}\text { NRO. } \\
\text { IDENT. }\end{array}$ & $\begin{array}{c}\text { OF. } \\
\text { OBS. }\end{array}$ & \begin{tabular}{|c} 
OF. \\
REVIISORA
\end{tabular} & $\begin{array}{l}\text { PASAPO } \\
\text { RTE }\end{array}$ & $\begin{array}{l}\text { PAS. N- } \\
\text { PLIC. }\end{array}$ & $\begin{array}{l}\text { PAS. } \\
\text { OBS }\end{array}$ & $\begin{array}{l}\text { PAS } \\
\text { ORIG. }\end{array}$ & $\begin{array}{l}\text { PAS. } \\
\text { VIEJO }\end{array}$ & $\begin{array}{l}\text { REV. } \\
\text { PAS. }\end{array}$ & $\begin{array}{l}\text { SEC. } \\
\text { ENTR. } \\
\text { DOC. }\end{array}$ & $\begin{array}{l}\text { SOLICITA } \\
\text { NTE }\end{array}$ & TALON & \begin{tabular}{|l} 
TR. OBT. \\
PAS. INT.
\end{tabular} & \begin{tabular}{|l} 
VERIF. \\
PRONT.
\end{tabular} & $\begin{array}{c}\text { FAN } \\
\text { IN }\end{array}$ \\
\hline 薩总 & 0 & 0 & 0 & 0 & 0 & 0 & 0 & 0 & 0 & 0 & 1 & 0 & 0 & 0 & 3 \\
\hline 要 & 0 & 0 & 0 & 1 & 0 & 0 & 0 & 0 & 0 & 0 & 1 & 0 & 0 & 0 & 4 \\
\hline 产总 & 0 & 0 & 0 & 2 & 0 & 0 & 0 & 0 & 0 & 0 & 3 & 0 & 0 & 0 & 10 \\
\hline 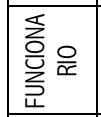 & 0 & 0 & 0 & 2 & 0 & 0 & 0 & 0 & 0 & 0 & 0 & 0 & 0 & 1 & 9 \\
\hline 呈脢 & 0 & 0 & 0 & 0 & 0 & 0 & 0 & 0 & 0 & 0 & 1 & 0 & 0 & 0 & 2 \\
\hline 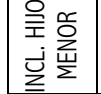 & 1 & 0 & 0 & 2 & 0 & 0 & 0 & 0 & 0 & 0 & 4 & 0 & 0 & 0 & 8 \\
\hline 趂 & 1 & 0 & 0 & 1 & 0 & 0 & 0 & 0 & 0 & 0 & 0 & 0 & 0 & 0 & 5 \\
\hline 窟竞 & 0 & 0 & 0 & 0 & 0 & 0 & 0 & 0 & 0 & 0 & 0 & 0 & 0 & 0 & 1 \\
\hline 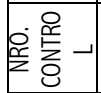 & 0 & 0 & 0 & 0 & 0 & 0 & 0 & 0 & 1 & 0 & 0 & 0 & 0 & 0 & 2 \\
\hline 实怠 & 0 & 0 & 0 & 2 & 0 & 0 & 0 & 0 & 0 & 0 & 0 & 0 & 0 & 0 & 3 \\
\hline 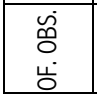 & 0 & 0 & 0 & 1 & 0 & 0 & 0 & 0 & 0 & 0 & 2 & 0 & 0 & 0 & 4 \\
\hline 竞 & 0 & 0 & 0 & 1 & 0 & 0 & 0 & 0 & 0 & 0 & 3 & 0 & 0 & 0 & 6 \\
\hline 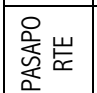 & 1 & 0 & 0 & 0 & 0 & 0 & 0 & 0 & 1 & 1 & 2 & 0 & 0 & 0 & 11 \\
\hline 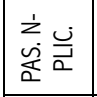 & 0 & 0 & 0 & 3 & 0 & 0 & 0 & 1 & 0 & 0 & 1 & 0 & 0 & 0 & 5 \\
\hline 容 嵓 & 0 & 1 & 1 & 1 & 0 & 0 & 0 & 0 & 0 & 0 & 2 & 0 & 0 & 0 & 8 \\
\hline 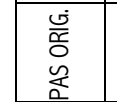 & 2 & 0 & 0 & 3 & 0 & 0 & 0 & 0 & 0 & 0 & 4 & 0 & 0 & 0 & 10 \\
\hline
\end{tabular}




\begin{tabular}{|c|c|c|c|c|c|c|c|c|c|c|c|c|c|c|c|}
\hline & \begin{tabular}{|l} 
NRO. \\
IDENT.
\end{tabular} & $\begin{array}{l}\text { OF. } \\
\text { OBS. }\end{array}$ & \begin{tabular}{|c} 
OF. \\
REVISORA
\end{tabular} & $\begin{array}{c}\text { PASAPO } \\
\text { RTE }\end{array}$ & $\begin{array}{l}\text { PAS. N- } \\
\text { PLIC. }\end{array}$ & $\begin{array}{l}\text { PAS. } \\
\text { OBS }\end{array}$ & $\begin{array}{c}\text { PAS } \\
\text { ORIG. }\end{array}$ & $\begin{array}{l}\text { PAS. } \\
\text { VIEJO }\end{array}$ & $\begin{array}{l}\text { REV. } \\
\text { PAS. }\end{array}$ & $\begin{array}{l}\text { SEC. } \\
\text { ENTR. } \\
\text { DOC. }\end{array}$ & $\begin{array}{c}\text { SOLLITA } \\
\text { NTE }\end{array}$ & TALON & \begin{tabular}{|l} 
TR. OBT. \\
PAS. INT.
\end{tabular} & \begin{tabular}{|l} 
VERIF. \\
PRONT.
\end{tabular} & $\begin{array}{c}\text { FAN } \\
\text { IN }\end{array}$ \\
\hline 嚆㥯 & 0 & 0 & 0 & 3 & 0 & 0 & 0 & 0 & 0 & 0 & 2 & 0 & 0 & 0 & 9 \\
\hline 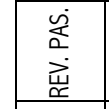 & 0 & 0 & 0 & 11 & 0 & 0 & 0 & 0 & 0 & 0 & 2 & 0 & 0 & 0 & 13 \\
\hline 离密号 & 0 & 1 & 1 & 2 & 0 & 0 & 0 & 0 & 0 & 0 & 4 & 1 & 0 & 0 & 10 \\
\hline 竞峞 & 0 & 0 & 0 & 5 & 0 & 0 & 0 & 0 & 1 & 0 & 0 & 2 & 0 & 0 & 16 \\
\hline 䃕 & 0 & 0 & 0 & 2 & 0 & 0 & 0 & 0 & 0 & 1 & 2 & 0 & 0 & 0 & 8 \\
\hline 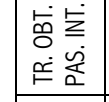 & 0 & 0 & 0 & 1 & 0 & 0 & 0 & 0 & 0 & 0 & 1 & 0 & 0 & 0 & 5 \\
\hline 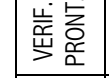 & 0 & 0 & 0 & 0 & 0 & 0 & 0 & 0 & 0 & 0 & 0 & 0 & 0 & 0 & 2 \\
\hline $\begin{array}{ll}2 & 5 \\
4 & 5\end{array}$ & 7 & 3 & 3 & 59 & 0 & 1 & 4 & 4 & 6 & 3 & 67 & 6 & 0 & 2 & \\
\hline
\end{tabular}


APENDICE H.1

TABLA DE MEDICIONES APLICADAS AL SIMBOLO DEL LEL
"LEL \& ESCENARIOS"

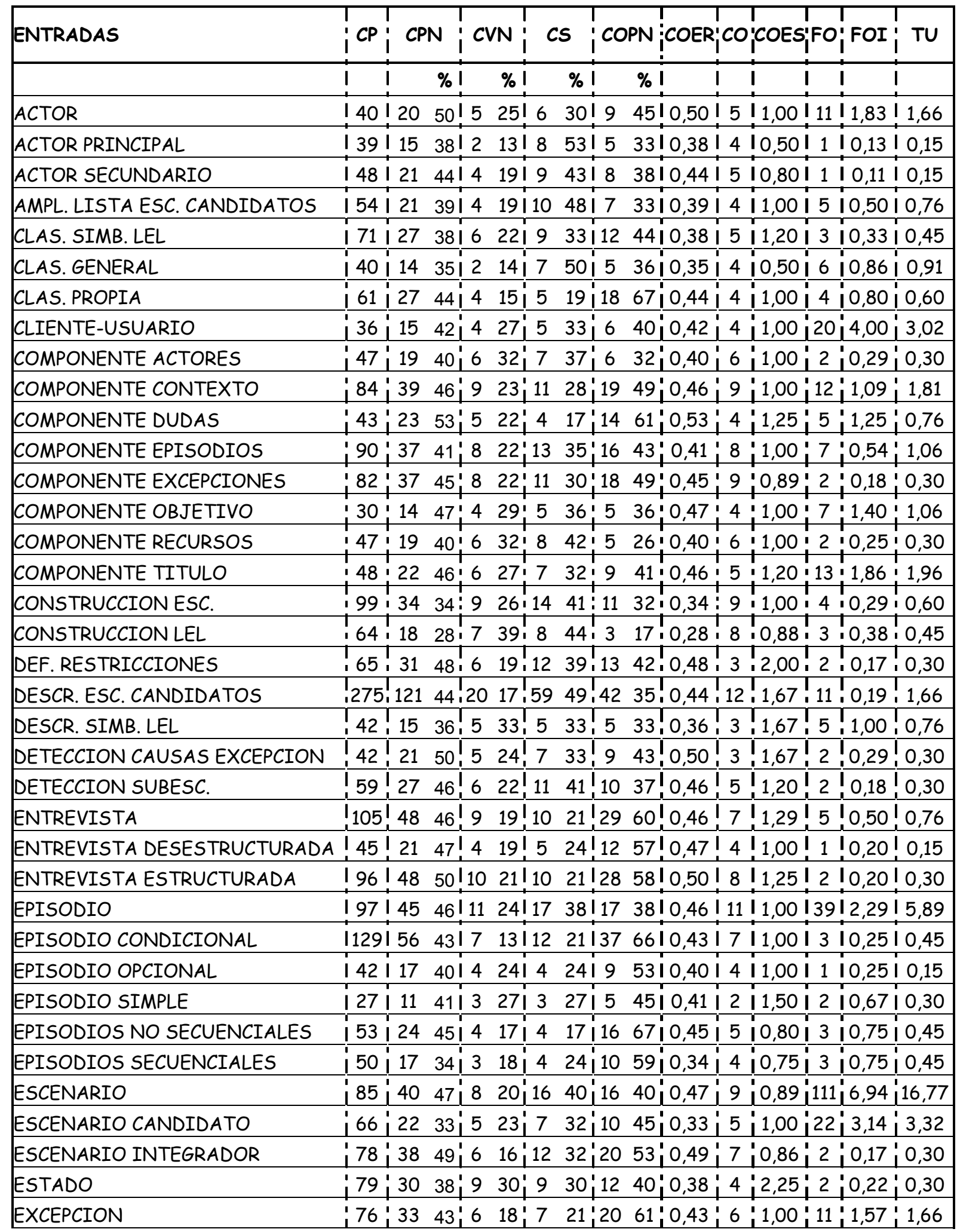




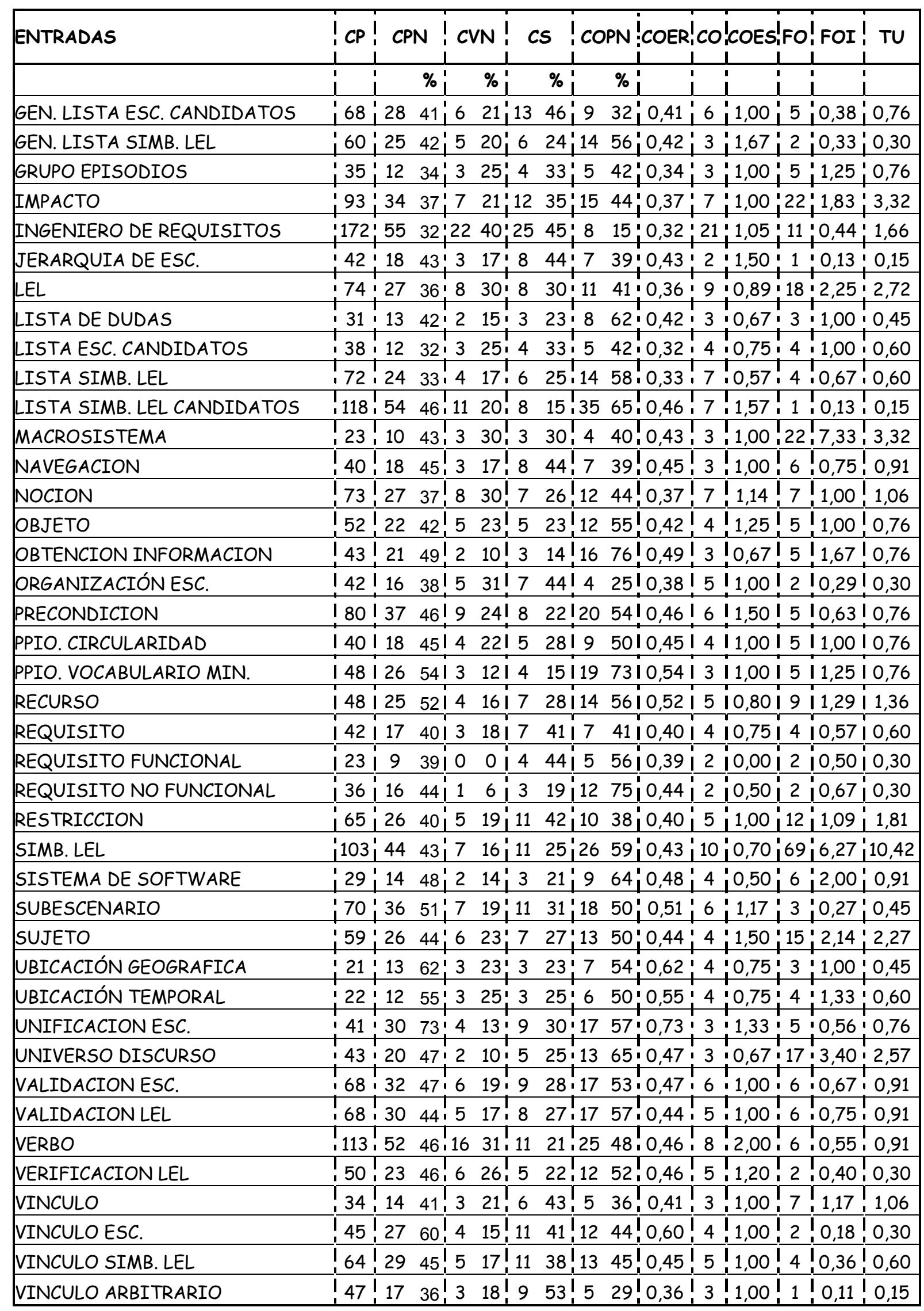


APENDICE H.2

TABLA DE MEDICIONES APLICADAS AL LEL "LEL \& ESCENARIOS"

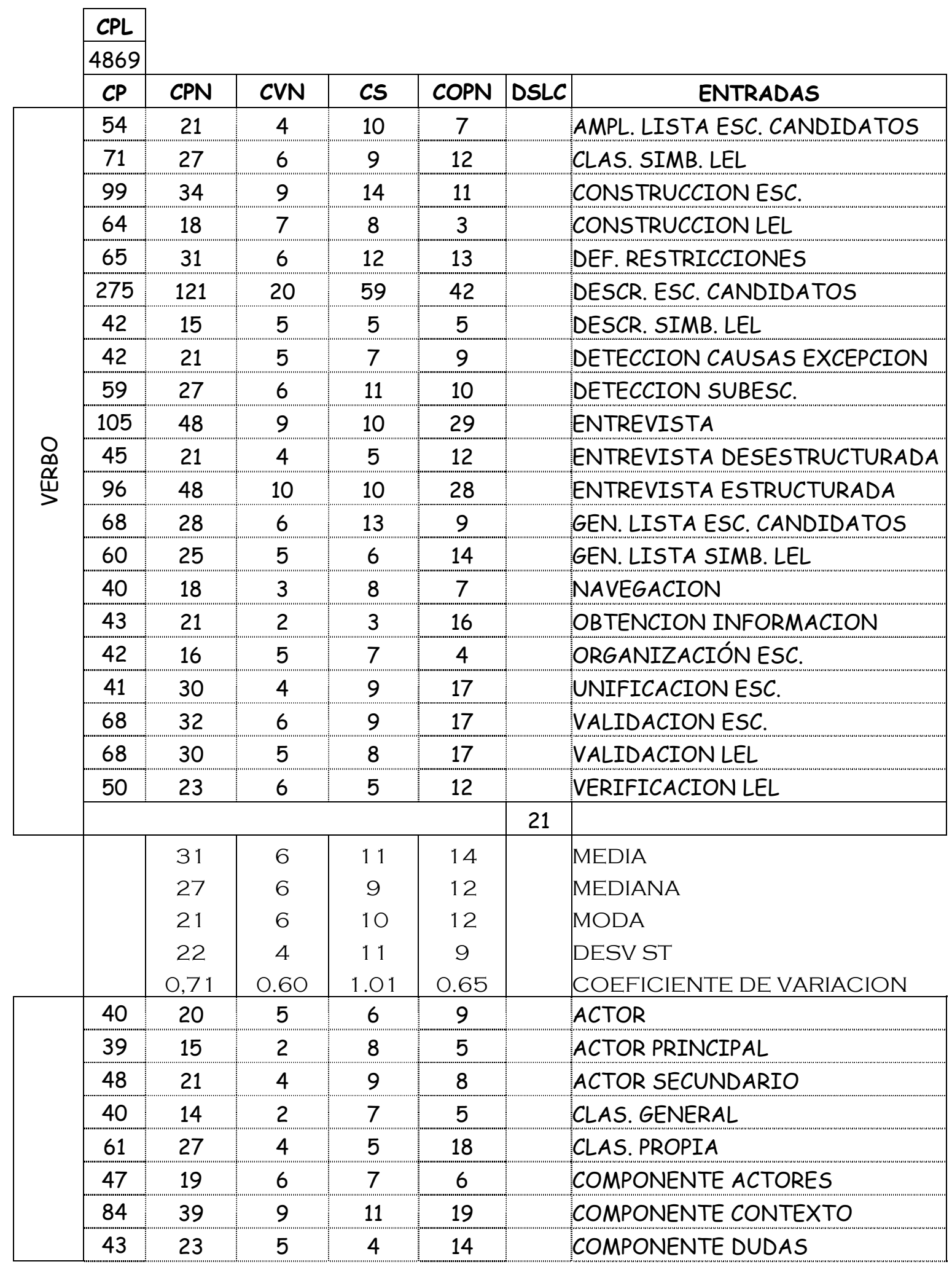




\begin{tabular}{|c|c|c|c|c|c|c|}
\hline$C P$ & CPN & $C V N$ & CS & COPN & DSLC & ENTRADAS \\
\hline 90 & 37 & 8 & 13 & 16 & & COMP. EPISODIOS \\
\hline 82 & 37 & 8 & 11 & 18 & & COMP. EXCEPCIONES \\
\hline 30 & 14 & 4 & 5 & 5 & & COMP. OBJETIVO \\
\hline 47 & 19 & 6 & 8 & 5 & & COMP. RECURSOS \\
\hline 48 & 22 & 6 & 7 & 9 & & COMP. TÍTULO \\
\hline 97 & 45 & 11 & 17 & 17 & & EPISODIO \\
\hline 129 & 56 & 7 & 12 & 37 & & EPISODIO CONDICIONAL \\
\hline 42 & 17 & 4 & 4 & 9 & & EPISODIO OPCIONAL \\
\hline 27 & 11 & 3 & 3 & 5 & & EPISODIO SIMPLE \\
\hline 53 & 24 & 4 & 4 & 16 & & EPISODIOS NO SECUENCIALES \\
\hline 50 & 17 & 3 & 4 & 10 & & EPISODIOS SECUENCIALES \\
\hline 85 & 40 & 8 & 16 & 16 & & ESCENARIO \\
\hline 66 & 22 & 5 & 7 & 10 & & ESC. CANDIDATO \\
\hline 78 & 38 & 6 & 12 & 20 & & ESC. INTEGRADOR \\
\hline 79 & 30 & 9 & 6 & 15 & & ESTADO \\
\hline 76 & 33 & 6 & 7 & 20 & & EXCEPCION \\
\hline 35 & 12 & 3 & 4 & 5 & & GRUPO EPISODIOS \\
\hline 93 & 34 & 7 & 12 & 15 & & IMPACTO \\
\hline 42 & 18 & 3 & 8 & 7 & & JERARQUIA ESCENARIOS \\
\hline 74 & 27 & 8 & 8 & 11 & & LEL \\
\hline 31 & 13 & 2 & 3 & 8 & & LISTA DUDAS \\
\hline 38 & 12 & 3 & 4 & 5 & & LISTA ESC. CANDIDATOS \\
\hline 72 & 24 & 4 & 6 & 14 & & LISTA SIMB. LEL \\
\hline 118 & 54 & 11 & 8 & 35 & & LISTA SIMB. LEL CANDIDATOS \\
\hline 23 & 10 & 3 & 3 & 4 & & MACROSISTEMA \\
\hline 73 & 27 & 8 & 7 & 12 & & NOCION \\
\hline 52 & 22 & 5 & 5 & 12 & & OBJETO \\
\hline 80 & 37 & 9 & 8 & 20 & & PRECONDICION \\
\hline 40 & 18 & 4 & 5 & 9 & & PPIO. CIRCULARIDAD \\
\hline 48 & 26 & 3 & 4 & 19 & & PPIO. VOCABULARIO MIN. \\
\hline 48 & 25 & 4 & 7 & 14 & & RECURSO \\
\hline 42 & 17 & 3 & 7 & 7 & & REQUISITO \\
\hline 23 & 9 & 0 & 4 & 5 & & REQUISITO FUNCIONAL \\
\hline 36 & 16 & 1 & 3 & 12 & & REQUISITO NO FUNCIONAL \\
\hline 65 & 26 & 5 & 11 & 10 & & RESTRICCION \\
\hline 103 & 44 & 7 & 11 & 26 & & SIMB. LEL \\
\hline 29 & 14 & 2 & 3 & 9 & & SISTEMA SOFTWARE \\
\hline 70 & 36 & 7 & 11 & 18 & & SUBESCENARIO \\
\hline 59 & 26 & 6 & 7 & 13 & & SUJETO \\
\hline 21 & 13 & 3 & 3 & 7 & & UBICACIÓN GEOGRAFICA \\
\hline 22 & 12 & 3 & 3 & 6 & & UBICACIÓN TEMPORAL \\
\hline 43 & 20 & 2 & 5 & 13 & & UNIVERSO DISCURSO \\
\hline 113 & 52 & 16 & 11 & 25 & & VERBO \\
\hline 34 & 14 & 3 & 6 & 5 & & VINCULO \\
\hline
\end{tabular}




\begin{tabular}{|c|c|c|c|c|c|c|c|}
\hline & $C P$ & CPN & CVN & CS & COPN & DSLC & ENTRADAS \\
\hline & 45 & 27 & 4 & 11 & 12 & \multicolumn{2}{|r|}{ VINCULO ESC. } \\
\hline & 64 & 29 & 5 & 11 & 13 & & VINCULO SIMB. LEL \\
\hline & 47 & 17 & 3 & 9 & 5 & & VINCULO ARBITRARIO \\
\hline & & & & & & \multicolumn{2}{|l|}{55} \\
\hline & & 25 & 5 & 7 & 13 & & MEDIA \\
\hline & & 22 & 4 & 7 & 12 & & MEDIANA \\
\hline & & 14 & 3 & 7 & 5 & & MODA \\
\hline & & 12 & 3 & 3 & 7 & & DESV ST \\
\hline & & 0.46 & 0.57 & 0.49 & 0,59 & & COEFICIENTE DE VARIACION \\
\hline \multirow{3}{*}{$\begin{array}{l}\text { 은 } \\
\text { 岁 } \\
\text { 勺 }\end{array}$} & 36 & 15 & 4 & 5 & 6 & & CLIENTE-USUARIO \\
\hline & 172 & 55 & 22 & 25 & 8 & & INGENIERO REQUISITOS \\
\hline & & & & & & 2 & \\
\hline \multirow{11}{*}{ 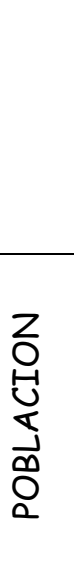 } & & 35 & 13 & 15 & 7 & & MEDIA \\
\hline & & 35 & 13 & 15 & 7 & & MEDIANA \\
\hline & & $\# N / A$ & $\# N / A$ & $\# \mathrm{~N} / \mathrm{A}$ & $\# N / A$ & & MODA \\
\hline & & 20 & 9 & 10 & 1 & & DESV ST \\
\hline & & 0.57. & - 0.69. & 0.67 & _. & & COEFICIENTE DE VARIACION \\
\hline & 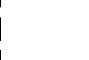 & 2096 & 441 & 662 & 996 & 78 & TOTAL \\
\hline & & 27 & 6 & 8 & 13 & & MEDIA \\
\hline & & 23,5 & 5 & 7 & 12 & & MEDIANA \\
\hline & 1 & 27 & 4 & 7 & 5 & & MODA \\
\hline & 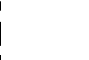 & 16 & 4 & 7 & 8 & & DESV ST \\
\hline & 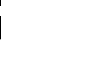 & 0,61 & - $\underline{0}, \underline{6} \underline{0}$. & $0,86$. & _. & & COEFICIENTE DEVARIACION \\
\hline
\end{tabular}


APENDICE H.3

MATRIZ DE ADYACENCIA PONDERADA DEL LEL "LEL \& ESCENARIOS" 


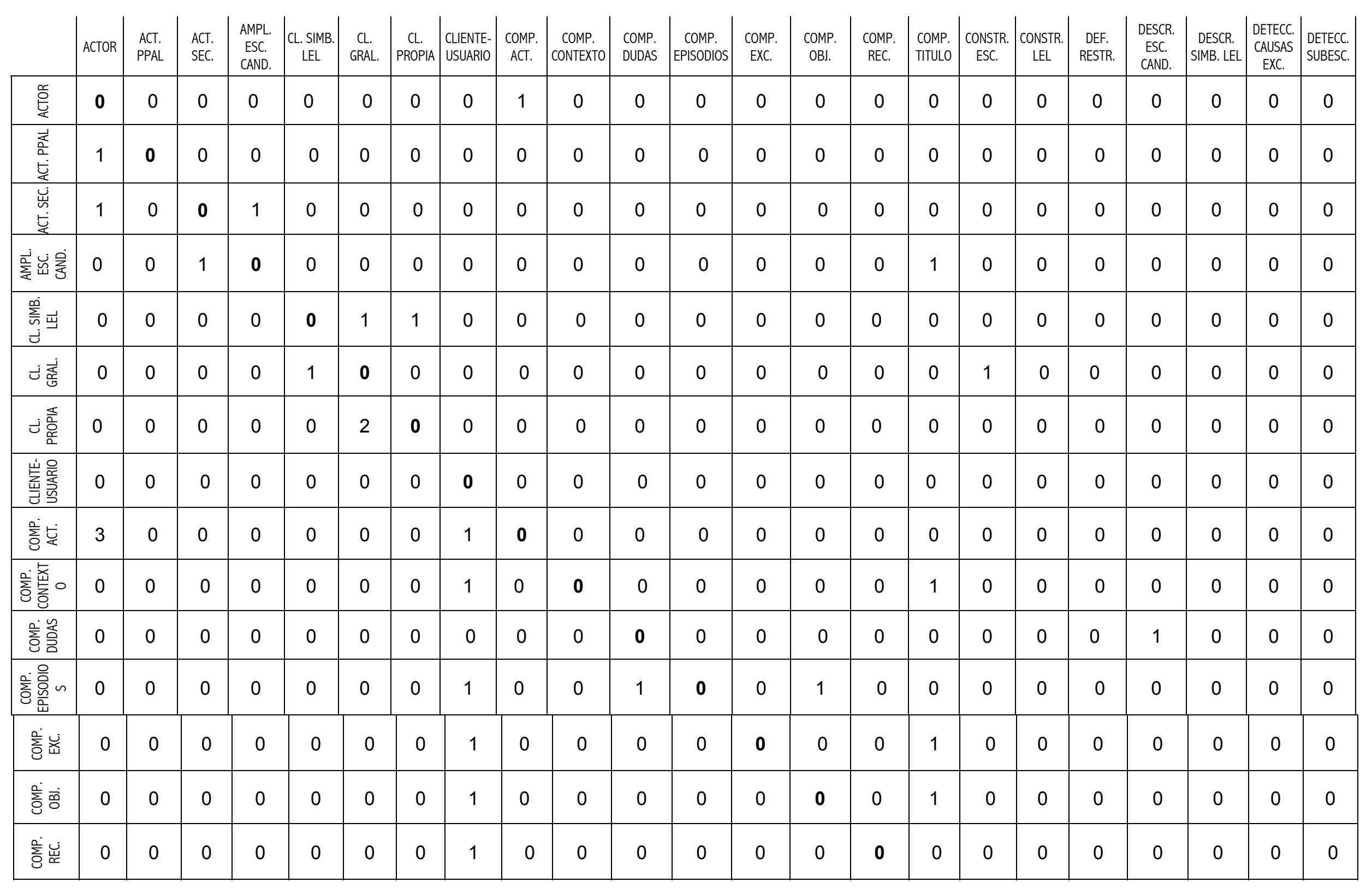




\begin{tabular}{|c|c|c|c|c|c|c|c|c|c|c|c|c|c|c|c|c|c|c|c|c|c|c|c|}
\hline & ACTOR & $\begin{array}{l}\text { ACT. } \\
\text { PPAL }\end{array}$ & $\begin{array}{l}\text { ACT. } \\
\text { SEC. }\end{array}$ & $\begin{array}{l}\text { AMPL. } \\
\text { ESC. } \\
\text { CAND. }\end{array}$ & $\begin{array}{c}\text { CL. SIMB. } \\
\text { LEL }\end{array}$ & CL. GRAL. & $\begin{array}{c}\text { CL. } \\
\text { PROPIA }\end{array}$ & $\begin{array}{l}\text { CLIENTE- } \\
\text { USUARIO }\end{array}$ & $\begin{array}{l}\text { COMP. } \\
\text { ACT. }\end{array}$ & $\begin{array}{c}\text { COMP. } \\
\text { CONTEXTO }\end{array}$ & $\begin{array}{l}\text { COMP. } \\
\text { DUDAS }\end{array}$ & \begin{tabular}{|} 
COMP. \\
EPISODIO \\
S
\end{tabular} & $\begin{array}{c}\text { COMP. } \\
\text { EXC. }\end{array}$ & $\begin{array}{c}\text { COMP. } \\
\text { OBJ. }\end{array}$ & $\begin{array}{l}\text { COMP. } \\
\text { REC. }\end{array}$ & $\begin{array}{l}\text { COMP. } \\
\text { TITULO }\end{array}$ & $\begin{array}{l}\text { CONSTR. } \\
\text { ESC. }\end{array}$ & $\begin{array}{c}\text { CONSTR. } \\
\text { LEL }\end{array}$ & $\begin{array}{c}\text { DEF. } \\
\text { RESTR. }\end{array}$ & $\begin{array}{l}\text { DESCR. } \\
\text { ESC. } \\
\text { CAND. }\end{array}$ & $\begin{array}{c}\text { DESCR. } \\
\text { SIMB. LEL }\end{array}$ & \begin{tabular}{|c|} 
DETECC. \\
CAUSAS \\
EXC.
\end{tabular} & $\begin{array}{l}\text { DETECC. } \\
\text { SUBESC. }\end{array}$ \\
\hline 品号点 & 0 & 0 & 0 & 0 & 0 & 0 & 0 & 1 & 0 & 0 & 0 & 0 & 0 & 0 & 0 & 0 & 0 & 0 & 0 & 0 & 0 & 0 & 0 \\
\hline 喜 & 0 & 0 & 0 & 1 & 0 & 0 & 0 & 0 & 0 & 0 & 0 & 0 & 0 & 0 & 0 & 0 & 0 & 0 & 0 & 1 & 0 & 0 & 0 \\
\hline 竞山 & 0 & 0 & 0 & 0 & 1 & 0 & 0 & 0 & 0 & 0 & 0 & 0 & 0 & 0 & 0 & 0 & 0 & 0 & 0 & 0 & 1 & 0 & 0 \\
\hline 㞻怠 & 0 & 0 & 0 & 0 & 0 & 0 & 0 & 0 & 0 & 0 & 0 & 0 & 0 & 0 & 0 & 0 & 0 & 0 & 0 & 0 & 0 & 0 & 0 \\
\hline 总总㤩 & 2 & 0 & 0 & 0 & 0 & 0 & 0 & 0 & 0 & 3 & 1 & 0 & 1 & 3 & 0 & 1 & 0 & 0 & 1 & 0 & 0 & 1 & 0 \\
\hline 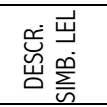 & 0 & 0 & 0 & 0 & 0 & 0 & 0 & 0 & 0 & 0 & 0 & 0 & 0 & 0 & 0 & 0 & 0 & 0 & 0 & 0 & 0 & 0 & 0 \\
\hline 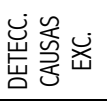 & 0 & 0 & 0 & 0 & 0 & 0 & 0 & 0 & 0 & 1 & 0 & 0 & 0 & 0 & 0 & 0 & 0 & 0 & 0 & 0 & 0 & 0 & 0 \\
\hline 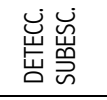 & 0 & 0 & 0 & 0 & 0 & 0 & 0 & 0 & 0 & 0 & 0 & 0 & 0 & 0 & 0 & 1 & 0 & 0 & 0 & 0 & 0 & 0 & 0 \\
\hline 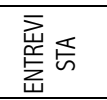 & 0 & 0 & 0 & 0 & 0 & 0 & 0 & 2 & 0 & 0 & 0 & 0 & 0 & 0 & 0 & 0 & 0 & 1 & 0 & 0 & 0 & 0 & 0 \\
\hline 苫苫岀 & 0 & 0 & 0 & 0 & 0 & 0 & 0 & 2 & 0 & 0 & 0 & 0 & 0 & 0 & 0 & 0 & 0 & 0 & 0 & 0 & 0 & 0 & 0 \\
\hline 菨怠 & 0 & 0 & 0 & 0 & 0 & 0 & 0 & 1 & 0 & 0 & 1 & 0 & 0 & 0 & 0 & 0 & 0 & 0 & 0 & 1 & 1 & 0 & 0 \\
\hline 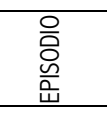 & 2 & 0 & 0 & 0 & 0 & 0 & 0 & 0 & 0 & 1 & 0 & 1 & 0 & 1 & 0 & 0 & 0 & 0 & 0 & 0 & 0 & 0 & 0 \\
\hline 芒 & 0 & 0 & 0 & 0 & 0 & 0 & 0 & 0 & 0 & 0 & 0 & 1 & 0 & 0 & 0 & 0 & 0 & 0 & 0 & 1 & 0 & 0 & 0 \\
\hline 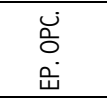 & 0 & 0 & 0 & 0 & 0 & 0 & 0 & 0 & 0 & 0 & 0 & 1 & 0 & 0 & 0 & 0 & 0 & 0 & 0 & 1 & 0 & 0 & 0 \\
\hline 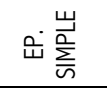 & 0 & 0 & 0 & 0 & 0 & 0 & 0 & 0 & 0 & 0 & 0 & 1 & 0 & 0 & 0 & 0 & 0 & 0 & 0 & 1 & 0 & 0 & 0 \\
\hline 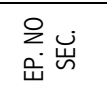 & 0 & 0 & 0 & 0 & 0 & 0 & 0 & 0 & 0 & 0 & 0 & 1 & 0 & 0 & 0 & 0 & 0 & 0 & 0 & 1 & 0 & 0 & 0 \\
\hline
\end{tabular}




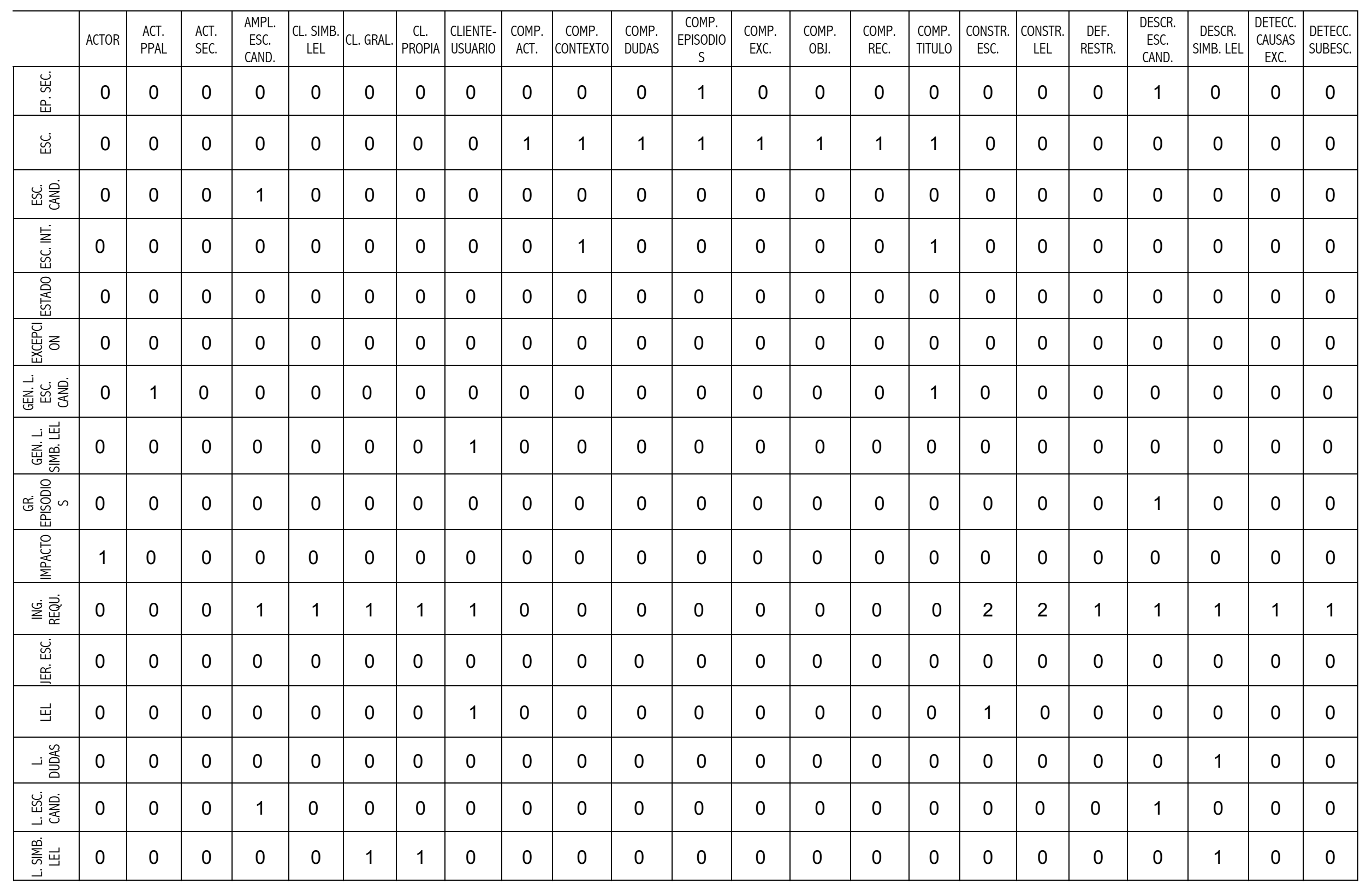




\begin{tabular}{|c|c|c|c|c|c|c|c|c|c|c|c|c|c|c|c|c|c|c|c|c|c|c|c|}
\hline & ACTOR & $\begin{array}{l}\text { ACT. } \\
\text { PPAL } \\
\end{array}$ & $\begin{array}{l}\text { ACT. } \\
\text { SEC. }\end{array}$ & $\begin{array}{c}\text { AMPL. } \\
\text { ESC. } \\
\text { CAND. }\end{array}$ & \begin{tabular}{|c} 
CL. SIMB \\
LEL
\end{tabular} & CL. GRAL. & \begin{tabular}{|c} 
CL. \\
PROPIA \\
\end{tabular} & \begin{tabular}{|l|} 
CLIENTE- \\
USUARIO
\end{tabular} & $\begin{array}{l}\text { COMP. } \\
\text { ACT. }\end{array}$ & \begin{tabular}{|c} 
COMP. \\
CONTEXTO \\
\end{tabular} & $\begin{array}{l}\text { COMP. } \\
\text { DUDAS } \\
\end{array}$ & $\mid \begin{array}{c}\text { COMP. } \\
\text { EPISODIO } \\
S\end{array}$ & $\begin{array}{c}\text { COMP. } \\
\text { EXC. }\end{array}$ & $\begin{array}{c}\text { COMP. } \\
\text { OBJ. }\end{array}$ & $\begin{array}{c}\text { COMP. } \\
\text { REC. }\end{array}$ & \begin{tabular}{|c|} 
COMP. \\
TITULO \\
\end{tabular} & \begin{tabular}{|c|} 
CONSTR. \\
ESC. \\
\end{tabular} & \begin{tabular}{|c|} 
CONSTR. \\
LEL \\
\end{tabular} & $\begin{array}{c}\text { DEF. } \\
\text { RESTR. }\end{array}$ & $\begin{array}{c}\text { DESCR. } \\
\text { ESC. } \\
\text { CAND. }\end{array}$ & \begin{tabular}{|c} 
DESCR. \\
SIMB. LEL \\
\end{tabular} & \begin{tabular}{|l|} 
DETECC. \\
CAUSAS \\
EXC. \\
\end{tabular} & \begin{tabular}{|l} 
DETECC. \\
SUBESC. \\
\end{tabular} \\
\hline$\sum_{\bar{v}}^{\infty} \overrightarrow{\sum_{j}}$ & 0 & 0 & 0 & 0 & 0 & 0 & 0 & 1 & 0 & 0 & 0 & 0 & 0 & 0 & 0 & 0 & 0 & 0 & 0 & 0 & 0 & 0 & 0 \\
\hline 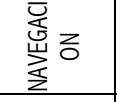 & 0 & 0 & 0 & 0 & 0 & 0 & 0 & 0 & 0 & 0 & 0 & 0 & 0 & 0 & 0 & 0 & 0 & 0 & 0 & 0 & 0 & 0 & 0 \\
\hline 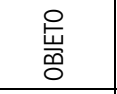 & 0 & 0 & 0 & 0 & 0 & 0 & 0 & 0 & 0 & 0 & 0 & 0 & 0 & 0 & 0 & 0 & 0 & 0 & 0 & 0 & 0 & 0 & 0 \\
\hline 宮崖 & 0 & 0 & 0 & 0 & 0 & 0 & 0 & 0 & 0 & 0 & 0 & 0 & 0 & 0 & 0 & 0 & 0 & 0 & 0 & 0 & 0 & 0 & 0 \\
\hline 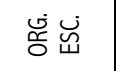 & 0 & 0 & 0 & 0 & 0 & 0 & 0 & 0 & 0 & 0 & 0 & 0 & 0 & 0 & 0 & 0 & 0 & 0 & 0 & 0 & 0 & 0 & 1 \\
\hline 总 & 0 & 0 & 0 & 0 & 0 & 0 & 0 & 0 & 0 & 1 & 0 & 0 & 0 & 0 & 0 & 0 & 0 & 0 & 0 & 0 & 0 & 0 & 0 \\
\hline 㞯。 & 0 & 0 & 0 & 0 & 0 & 0 & 0 & 0 & 0 & 0 & 0 & 0 & 0 & 0 & 1 & 0 & 0 & 0 & 0 & 0 & 0 & 0 & 0 \\
\hline 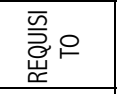 & 0 & 0 & 0 & 0 & 0 & 0 & 0 & 1 & 0 & 0 & 0 & 0 & 0 & 0 & 0 & 0 & 0 & 0 & 0 & 0 & 0 & 0 & 0 \\
\hline 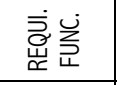 & 0 & 0 & 0 & 0 & 0 & 0 & 0 & 0 & 0 & 0 & 0 & 0 & 0 & 0 & 0 & 0 & 0 & 0 & 0 & 0 & 0 & 0 & 0 \\
\hline 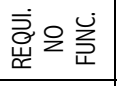 & 0 & 0 & 0 & 0 & 0 & 0 & 0 & 0 & 0 & 0 & 0 & 0 & 0 & 0 & 0 & 0 & 0 & 0 & 0 & 0 & 0 & 0 & 0 \\
\hline 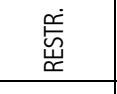 & 0 & 0 & 0 & 0 & 0 & 0 & 0 & 0 & 0 & 1 & 0 & 0 & 0 & 0 & 0 & 0 & 0 & 0 & 0 & 0 & 0 & 0 & 0 \\
\hline 离 & 0 & 0 & 0 & 0 & 0 & 1 & 1 & 0 & 0 & 0 & 0 & 0 & 0 & 0 & 0 & 0 & 0 & 0 & 0 & 0 & 0 & 0 & 0 \\
\hline 吻营 & 0 & 0 & 0 & 0 & 0 & 0 & 0 & 0 & 0 & 0 & 0 & 0 & 0 & 0 & 0 & 0 & 0 & 0 & 0 & 0 & 0 & 0 & 0 \\
\hline
\end{tabular}




\begin{tabular}{|c|c|c|c|c|c|c|c|c|c|c|c|c|c|c|c|c|c|c|c|c|c|c|c|}
\hline & ACTOR & $\begin{array}{l}\text { ACT. } \\
\text { PPAL } \\
\end{array}$ & $\begin{array}{l}\text { ACT. } \\
\text { SEC. }\end{array}$ & $\begin{array}{c}\text { AMPL. } \\
\text { ESC. } \\
\text { CAND. }\end{array}$ & \begin{tabular}{|c}
$\mid \begin{array}{c}\text { CL. SIMB } \\
\text { LEL }\end{array}$ \\
\end{tabular} & CL. GRAL. & $\begin{array}{c}\text { CL. } \\
\text { PROPIA } \\
\end{array}$ & $\begin{array}{l}\text { CLIENTE- } \\
\text { USUARIO } \\
\end{array}$ & $\begin{array}{l}\text { COMP. } \\
\text { ACT. }\end{array}$ & \begin{tabular}{|c|} 
COMP. \\
CONTEXTO
\end{tabular} & $\begin{array}{l}\text { COMP. } \\
\text { DUDAS }\end{array}$ & $\begin{array}{c}\text { COMP. } \\
\text { EPISODIO } \\
\text { S } \\
\end{array}$ & $\begin{array}{c}\text { COMP. } \\
\text { EXC. }\end{array}$ & $\begin{array}{c}\text { COMP. } \\
\text { OBJ. }\end{array}$ & $\begin{array}{c}\text { COMP. } \\
\text { REC. }\end{array}$ & $\begin{array}{l}\text { COMP. } \\
\text { TITULO } \\
\end{array}$ & $\begin{array}{c}\text { CONSTR. } \\
\text { ESC. }\end{array}$ & $\begin{array}{c}\text { CONSTR. } \\
\text { LEL } \\
\end{array}$ & $\begin{array}{c}\text { DEF. } \\
\text { RESTR. }\end{array}$ & $\begin{array}{c}\text { DESCR. } \\
\text { ESC. } \\
\text { CAND. }\end{array}$ & $\begin{array}{c}\text { DESCR. } \\
\text { SIMB. LEL } \\
\end{array}$ & \begin{tabular}{|c|} 
DETECC. \\
CAUSAS \\
EXC. \\
\end{tabular} & $\begin{array}{l}\text { DEEECC. } \\
\text { SUBESC. }\end{array}$ \\
\hline $\begin{array}{l}\text { 岕 } \\
\text { 岕 }\end{array}$ & 1 & 0 & 0 & 0 & 0 & 0 & 0 & 0 & 0 & 0 & 0 & 0 & 0 & 0 & 0 & 2 & 0 & 0 & 0 & 0 & 0 & 0 & 0 \\
\hline 乌 & 0 & 0 & 0 & 0 & 0 & 0 & 0 & 0 & 0 & 1 & 0 & 0 & 0 & 0 & 0 & 0 & 0 & 0 & 0 & 0 & 0 & 0 & 0 \\
\hline 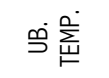 & 0 & 0 & 0 & 0 & 0 & 0 & 0 & 0 & 0 & 1 & 0 & 0 & 0 & 0 & 0 & 0 & 0 & 0 & 0 & 0 & 0 & 0 & 0 \\
\hline 윅 & 0 & 0 & 0 & 0 & 0 & 0 & 0 & 0 & 0 & 0 & 0 & 0 & 0 & 0 & 0 & 0 & 0 & 0 & 0 & 0 & 0 & 0 & 0 \\
\hline$\dot{~}$ & 0 & 0 & 0 & 0 & 0 & 0 & 0 & 1 & 0 & 0 & 1 & 0 & 0 & 0 & 0 & 0 & 0 & 0 & 0 & 0 & 0 & 0 & 0 \\
\hline$\dot{s} \vec{s}$ & 0 & 0 & 0 & 0 & 0 & 0 & 0 & 2 & 0 & 0 & 0 & 0 & 0 & 0 & 0 & 0 & 0 & 0 & 0 & 0 & 0 & 0 & 0 \\
\hline 岁 & 0 & 0 & 0 & 0 & 0 & 0 & 0 & 0 & 0 & 0 & 0 & 0 & 0 & 0 & 0 & 2 & 0 & 0 & 0 & 0 & 0 & 0 & 0 \\
\hline 岁离出 & 0 & 0 & 0 & 0 & 0 & 0 & 0 & 0 & 0 & 0 & 0 & 0 & 0 & 0 & 0 & 0 & 0 & 0 & 0 & 0 & 0 & 0 & 0 \\
\hline 引选 & 0 & 0 & 0 & 0 & 0 & 0 & 0 & 0 & 0 & 0 & 0 & 0 & 0 & 0 & 0 & 0 & 0 & 0 & 0 & 0 & 0 & 0 & 0 \\
\hline 25 & 11 & 1 & 1 & 5 & 3 & 6 & 4 & 20 & 2 & 12 & 5 & 7 & 2 & 7 & 2 & 13 & 4 & 3 & 2 & 11 & 5 & 2 & 2 \\
\hline
\end{tabular}




\begin{tabular}{|c|c|c|c|c|c|c|c|c|c|c|c|c|c|c|c|c|c|c|c|c|c|c|c|c|}
\hline & $\begin{array}{c}\text { ENTREVIST } \\
\text { A }\end{array}$ & $\begin{array}{c}\text { ENTR. } \\
\text { DESESTR. }\end{array}$ & $\begin{array}{l}\text { ENTR. } \\
\text { ESTR. }\end{array}$ & EPISODIO & $\begin{array}{c}\text { EP. } \\
\text { COND. }\end{array}$ & EP. OPC. & $\begin{array}{c}\text { EP. } \\
\text { SIMPLE }\end{array}$ & $\begin{array}{l}\text { EP. NO } \\
\text { SEC. }\end{array}$ & EP. SEC. & ESC. & $\begin{array}{l}\text { ESC. } \\
\text { CAND. }\end{array}$ & $\begin{array}{l}\text { ESC. } \\
\text { INT. }\end{array}$ & ESTADO & EXCEPCION & \begin{tabular}{|c} 
GEN. L. \\
ESC. \\
CAND.
\end{tabular} & $\begin{array}{c}\text { GEN. L. } \\
\text { SIMB. } \\
\text { LEL }\end{array}$ & $\begin{array}{c}\text { GR. } \\
\text { EPISODIOS }\end{array}$ & IMPACTO & $\begin{array}{c}\text { ING. } \\
\text { REQU. }\end{array}$ & JER. ESC. & LEL & $\begin{array}{c}\text { L. } \\
\text { DLDAS }\end{array}$ & $\begin{array}{l}\text { L. ESC. } \\
\text { CAND. }\end{array}$ & $\begin{array}{l}\text { L. SIMB } \\
\text { LEL }\end{array}$ \\
\hline 产 & 0 & 0 & 0 & 2 & 0 & 0 & 0 & 0 & 0 & 1 & 0 & 0 & 0 & 0 & 0 & 0 & 0 & 0 & 0 & 0 & 0 & 0 & 0 & 0 \\
\hline 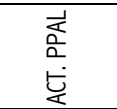 & 0 & 0 & 0 & 0 & 0 & 0 & 0 & 0 & 0 & 0 & 0 & 0 & 0 & 0 & 1 & 0 & 0 & 1 & 0 & 0 & 1 & 0 & 0 & 0 \\
\hline 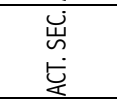 & 0 & 0 & 0 & 0 & 0 & 0 & 0 & 0 & 0 & 0 & 0 & 0 & 0 & 0 & 0 & 0 & 0 & 1 & 0 & 0 & 1 & 0 & 0 & 0 \\
\hline 定出完 & 0 & 0 & 0 & 0 & 0 & 0 & 0 & 0 & 0 & 0 & 4 & 0 & 0 & 0 & 0 & 0 & 0 & 2 & 0 & 0 & 1 & 0 & 1 & 0 \\
\hline 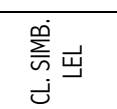 & 0 & 0 & 0 & 0 & 0 & 0 & 0 & 0 & 0 & 0 & 0 & 0 & 0 & 0 & 0 & 0 & 0 & 1 & 0 & 0 & 0 & 0 & 0 & 2 \\
\hline 己定 & 0 & 0 & 0 & 0 & 0 & 0 & 0 & 0 & 0 & 0 & 0 & 0 & 1 & 0 & 0 & 0 & 0 & 0 & 0 & 0 & 0 & 0 & 0 & 0 \\
\hline 己高 & 0 & 0 & 0 & 0 & 0 & 0 & 0 & 0 & 0 & 0 & 0 & 0 & 0 & 0 & 0 & 0 & 0 & 0 & 0 & 0 & 0 & 0 & 0 & 0 \\
\hline 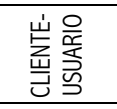 & 1 & 0 & 0 & 0 & 0 & 0 & 0 & 0 & 0 & 0 & 0 & 0 & 0 & 0 & 0 & 0 & 0 & 0 & 1 & 0 & 0 & 0 & 0 & 0 \\
\hline 竞安 & 0 & 0 & 0 & 1 & 0 & 0 & 0 & 0 & 0 & 2 & 0 & 0 & 0 & 0 & 0 & 0 & 0 & 0 & 0 & 0 & 0 & 0 & 0 & 0 \\
\hline 这。 & 0 & 0 & 0 & 0 & 0 & 0 & 0 & 0 & 0 & 5 & 0 & 1 & 0 & 0 & 0 & 0 & 0 & 0 & 0 & 0 & 0 & 0 & 0 & 0 \\
\hline 产言 & 0 & 0 & 0 & 0 & 0 & 0 & 0 & 0 & 0 & 3 & 0 & 0 & 0 & 0 & 0 & 0 & 0 & 0 & 0 & 0 & 0 & 0 & 0 & 0 \\
\hline 言咅旁 & 0 & 0 & 0 & 4 & 0 & 0 & 0 & 1 & 1 & 3 & 0 & 0 & 0 & 0 & 0 & 0 & 1 & 0 & 0 & 0 & 0 & 0 & 0 & 0 \\
\hline 这岑 & 0 & 0 & 0 & 0 & 0 & 0 & 0 & 0 & 0 & 5 & 0 & 0 & 0 & 4 & 0 & 0 & 0 & 0 & 0 & 0 & 0 & 0 & 0 & 0 \\
\hline 运审 & 0 & 0 & 0 & 0 & 0 & 0 & 0 & 0 & 0 & 3 & 0 & 0 & 0 & 0 & 0 & 0 & 0 & 0 & 0 & 0 & 0 & 0 & 0 & 0 \\
\hline$\sum_{0}^{0}$ & 0 & 0 & 0 & 1 & 0 & 0 & 0 & 0 & 0 & 3 & 0 & 0 & 0 & 0 & 0 & 0 & 0 & 0 & 0 & 0 & 0 & 0 & 0 & 0 \\
\hline
\end{tabular}




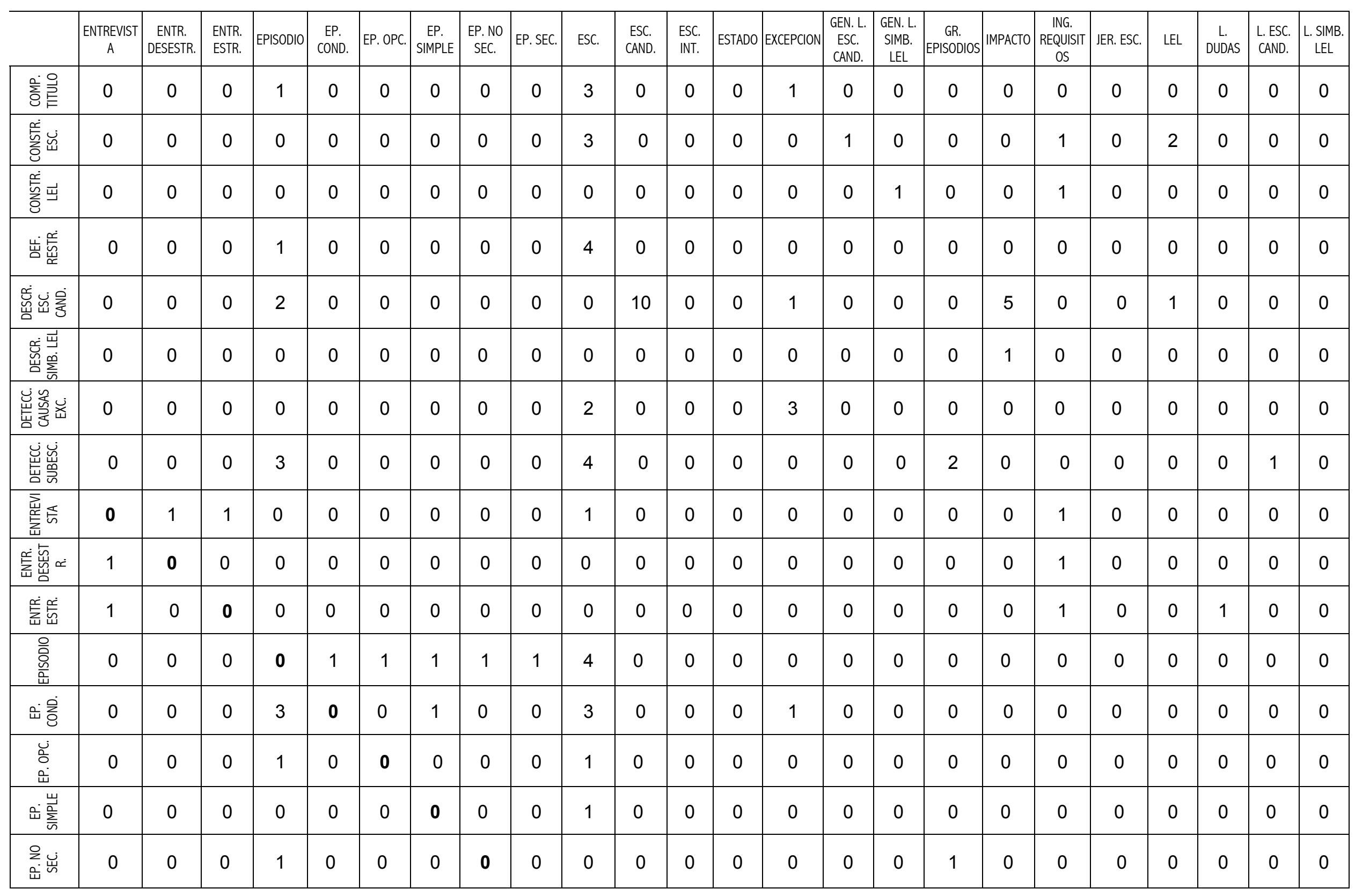




\begin{tabular}{|c|c|c|c|c|c|c|c|c|c|c|c|c|c|c|c|c|c|c|c|c|c|c|c|c|}
\hline & $\begin{array}{c}\text { ENTREVIST } \\
\text { A }\end{array}$ & $\begin{array}{c}\text { ENTR. } \\
\text { DESESTR. }\end{array}$ & $\begin{array}{l}\text { ENTR. } \\
\text { ESTR. }\end{array}$ & EPISODIO & $\begin{array}{c}\text { EP. } \\
\text { COND. }\end{array}$ & EP. OPC. & $\begin{array}{c}\text { EP. } \\
\text { SIMPLE } \\
\end{array}$ & \begin{tabular}{|l} 
EP. NO \\
SEC.
\end{tabular} & EP. SEC. & ESC. & $\begin{array}{l}\text { ESC. } \\
\text { CAND. }\end{array}$ & $\begin{array}{l}\text { ESC. } \\
\text { INT. }\end{array}$ & ESTADO & EXCEPCION & \begin{tabular}{|c|} 
GEN. L. \\
ESC. \\
CAND. \\
\end{tabular} & \begin{tabular}{|c|} 
GEN. L. \\
SIMB. \\
LEL \\
\end{tabular} & \begin{tabular}{|c|} 
GR. \\
EPISODIOS \\
\end{tabular} & IMPACTO & $\begin{array}{c}\text { ING. } \\
\text { REQU. } \\
\end{array}$ & JER. ESC. & LEL & \begin{tabular}{|c} 
L. \\
DUDAS \\
\end{tabular} & $\begin{array}{l}\text { L. ESC. } \\
\text { CAND. }\end{array}$ & $\begin{array}{c}\text { L. SIMB. } \\
\text { LEL }\end{array}$ \\
\hline 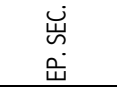 & 0 & 0 & 0 & 1 & 0 & 0 & 0 & 0 & 0 & 0 & 0 & 0 & 0 & 0 & 0 & 0 & 1 & 0 & 0 & 0 & 0 & 0 & 0 & 0 \\
\hline 岁 & 0 & 0 & 0 & 0 & 0 & 0 & 0 & 0 & 0 & 0 & 0 & 0 & 0 & 0 & 0 & 0 & 0 & 0 & 0 & 0 & 1 & 0 & 0 & 0 \\
\hline 岀悎 & 0 & 0 & 0 & 0 & 0 & 0 & 0 & 0 & 0 & 1 & 0 & 0 & 0 & 0 & 1 & 0 & 0 & 0 & 0 & 0 & 0 & 0 & 1 & 0 \\
\hline 岀完 & 0 & 0 & 0 & 1 & 0 & 0 & 0 & 0 & 0 & 7 & 0 & 0 & 0 & 0 & 0 & 0 & 0 & 0 & 0 & 1 & 0 & 0 & 0 & 0 \\
\hline 是 & 0 & 0 & 0 & 0 & 0 & 0 & 0 & 0 & 0 & 0 & 0 & 0 & 0 & 0 & 0 & 0 & 0 & 1 & 0 & 0 & 0 & 0 & 0 & 0 \\
\hline $\begin{array}{l}\text { 을 } \\
\text { 嘊 } \\
\end{array}$ & 0 & 0 & 0 & 1 & 1 & 0 & 0 & 0 & 0 & 5 & 0 & 0 & 0 & 0 & 0 & 0 & 0 & 0 & 0 & 0 & 0 & 0 & 0 & 0 \\
\hline 怠出察 & 0 & 0 & 0 & 0 & 0 & 0 & 0 & 0 & 0 & 0 & 4 & 0 & 0 & 0 & 0 & 0 & 0 & 3 & 0 & 0 & 2 & 0 & 1 & 0 \\
\hline 离悹空 & 0 & 0 & 0 & 0 & 0 & 0 & 0 & 0 & 0 & 0 & 0 & 0 & 0 & 0 & 0 & 0 & 0 & 0 & 0 & 0 & 1 & 0 & 0 & 0 \\
\hline 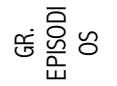 & 0 & 0 & 0 & 1 & 0 & 0 & 0 & 1 & 1 & 0 & 0 & 0 & 0 & 0 & 0 & 0 & 0 & 0 & 0 & 0 & 0 & 0 & 0 & 0 \\
\hline 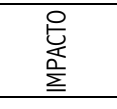 & 0 & 0 & 0 & 0 & 0 & 0 & 0 & 0 & 0 & 0 & 1 & 0 & 0 & 0 & 0 & 0 & 0 & 0 & 0 & 0 & 0 & 0 & 0 & 0 \\
\hline 崖 & 1 & 0 & 0 & 0 & 0 & 0 & 0 & 0 & 0 & 1 & 0 & 0 & 0 & 0 & 1 & 1 & 0 & 0 & 0 & 0 & 0 & 0 & 0 & 0 \\
\hline 密总 & 0 & 0 & 0 & 2 & 0 & 0 & 0 & 0 & 0 & 3 & 0 & 1 & 0 & 0 & 0 & 0 & 0 & 0 & 0 & 0 & 0 & 0 & 0 & 0 \\
\hline Ш & 0 & 0 & 0 & 0 & 0 & 0 & 0 & 0 & 0 & 1 & 0 & 0 & 0 & 0 & 0 & 0 & 0 & 0 & 0 & 0 & 0 & 0 & 0 & 0 \\
\hline- 䇾 & 0 & 0 & 0 & 0 & 0 & 0 & 0 & 0 & 0 & 0 & 0 & 0 & 0 & 0 & 0 & 0 & 0 & 0 & 0 & 0 & 0 & 0 & 0 & 0 \\
\hline 岂豈 & 0 & 0 & 0 & 0 & 0 & 0 & 0 & 0 & 0 & 1 & 0 & 0 & 0 & 0 & 1 & 0 & 0 & 0 & 0 & 0 & 0 & 0 & 0 & 0 \\
\hline$\sum_{j}^{\infty}$ & 0 & 0 & 0 & 0 & 0 & 0 & 0 & 0 & 0 & 0 & 0 & 0 & 0 & 0 & 0 & 0 & 0 & 0 & 0 & 0 & 0 & 0 & 0 & 0 \\
\hline
\end{tabular}




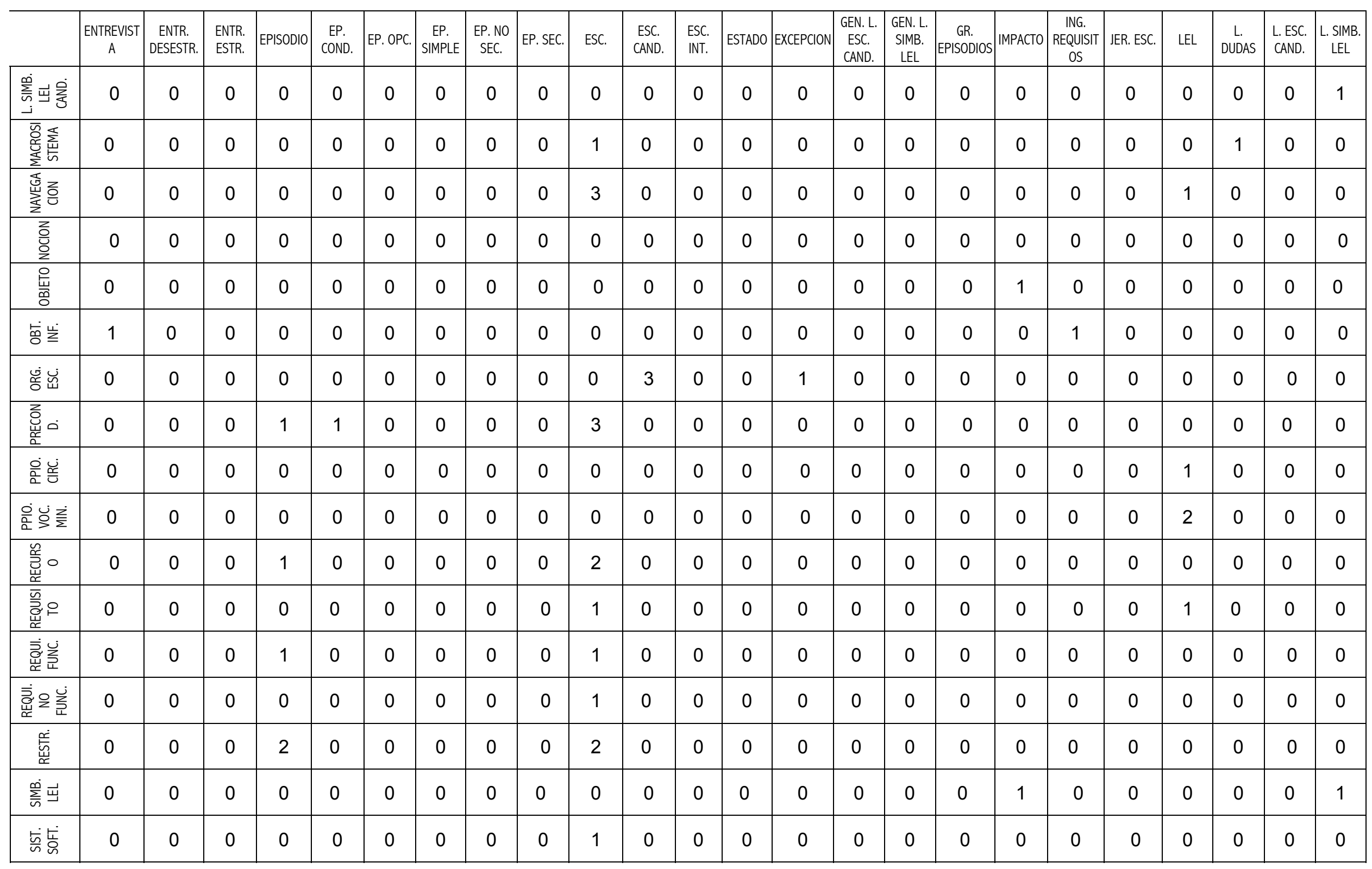




\begin{tabular}{|c|c|c|c|c|c|c|c|c|c|c|c|c|c|c|c|c|c|c|c|c|c|c|c|c|}
\hline & $\begin{array}{c}\text { ENTREVIST } \\
\text { A }\end{array}$ & $\begin{array}{c}\text { ENTR. } \\
\text { DESESTR. }\end{array}$ & $\begin{array}{l}\text { ENTR. } \\
\text { ESTR. }\end{array}$ & EPISODIO & $\begin{array}{c}\text { EP. } \\
\text { COND. } \\
\end{array}$ & EP. OPC. & $\begin{array}{c}\text { EP. } \\
\text { SIMPLE } \\
\end{array}$ & $\begin{array}{c}\text { EP. NO } \\
\text { SEC. }\end{array}$ & EP. SEC. & ESC. & $\begin{array}{l}\text { ESC. } \\
\text { CAND. }\end{array}$ & $\begin{array}{l}\text { ESC. } \\
\text { INT. }\end{array}$ & ESTADO & EXCEPCION & $\begin{array}{r}\text { GEN. L. } \\
\text { ESC. } \\
\text { CAND. } \\
\end{array}$ & \begin{tabular}{|c|} 
GEN. L. \\
SIMB. \\
LEL \\
\end{tabular} & \begin{tabular}{|c|} 
GR. \\
EPISODIOS \\
\end{tabular} & IMPACTO & \begin{tabular}{|c|} 
INGG \\
REQUISIT \\
OS \\
\end{tabular} & JER. ESC. & LEL & $\begin{array}{c}\text { L. } \\
\text { DUDAS } \\
\end{array}$ & $\begin{array}{l}\text { L. ESC. } \\
\text { CAND. } \\
\end{array}$ & $\begin{array}{c}\text { L. SIMB. } \\
\text { LEL }\end{array}$ \\
\hline $\begin{array}{l}\text { 岀 } \\
\text { 鲁 }\end{array}$ & 0 & 0 & 0 & 3 & 0 & 0 & 0 & 0 & 0 & 4 & 0 & 0 & 0 & 0 & 0 & 0 & 0 & 0 & 0 & 0 & 0 & 0 & 0 & 0 \\
\hline 惫 & 0 & 0 & 0 & 0 & 0 & 0 & 0 & 0 & 0 & 0 & 0 & 0 & 0 & 0 & 0 & 0 & 0 & 1 & 0 & 0 & 0 & 0 & 0 & 0 \\
\hline פو & 0 & 0 & 0 & 0 & 0 & 0 & 0 & 0 & 0 & 1 & 0 & 0 & 0 & 0 & 0 & 0 & 0 & 0 & 0 & 0 & 0 & 0 & 0 & 0 \\
\hline 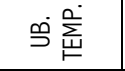 & 0 & 0 & 0 & 0 & 0 & 0 & 0 & 0 & 0 & 1 & 0 & 0 & 0 & 0 & 0 & 0 & 0 & 0 & 0 & 0 & 0 & 0 & 0 & 0 \\
\hline 訔出 & 0 & 0 & 0 & 2 & 0 & 0 & 0 & 0 & 0 & 5 & 0 & 0 & 0 & 0 & 0 & 0 & 0 & 0 & 0 & 0 & 0 & 0 & 0 & 0 \\
\hline 을 & 0 & 0 & 0 & 0 & 0 & 0 & 0 & 0 & 0 & 0 & 0 & 0 & 0 & 0 & 0 & 0 & 0 & 0 & 1 & 0 & 0 & 0 & 0 & 0 \\
\hline 宾出 & 0 & 0 & 0 & 1 & 0 & 0 & 0 & 0 & 0 & 5 & 0 & 0 & 0 & 0 & 0 & 0 & 0 & 0 & 1 & 0 & 0 & 0 & 0 & 0 \\
\hline$\dot{\vec{s}} \overrightarrow{\mathrm{s}}$ & 0 & 0 & 1 & 0 & 0 & 0 & 0 & 0 & 0 & 0 & 0 & 0 & 0 & 0 & 0 & 0 & 0 & 0 & 1 & 0 & 0 & 1 & 0 & 0 \\
\hline 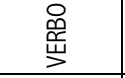 & 0 & 0 & 0 & 0 & 0 & 0 & 0 & 0 & 0 & 0 & 0 & 0 & 1 & 0 & 0 & 0 & 0 & 4 & 0 & 0 & 0 & 0 & 0 & 0 \\
\hline 辤出 & 0 & 0 & 0 & 0 & 0 & 0 & 0 & 0 & 0 & 0 & 0 & 0 & 0 & 0 & 0 & 0 & 0 & 0 & 0 & 0 & 1 & 0 & 0 & 0 \\
\hline 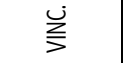 & 0 & 0 & 0 & 0 & 0 & 0 & 0 & 0 & 0 & 1 & 0 & 0 & 0 & 0 & 0 & 0 & 0 & 0 & 0 & 0 & 0 & 0 & 0 & 0 \\
\hline 总 & 0 & 0 & 0 & 2 & 0 & 0 & 0 & 0 & 0 & 4 & 0 & 0 & 0 & 0 & 0 & 0 & 0 & 0 & 0 & 0 & 0 & 0 & 0 & 0 \\
\hline 亗离出 & 0 & 0 & 0 & 0 & 0 & 0 & 0 & 0 & 0 & 2 & 0 & 0 & 0 & 0 & 0 & 0 & 0 & 0 & 0 & 0 & 1 & 0 & 0 & 0 \\
\hline 引这 & 0 & 0 & 0 & 0 & 0 & 0 & 0 & 0 & 0 & 3 & 0 & 0 & 0 & 0 & 0 & 0 & 0 & 0 & 0 & 0 & 1 & 0 & 0 & 0 \\
\hline$\frac{z}{6} 5$ & 5 & 1 & 2 & 39 & 3 & 1 & 2 & 3 & 3 & 111 & 22 & 2 & 2 & 11 & 5 & 2 & 5 & 22 & 11 & 1 & 18 & 3 & 4 & 4 \\
\hline
\end{tabular}




\begin{tabular}{|c|c|c|c|c|c|c|c|c|c|c|c|c|c|c|c|c|c|c|c|c|c|c|c|c|c|}
\hline & $\begin{array}{l}\text { L. SIMB. } \\
\text { LEL CAND. }\end{array}$ & $\begin{array}{c}\text { MACROSISTE } \\
\text { MA }\end{array}$ & \begin{tabular}{|c} 
NAVEGACI \\
ON
\end{tabular} & NOCION & OBJETO & $\begin{array}{l}\text { OBT. } \\
\text { INF. }\end{array}$ & $\begin{array}{l}\text { ORG. } \\
\text { ESC. }\end{array}$ & PRECOND & $\begin{array}{l}\text { PPIO. } \\
\text { CIRC. }\end{array}$ & $\begin{array}{l}\text { PPIO. } \\
\text { VOC. } \\
\text { MIN. }\end{array}$ & RECURSO & $\begin{array}{c}\text { REQUISIT } \\
0\end{array}$ & $\begin{array}{l}\text { REQUI. } \\
\text { FUNC. }\end{array}$ & $\begin{array}{l}\text { REQUI. } \\
\text { NO } \\
\text { FUNC. }\end{array}$ & \begin{tabular}{|l} 
RESTR. \\
.
\end{tabular} & $\begin{array}{c}\text { SIMB. } \\
\text { LEL }\end{array}$ & $\begin{array}{l}\text { SIST. } \\
\text { SOFT. }\end{array}$ & SUBESC. & SUIETO & $\begin{array}{c}\text { UB. } \\
\text { GEOGR. }\end{array}$ & $\begin{array}{c}\text { UB. } \\
\text { TEMP. }\end{array}$ & $\begin{array}{l}\text { UNIF. } \\
\text { ESC. }\end{array}$ & UdD & $\begin{array}{l}\text { VAL. } \\
\text { ESC. }\end{array}$ & VAL. LEL \\
\hline 产 & 0 & 0 & 0 & 0 & 0 & 0 & 0 & 0 & 0 & 0 & 0 & 0 & 0 & 0 & 0 & 1 & 0 & 0 & 1 & 0 & 0 & 0 & 0 & 0 & 0 \\
\hline 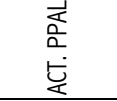 & 0 & 2 & 0 & 0 & 0 & 0 & 0 & 0 & 0 & 0 & 0 & 0 & 0 & 0 & 0 & 1 & 0 & 0 & 1 & 0 & 0 & 0 & 0 & 0 & 0 \\
\hline 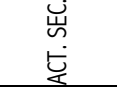 & 0 & 3 & 0 & 0 & 0 & 0 & 0 & 0 & 0 & 0 & 0 & 0 & 0 & 0 & 0 & 1 & 0 & 0 & 1 & 0 & 0 & 0 & 0 & 0 & 0 \\
\hline 产岁总 & 0 & 0 & 0 & 0 & 0 & 0 & 0 & 0 & 0 & 0 & 0 & 0 & 0 & 0 & 0 & 0 & 0 & 0 & 0 & 0 & 0 & 0 & 0 & 0 & 0 \\
\hline 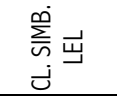 & 0 & 0 & 0 & 1 & 0 & 0 & 0 & 0 & 0 & 0 & 0 & 0 & 0 & 0 & 0 & 3 & 0 & 0 & 0 & 0 & 0 & 0 & 0 & 0 & 0 \\
\hline 己定 & 0 & 0 & 0 & 0 & 1 & 0 & 0 & 0 & 0 & 0 & 0 & 0 & 0 & 0 & 0 & 1 & 0 & 0 & 1 & 0 & 0 & 0 & 0 & 0 & 0 \\
\hline ن & 0 & 0 & 0 & 0 & 0 & 0 & 0 & 0 & 0 & 0 & 0 & 0 & 0 & 0 & 0 & 1 & 0 & 0 & 0 & 0 & 0 & 0 & 2 & 0 & 0 \\
\hline $\begin{array}{l}\text { 岑䈉 } \\
\text { 急 }\end{array}$ & 0 & 0 & 0 & 0 & 0 & 0 & 0 & 0 & 0 & 0 & 0 & 0 & 0 & 0 & 0 & 0 & 0 & 0 & 0 & 0 & 0 & 0 & 1 & 1 & 1 \\
\hline 咅安 & 0 & 0 & 0 & 0 & 0 & 0 & 0 & 0 & 0 & 0 & 0 & 0 & 0 & 0 & 0 & 0 & 0 & 0 & 0 & 0 & 0 & 0 & 0 & 0 & 0 \\
\hline 竘窇。 & 0 & 0 & 0 & 0 & 0 & 0 & 0 & 1 & 0 & 0 & 0 & 0 & 0 & 0 & 0 & 0 & 0 & 0 & 0 & 1 & 1 & 0 & 0 & 0 & 0 \\
\hline 产言 & 0 & 0 & 0 & 0 & 0 & 0 & 0 & 0 & 0 & 0 & 0 & 0 & 0 & 0 & 0 & 0 & 0 & 0 & 0 & 0 & 0 & 0 & 0 & 0 & 0 \\
\hline 言总产 & 0 & 0 & 0 & 0 & 0 & 0 & 0 & 0 & 0 & 0 & 0 & 0 & 0 & 0 & 0 & 0 & 0 & 0 & 0 & 0 & 0 & 0 & 0 & 0 & 0 \\
\hline 咅芉 & 0 & 0 & 0 & 0 & 0 & 0 & 0 & 0 & 0 & 0 & 0 & 0 & 0 & 0 & 0 & 0 & 0 & 0 & 0 & 0 & 0 & 0 & 0 & 0 & 0 \\
\hline$\sum_{\bar{\delta}}^{0} \overrightarrow{8}$ & 0 & 0 & 0 & 0 & 0 & 0 & 0 & 0 & 0 & 0 & 0 & 0 & 0 & 0 & 0 & 0 & 0 & 0 & 0 & 0 & 0 & 0 & 0 & 0 & 0 \\
\hline$\sum_{\bar{c}}^{0}$ & 0 & 0 & 0 & 0 & 0 & 0 & 0 & 0 & 0 & 0 & 3 & 0 & 0 & 0 & 0 & 0 & 0 & 0 & 0 & 0 & 0 & 0 & 0 & 0 & 0 \\
\hline
\end{tabular}




\begin{tabular}{|c|c|c|c|c|c|c|c|c|c|c|c|c|c|c|c|c|c|c|c|c|c|c|c|c|c|}
\hline & $\begin{array}{l}\text { L. SIMB. } \\
\text { LEL CAND. }\end{array}$ & $\begin{array}{c}\text { MACROSISTE } \\
\text { MA }\end{array}$ & $\begin{array}{c}\text { NAVEGACI } \\
\text { ON }\end{array}$ & NOCION & OBJETO & $\begin{array}{l}\text { OBT. } \\
\text { INF. }\end{array}$ & $\begin{array}{l}\text { ORG. } \\
\text { ESC. }\end{array}$ & \begin{tabular}{|c|} 
PRECOND \\
\end{tabular} & $\begin{array}{l}\text { PPIO. } \\
\text { CIRC. }\end{array}$ & $\begin{array}{l}\text { PPIO. } \\
\text { VOC. } \\
\text { MIN. }\end{array}$ & RECURSO & $\begin{array}{c}\text { REQUISIT } \\
0\end{array}$ & $\begin{array}{l}\text { REQUI. } \\
\text { FUNC. }\end{array}$ & $\begin{array}{c}\text { REQUI. } \\
\text { NO } \\
\text { FUNC. }\end{array}$ & RESTR. & $\begin{array}{c}\text { SIMB. } \\
\text { LEL }\end{array}$ & $\begin{array}{l}\text { SIST. } \\
\text { SOFT. }\end{array}$ & SUBESC. & SUJETO & $\begin{array}{l}\text { UB. } \\
\text { GEOGR. }\end{array}$ & $\begin{array}{l}\text { UB. } \\
\text { TEMP. }\end{array}$ & $\begin{array}{l}\text { UNIF. } \\
\text { ESC. }\end{array}$ & UdD & $\begin{array}{l}\text { VAL. } \\
\text { ESC. }\end{array}$ & VAL. LEL \\
\hline 咅总 & 0 & 0 & 0 & 0 & 0 & 0 & 0 & 0 & 0 & 0 & 0 & 0 & 0 & 0 & 0 & 0 & 0 & 1 & 0 & 0 & 0 & 0 & 0 & 0 & 0 \\
\hline 忘品 & 0 & 1 & 0 & 0 & 0 & 0 & 1 & 0 & 0 & 0 & 0 & 0 & 0 & 0 & 0 & 0 & 0 & 0 & 1 & 0 & 0 & 0 & 1 & 1 & 0 \\
\hline 咅 & 0 & 0 & 0 & 0 & 0 & 1 & 0 & 0 & 0 & 0 & 0 & 0 & 0 & 0 & 0 & 1 & 0 & 0 & 0 & 0 & 0 & 0 & 0 & 0 & 1 \\
\hline 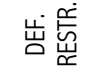 & 0 & 0 & 0 & 0 & 0 & 0 & 0 & 1 & 0 & 0 & 1 & 0 & 0 & 0 & 3 & 0 & 0 & 0 & 0 & 0 & 1 & 1 & 0 & 0 & 0 \\
\hline 密出嵒 & 0 & 1 & 0 & 0 & 1 & 0 & 0 & 1 & 0 & 0 & 2 & 0 & 0 & 0 & 0 & 12 & 0 & 0 & 5 & 1 & 1 & 0 & 0 & 0 & 0 \\
\hline 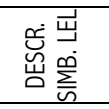 & 0 & 0 & 0 & 1 & 0 & 0 & 0 & 0 & 0 & 0 & 0 & 0 & 0 & 0 & 0 & 2 & 0 & 0 & 0 & 0 & 0 & 0 & 1 & 0 & 0 \\
\hline 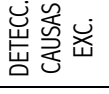 & 0 & 0 & 0 & 0 & 0 & 0 & 0 & 0 & 0 & 0 & 0 & 0 & 0 & 0 & 1 & 0 & 0 & 0 & 0 & 0 & 0 & 0 & 0 & 0 & 0 \\
\hline 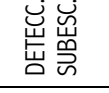 & 0 & 0 & 0 & 0 & 0 & 0 & 0 & 0 & 0 & 0 & 0 & 0 & 0 & 0 & 0 & 0 & 0 & 0 & 0 & 0 & 0 & 0 & 0 & 0 & 0 \\
\hline 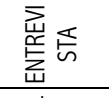 & 0 & 1 & 0 & 0 & 0 & 0 & 0 & 0 & 0 & 0 & 0 & 0 & 0 & 0 & 0 & 0 & 0 & 0 & 0 & 0 & 0 & 0 & 0 & 1 & 1 \\
\hline 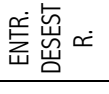 & 0 & 0 & 0 & 0 & 0 & 0 & 0 & 0 & 0 & 0 & 0 & 0 & 0 & 0 & 0 & 0 & 0 & 0 & 0 & 0 & 0 & 0 & 1 & 0 & 0 \\
\hline 站密 & 0 & 0 & 0 & 0 & 0 & 0 & 0 & 0 & 0 & 0 & 0 & 0 & 0 & 0 & 0 & 0 & 0 & 0 & 0 & 0 & 0 & 0 & 1 & 1 & 1 \\
\hline 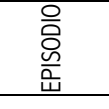 & 0 & 0 & 0 & 0 & 0 & 0 & 0 & 0 & 0 & 0 & 1 & 0 & 1 & 0 & 1 & 0 & 0 & 0 & 0 & 0 & 0 & 0 & 0 & 0 & 0 \\
\hline : & 0 & 1 & 0 & 0 & 0 & 0 & 0 & 1 & 0 & 0 & 0 & 0 & 0 & 0 & 0 & 0 & 0 & 0 & 0 & 0 & 0 & 0 & 0 & 0 & 0 \\
\hline $\begin{array}{l}\dot{y} \\
\dot{0} \\
\dot{\tilde{u}} \\
\end{array}$ & 0 & 0 & 0 & 0 & 0 & 0 & 0 & 0 & 0 & 0 & 0 & 0 & 0 & 0 & 0 & 0 & 0 & 0 & 0 & 0 & 0 & 0 & 0 & 0 & 0 \\
\hline 它离 & 0 & 0 & 0 & 0 & 0 & 0 & 0 & 0 & 0 & 0 & 0 & 0 & 0 & 0 & 0 & 0 & 0 & 0 & 0 & 0 & 0 & 0 & 0 & 0 & 0 \\
\hline $\begin{array}{l}\text { O. } \\
\text { 恙 } \\
\text { 出 }\end{array}$ & 0 & 0 & 0 & 0 & 0 & 0 & 0 & 0 & 0 & 0 & 0 & 0 & 0 & 0 & 0 & 0 & 0 & 0 & 0 & 0 & 0 & 0 & 0 & 0 & 0 \\
\hline
\end{tabular}




\begin{tabular}{|c|c|c|c|c|c|c|c|c|c|c|c|c|c|c|c|c|c|c|c|c|c|c|c|c|c|}
\hline & $\begin{array}{l}\text { L. SIMB } \\
\text { LEL CAND. }\end{array}$ & $\begin{array}{c}\text { MACROSISTE } \\
\text { MA }\end{array}$ & $\begin{array}{l}\text { NAVEGACI } \\
\text { ON }\end{array}$ & NOCION & OBJETO & $\begin{array}{l}\text { OBT. } \\
\text { INF. }\end{array}$ & $\begin{array}{l}\text { ORG. } \\
\text { ESC. }\end{array}$ & PRECOND & $\begin{array}{l}\text { PPIO. } \\
\text { CIIC. }\end{array}$ & $\begin{array}{l}\text { PPIO. } \\
\text { VOC. } \\
\text { MIN. }\end{array}$ & RECURSO & $\begin{array}{c}\text { REQUISIT } \\
0\end{array}$ & \begin{tabular}{|l} 
REQUI. \\
FUNC.
\end{tabular} & \begin{tabular}{|c} 
REQUI. \\
NO \\
FUNC.
\end{tabular} & RESTR. & $\begin{array}{c}\text { SIMB. } \\
\text { LEL }\end{array}$ & \begin{tabular}{|l|} 
SIST. \\
SOFT.
\end{tabular} & SUBESC. & SUEETO & $\begin{array}{c}\text { UB. } \\
\text { GEOGR. }\end{array}$ & $\begin{array}{l}\text { UB. } \\
\text { TEMP. }\end{array}$ & $\begin{array}{l}\text { UNIF. } \\
\text { ESC. }\end{array}$ & UdD & $\begin{array}{l}\text { VAL. } \\
\text { ESC. }\end{array}$ & VAL. LEL \\
\hline $\begin{array}{l}\text { 出 } \\
0 \\
\vdots \\
\end{array}$ & 0 & 0 & 0 & 0 & 0 & 0 & 0 & 0 & 0 & 0 & 0 & 0 & 0 & 0 & 0 & 0 & 0 & 0 & 0 & 0 & 0 & 0 & 0 & 0 & 0 \\
\hline 岀 & 0 & 2 & 0 & 0 & 0 & 0 & 0 & 0 & 0 & 0 & 0 & 0 & 0 & 0 & 0 & 2 & 1 & 1 & 0 & 0 & 0 & 0 & 0 & 0 & 0 \\
\hline 岁密 & 0 & 1 & 0 & 0 & 0 & 0 & 0 & 0 & 0 & 0 & 0 & 0 & 0 & 0 & 0 & 0 & 0 & 0 & 0 & 0 & 0 & 1 & 0 & 1 & 0 \\
\hline 岀完 & 0 & 1 & 0 & 0 & 0 & 0 & 0 & 0 & 0 & 0 & 0 & 0 & 0 & 0 & 0 & 0 & 0 & 0 & 0 & 0 & 0 & 0 & 0 & 0 & 0 \\
\hline $\begin{array}{l}\text { 量 } \\
\text { 点 }\end{array}$ & 0 & 0 & 0 & 1 & 1 & 0 & 0 & 0 & 0 & 0 & 0 & 0 & 0 & 0 & 0 & 2 & 0 & 0 & 1 & 0 & 0 & 0 & 0 & 0 & 0 \\
\hline $\begin{array}{l}\text { 음 } \\
\text { 岀z } \\
\text { y. }\end{array}$ & 0 & 0 & 0 & 0 & 0 & 0 & 0 & 0 & 0 & 0 & 0 & 0 & 0 & 0 & 0 & 0 & 0 & 0 & 0 & 0 & 0 & 0 & 0 & 0 & 0 \\
\hline 至出定 & 0 & 0 & 0 & 0 & 0 & 0 & 0 & 0 & 0 & 0 & 0 & 0 & 0 & 0 & 0 & 0 & 0 & 0 & 1 & 0 & 0 & 0 & 0 & 0 & 0 \\
\hline 怠离雭 & 1 & 0 & 0 & 0 & 0 & 1 & 0 & 0 & 0 & 0 & 0 & 0 & 0 & 0 & 0 & 1 & 0 & 0 & 0 & 0 & 0 & 0 & 1 & 0 & 0 \\
\hline 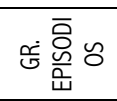 & 0 & 0 & 0 & 0 & 0 & 0 & 0 & 0 & 0 & 0 & 0 & 0 & 0 & 0 & 0 & 0 & 0 & 0 & 0 & 0 & 0 & 0 & 0 & 0 & 0 \\
\hline 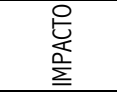 & 0 & 2 & 0 & 0 & 0 & 0 & 0 & 0 & 1 & 1 & 0 & 0 & 0 & 0 & 0 & 5 & 0 & 0 & 1 & 0 & 0 & 0 & 0 & 0 & 0 \\
\hline 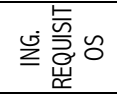 & 0 & 0 & 0 & 0 & 0 & 1 & 1 & 0 & 0 & 0 & 0 & 0 & 0 & 0 & 0 & 0 & 0 & 0 & 0 & 0 & 0 & 1 & 1 & 1 & 1 \\
\hline 䍃出 & 0 & 0 & 0 & 0 & 0 & 0 & 0 & 0 & 0 & 0 & 0 & 0 & 0 & 0 & 0 & 0 & 0 & 1 & 0 & 0 & 0 & 0 & 0 & 0 & 0 \\
\hline$\ddot{\exists}$ & 0 & 0 & 0 & 0 & 0 & 0 & 0 & 0 & 1 & 1 & 0 & 0 & 0 & 0 & 0 & 1 & 0 & 0 & 0 & 0 & 0 & 0 & 1 & 0 & 0 \\
\hline - 訇 & 0 & 0 & 0 & 0 & 0 & 0 & 0 & 0 & 0 & 0 & 0 & 0 & 0 & 0 & 0 & 1 & 0 & 0 & 0 & 0 & 0 & 0 & 0 & 0 & 1 \\
\hline 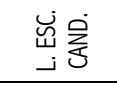 & 0 & 0 & 0 & 0 & 0 & 0 & 0 & 0 & 0 & 0 & 0 & 0 & 0 & 0 & 0 & 0 & 0 & 0 & 0 & 0 & 0 & 0 & 0 & 0 & 0 \\
\hline 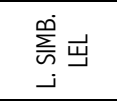 & 0 & 0 & 0 & 0 & 0 & 0 & 0 & 0 & 0 & 0 & 0 & 0 & 0 & 0 & 0 & 3 & 0 & 0 & 0 & 0 & 0 & 0 & 0 & 0 & 0 \\
\hline$\sum_{j}^{\infty} \Psi \sum_{j}^{\infty}$ & 0 & 0 & 0 & 0 & 0 & 0 & 0 & 0 & 0 & 0 & 0 & 0 & 0 & 0 & 0 & 4 & 0 & 0 & 0 & 0 & 0 & 0 & 2 & 0 & 0 \\
\hline
\end{tabular}




\begin{tabular}{|c|c|c|c|c|c|c|c|c|c|c|c|c|c|c|c|c|c|c|c|c|c|c|c|c|c|}
\hline & $\begin{array}{l}\text { L. SIMB } \\
\text { LEL CAND. }\end{array}$ & $\begin{array}{c}\text { MACROSISTT } \\
\text { MA }\end{array}$ & $\begin{array}{l}\text { NAVEGACI } \\
\text { ON }\end{array}$ & NOCION & OВЕЕТО & $\begin{array}{l}\text { OBT. } \\
\text { INF. }\end{array}$ & $\begin{array}{l}\text { ORG. } \\
\text { ESC. }\end{array}$ & PRECOND & $\begin{array}{l}\text { PPIO. } \\
\text { CIRC. }\end{array}$ & $\begin{array}{l}\text { PPIO. } \\
\text { VOC. } \\
\text { MIN. }\end{array}$ & RECURSO & $\begin{array}{c}\text { REQUISIT } \\
0\end{array}$ & $\begin{array}{l}\text { REQUI. } \\
\text { FUNC. }\end{array}$ & $\begin{array}{l}\text { REQUI. } \\
\text { NO } \\
\text { FUNC. }\end{array}$ & RESTR. & $\begin{array}{c}\text { SIMB. } \\
\text { LEL }\end{array}$ & $\begin{array}{l}\text { SIST. } \\
\text { SOFT. }\end{array}$ & SUBESC. & SUEETO & $\begin{array}{l}\text { UB. } \\
\text { GEOGR. }\end{array}$ & $\begin{array}{l}\text { UB. } \\
\text { TEMP. }\end{array}$ & $\begin{array}{l}\text { UNIF. } \\
\text { ESC. }\end{array}$ & UdD & $\begin{array}{l}\text { VAL. } \\
\text { ESC. }\end{array}$ & VAL. LEL \\
\hline 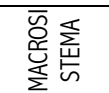 & 0 & 0 & 0 & 0 & 0 & 0 & 0 & 0 & 0 & 0 & 0 & 0 & 0 & 0 & 0 & 0 & 1 & 0 & 0 & 0 & 0 & 0 & 0 & 0 & 0 \\
\hline 嵒列 & 0 & 0 & 0 & 0 & 0 & 0 & 0 & 0 & 0 & 0 & 0 & 0 & 0 & 0 & 0 & 2 & 0 & 0 & 0 & 0 & 0 & 0 & 0 & 0 & 0 \\
\hline $\begin{array}{l}\text { zo } \\
\text { 을 } \\
\end{array}$ & 0 & 1 & 0 & 0 & 0 & 0 & 0 & 0 & 1 & 1 & 0 & 0 & 0 & 0 & 0 & 4 & 0 & 0 & 0 & 0 & 0 & 0 & 0 & 0 & 0 \\
\hline 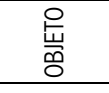 & 0 & 0 & 0 & 1 & 0 & 0 & 0 & 0 & 0 & 0 & 0 & 0 & 0 & 0 & 0 & 1 & 0 & 0 & 1 & 0 & 0 & 0 & 0 & 0 & 0 \\
\hline 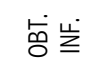 & 0 & 0 & 0 & 0 & 0 & 0 & 0 & 0 & 0 & 0 & 0 & 0 & 0 & 0 & 0 & 0 & 0 & 0 & 0 & 0 & 0 & 0 & 1 & 0 & 0 \\
\hline ن웡 & 0 & 0 & 0 & 0 & 0 & 0 & 0 & 0 & 0 & 0 & 0 & 0 & 0 & 0 & 1 & 0 & 0 & 0 & 0 & 0 & 0 & 1 & 0 & 0 & 0 \\
\hline 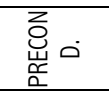 & 0 & 1 & 0 & 0 & 0 & 0 & 0 & 0 & 0 & 0 & 0 & 0 & 0 & 0 & 1 & 0 & 0 & 0 & 0 & 0 & 0 & 0 & 0 & 0 & 0 \\
\hline 음 & 0 & 0 & 1 & 0 & 0 & 0 & 0 & 0 & 0 & 0 & 0 & 0 & 0 & 0 & 0 & 3 & 0 & 0 & 0 & 0 & 0 & 0 & 0 & 0 & 0 \\
\hline 음 & 0 & 1 & 0 & 0 & 0 & 0 & 0 & 0 & 0 & 0 & 0 & 0 & 0 & 0 & 0 & 1 & 0 & 0 & 0 & 0 & 0 & 0 & 0 & 0 & 0 \\
\hline 筑。 & 0 & 0 & 0 & 0 & 1 & 0 & 0 & 0 & 0 & 0 & 0 & 0 & 0 & 0 & 1 & 1 & 0 & 0 & 0 & 0 & 0 & 0 & 0 & 0 & 0 \\
\hline 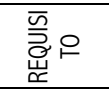 & 0 & 0 & 0 & 0 & 0 & 0 & 0 & 0 & 0 & 0 & 0 & 0 & 1 & 1 & 0 & 0 & 2 & 0 & 0 & 0 & 0 & 0 & 0 & 0 & 0 \\
\hline 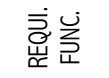 & 0 & 0 & 0 & 0 & 0 & 0 & 0 & 0 & 0 & 0 & 0 & 1 & 0 & 0 & 0 & 0 & 1 & 0 & 0 & 0 & 0 & 0 & 0 & 0 & 0 \\
\hline 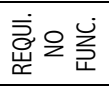 & 0 & 0 & 0 & 0 & 0 & 0 & 0 & 0 & 0 & 0 & 0 & 1 & 0 & 0 & 1 & 0 & 1 & 0 & 0 & 0 & 0 & 0 & 0 & 0 & 0 \\
\hline 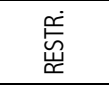 & 0 & 0 & 0 & 0 & 0 & 0 & 0 & 1 & 0 & 0 & 2 & 0 & 0 & 1 & 0 & 0 & 0 & 0 & 0 & 1 & 1 & 0 & 0 & 0 & 0 \\
\hline 离ت & 0 & 0 & 0 & 1 & 0 & 0 & 0 & 0 & 1 & 1 & 0 & 0 & 0 & 0 & 0 & 0 & 0 & 0 & 0 & 0 & 0 & 0 & 2 & 0 & 0 \\
\hline 宓怠 & 0 & 0 & 0 & 0 & 0 & 0 & 0 & 0 & 0 & 0 & 0 & 1 & 0 & 0 & 0 & 0 & 0 & 0 & 0 & 0 & 0 & 0 & 1 & 0 & 0 \\
\hline $\begin{array}{l}\text { 岕 } \\
\text { 容 }\end{array}$ & 0 & 0 & 0 & 0 & 0 & 0 & 0 & 0 & 0 & 0 & 0 & 0 & 0 & 0 & 0 & 0 & 0 & 0 & 0 & 0 & 0 & 1 & 0 & 0 & 0 \\
\hline 总 & 0 & 1 & 0 & 1 & 1 & 0 & 0 & 0 & 0 & 0 & 0 & 0 & 0 & 0 & 0 & 2 & 0 & 0 & 0 & 0 & 0 & 0 & 0 & 0 & 0 \\
\hline
\end{tabular}




\begin{tabular}{|c|c|c|c|c|c|c|c|c|c|c|c|c|c|c|c|c|c|c|c|c|c|c|c|c|c|}
\hline & $\begin{array}{l}\text { L. SIMB. } \\
\text { LEL CAND. }\end{array}$ & $\begin{array}{c}\text { MACROSISTE } \\
\text { MA }\end{array}$ & \begin{tabular}{|c} 
NAVEGACI \\
ON
\end{tabular} & NOCION & OВЕЕТО & $\begin{array}{l}\text { OBT. } \\
\text { INF. }\end{array}$ & $\begin{array}{l}\text { ORG. } \\
\text { ESC. }\end{array}$ & PRECOND & $\begin{array}{l}\text { PPIO. } \\
\text { CIRC. }\end{array}$ & $\begin{array}{l}\text { PPIO. } \\
\text { VOC. } \\
\text { MIN. }\end{array}$ & RECURSO & \begin{tabular}{|c|} 
REQUISIT \\
0
\end{tabular} & $\begin{array}{l}\text { REQUI. } \\
\text { FUNC. }\end{array}$ & $\begin{array}{c}\text { REQUI. } \\
\text { NO } \\
\text { FUNC. }\end{array}$ & RESTR. & $\begin{array}{c}\text { SIMB. } \\
\text { LEL }\end{array}$ & $\begin{array}{l}\text { SIST. } \\
\text { SOFT. }\end{array}$ & SUBESC. & SUEETO & $\begin{array}{c}\text { UB. } \\
\text { GEOGR. }\end{array}$ & $\begin{array}{c}\text { UB. } \\
\text { TEMP. }\end{array}$ & $\begin{array}{l}\text { UNIF. } \\
\text { ESC. }\end{array}$ & UdD & $\begin{array}{l}\text { VAL. } \\
\text { ESC. }\end{array}$ & VAL. LEL \\
\hline 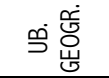 & 0 & 0 & 0 & 0 & 0 & 0 & 0 & 0 & 0 & 0 & 0 & 0 & 0 & 0 & 1 & 0 & 0 & 0 & 0 & 0 & 0 & 0 & 0 & 0 & 0 \\
\hline 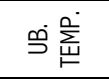 & 0 & 0 & 0 & 0 & 0 & 0 & 0 & 0 & 0 & 0 & 0 & 0 & 0 & 0 & 1 & 0 & 0 & 0 & 0 & 0 & 0 & 0 & 0 & 0 & 0 \\
\hline 訔出 & 0 & 0 & 0 & 0 & 0 & 0 & 0 & 0 & 0 & 0 & 0 & 0 & 0 & 0 & 0 & 0 & 0 & 0 & 0 & 0 & 0 & 0 & 0 & 0 & 0 \\
\hline 윅 & 0 & 2 & 0 & 0 & 0 & 1 & 0 & 0 & 0 & 0 & 0 & 1 & 0 & 0 & 0 & 0 & 0 & 0 & 0 & 0 & 0 & 0 & 0 & 0 & 0 \\
\hline 蒿出 & 0 & 0 & 0 & 0 & 0 & 0 & 0 & 0 & 0 & 0 & 0 & 0 & 0 & 0 & 0 & 0 & 0 & 0 & 0 & 0 & 0 & 0 & 0 & 0 & 0 \\
\hline$\dot{\dot{\$}} \vec{\exists}$ & 0 & 0 & 0 & 0 & 0 & 1 & 0 & 0 & 0 & 0 & 0 & 0 & 0 & 0 & 0 & 1 & 0 & 0 & 0 & 0 & 0 & 0 & 1 & 0 & 0 \\
\hline 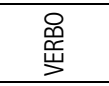 & 0 & 1 & 0 & 1 & 0 & 0 & 0 & 0 & 0 & 0 & 0 & 0 & 0 & 0 & 1 & 2 & 0 & 0 & 1 & 0 & 0 & 0 & 0 & 0 & 0 \\
\hline 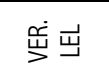 & 0 & 0 & 0 & 0 & 0 & 0 & 0 & 0 & 1 & 1 & 0 & 0 & 0 & 0 & 0 & 2 & 0 & 0 & 1 & 0 & 0 & 0 & 0 & 0 & 0 \\
\hline$\breve{ٍ ~}$ & 0 & 0 & 1 & 0 & 0 & 0 & 0 & 0 & 0 & 0 & 0 & 0 & 0 & 0 & 0 & 1 & 0 & 0 & 1 & 0 & 0 & 0 & 0 & 0 & 0 \\
\hline 站 & 0 & 0 & 1 & 0 & 0 & 0 & 0 & 0 & 0 & 0 & 0 & 0 & 0 & 0 & 0 & & 0 & 0 & 1 & 0 & 0 & 0 & 0 & 0 & 0 \\
\hline 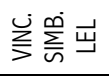 & 0 & 0 & 2 & 0 & 0 & 0 & 0 & 0 & 0 & 0 & 0 & 0 & 0 & 0 & 0 & 5 & 0 & 0 & 1 & 0 & 0 & 0 & 0 & 0 & 0 \\
\hline 岁昱 & 0 & 0 & 1 & 0 & 0 & 0 & 0 & 0 & 0 & 0 & 0 & 0 & 0 & 0 & 0 & 2 & 0 & 0 & 1 & 0 & 0 & 0 & 0 & 0 & 0 \\
\hline $\begin{array}{l}2 \\
25 \\
4\end{array}$ & 1 & 22 & 6 & 7 & 5 & 5 & 2 & 5 & 5 & 5 & 9 & 4 & 2 & 2 & 12 & 69 & 6 & 3 & 15 & 3 & 4 & 5 & 17 & 6 & 6 \\
\hline
\end{tabular}




\begin{tabular}{|c|c|c|c|c|c|c|c|}
\hline & VERBO & VER. LEL & VINC. & $\begin{array}{l}\text { VINC. } \\
\text { ESC. }\end{array}$ & $\begin{array}{l}\text { VINC. } \\
\text { SIMB. } \\
\text { LEL }\end{array}$ & $\begin{array}{l}\text { VINC. } \\
\text { ARB. }\end{array}$ & $\begin{array}{c}\text { FAN } \\
\text { IN }\end{array}$ \\
\hline 总 & 0 & 0 & 0 & 0 & 0 & 0 & 6 \\
\hline 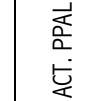 & 0 & 0 & 0 & 0 & 0 & 0 & 8 \\
\hline 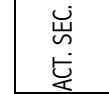 & 0 & 0 & 0 & 0 & 0 & 0 & 9 \\
\hline 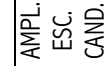 & 0 & 0 & 0 & 0 & 0 & 0 & 10 \\
\hline$\sum_{\sum_{\mathcal{U}}^{\infty}}^{\infty}$ & 0 & 0 & 0 & 0 & 0 & 0 & 9 \\
\hline 己寄 & 1 & 0 & 0 & 0 & 0 & 0 & 7 \\
\hline ن & 0 & 0 & 0 & 0 & 0 & 0 & 5 \\
\hline 岑䍃 & 0 & 0 & 0 & 0 & 0 & 0 & 5 \\
\hline 产安 & 0 & 0 & 0 & 0 & 0 & 0 & 7 \\
\hline 总產 & 0 & 0 & 0 & 0 & 0 & 0 & 11 \\
\hline 爰产 & 0 & 0 & 0 & 0 & 0 & 0 & 4 \\
\hline 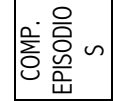 & 0 & 0 & 0 & 0 & 0 & 0 & 13 \\
\hline 章岑 & 0 & 0 & 0 & 0 & 0 & 0 & 11 \\
\hline 這审 & 0 & 0 & 0 & 0 & 0 & 0 & 5 \\
\hline 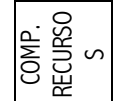 & 0 & 0 & 0 & 0 & 0 & 0 & 8 \\
\hline
\end{tabular}




\begin{tabular}{|c|c|c|c|c|c|c|c|}
\hline & VERBO & VER. LEL & VINC. & $\begin{array}{l}\text { VINC. } \\
\text { ESC. }\end{array}$ & $\begin{array}{l}\text { VINC. } \\
\text { SIMB. } \\
\text { LEL }\end{array}$ & $\begin{array}{l}\text { VINC. } \\
\text { ARB. }\end{array}$ & $\begin{array}{l}\text { FAN } \\
\text { IN }\end{array}$ \\
\hline 穵兑 & 0 & 0 & 0 & 0 & 0 & 0 & 7 \\
\hline 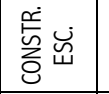 & 0 & 0 & 0 & 0 & 0 & 0 & 14 \\
\hline 㝘山 & 0 & 1 & 0 & 0 & 0 & 0 & 8 \\
\hline 岀怠 & 0 & 0 & 0 & 0 & 0 & 0 & 12 \\
\hline 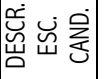 & 3 & 0 & 0 & 0 & 0 & 0 & 59 \\
\hline 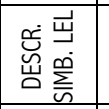 & 0 & 0 & 0 & 0 & 0 & 0 & 5 \\
\hline | & 0 & 0 & 0 & 0 & 0 & 0 & 7 \\
\hline 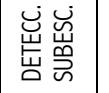 & 0 & 0 & 0 & 0 & 0 & 0 & 11 \\
\hline 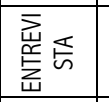 & 0 & 0 & 0 & 0 & 0 & 0 & 10 \\
\hline 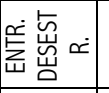 & 0 & 0 & 0 & 0 & 0 & 0 & 5 \\
\hline 点站 & 0 & 0 & 0 & 0 & 0 & 0 & 10 \\
\hline 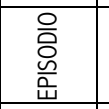 & 0 & 0 & 0 & 0 & 0 & 0 & 17 \\
\hline 妾 & 0 & 0 & 0 & 0 & 0 & 0 & 12 \\
\hline $\begin{array}{l}\dot{\Delta} \\
\dot{0} \\
\dot{\tilde{u}} \\
\end{array}$ & 0 & 0 & 0 & 0 & 0 & 0 & 4 \\
\hline 号 & 0 & 0 & 0 & 0 & 0 & 0 & 3 \\
\hline
\end{tabular}




\begin{tabular}{|c|c|c|c|c|c|c|c|}
\hline & VERBO & VER. LEL & VINC. & $\begin{array}{l}\text { VINC. } \\
\text { ESC. }\end{array}$ & $\begin{array}{l}\text { VINC. } \\
\text { SIMB. } \\
\text { LEL }\end{array}$ & $\begin{array}{l}\text { VINC. } \\
\text { ARB. }\end{array}$ & $\begin{array}{l}\text { FAN } \\
\text { IN }\end{array}$ \\
\hline 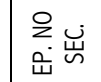 & 0 & 0 & 0 & 0 & 0 & 0 & 4 \\
\hline 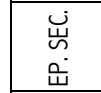 & 0 & 0 & 0 & 0 & 0 & 0 & 4 \\
\hline 峞 & 0 & 0 & 1 & 0 & 0 & 0 & 16 \\
\hline 㟒豈 & 0 & 0 & 0 & 0 & 0 & 0 & 7 \\
\hline 岀完 & 0 & 0 & 0 & 0 & 0 & 0 & 12 \\
\hline $\begin{array}{l}\text { 足 } \\
\text { 莣 } \\
\end{array}$ & 0 & 0 & 0 & 0 & 0 & 0 & 6 \\
\hline $\begin{array}{l}\text { 을 } \\
\text { 岀z } \\
\text { y. }\end{array}$ & 0 & 0 & 0 & 0 & 0 & 0 & 7 \\
\hline 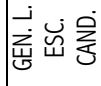 & 0 & 0 & 0 & 0 & 0 & 0 & 13 \\
\hline |خ & 0 & 0 & 0 & 0 & 0 & 0 & 6 \\
\hline 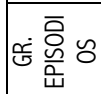 & 0 & 0 & 0 & 0 & 0 & 0 & 4 \\
\hline 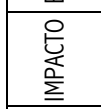 & 0 & 0 & 0 & 0 & 0 & 0 & 12 \\
\hline 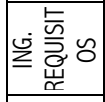 & 0 & 1 & 0 & 0 & 0 & 0 & 25 \\
\hline 总出 & 0 & 0 & 1 & 0 & 0 & 0 & 8 \\
\hline 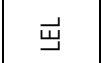 & 0 & 0 & 0 & 0 & 1 & 0 & 8 \\
\hline - 訔 & 0 & 0 & 0 & 0 & 0 & 0 & 3 \\
\hline 总害 & 0 & 0 & 0 & 0 & 0 & 0 & 4 \\
\hline
\end{tabular}




\begin{tabular}{|c|c|c|c|c|c|c|c|}
\hline & VERBO & VER. LEL & VINC. & $\begin{array}{l}\text { VIINC. } \\
\text { ESC. }\end{array}$ & $\begin{array}{l}\text { VINC. } \\
\text { SIMB. } \\
\text { LEL }\end{array}$ & $\begin{array}{l}\text { VINC. } \\
\text { ARB. }\end{array}$ & $\begin{array}{c}\text { FAN } \\
\text { IN }\end{array}$ \\
\hline 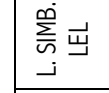 & 0 & 0 & 0 & 0 & 0 & 0 & 6 \\
\hline 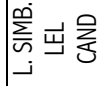 & 0 & 0 & 0 & 0 & 0 & 0 & 8 \\
\hline 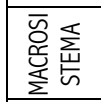 & 0 & 0 & 0 & 0 & 0 & 0 & 3 \\
\hline 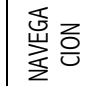 & 0 & 0 & 2 & 0 & 0 & 0 & 8 \\
\hline $\begin{array}{l}\text { zo } \\
\text { o } \\
z\end{array}$ & 0 & 0 & 0 & 0 & 0 & 0 & 7 \\
\hline $\begin{array}{l}\stackrel{ }{\mathrm{U}} \\
\mathrm{g}\end{array}$ & 1 & 0 & 0 & 0 & 0 & 0 & 5 \\
\hline 总檪 & 0 & 0 & 0 & 0 & 0 & 0 & 3 \\
\hline 岁 & 0 & 0 & 0 & 0 & 0 & 0 & 7 \\
\hline 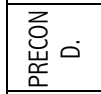 & 0 & 0 & 0 & 0 & 0 & 0 & 8 \\
\hline 을 岇 & 0 & 0 & 0 & 0 & 0 & 0 & 5 \\
\hline 品 & 0 & 0 & 0 & 0 & 0 & 0 & 4 \\
\hline 篆。 & 0 & 0 & 0 & 0 & 0 & 0 & 7 \\
\hline 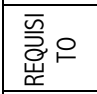 & 0 & 0 & 0 & 0 & 0 & 0 & 7 \\
\hline 䓌岕 & 0 & 0 & 0 & 0 & 0 & 0 & 4 \\
\hline 总说妾 & 0 & 0 & 0 & 0 & 0 & 0 & 4 \\
\hline 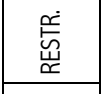 & 0 & 0 & 0 & 0 & 0 & 0 & 11 \\
\hline 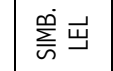 & 0 & 0 & 0 & 0 & 2 & 0 & 11 \\
\hline
\end{tabular}




\begin{tabular}{|c|c|c|c|c|c|c|c|}
\hline & VERBO & VER. LEL & VINC. & $\begin{array}{l}\text { VINC. } \\
\text { ESC. }\end{array}$ & $\begin{array}{l}\text { VINC. } \\
\text { SIMB. } \\
\text { LEL }\end{array}$ & $\begin{array}{l}\text { VINC. } \\
\text { ARB. }\end{array}$ & $\begin{array}{c}\text { FAN } \\
\text { IN }\end{array}$ \\
\hline 离离 & 0 & 0 & 0 & 0 & 0 & 0 & 3 \\
\hline $\begin{array}{l}\text { 岕 } \\
\text { 岕 }\end{array}$ & 1 & 0 & 0 & 0 & 0 & 0 & 11 \\
\hline 誉 & 0 & 0 & 0 & 0 & 0 & 0 & 7 \\
\hline 宇 & 0 & 0 & 0 & 0 & 0 & 0 & 3 \\
\hline$\stackrel{9}{9} \sum_{\tilde{H}}^{0}$ & 0 & 0 & 0 & 0 & 0 & 0 & 3 \\
\hline 訔出 & 0 & 0 & 0 & 0 & 0 & 0 & 9 \\
\hline 윟 & 0 & 0 & 0 & 0 & 0 & 0 & 5 \\
\hline نُ & 0 & 0 & 0 & 0 & 0 & 0 & 9 \\
\hline$\dot{\vec{\Sigma}} \overrightarrow{\mathrm{J}}$ & 0 & 0 & 0 & 0 & 0 & 0 & 8 \\
\hline $\begin{array}{l}\text { 尊 } \\
\text { 䍃 }\end{array}$ & 0 & 0 & 0 & 0 & 0 & 0 & 11 \\
\hline 辤出 & 0 & 0 & 0 & 0 & 0 & 0 & 5 \\
\hline 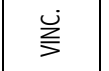 & 0 & 0 & 0 & 1 & 1 & 1 & 6 \\
\hline 岁出 & 0 & 0 & 2 & 0 & 0 & 0 & 11 \\
\hline 产离山 & 0 & 0 & 0 & 1 & 0 & 0 & 11 \\
\hline 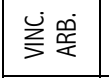 & 0 & 0 & 1 & 0 & 0 & 0 & 9 \\
\hline $\begin{array}{ll}\begin{array}{l}z \\
<\end{array} \\
4\end{array}$ & 6 & 2 & 7 & 2 & 4 & 1 & \\
\hline
\end{tabular}




\section{APENDICE A}

\section{CLASES DE PALABRAS}

Desde el punto de vista semántico y morfológico son clases de palabras el sustantivo, el adjetivo, el verbo, el adverbio, las palabras coordinativas (conjunciones y preposiciones), el artículo y los pronombres [Enciclopedia '90].

$\checkmark$ Sustantivo: es aquella clase de palabra que sirve para designar seres, personas o cosas que tienen existencia independiente, ya sea en la realidad, por abstracción o personificación.

Ejemplo: perro, Brasil, metáfora, etc.

$\checkmark$ Adjetivo: desde el punto de vista semántico es la parte de la oración que se añade al sustantivo para calificarlo o atribuirle diferentes tipos de propiedades.

Ejemplo: todos, algún, grande, etc.

$\checkmark$ Artículo: es un modificador directo del sustantivo y cumple con respecto a él funciones de determinación.

Ellos son: el, la, los, las, lo, uno, una, unos, unas [Enciclopedia '90].

$\checkmark$ Pronombre: es una clase de palabra no descriptiva y de significación ocasional orientada por circunstancias lingüísticas (el coloquio o el hilo del discurso).

Son algunos ejemplos de pronombres: yo, tú, él, que, donde, cual, cada, cuanto, cuyo, quien, este, ese, aquel, estos, esos, aquellos, su, sus, mucho, poco, ningún, etc. (esta lista no es exhaustiva) [Enciclopedia '90].

$\checkmark$ Verbo: es la parte de la oración que expresa actividad, pasión o estado, resulta que eso lo convierte en predicado integral $y$, en consecuencia, elemento central en toda oración [Enciclopedia '90]. El modo de un verbo depende de la formulación lógica del juicio y expresa si la acción del verbo tiene realidad o si depende de alguna condición o del deseo de alguien [Enciclopedia Labor '62]. Además de ello, por su semántica, los verbos suelen denotar aspectos de la realidad que son centrales en una descripción, ya se trate de ámbitos de actividad o ámbitos de estados. Es por esto que se considera a los verbos incluidos en una definición como un indicador de la funcionalidad y por lo tanto de la importancia del concepto en el que intervienen.

Las formas verbales se pueden agrupar en dos grandes clases según que presenten o no variantes de persona, número, tiempo y modo:

1) formas personales: son las que expresan las personas gramaticales

indicativo: narra los hechos de la realidad

subjuntivo: considera la acción sin que se afirme con seguridad su realización 
potencial o condicional: dice que el hecho sólo ocurrirá si se cumple una condición

imperativo: ordena que se ejecute una acción

2) formas no personales: no indican modo, tiempo ni persona

infinitivo: que equivale al sustantivo del verbo

gerundio: es como un adverbio verbal

participio: se considera como un adjetivo verbal

Los verbos también se pueden clasificar según posean o no estructura argumental en:

Principales: son los únicos que pueden ser predicados y por lo tanto que pueden constituir por sí solos una oración.

Auxiliares: participan en la predicación como elementos de apoyo de un verbo principal a través de los cuales se expresan las nociones relacionadas con el tiempo, el modo, el aspecto, la persona y el número. Los verbos auxiliares por antonomasia en español son: haber, ser y estar. Además de éstos, existen en castellano otros verbos que pueden actuar como auxiliares: ir a, acabar de, tener que, deber, poder, etc. que necesariamente completan su semántica con otras palabras. Algunos ejemplos son: puede realizar, debe ser escrito, se debe registrar, trata de comprender, ayuda a entender, lleva a cabo, pasa a ser, etc.

Por lo tanto ambos verbos (auxiliar y principal) componen un complejo verbal que funciona como una unidad y está compuesto por un verbo en forma personal: el auxiliar $y$ un verbo en forma no personal (infinitivo, gerundio o participio): el principal [Lagunilla '95].

$\checkmark$ Adverbio: complementa, modifica, define y precisa el significado del verbo, del adjetivo, de otro adverbio o de una oración completa. Semánticamente, suele expresar ideas de locación, temporalidad, modo, etc.

Ejemplo: acerca, como, otros, etc.

$\checkmark$ Palabras coordinativas:

preposición: introduce un modificador articulado como complemento, es decir, formado por subordinante (preposición) + término. Las preposiciones pueden subordinar sustantivos, adjetivos y adverbios a otros sustantivos, adjetivos 0 adverbios a verbos e incluso a otra clase de palabras.

Las preposiciones castellanas son: $a$, ante, bajo, cabe, con, contra, de, desde, en, entre, hacia, hasta, para, por, según, sin, so, sobre, tras [Enciclopedia '90].

conjunción: coordina o une palabras, construcciones y palabras, cuando dichos términos son de la misma función sintáctica.

Algunos de ellos son: $y, e, n i, 0, u, y a$, pero, mas, sino, sin embargo, no obstante, porque, pues, como, que, luego, asi que, cuando, por tanto, con que, etc [Enciclopedia '90].

Las palabras en general, pueden dividirse en dos grandes grupos: 
$>$ Categorías léxicas: hacen referencia al mundo, son descriptivas, evocan imágenes, determinan criterios de adecuación referencial en su uso o criterios de verdad. Son denotativos.

> Categorías funcionales: expresan nociones gramaticales tales como tiempo, persona, modo. Son ejemplos de esta categoría: preposiciones, verbos auxiliares, conjunciones, verbos con significado funcional, todos ellos se utilizan para dar forma y articulación a una frase. Un ejemplo de verbo funcional es ser [Lagunilla '95].

Para determinar a qué grupo pertenece cada palabra, se realizan dos operaciones de comprobación:

> Eliminación: si se puede prescindir de ellas y no por eso pierde sentido o significado la cláusula. Ejemplo: "Es un noble caballero que llegó a conocer todo lo relacionado con los secretos del Rey", esta frase se puede recrear eliminando palabras como: es, un, todo, 10, relacionado... "Noble caballero que llegó a conocer los secretos del Rey", se concluye entonces que estas palabras eliminadas son funcionales.

> Reemplazo: si se pueden reemplazar por palabras de significación funcional y no se altera el sentido de la frase. Ejemplo: "Consideraciones sobre la definición de palabra dentro del contexto de este trabajo" puede expresarse como "Consideraciones sobre la definición de palabra en este trabajo" entonces "dentro del contexto de" forman una unidad funcional. 


\section{APENDICE B}

\section{REGLAS DE NORMALIZACION}

\section{Regla 1}

Alcance [artículos y palabras coordinativas (preposiciones y conjunciones)]

Acción [no se cuentan]

Razón [no tienen peso a la hora de definir un concepto]

Excepción [cuando los artículos en una oración cumplen la función de un sustantivo se cuentan]

\section{Regla 2}

Alcance [pronombres]

Acción [no se cuentan]

Razón [son palabras no descriptivas]

Excepción [ los pronombres demostrativos esto, éste, aquél y sus formas masculina, femenina y los correspondientes plurales cuando llevan acento están usados como sustantivos o adjetivos]

\section{Regla 3}

Alcance[formas personales y reflexivas]

Acción [se cuentan como una sola palabra]

Razón[tienen peso semántico sólo si se los considera en conjunto]

Ejemplo [ se convierte, ha guardado, fue comprado, hizo uso]

\section{Regla 4}

Alcance [verbos vacíos o livianos (que necesitan de otras palabras para definir su semántica)]

Acción [se cuentan como una sola palabra]

Razón [tienen peso semántico sólo si se los considera en conjunto]

Ejemplo [tuvo sueño, dio consuelo, se puede realizar, debe llenar, trata de comprender, pasa a ser, lleva a cabo]

$\underline{\text { Regla } 5}$

Alcance [verbo sery estar (cuando se puede reemplazar por ser)]

Acción [no se cuentan]

Razón [son prescindibles en una oración que expresa una definición, son verbos funcionales]

\section{$\underline{\text { Regla } 6}$}

Alcance [conjunto de palabras que forman un símbolo del LEL]

Acción [se cuentan como una sola palabra]

Razón [expresan un concepto] 
Ejemplo [Planilla de ocupación de habitaciones, fichero de pasajeros, pasajero / huésped / pax]

\section{Regla 7}

Alcance [conjunto de palabras que designan un elemento del UdD o una frase de uso común]

Acción [se cuentan como una sola palabra]

Razón [expresan un concepto]

Ejemplo [control remoto TV, servicios turísticos, punto de vista, tarjeta magnética, teniendo en cuenta]

Regla 8

Alcance [caracteres especiales y números]

Acción [se cuentan como una sola palabra]

Razón [se consideran en conjunto con los otros caracteres que acompañan pues describen un único objeto]

Ejemplo [4598, \$135, malenac@gmx.net, \#25]

\section{Regla 9}

Alcance [abreviaturas que acompañan a otras palabras]

Acción [se cuentan como una sola palabra]

Razón [expresan un concepto]

Ejemplo [10 km, 22:30 hrs., Sr. Ramírez]

\section{Regla 10}

Alcance [frases y estructuras]

Acción [se cuenta la estructura completa como una palabra]

Razón [existen indicadores individuales que se repiten a lo largo del texto [Wilson '97]]

Ejemplo [estructura si... entonces] 


\section{DEFINICIONES DE LAS MEDICIONES PROPUESTAS}

\section{DEFINICIONES PARA EL SÍMBOLO DEL LEL}

Definición 1: dada una entrada $x$ de un LEL, se indica con CP(x) la cantidad de palabras que intervienen en su definición, incluyendo todas las ocurrencias de una misma palabra.

Definición 2: dada una entrada $x$ de un LEL, se indica con $C O(x)$ la cantidad de oraciones que se usaron para definir el símbolo $x$.

Definición 3: una palabra p perteneciente a una entrada $x$ de un LEL, se denominará palabra normalizada si luego de aplicarle las reglas de normalización, no ha sido eliminada.

Definición 4: dada una entrada $x$ de un $L E L, x^{*}$ representa el vector de dimensión $C P(x)$ que tiene un 1 en cada posición y se indicará como $R_{k}\left(x^{*}\right)$ al vector resultante de aplicar la regla k-ésima al vector $x^{*}$ que transforma 1 en O si la palabra no es normalizada.

Definición 5: dada una entrada $x$ de un LEL se indica con CPN $(x)$ la cantidad de palabras normalizadas que son las que cumplen con la Definición 3 y que intervienen en su definición.

Definición 6: dada una entrada $x$ de un LEL, se indica con CS(x) la cantidad de símbolos del LEL que intervienen en su definición.

Definición 7: dada una entrada $x$ de un LEL, se indica con CVN $(x)$ la cantidad de verbos normalizados que intervienen en la definición de la entrada $y$ que cumplen con la Definición 3.

Definición 8: dada una entrada $x$ de un LEL, se indica con COPN(x) la cantidad de otras palabras normalizadas que no son símbolos del LEL ni verbos normalizados.

Definición 9: dada una entrada $x$ de un LEL se indica con $\operatorname{COER}(x)$ al coeficiente de redundancia que es el cociente entre la cantidad de palabras normalizadas y la cantidad de palabras utilizadas en la definición de un símbolo $\times$ del LEL.

Definición 10: dada una entrada $x$ de un LEL se indica con COES $(x)$ al coeficiente de simplicidad que es el cociente entre la cantidad de verbos 
normalizadas y la cantidad de oraciones utilizadas en la definición de un símbolo $\times$ del LEL.

Definición 11: dada una entrada $x$ de un LEL se indica con FI(x) y se denomina Fan In a la cantidad de todos los símbolos que se usan para definir el símbolo $x$.

Definición 12: dada una entrada $x$ de un LEL se indica con $F O(x)$ y se denomina Fan Out a la cantidad de veces que el símbolo x es usado en la definición de otros símbolos.

Definición 13: dada una entrada $x$ de un LEL se denomina FOI(x) y se calcula como la razón entre el Fan Out y el Fan In para el símbolo $x$ del LEL a la relación entre la cantidad de veces que un símbolo $x$ es usado en las definiciones de otros símbolos y la cantidad de símbolos que usa en su propia definición.

Definición 14: dado un LEL Ly una entrada $x$ se indica con TU(x) la tasa de uso de la entrada $x$ y se calcula como la relación entre el Fan Out de la entrada $x$ y la cantidad de entradas que se definieron para el LEL L.

\section{DEFINICIONES PARA EL LEL}

Definición 1: dado un LEL L se indica con $\operatorname{CSL}(L)$ la cantidad de símbolos que se definieron.

Definición 2: dado un LEL $L$ se indica con $D S L C\left(L, C_{k}\right)$ la cantidad de símbolos en cada grupo $C_{k}$ de una clasificación dada.

Definición 3: dado un LEL L se indica con CPL(L) la cantidad de palabras del LEL L que se usan para definir todas las entradas.

Definición 4: dado un LEL $L$ se indica con CPNL(L) la cantidad de palabras normalizadas del LEL L que se usan para definir todas las entradas.

Definición 5: dado un LEL L se indica con CVNL(L) la cantidad de verbos normalizadas del LEL L que usa para expresar todas las entradas.

Definición 6: dado un LEL L se indica con CSUL(L) la cantidad de símbolos usados en el LEL L para definir todas las entradas.

Definición 7: dado un LEL $L$ se indica con COPNL(L) la cantidad de otras palabras normalizadas del LEL L que se usan para definir todas las entradas. 
Se puede afirmar que la estadística es la ciencia del análisis e interpretación de los datos. Involucra la recolección, clasificación, síntesis y organización de las observaciones hechas para una clase de elementos de la que se puede medir sólo una parte o muestra, considerada representativa del total, o todos los elementos, en cuyo caso se evaluará la población.

\section{Mediciones De LA Tendencia Central}

La mayor parte de las series de datos muestran una clara tendencia a agruparse alrededor de un cierto punto central o valor promedio. Este valor descriptivo típico es una medición de tendencia central o ubicación.

\subsection{MEDIA ARITMÉTICA}

Es una medida de la tendencia central de un conjunto de datos. La fórmula usada es:

$$
\bar{x}=\frac{\sum_{i=1}^{N} x_{i}}{N}
$$

siendo: $x_{i}=$ iésima observación de la variable $x$

$N=$ número de elementos de la población

Ventajas:

a) es un concepto intuitivamente claro

b) es única para cada conjunto de datos

c) es útil para llevar a cabo procedimientos estadísticos, como la comparación de medias de varios conjuntos de datos

Desventajas:

a) puede verse afectada por valores extremos que no son representativos del resto de los datos

b) puede mal interpretarse si los datos no entran en un grupo homogéneo

\subsection{MEDIANA}

Es un solo valor calculado a partir del conjunto de datos que mide la observación central de éstos. La mitad de los elementos está por encima de ella y el resto estará por debajo.

Para calcular la mediana, los elementos deberán estar ordenados en forma creciente o decreciente. Si hay un número par de observaciones, la mediana es el 
promedio de los dos elementos centrales; si es impar, la mediana será el valor central y para encontrarlo se usa la fórmula del punto de posicionamiento:

$$
\frac{N+1}{2}
$$

Al calcular la mediana se ignora el hecho de que pueda haber valores de observaciones repetidos.

Ventajas:

a) los valores extremos no afectan el valor de la mediana, pero sí la cantidad de observaciones

b) es fácil de entender y se puede calcular a partir de cualquier tipo de datos, inclusive no numéricos

c) la mediana es mucho mejor que la media aritmética para eliminar el efecto de uno o dos elementos "externos" : puntos de datos extremos

Desventajas:

a) debido a que es una posición promedio, los datos deben ser previamente ordenados, lo que resulta ser consumidor de tiempo, sobre todo si se trata de una población grande

\subsection{MODA}

Es una medida de la tendencia central diferente de la media pero un tanto parecida a la mediana, pues no se calcula mediante algún proceso aritmético. La moda es aquél valor que más se repite en el conjunto de datos.

Ventajas:

a) no se ve mayormente afectada por valores extremos

b) sirve tanto para valores cuantitativos como cualitativos

c) se puede aplicar sin importar que tan grandes o pequeños sean los valores de la población e independientemente de cuál sea su dispersión

Desventajas:

a) puede suceder que un conjunto de datos no tenga un valor modal pues no contiene valores que se presenten más de una vez

b) otras veces cada valor es la moda pues se presenta el mismo número de veces

c) también sucede que un conjunto presenta dos, tres o más valores modales, entonces es difícil interpretarlos

\subsection{CUARTILES}

Estas mediciones son descriptivas y dividen los datos ordenados en cuatro cuartos.

$\checkmark$ Cuartil inferior $\rightarrow Q_{i}$ es la mediana de los valores que son menores que la mediana de las observaciones

$\checkmark$ Mediana $\rightarrow Q_{m}$ es el valor para el cual el $50 \%$ de las observaciones son menores y el $50 \%$ de las observaciones son mayores 
$\checkmark$ Cuartil superior $\rightarrow Q_{s}$ es la mediana de los valores que son mayores que la mediana de las observaciones

Se usa para zanjar los potenciales problemas introducidos por los valores extremos de los datos.

Para determinar los valores extremos, se definen algunas medidas:

$\checkmark$ Longitud de la caja: es la distancia entre el cuartil superior y el inferior

$$
L=Q_{s}-Q_{i}
$$

$\checkmark$ Extremos de la distribución: estos puntos representan los límites teóricos entre los cuales se espera encontrar todos los datos si la distribución fuera normal.

$$
\begin{aligned}
& \text { Extremo superior }=Q_{s}+1.5 \mathrm{~L} \\
& \text { Extremo inferior }=Q_{s}-1.5 \mathrm{~L}
\end{aligned}
$$

$\checkmark$ Valores fuera de la caja: representan los valores extremos, son puntos que de alguna manera son excepcionales. Sirven para identificar comportamientos anormales.

\section{MEDICIONES DE LA DISTRIBUCióN}

En el análisis estadístico no basta el cálculo e interpretación de las medidas de tendencia central o de posición, ya que, por ejemplo, si se pretende describir un conjunto de datos con la media aritmética, no se logra reflejar la realidad, pues suelen existir datos extremos inferiores y superiores a la media aritmética que no están siendo bien representados por este parámetro.

Existen algunas mediciones útiles de ubicación "no central". La variación es la cantidad de dispersión o propagación en los datos. Dos series de datos pueden tener la misma tendencia central pero diferir enormemente en términos de la variación.

La dispersión de la distribución es una característica importante para entender y medir porque proporciona información adicional para juzgar la confiabilidad de la medida de tendencia central. Si los datos se encuentran ampliamente dispersos, la posición central es menos representativa de los datos como un todo.

Las mediciones de este tipo más ampliamente usadas toman en cuenta cómo se distribuyen los valores alrededor de la media, ellas son la varianza y su raíz cuadrada, el desvío estándar y el coeficiente de variación.

\subsection{DESVÍO ESTÁNDAR}

Da idea del error que se comete al usar el valor medio de la población como característico de cualquier individuo. Es una medida más "entendible" que la varianza pues está en las mismas unidades que los datos que se están evaluando.

$$
S=\sqrt{\frac{N \sum_{i=1}^{N} x_{i}^{2}-\left(\sum_{i=1}^{N} x_{i}\right)^{2}}{N^{2}}}
$$


siendo: $x_{i}=$ i-ésima observación de la variable $x$

$N=$ número de elementos de la población

\subsection{COEFICIENTE DE VARIACIÓN}

La desviación estándar no puede ser la única base para la comparación de dos distribuciones. Por ejemplo, si se tiene una población con una desviación estándar de 10 y una media de 5 el grado de dispersión de los datos no será el mismo que para una población que también tiene una desviación estándar de 10 pero una media de 5000 ya que en este caso la variación con respecto a la media es insignificante.

El coeficiente de variación tiene en cuenta el valor del desvío estándar y la media aritmética, para establecer un número relativo, que hace comparable el grado de dispersión entre dos o mas poblaciones. Se define como:

$$
C V=\frac{S}{\bar{x}}
$$

siendo: $S$ = el desvío estándar

$\bar{x}=$ media aritmética

\subsection{TEOREMA DE CHEBysheV}

La desviación estándar permite determinar, con un buen grado de precisión, dónde están localizados los valores de una distribución con relación a la media de la población. Esto se puede realizar gracias al teorema del matemático ruso P. L. Chebyshev (1821-1894) que dice que independientemente de la forma de una distribución, al menos el $75 \%$ de los valores estarán en el intervalo que queda definido por \pm 2 desviaciones estándar a partir de la media de la distribución y al menos el $89 \%$ de los valores estarán en el intervalo que queda definido por \pm 3 desviaciones estándar a partir de la media de la distribución.

\subsection{DISTRIBUCIÓN NORMAL}

Se denomina así a la distribución de una variable aleatoria continua que tiene una curva de un solo pico y con forma de campana. La media cae en el centro de la distribución y la curva es simétrica con respecto a una línea vertical que pasa por la media. Los dos extremos se extienden indefinidamente sin tocar nunca el eje horizontal.

Existen dos razone básicas por las cuales la distribución normal o gaussiana ocupa un lugar prominente en la estadística. Primero porque tiene alguna propiedades que la hacen aplicable a un gran número de situaciones en las que es necesario hacer inferencias. Segundo, la distribución normal casi se ajusta a las distribuciones de frecuencias reales observadas en muchos fenómenos incluyendo, por ejemplo, características físicas, resultados de procesos físicos y mediciones de interés para administradores de proyectos.

La distribución normal de probabilidad tiene como característica que la media, la mediana y la moda coinciden. 
Para definir una distribución normal, se necesitan dos parámetros: la media 0 promedio y el desvío estándar. Cualesquiera sean los valores de estos parámetros, el área total bajo la curva es 1 , entonces, matemáticamente se cumple que:

1- aproximadamente el $68 \%$ de todos los valores se encuentra dentro de \pm 1 desvío estándar de la media.

2- aproximadamente el $95.5 \%$ de todos los valores se encuentra dentro de \pm 2 desvíos estándar de la media.

3- aproximadamente el $99.7 \%$ de todos los valores se encuentra dentro de \pm 3 desvíos estándar de la media.

\section{REGRESIÓN SIMPLE Y CORRELACIÓN}

La regresión y los análisis de correlación muestran cómo determinar tanto la naturaleza como la fuerza de una relación entre dos variables. En el análisis de regresión se desarrollan ecuaciones de estimación para relacionar las variables conocidas con la desconocida, teniendo el patrón de esa relación se aplica el análisis de correlación para determinar el grado en el que están relacionadas las variables. La variable conocida se llama independiente y la desconocida, dependiente. Es importante considerar las relaciones encontradas por la regresión como relaciones de asociación pero no necesariamente de causa y efecto, a menos que se tenga razones específicas para creer que los valores de la variable dependiente son ocasionados por los valores de la variable independiente.

\subsection{DIAGRAMAS DE DISPERSIÓN}

Para saber si existe o no relación entre dos variables, lo primero que se debe hacer es graficar los datos observados, este esquema se conoce como diagrama de dispersión. A partir de él se puede, visualmente, buscar patrones que indiquen si las variables están relacionadas o son simples observaciones azarosas. Es común intentar trazar líneas de forma tal que un número igual de puntos caiga a cada lado de la línea.

\subsection{LÍNEA DE REGRESIÓN}

Si es posible trazar una línea entre los puntos, significa que es posible establecer una ecuación matemática que represente esa línea.

$$
y=a+b x
$$

donde: $a$ intersección de la línea con el eje de ordenadas ( $Y$ )

$b=$ pendiente de la recta

$x=$ variable independiente

$y=$ variable dependiente

de forma tal que dado un valor cualquiera de $x$ se puede calcular el valor de $y$.

Una vez determinada una línea de regresión se usará la notación

$$
\hat{y}=a+b x
$$

para simbolizar los puntos estimados, es decir, los que caen en la línea de estimación. 


\subsection{ANÁLISIS DE CORRELACIÓN}

Indica el grado hasta el cual una variable está linealmente relacionada con otra. Una de las medidas usadas para describir la correlación entre dos variables es el coeficiente de determinación: $r^{2}$. Este coeficiente se desarrolla a partir de la relación entre dos tipos de variación de los valores de y en un conjunto de datos alrededor de:

1- la línea de regresión

2- su propia media

$$
r^{2}=1-\frac{\sum(y-\hat{y})^{2}}{\sum(y-\bar{y})^{2}}
$$

Si la relación es lineal perfecta, el coeficiente de determinación será 1. Cuando no es posible explicar la variación de la variable dependiente en función de la variable independiente entonces el coeficiente de determinación será 0 .

La relación entre el coeficiente de correlación y el coeficiente de determinación está dada por:

\section{Coeficiente de correlación $=\sqrt[2]{\text { Coeficiente de determinación }}$}

Mientras que el coeficiente de determinación permite puede explicar la variación, el de correlación indica el grado de asociación.

Otra interpretación del coeficiente de determinación dice que es la fracción de la variación total de $y$ que es explicada por la línea de regresión, dicho de otra manera mide qué tan bien $x$ explica $y$, esto es, el grado de asociación entre $x$ e $y$.

Hay dos precauciones a tener en cuenta:

1. la existencia de una relación no indica causa-efecto

2. el poder asegurar que las series no son independientes, no quiere decir que la relación sea tan estrecha como para estimar valores de la variable dependiente conociendo la variable independiente, esto depende del error de estimación aceptado.

Nota: para la elaboración de este capítulo se usaron las siguientes referencias bibliográficas:

[Guarín '02]; [Excel '00]; [Berenson '96]; [Levin '96] 


\section{APENDICE E}

\section{LEL "HOTEL"}

\section{Administración}

- Notion

- Es un departamento del Hotel.

- Administra todas las actividades relacionadas con el manejo económico del Hotel.

- Behavioral responses

- Atiende todas las cuestiones relacionadas al personal.

- Hace la facturación para el pasajero / huésped / pax.

\section{agencia / agencia de viajes}

- Notion

- Es una organización externa e independiente del Hotel.

- Administra las ventas de servicios turísticos al pasajero / huésped / pax.

- Behavioral responses

- Puede hacer una solicitud de reserva o una cancelación de reserva para el pasajero / huésped / pax.

- Puede hacer un voucher para el pasajero / huésped / pax.

aviso de mensaje

- Notion

- Contiene el registro de los mensajes recibidos cuando el pasajero / huésped / pax no se halla en el Hotel, junto con el número de habitación.

- Lo registra la recepción.

- Se almacena temporariamente en la recepción.

- Behavioral responses

- Lo recibe el pasajero / huésped / pax.

\section{cancelación de reserva}

\section{- Notion}

- Es la baja de la solicitud de reserva de un pasajero / huésped / pax.

- La hace el pasajero / huésped / pax, una agencia u otro hotel vía telefónica, fax, email o personalmente.

- Se hace en la recepción.

- Behavioral responses

- Elimina la solicitud de reserva del pasajero / huésped / pax de la planilla de reservas.

- Actualiza la disponibilidad de habitaciones en la planilla de ocupación de habitaciones. 
- Contiene: comprobante de consumos de cafetería y/o restaurant, comprobante de gastos de lavandería, comprobante de consumos del minibar, comprobante de llamados telefónicos y voucher, si correspondiere.

- Se guarda en un mueble de archivo para carpetas en la recepción.

- Behavioral responses

- La administración la utiliza para hacer la facturación.

check in

- Notion

- Es el conjunto de los trámites necesarios para el ingreso de un pasajero / huésped / pax al Hotel.

- Lo atiende el recepcionista.

- Se hace en la recepción.

- Behavioral responses

- Si el pasajero / huésped / pax presenta un voucher entonces el recepcionista lo almacena en la carpeta de la habitación.

- Se busca el nombre del pasajero / huésped / pax en la planilla de reservas.

- Si el pasajero / huésped / pax se presenta por primera vez al Hotel debe completar y firmar la planilla del pasajero sino se buscan sus datos personales por nombre en el fichero de pasajeros.

- El recepcionista controla la planilla del pasajero y actualiza el fichero de pasajeros.

- Se asigna un número de habitación al pasajero / huésped / pax.

- Al actualizar la disponibilidad de habitaciones, se actualiza la planilla de ocupación de habitaciones.

- Se agrega el número de habitación asignado en la planilla de reservas.

- Se hace una tarjeta codificada para el número de habitación.

- Se entrega la tarjeta codificada y el control remoto TV.

- Si el pasajero / huésped / pax lo solicita se le provee la llave de la caja de seguridad de la habitación para guardar objetos de valor y/o dinero.

- Si el pasajero/huésped / pax lo solicita se le entrega la llave del minibar / frigobar.

check out

- Notion

- Es el conjunto de los trámites necesarios para el egreso del pasajero / huésped / pax del Hotel.

- Lo atiende el recepcionista.

- Se hace en la recepción.

- Behavioral responses

- Se provee al pasajero / huésped / pax junto con la factura: comprobante de gastos de lavandería, comprobante de consumos de cafetería y/o restaurant, comprobante de consumos del minibar y comprobante de llamados telefónicos.

- Se recibe el pago del pasajero / huésped / pax.

- Se agrega el sello de Pagado en la factura

- Se envía la copia de la factura del pasajero / huésped / pax al Departamento administración.

- Se recibe la tarjeta codificada, el control remoto TV, la llave del minibar / frigobar si estuviera en poder del pasajero / huésped / pax y la llave de la caja de seguridad si estuviera en poder del pasajero / huésped / pax 
- Al actualizar la disponibilidad de habitaciones, se actualiza la planilla de ocupación de habitaciones.

\section{comprobante de consumos de cafetería y/o restaurant}

- Notion

- Contiene el número de habitación, el detalle y el importe de los consumos hechos y la firma del pasajero / huésped / pax o acompañantes según corresponda.

- Lo provee el Departamento gastronomía.

- Se almacena en la carpeta de la habitación.

- Behavioral responses

- Lo hace el Departamento gastronomía.

- El pasajero / huésped / pax lo firma.

- El Departamento gastronomía lo envía a la recepción.

\section{comprobante de consumos del minibar}

- Notion

- Contiene el número de habitación, el importe y el detalle de los artículos del minibar / frigobar consumidos por el pasajero / huésped / pax.

- Lo provee el Departamento mucamas.

- Se almacena en la carpeta de la habitación.

- Behavioral responses

- Lo hace diariamente el Departamento mucamas.

- El Departamento mucamas lo envía a la recepción.

\section{comprobante de gastos de lavandería}

- Notion

- Contiene el importe del trabajo de lavandería solicitado por el pasajero / huésped / paxy su número de habitación.

- Lo provee el Departamento mucamas.

- Se almacena en la carpeta de la habitación.

- Behavioral responses

- Lo hace el Departamento mucamas.

- El Departamento mucamas lo envía a la recepción.

\section{comprobante de llamados telefónicos}

- Notion

- Contiene el importe de la llamada telefónica y el número de habitación del pasajero / huésped / pax.

- Lo provee el sistema telefónico.

- Se almacena en la carpeta de la habitación.

- Behavioral responses

- El sistema telefónico lo hace en forma automática.

\section{disponibilidad de habitaciones}

- Notion

- Contiene la cantidad y tipo de habitación desocupada. 
- Se obtiene de la planilla de ocupación de habitaciones como diferencia entre la capacidad total del Hotel y la suma de las habitaciones ocupadas y reservadas.

- Behavioral responses

- Se actualiza cuando se actualiza la planilla de ocupación de habitaciones.

día de egreso

- Notion

- Contiene la fecha en que el pasajero / huésped / pax deja el Hotel.

- La provee el pasajero / huésped / pax o la obtiene el recepcionista.

- Se almacena en la planilla de reservas.

- Behavioral responses

- La diferencia entre esta fecha y el día de ingreso permite calcular los días que el pasajero / huésped / pax ha permanecido en el Hotel.

- La administración la utiliza para el cálculo de la facturación.

día de ingreso

- Notion

- Contiene la fecha en que el pasajero / huésped / pax ingresa al Hotel.

- La provee el pasajero / huésped / pax o la obtiene el recepcionista.

- Se almacena en la planilla de reservas.

- Behavioral responses

- La diferencia entre el día de egreso y ésta permite calcular los días que el pasajero / huésped / pax ha permanecido en el Hotel.

- La administración la utiliza para el cálculo de la facturación.

\section{$\underline{\text { facturación }}$}

- Notion

- Es el proceso de emisión de la factura del pasajero / huésped / pax.

- Se hace en la administración.

- Behavioral responses

- Se busca el nombre del pasajero / huésped / pax en la planilla de reservas y se obtiene el número de habitación, el día de ingreso y el día de egreso.

- Se envía a la administración la carpeta de la habitación correspondiente al número de habitación del pasajero / huésped / pax, junto con el día de ingreso y el día de egreso.

- Se recibe una factura original y una copia desde la administración.

\section{fichero de pasajeros}

- Notion

- Contiene el conjunto de planilla del pasajero.

- Se guarda en un mueble de archivo en la recepción.

- Behavioral responses

- Se utiliza como archivo histórico para el check in a un pasajero / huésped / pax.

Gastronomía

- Notion 
- Es un departamento del Hotel.

- Brinda el servicio de desayuno incluido en la tarifa, el servicio de Restaurant y Cafetería y el servicio de cafetería en la habitación.

- Behavioral responses

- Envía el comprobante de consumos de cafetería y/o restaurant del pasajero/ huésped / pax a la recepción.

\section{habitación}

- Notion

- Es un cuarto del Hotel.

- Puede ser de tipo estándar, suite junior o suite presidencial, todas ellas en la modalidad single/doble.

- Tiene una tarifa determinada y un número que la identifica.

- Aloja al pasajero / huésped / pax.

- Behavioral responses

- Se reserva al hacer una solicitud de reserva.

- Se asigna en el check in.

- Se desocupa en el check out.

\section{lista de precios}

- Notion

- Es un listado que provee el Departamento gerencia.

- Se utiliza para almacenar la tarifa según el tipo y modalidad de la habitación.

- Está en la recepción y en la administración.

- Behavioral responses

- La utiliza la administración para obtener la tarifa de una habitación.

- La utiliza la recepción para informar la tarifa de una habitación cuando se efectúa una solicitud de reserva o pedido de alojamiento

\section{Mantenimiento}

- Notion

- Es un departamento del Hotel.

- Hace las tareas de conservación y reparaciones programadas y solicitadas desde otros departamentos.

- Behavioral responses

- Hace la tarea requerida en una habitación.

\section{minibar / frigobar}

\section{- Notion}

- Es una pequeña heladera que tiene una llave.

- Se utiliza para conservar bebidas y alimentos refrigerados.

- Está en cada habitación.

- Behavioral responses

- El pasajero / huésped / pax puede consumir los elementos que contiene.

- Diariamente el Departamento mucamas controla su contenido y repone los elementos faltantes. 
Mucamas

- Notion

- Es un departamento del hotel dirigido por la Gobernanta.

- Atiende el servicio de lavandería de todo el Hotel, incluyendo los pedidos particulares del pasajero / huésped / pax .

- Hace la limpieza de cada habitación.

- Hace la limpieza de los sectores públicos.

- Atiende el pedido de limpieza extra enviado por la recepción.

- Registra los consumos del minibar / frigobar.

- Behavioral responses

- Maneja la tarjeta codificada maestra.

- Envía a la recepción comprobante de gastos de lavandería y el comprobante de consumos del minibar del pasajero / huésped / pax.

no show

- Notion

- Es la baja de la solicitud de reserva por no presentación del pasajero / huésped / pax.

- Lo hace el recepcionista.

- Se hace en la recepción.

- Behavioral responses

- Si el pasajero / huésped / pax no se presenta el día de ingreso hasta las 06 hs. del día siguiente entonces se elimina la solicitud de reserva en la planilla de reservas.

- Se actualiza la disponibilidad de habitaciones en la planilla de ocupación de habitaciones

pago

- Notion

- Es el proceso para cancelar el importe de la facturación al pasajero / huésped / pax.

- Lo hace el pasajero / huésped / pax.

- Se hace en la recepción.

- Behavioral responses

- Si es en efectivo o cheque se guarda en la caja de la recepción.

- Si es con tarjeta de crédito se solicita la autorización, se hace el cupón, el pasajero I huésped / pax lo firma y se guarda en la recepción.

- Si se debita de una cuenta corporativa el pasajero / huésped / pax firma la factura y se envía a la administración.

pasajero / huésped / pax

- Notion

- Es una persona que tiene una solicitud de reserva o hace un pedido de alojamiento

- Behavioral responses

- Provee los datos requeridos por el recepcionista.

- Puede presentar un voucher.

- Completa la planilla del pasajero la primera vez que se presenta en el Hotel.

- Recibe la tarjeta codificada y el control remoto TV. 
- Puede solicitar la llave de la caja de seguridad y/o minibar / frigobar.

- Puede hacer consumos en Cafetería y/o Restaurant, consumos del minibar / frigobar, llamados telefónicos y gastos de lavandería.

- Puede hacer un pedido del servicio de despertador.

- Puede hacer un pedido de limpieza extra y/o un pedido de mantenimiento extra.

- Puede recibir un aviso de mensaje.

- Recibe la factura y los comprobantes de gastos.

- Hace el pago.

- Devuelve la tarjeta codificada, el control remoto TV y la llave del minibar / frigobar y/o de la caja de seguridad de la habitación si las solicitó.

- Puede hacer una cancelación de reserva.

pedido de alojamiento

- Notion

- Es el pedido de una habitación hecho en forma personal, sin una solicitud de reserva.

- Lo hace una persona.

- Se recibe en la recepción.

- Behavioral responses

- Se consulta la planilla de ocupación de habitaciones.

- Si hay disponibilidad de habitaciones se registran sus datos personales en la planilla de reservas.

- Se hace el check in.

pedido de limpieza extra

- Notion

- Es la solicitud de un servicio de limpieza adicional.

- Lo hace el pasajero / huésped / pax.

- Se recibe en la recepción.

- Behavioral responses

- Se registra el número de habitación del pasajero / huésped / pax que solicitó el servicio.

- Se hace la lista con los pedidos recibidos.

- Se envía la lista al Departamento mucamas.

pedido de mantenimiento extra

- Notion

- Es la solicitud de un servicio de reparaciones.

- Lo hace el pasajero / huésped / pax.

- Se recibe en la recepción.

- Behavioral responses

- Se registra el número de habitación del pasajero / huésped / pax que solicitó el servicio y el tipo de reparación a efectuar.

- Se hace la lista con los pedidos de reparaciones.

- Se envía el pedido al Departamento mantenimiento. 
- Notion

- Es la solicitud del servicio que brinda el Hotel para despertar al pasajero / huésped $\angle$ pax en un horario determinado.

- Lo hace el pasajero / huésped / pax.

- Se atiende en la recepción

- Behavioral responses

- Se registra el número de habitación y la hora solicitada.

- Llegada esa hora, se llama por teléfono al número de habitación del pasajero/ huésped / pax.

planilla de ocupación de habitaciones

- Notion

- Contiene el estado de ocupación según el tipo y modalidad de la habitación.

- Se guarda en la recepción.

- Behavioral responses

- Se actualiza cuando se hace una solicitud de reserva, una cancelación de reserva, un no show, un check in o un check out.

planilla de reservas

- Notion

- Contiene la solicitud de reserva de un pasajero / huésped / pax.

- Se hace una por día y se conserva en una carpeta en la recepción hasta el check out de todos los pasajero / huésped / pax que figuran en ella, luego se guardan en un archivo.

- Behavioral responses

- Se utiliza para actualizar la planilla de ocupación de habitaciones.

- En el check in se le agrega el número de habitación asignado al pasajero / huésped / pax.

- Se actualiza cuando se hace una solicitud de solicitud de reserva, cancelación de reserva, no show o pedido de alojamiento del pasajero / huésped / pax.

planilla del pasajero

- Notion

- Contiene el nombre, número de documento, otros datos personales y comerciales, firma, día de ingreso, procedencia del pasajero / huésped / pax y datos del vehículo, si correspondiere.

- La completa el pasajero / huésped / pax en el check in.

- Se almacena en el fichero de pasajeros en la recepción del Hotel..

- Behavioral responses

- La completa el pasajero / huésped / pax la primera vez que se presenta en el Hotel.

- Se utiliza para actualizar el fichero de pasajeros.

recepcionista

- Notion

- Persona que atiende el Departamento recepción del Hotel.

- Behavioral responses

- Recibe una caja con dinero cuando se hace cargo de la recepción. 
- Atiende una solicitud de reserva o un pedido de alojamiento.

- Controla la disponibilidad de habitaciones.

- Hace el check in.

- Recibe y almacena en la carpeta de la habitación: comprobante de consumos de cafetería y/o restaurant, comprobante de gastos de lavandería, comprobante de consumos del minibar, comprobante de llamados telefónicos y el voucher del pasajero / huésped / pax.

- Atiende el pedido del servicio de despertador.

- Recibe los pedido de limpieza extra y pedido de mantenimiento extra del pasajero / huésped / pax.

- Atiende los mensajes y si el pasajero / huésped / pax no está, completa el aviso de mensaje.

- Envía la carpeta de la habitación a la administración para la facturación.

- Hace el check out.

- Ante la cancelación de reserva o el no show actualiza la planilla de reservas.

- Hace el pedido de reposición de insumos.

\section{Recepción}

- Notion

- Es un departamento interrelacionado con todos los departamentos del Hotel.

- Atiende todas las actividades relativas al ingreso, permanencia y egreso del pasajero / huésped / pax.

- Behavioral responses

- Es el lugar de trabajo del recepcionista.

- Almacena toda la información relativa al movimiento del pasajero / huésped / pax.

\section{reposición de insumos}

- Notion

- Es la acción de pedir y recibir los elementos necesarios para el funcionamiento de la recepción.

- Lo hace el recepcionista.

- Se hace en la recepción.

- Behavioral responses

- Se hace una lista de elementos faltantes.

- Se envía la lista al Departamento compras.

- Se recibe el pedido desde el Departamento compras.

- Se firman los formularios de recibido.

\section{solicitud de reserva}

- Notion

- Es un pedido de hospedaje para una persona hecho con anterioridad al día de ingreso.

- Caduca por no show del pasajero / huésped / pax.

- La hace una persona, una agencia u otro hotel vía telefónica, fax, e-mail o personalmente.

- La atiende la recepción.

- Behavioral responses 
- Se consulta en la planilla de ocupación de habitaciones la disponibilidad de habitaciones.

- Se informa la tarifa.

- Se solicita la aprobación a la persona, agencia u otro hotel.

- Se registra en la planilla de reservas el nombre y apellido del pasajero / huésped / pax, tipo y modalidad de la habitación, cantidad de pasajeros, día de ingreso, día de egreso, teléfono, tarifa y observaciones.

- Se actualiza la disponibilidad de habitaciones en la planilla de ocupación de habitaciones.

$\underline{\text { tarifa }}$

- Notion

- Es el costo de la habitación según sus características.

- La provee la lista de precios.

- Se almacena en la lista de precios.

- Behavioral responses

- La utiliza la administración para hacer la facturación.

\section{tarjeta codificada}

- Notion

- Es una tarjeta magnética que tiene grabado un código.

- Según su tipo se utiliza para abrir una o varias habitaciones.

- Está en el Departamento mucamas o en poder del pasajero / huésped / pax.

- Behavioral responses

- Si está destinada al pasajero / huésped / pax tiene un código específico para abrir una determinada habitación.

- Si es para el personal del Departamento mucamas se trata de una tarjeta maestra que permite el acceso a las habitaciones de todo un piso o de todo el Hotel.

voucher

- Notion

- Contiene los datos del pasajero / huésped / pax, el día de ingreso, el día de egreso, la cantidad de pasajeros, nombre y dirección del Hotel.

- Lo provee la agencia.

- Se almacena en la carpeta de la habitación.

- Behavioral responses

- El pasajero / huésped / pax paga el importe del alojamiento en la agencia y éste se acredita en una cuenta corriente del Hotel.

- El recepcionista lo recibe del pasajero / huésped / pax

- Se utiliza para cancelar el importe del ítem correspondiente a alojamiento en la facturación. 